\title{
Design and Validation of a Force-Sensing Piano Key to Assess Pianist Biomechanics
}

\author{
by \\ Francesca Tsimiklis, B.Eng. \\ A Thesis submitted to the \\ Faculty of Graduate and Postdoctoral Affairs \\ in partial fulfillment of the requirements for the degree of
}

Master of Applied Science

Ottawa-Carleton Institute for Mechanical and Aerospace Engineering

Department of Mechanical and Aerospace Engineering

Carleton University

Ottawa, Ontario

June, 2020

(C)Copyright

Francesca Tsimiklis, 2020 


\section{Abstract}

Musicians injuries are surprisingly common. There is limited knowledge behind the biomechanics of pianist performance. This study presents the development of a novel instrumented piano key with integrated strain-based sensors that unobtrusively measures the force applied during finger-key interaction in pianists.

Pianist perception of touch was experimentally assessed to determine sensitivity to changes in key mass properties. In general, pianists were unable to identify significant changes in the mass of the key. The prototype key successfully identified components of contact force and location. Design refinements are presented that will enable a second prototype to completely measure all components of the contact force and its location. The accuracy, precision, and resolution of data collected from this prototype clearly indicate that an instrumented key can be integrated in a piano to assess the finger key interactions in piano performance. 
To Nonna Visentin,

for always reminding me that "every road leads you to Rome" 


\section{Acknowledgements}

I would like to acknowledge those who have assisted me throughout my research.

First and foremost, I would like to thank my supervisor Dr. Donald Russell for his continuous support and patient guidance. Thank you for granting me the opportunity to take part in this exciting research. I am fortunate to have been able to complete my research in such a knowledgable and positive learning environment. Thank you for your encouragement in moments, or emails, where I began to doubt my accomplishments in this research.

I would also like to recognize Dr. Gilles Comeau and the members of the Piano Pedagogy Research Laboratory. Thank you for allowing access to your lab and providing essential insight from a musician's perspective.

To my supportive family; thank you for your ongoing support for me to continue my education. Your patience, guidance, and home-made vegetable lasagnas have continuously driven me to continue in my work. Mom, thank you for always supporting my academic endeavours and being an advocate for women in STEM. From attending science camp to university lectures of women leading in STEM, you have allowed me to be immersed in these dynamic fields, for which I am truly grateful.

Matt, thank you for your endless patience and enthusiasm in all of my research achievements, no matter how small. I would not have been able to complete this research without your assistance whenever I needed a sounding board or a second set

of hands during assembly. You and your family have been a constant cheering squad 
and voice of reason; I am grateful for their assistance while I was away from home. All of my friends who have supported me along this journey, thank you. Your excitement about my research has kept me inspired to continue my work. Jason, thank you for constantly reminding me to take breaks to find a healthy work-life balance; I think by the end I started to listen to you.

I would like to extend my thanks to the Laboratory and Machine Shop Technologists of the Department of Mechanical and Aerospace Engineering for their assistance and wealth of knowledge. Their resources were invaluable in completing my research.

Finally, to everyone who has interacted with this research. Thank you for your enthusiasm in my thesis work and excitement at the potential impact it will have on future musician injury and biomechanics studies. Your interest has provided the motivation to continue research in this evolving field. 


\section{Preface}

This thesis is intended for a multidisciplinary audience, including researchers with an engineering or musical background who may work collaboratively on future research. To ensure the contents of this thesis are also appreciated by non-engineering fields, this thesis will include referencing to common engineering topics, which provide further background on the theory used to develop this thesis. 


\section{Table of Contents}

Abstract $\quad$ iii

Acknowledgments $\quad$ V

Preface $\quad$ vii

Table of Contents viii

List of Tables $\quad$ xiii

List of Figures xiv

Nomenclature $\quad$ xx

1 Introduction 1

1.1 Motivation ............................ 1

1.2 Problem Statement and Contributions . . . . . . . . . . . . 3

1.3 Thesis Outline ....................... 4

2 Review of Literature $\quad 6$

2.1 Piano-User Interaction . . . . . . . . . . . . . . . . . 6

2.2 Musicians and PRMDs . . . . . . . . . . . . . . 10

2.2.1 Importance of Studying the Biomechanics of Finger Contact . 13

2.3 Previous Measurement Techniques . . . . . . . . . . . . . . . . 14 
2.3.1 Pianist Movement Analysis . . . . . . . . . . . . . . . . 14

2.3.2 Pianist Finger-Key Force Measurement . . . . . . . . . . . 17

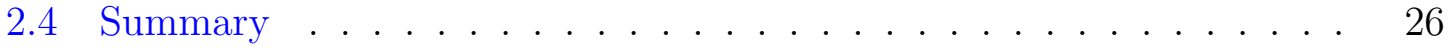

3 Design Approach $\quad 27$

3.1 Evaluation of Potential Design Approaches . . . . . . . . . . . 27

3.1 .1 Biomechanical Approach . . . . . . . . . . . . . . . . . 28

3.1 .2 Optical Measurement Approach . . . . . . . . . . . . . . 30

3.1.3 Acoustic Measurement Approach . . . . . . . . . . . . . . 31

3.1.4 Mechanical Approach . . . . . . . . . . . . . . . . . . . 31

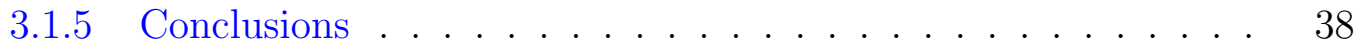

3.2 Measurement Specifications _. . . . . . . . . . . . . . . . . . . 39

3.2.1 Key Physical Parameters . . . . . . . . . . . . . . . . . . . . 40

$3.2 .2 \quad$ Sensor Parameters . . . . . . . . . . . . . . . . . . . . 43

3.3 Finalized Conceptual Design . . . . . . . . . . . . . . . . 50

3.3 .1 Sensing . . . . . . . . . . . . . . . . . . 52

3.3 .2 Geometry . . . . . . . . . . . . . . 53

4 Impact of Mechanical Piano Key Alterations on Musician Perfor$\begin{array}{ll}\text { mance } & 54\end{array}$

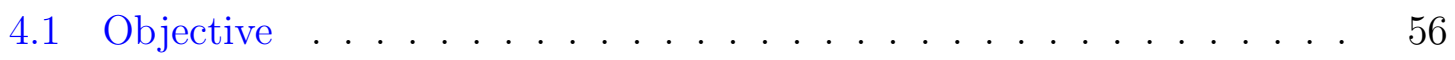

4.2 Experimental Design . . . . . . . . . . . . . . . . 57

4.2 .1 Equipment. . . . . . . . . . . . . . . . 58

4.2 .2 Subject Recruitment . . . . . . . . . . . . . . . . . 59

4.2 .3 Task . . . . . . . . . . . . . . . . . . 60

4.3 Results . . . . . . . . . . . . . . . . . . . . . . . 61

4.4 Impact . . . . . . . . . . . . . . . . . . 64 
5 Theoretical Design Analysis $\quad 65$

5.1 Definition of the System . . . . . . . . . . . . 66

5.1 .1 Coordinate System . . . . . . . . . . . . . 66

5.1 .2 Finger Contact Position _. . . . . . . . . . . . . 67

5.1.3 Strain Gauge Placement Position . . . . . . . . . . . . . 68

5.2 Theoretical Stress and Strain Analysis . . . . . . . . . . . . . 71

5.2.1 Purpose ........................... 71

5.2.2 Assumptions. . . . . . . . . . . . . . . . . . . . 73

5.2.3 Applied Stress States . . . . . . . . . . . . . . 74

5.2.4 Development of Moment Equations . . . . . . . . . . . . 77

5.2.5 Development of Stress Expressions . . . . . . . . . . . . 79

5.2.6 Theoretical Stress Matrix . . . . . . . . . . . . . . . 90

5.2.7 Development of Strain Expressions . . . . . . . . . . . . . . . 97

5.2.8 Amplification of Strain through a Wheatstone Bridge . . . . . 100

5.3 Verification of the Theoretical Analysis . . . . . . . . . . . . . . 102

5.3.1 Theoretical Strain and Voltage Outputs . . . . . . . . . . 103

5.3.2 Conclusions and Adaptations . . . . . . . . . . . . . 110

6 Final Design Assembly 113

6.1 Beam Assembly . . . . . . . . . . . . . . . . . . 113

6.1.1 Material Selection . . . . . . . . . . . . . . 113

6.1.2 Geometry Selection . . . . . . . . . . . . . . . 114

6.2 Key Assembly . . . . . . . . . . . . . . . . . . . 117

6.3 Electronics Assembly . . . . . . . . . . . . . . . . . . . 120

6.3.1 Strain Gauge Selection . . . . . . . . . . . . . . . . . . . . . 120

6.3.2 Strain Gauge Mounting and Bridge Assembly . . . . . . . . . 123

6.3.3 Data Processing . . . . . . . . . . . . . . 129 
7.1 Experimental Testing . . . . . . . . . . . . . . . 137

7.1.1 Test Design and Data Collection . . . . . . . . . . . . 137

7.2 Findings . . . . . . . . . . . . . . . . . 145

7.2.1 Conclusions . . . . . . . . . . . . . . . . . 154

7.3 Mathematical Calibration Theory . . . . . . . . . . . . . 158

7.3.1 Least Squares Approximation through Pseudo-Inverse Analysis 161

7.4 Design Output Parameters and Specifications . . . . . . . . . . 169

7.5 Future Key Integration . . . . . . . . . . . . . . . . . . . 171

8 Conclusions and Recommendations $\quad 174$

8.1 Conclusions ........................... 174

8.2 Contributions . . . . . . . . . . . . . . . . . . 177

8.3 Recommendations and Future Work . . . . . . . . . . . . . . 178

$\begin{array}{ll}\text { List of References } & 182\end{array}$

Appendix A Piano Key Physical Properties 191

A.1 Mass Properties . . . . . . . . . . . . . . . . . . . 191

A.2 Geometric Properties . . . . . . . . . . . . . . . 196

Appendix B Commercial Sensors Assessed 198

Appendix C Touch Variation Study Ethics Clearance and Questionnaire 203

Appendix D Code Listing: Verification of Theoretical Analysis Script 214

Appendix E Prototype Key Components 239

Appendix F Code Listing: LabJack Data Collection Script 245 
Appendix G Data Analysis: Experimental Data Collection

G.1 Measurement Positions . . . . . . . . . . . . . . . . 251

G.2 Plotted Voltage Outputs . . . . . . . . . . . . . . 254

G.2.1 Voltage Outputs from Fz and Fy Loading Cases . . . . . . . . 254

Appendix H Code Listing: Experimental Validation Data Collection 278

Appendix I Least Squares Approximation Matrices 285

I.1 Initial Validation of Least Square Approximation . . . . . . . . . 287

Appendix J Code Listing: Development of Calibration Matrix 292 


\section{List of Tables}

3.1 Design Input Specifications . . . . . . . . . . . . . . 50

4.1 Participant Responses Summarized of the Number of Subjects Who Identified an Applied Key-Alteration Test Case . . . . . . . . . . 62

6.1 Strength of Gauge Orientation Responses . . . . . . . . . . . . . 121

7.1 Range of Test Cases Applied During Validation Data Collection . . . 143

7.2 Final Design Outputs Compared to the Original Design Inputs . . . . 171

A.1 Experimental Data from $I_{z}$ Pendulum Testing $\ldots \ldots \ldots \ldots \ldots$

A.2 Mass Properties of Piano Key $42 \ldots \ldots \ldots \ldots$

B.1 Selection of Commercial Sensors Reviewed . . . . . . . . . . . 198

G.1 Position of $F_{z}$ Force Tests $\ldots \ldots \ldots \ldots \ldots \ldots$

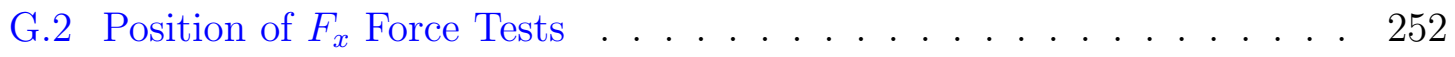

G.3 Position of Combined $F_{z}$ and $F_{y}$ Force Tests $\ldots \ldots \ldots \ldots$

G.4 Precise Mass Applied During Each Loading Case . . . . . . . . . . 253 


\section{List of Figures}

2.1 Overview of the Components of the Vertical Piano Action in an Upright Piano $[1$, edited $] \ldots \ldots \ldots \ldots$. . . . . . . . . . . . . 7

2.2 Piano Key Movement Across the Balance Rail [2, edited] . . . . . . . 8

2.3 Experimental Position of a Piano Key during a Keystroke under piano Dynamics $[3] \ldots \ldots \ldots \ldots \ldots$

2.4 Folded Beam Measurement Approach Developed by Parlitz et al. . . . 19

2.5 Experimental Setup by Kinoshita et al. . . . . . . . . . . . . . . . . 20

2.6 Experimental Setup by Grosshauser et al. . . . . . . . . . . . . . . . 22

2.7 Experimental Design Developed by Flückier et al. . . . . . . . . . . . 24

2.8 TouchKeys Capacitive Touch Sensors Installed on Piano Keyboard . . 25

3.1 Key 42 Selected for Initial Design and Testing Implementation . . . . 40

3.2 Restricting Dimensions of the Key Front (Unique to Key 42 of the Yamaha Disklavier MX1Z), with the inclusion of the front balance rail pin height . . . . . . . . . . . . . . . . . . 44

4.1 Outline of the Keys Adjusted in the Touch Perception Study with the 3-Octave Test Range Beginning on C3 (noted with the start arrow) . 58

4.2 Overview of Experimental Study Setup . . . . . . . . . . . . . . 59

5.1 Key Orientation with respect to coordinate system . . . . . . . . 67

5.2 Finger-force Application . . . . . . . . . . . . . . . . . . . 68

5.3 Position of Planes A and B with respect to the Origin . . . . . . . 69 
5.4 Strain Gauge Rosette Positions Across Beam . . . . . . . . . . . . . . 69

5.5 Stress to Strain Conversion Flowchart . . . . . . . . . . . . . . . 72

5.6 Loads on Measurement Cross-Section (x-z plane) . . . . . . . . . . . 79

5.7 Extraction of an Element at the Top of the Sensing Beam . . . . . . 80

5.8 Stress Acting on the $\mathrm{x}-\mathrm{y}$ Plane . . . . . . . . . . . . . . . 81

5.9 Stress Acting on the z-y Plane . . . . . . . . . . . . . . . 86

5.10 General Bridge Circuit . . . . . . . . . . . . . . . . . . . 101

5.11 Fz Force Variation at A1 for a Touch Position at $(0,0)$ on the Key Surface, for a Hollow Cross-Section . . . . . . . . . . . . . . . . . . . 104

$5.12 \mathrm{Fz}$ Force Variation at A1 for a Touch Position at $(0,0)$ on the Key Surface, for a Solid Cross-Section . . . . . . . . . . . . . . . . . 104

$5.13 \mathrm{Fz}$ Force Variation at B1 for a Touch Position at $(0,0)$ on the Key Surface, for a Hollow Cross-Section . . . . . . . . . . . . . . . . 106

$5.14 \mathrm{Fz}$ Force Variation at B1 for a Touch Position at $(0,0)$ on the Key Surface, for a Solid Cross-Section . . . . . . . . . . . . . . 106

5.15 Fz Force Variation at A1 for a Touch Position $100 \mathrm{~mm}$ from the yorigin position along the centreline of the Key Surface, for a Hollow Cross-Section . . . . . . . . . . . . . . . . . 108

5.16 Fz Force Variation at B1 for a Touch Position $100 \mathrm{~mm}$ from the yorigin position along the centreline of the Key Surface, for a Hollow Cross-Section . . . . . . . . . . . . . . . . . . . . . . 108

6.1 Bottom View of Piano Key, with a Front and Middle Balance Rail Hole 115

6.2 Key Angle Variation Across a Keyboard [4] . . . . . . . . . . . . . . . 116

6.3 Example of Connection Types for the Sensing Beam [5, 6, edited] . . . 116

6.4 Sensing Beam Attachment and Alignment of Pin and Bolt Hole . . . 117

6.5 Keytop Connection to the front of the Prototype Key . . . . . . . . . 118

6.6 Measured Angle for the Key 42 Prototype . . . . . . . . . . . . . . . 119 
6.7 Alignment of the Manufactured Components of the Prototype Key . . 119

6.8 RF9 Stacked Strain Gauge Rosette [7] . . . . . . . . . . . . . . . . . 123

6.9 RF9 Rosette Mounted to the Sensing Beam . . . . . . . . . . . . . . 124

6.10 Side and Bottom View of Mounted Gauges . . . . . . . . . . . . . . . 125

6.11 MIDI Bar Placement Beneath Upright Piano Keyboard . . . . . . . . 126

6.12 Wire Pathway of Ribbon Cables . . . . . . . . . . . . . . . . . 127

6.13 Wheatstone Half-Bridge Configurations for Bending/Shear Loading and Axial Loading, Respectively . . . . . . . . . . . . . . . . . 128

6.14 Assembled Perfboards . . . . . . . . . . . . . . . . . . 129

6.15 Cantilever Beam Test Configuration . . . . . . . . . . . . . . . 131

6.16 Benchtop Testing Setup for Key Prototype . . . . . . . . . . . . . . 132

6.17 F z Force Application between Unloaded, Mid-load, and High-load Applications Across Each Gauge Channel . . . . . . . . . . . . . . . . . 134

$6.18 F x$ Force Application between Unloaded, Mid-load, and High-load Applications Displaying the Voltage Outputs for One Half-Bridge . . . . 134

7.1 Defined Positions of Measurement on the Experimental Keytop . . 139

7.2 Experimental Keytop . . . . . . . . . . . . . . . . . . . . . . . 140

7.3 Angles of Measurement Used During Testing . . . . . . . . . . . . . . 141

7.4 Application of Measured Masses for Data Collection . . . . . . . . . . 144

7.5 Voltage Responses of Repeated Trials at Position 2 for the Linear Gauge Pairs . . . . . . . . . . . . . . . . . . . . . 146

7.6 Voltage Responses of Repeated Trials at Position 2 for the Rosette Gauge Pairs . . . . . . . . . . . . . . . . . . . . 148

7.7 Voltage Responses of All Bridge Outputs at Position 2, Trial 1 . . . . 150

7.8 Power Supply Reading at Each Loading Case Across Each Trial for $F_{z}$ Load Applications . . . . . . . . . . . . . . . . . . . . . . . . . . 151 
7.9 Voltage Response of All Bridge Ouputs at $-90^{\circ}$ Position, Trial 1 for the $F_{x}$ Load Application . . . . . . . . . . . . . . . . . . . . . 153

7.10 Voltage Response of All Trials for the Rosette Bridge Outputs at Angle Increment 1 for the $F_{y}$ Load Application . . . . . . . . . . . . . . 156

7.11 Pseudo-Inverse Prediction Modelled Back to the Original Data at Position $1 \ldots \ldots \ldots \ldots \ldots \ldots$

7.12 Pseudo-Inverse Prediction Modelled Back to the Original Data across all $F_{z}$ Loading Cases with the Voltage Responses from the Linear Gauge Pairs . . . . . . . . . . . . . . . . . 167

A.1 Experimental Determination of Centre of Mass Through Plumb Line

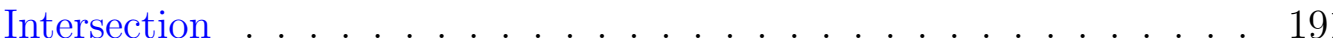

A.2 Experimental Determination of Mass Moment of Inertia Through Pendulum Testing . . . . . . . . . . . . . . . . . . 192

A.3 Verification of Pendulum Testing . . . . . . . . . . . . . 193

A.4 Experimental Determination of $I_{z} \ldots \ldots \ldots$

A.5 Setup to complete 3D Scan of a Piano Key . . . . . . . . . . . . . 196

A.6 Alignment of Key Scans to Produce a Final Model . . . . . . . . . . . 196

G.1 Defined Positions of Measurement on the Experimental Keytop . . . 251

G.2 Voltage Response of All Bridge Outputs for Each Trial of an $F_{z}$ Applied Load at Loading Position 1 . . . . . . . . . . . . . . . . . 256

G.3 Voltage Response of the Repeat Trials at Position 1 for the Linear Gauge Pairs, for an $F_{z}$ Applied Load . . . . . . . . . . . . . . . 257

G.4 Voltage Response of the Repeat Trials at Position 1 for the Rosettes, for an $F_{z}$ Applied Load . . . . . . . . . . . . . . . . . . . . . . 259

G.5 Voltage Response of All Bridge Outputs for Each Trial of an $F_{z}$ Applied Load at Loading Position 8. . . . . . . . . . . . . . . . 261 
G.6 Voltage Responses of Repeated Trials at Position 8 for the Linear Gauge Pairs, for an $F_{z}$ Applied Load . . . . . . . . . . . . . . . 262

G.7 Voltage Response of the Repeated Trials at Position 8 for the Rosettes, for an $F_{z}$ Applied Load . . . . . . . . . . . . . . . . . . . . . . . . . . 264

G.8 Voltage Responses of All Bridge Outputs at Position 3, Trial 1 for $F_{z} 265$

G.9 Voltage Responses of Repeated Trials at Position 3 for the Linear Gauge Pairs, for an $F_{z}$ Applied Load . . . . . . . . . . . . . . . 266

G.10 Voltage Response of the Repeated Trials at Position 3 for the Rosettes, for an $F_{z}$ Applied Load . . . . . . . . . . . . . . . . . . . . . . 268

G.11 Voltage Responses of All Bridge Outputs at Orientation 3, Trial 1 for the $F_{y}$ Testing $\ldots \ldots \ldots \ldots$. . . . . . . . . . . . . . . . . . . . . 269

G.12 Voltage Response of Linear Gauge Pairs from the Repeated Trials at

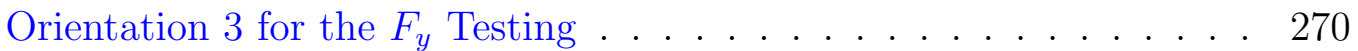

G.13 Voltage Response of Rosettes from the Repeated Trials at Orientation 3 for the $F_{y}$ Testing . . . . . . . . . . . . . . . . . . . . . . . . 272

G.14 Voltage Response of All Bridge Outputs for Each Trial of an $F_{y}$ Testing

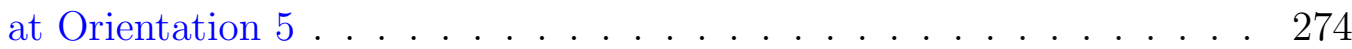

G.15 Voltage Response of Linear Gauge Pairs from the Repeated Trials at Orientation 5 for the $F_{y}$ Testing . . . . . . . . . . . . . . . 275

G.16 Voltage Response of Rosettes from the Repeated Trials at Orientation 5 for the $F_{y}$ Testing . . . . . . . . . . . . . . . . . . . . . . . . 277

I.1 Pseudo-Inverse Prediction Modelled Back to the Original Data at Position 2, with Focus on the Load Deviation in the $1 \mathrm{~g}$ Increments . . . 288

I.2 Pseudo-Inverse Prediction Modelled Back to the Original Data at Position $3 \ldots \ldots \ldots \ldots$. . . . . . . . . . . . . . 289 
I.3 Pseudo-Inverse Prediction of $M_{x}$ Modelled Back to the Original Data at Position 1 and Position 8 from $F_{z}$ Loading Tests Using the Voltage Outputs from All Gauge Pairs . . . . . . . . . . . . . . . . . . 290

I.4 Pseudo-Inverse Prediction of $M_{x}$ Modelled Back to the Original Data at Position 1 and Position 8 from $F_{z}$ Loading Tests Using the Voltage Outputs from the Linear Gauge Pairs . . . . . . . . . . . . . . . 291 


\section{Nomenclature}

DAQ Data Acquisition

MIDI Musical Instrument Digital Interface

PRMD Performance-Related Musculoskeletal Disorder

STL Stereolithography

$\left(\boldsymbol{x}_{\boldsymbol{c}}, \boldsymbol{y}_{c}, \boldsymbol{z}_{\boldsymbol{c}}\right) \quad \mathrm{x}, \mathrm{y}$, and z-coordinates of finger contact in a keystroke. These variables are unique to each keystroke.

$\left(\boldsymbol{F}_{\boldsymbol{x}}, \boldsymbol{F}_{\boldsymbol{y}}, \boldsymbol{F}_{\boldsymbol{z}}\right)$ Directional components of the contact force applied.

$\boldsymbol{y}_{\boldsymbol{A}}, \boldsymbol{y}_{\boldsymbol{B}} \quad \mathrm{y}$-coordinate of measurement Plane $A$ and Plane $B$, respectively 


\section{Chapter 1}

\section{Introduction}

\subsection{Motivation}

Expert musicians dedicate years to education and continuous practice to master their skills; with pianists being of no exception. Although playing the piano is a common activity, the physical demand of performing and the degree of fine motor control required are often overlooked. During a small 4-minute passage of a piece (a presto of Mendelssohn) 5595 notes are played, which was observed to be completed through 72 bi-manual finger movements per second [8]. Despite a musician's focus on perfecting their movement, research has found that up to $71 \%$ of professional musicians and $87 \%$ of music students will experience a performance-related musculoskeletal disorder at some point in their career [9]. To reach an expert level, a musician practices for multiple hours daily. Repetitive movements and increased demand of practice to reach a performance level creates a large toll on a musician's body, which can result in injury, disorders, or varying degrees of pain. This thesis is interested in understanding the biomechanics of performance. The general term of performance-related musculoskeletal disorders is applied to encapsulate the varying effects of pain and injury during performance. 
Performance-related musculoskeletal disorders (PRMDs) have been categorized as, "any pain, weakness, numbness, tingling, or any other symptoms that interfere with your ability to play your instrument/perform at the level to which you are accustomed" [10]. Extensive research has been made to identify the prevalence and impact of these disorders on pianists; as well as establish an understanding of the causes of PRMDs. The act of playing the piano involves an intricate relationship between the pedagogical approach of teachers, the biomechanics of a pianist's movement, and the piano itself. These relationships and the cause of PRMDs experienced by pianists can be multi-factorial; however, the basis is often biomechanical. The critical feature of the above-mentioned relationship is that of the interaction between a mechanical device of the piano action with a biomechanical system of the hand and arm.

Unfortunately, the biomechanics behind playing is still not fully understood. This has left musicians visiting multiple physicians with no long-term solution to their PRMDs [11-13]. The level of demand for aid in PRMDs today has reached a point in which specialized clinics for musicians have become available [14]. Playing-related disorders not only impact a musician's ability to play, but can also cause chronic pain which is severe, incapacitating, and beyond their control. This impacts a musician's life physically, psychologically, and financially $[9,15]$.

Parallels can be drawn between elite athletes and musicians. Athletes focus their lives on their career, continuously improving skills for competition and maintaining their health to allow optimal performance. For professional athletes, this can be achieved through a support team who have a thorough understanding of the biomechanics of the body in sports. The same level of focus can be said about expert musicians who train and perform at an elite level, with a high risk of developing an 
injury. Despite the biomechanics research in other fields of elite performance however, there is a clear lack of understanding of musicians' movement which has left pianists without solutions and methods of minimizing the risk of PRMDs in performance. Musicians continuously risk PRMDs to maintain the technical perfection of their pieces. In doing so, they also hinder their health, livelihood, and in the case of many students, the possibility to graduate. It is clear an evaluation of the interactions that may be leading to PRMDs and possible preventative measures are required.

\subsection{Problem Statement and Contributions}

It is recognized that the lack of understanding of the interaction of the biomechanical and mechanical systems of the human arm and piano action, has limited the progress of research in piano performance and PRMD prevention. The overall goal of this research was to investigate the interaction at the base level; the interaction between the piano key and pianist's finger during a keystroke. At this base level, the aim of the research was to discretely collect accurate force and position profiles during unique keystrokes.

This thesis proposes a prototype piano key to achieve the aforementioned measurements. Specifically, it outlines the design and assembly of a prototype key that assessed the ability to measure the force applied to the key surface. This stage of research was focussed on developing a hardware design to create the first stage of prototyping. Issues related to advanced calibration approaches that optimally integrate the measurements are beyond this initial prototype development. This research was developed to provide these solutions while also contributing a new approach to musician biomechanics assessment. This stage of the design will not assess key movement measurement, as extensive methods already exist currently. Discrete collection 
of both the positions and force applied during a keystroke to initiate the piano action process allows analysis of variation in forces and moments developed during the unique interaction. These methods could also be utilized in measurement of other motor tasks. These measurements would provide invaluable information for future analysis of piano performance, teaching, rehabilitation techniques, and the analysis of the interaction which can lead to pianist PRMDs.

\subsection{Thesis Outline}

The following chapter, Chapter 2 provides a description of the interaction of the piano and pianist to establish an understanding for those unfamiliar with the relationship. The chapter also summarizes previous work which has been completed throughout various disciplines to build an understanding of musician movement and injury.

Chapter 3 outlines possible experimental approaches to attain the set research goals. The development of the functional specifications of the design is further outlined in this chapter.

The impact of the design specifications on the integrity of the research study is further explored in Chapter 4. This chapter specifically looks at piano key mass properties, and identifies if and to what level a variance from original properties is acceptable through an experimental study.

Chapter 5 outlines the theoretical analysis that was completed to verify that the final design would meet the measurement specifications. 
An overview of the final design is provided in Chapter 6. This chapter also described the details of the design assembly, and the hardware used to complete data collection.

The data validation process is described in Chapter 7. This includes the experimental process used to validate the final outputs from the prototype were related to the applied loading conditions on the piano key.

Final conclusions, assessment of the study contributions, and recommendations for future work for this research is outlined in Chapter 8, which concludes this document. 


\section{Chapter 2}

\section{Review of Literature}

As Chapter 1 illustrated, development of injury prevention for musicians requires further understanding of the biomechanics of their movement. To reach a solution, a basic understanding of a pianist's movement and the range of their injuries is beneficial. As this research aims to develop a method of measuring the pianist-key interaction, the chapter additionally provides a summary of previous research in this field, including the various methods of pianist movement assessment.

\subsection{Piano-User Interaction}

The invention of the piano can be dated back to the early 1700s. Moving forward from the harpsichord, the piano provided increased musician control of the instrument to add dynamic sound level to performance [1]. Although the piano has had some developments since its early invention, and can now be found in different variations, the main components have remained the same. The sound created by a piano is the resultant of strings vibrating. This is made possible through the action mechanism of the piano. 
The action assembly of a piano consists of the piano key, the escapement, and the hammer, shown in Figure 2.1. During a keystroke, a struck piano key triggers the movement of the escapement which is in contact with the back end of the key. The downward motion of the front of a key during a keystroke results in an upward motion of the back of the key. The upward motion creates the contact and transfer of energy to the escapement key action. The action component then transfers the force applied at the key front to the key hammer. When triggered, the hammer moves in free-flight until it strikes the respective string, causing the string vibration [1]. The focus of this research is to measure the biomechanics of performance at the base interaction. Therefore, this research assessed contact force on the surface of the piano key that causes the action to move.

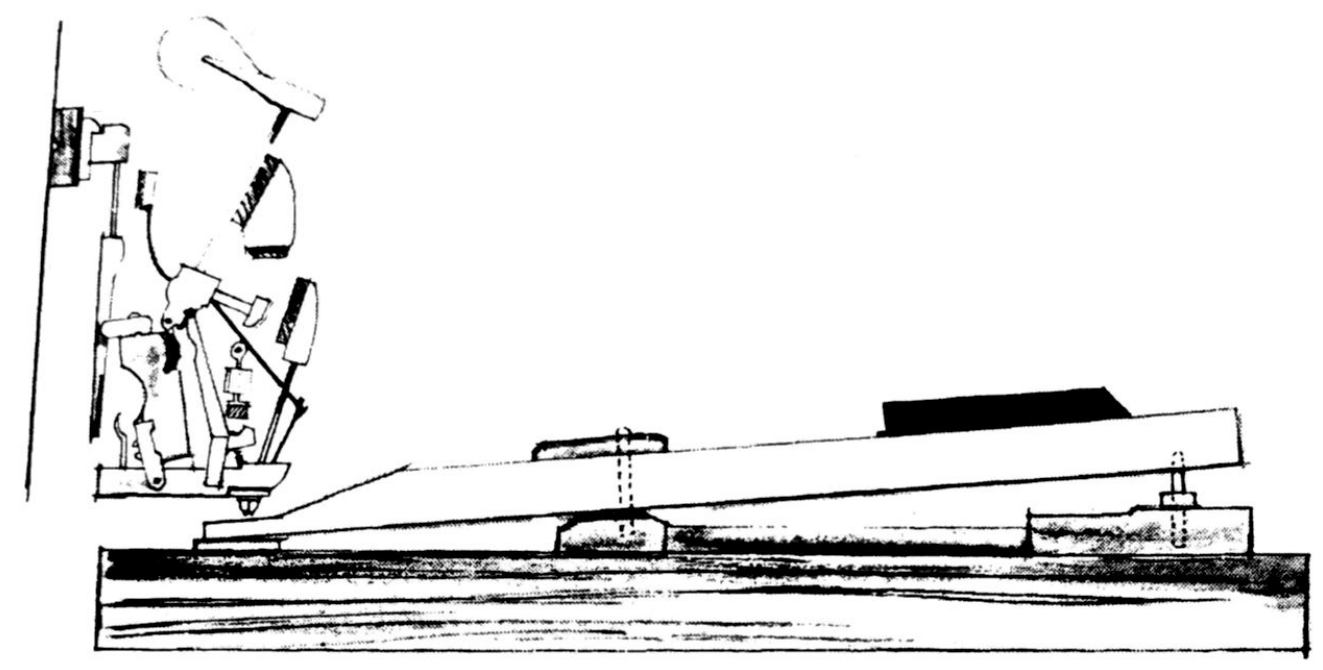

Figure 2.1: Overview of the Components of the Vertical Piano Action in an Upright Piano [1, edited]

As Figure 2.1 displays, the front portion of a piano key that is visible to a pianist represents only a fraction of the entire key. The movement of the full key is guided by two points on the key. The alignment of a full piano key is constrained at the 
front and middle portion of the key. The front pin in the piano keyboard, known as the front rail key pin, ensures the key is constrained from lateral movement. The middle pin in the piano keyboard, referred to as the balance rail key pin, also serves this purpose [1]. These pins restrict a piano key to purely vertical movement. During a keystroke, the key is restricted to a vertical travel of $6-8 \mathrm{~mm}$ [1]. In addition to providing movement constraints to the system, the balance rail also acts as a pivot point to the piano key, see Figure 2.2. Therefore, a simplification of a full key can be equated to that of a first class lever. The effort applied during a keystroke is translated to a motion from the escapement, and in turn the hammer of the action mechanism.

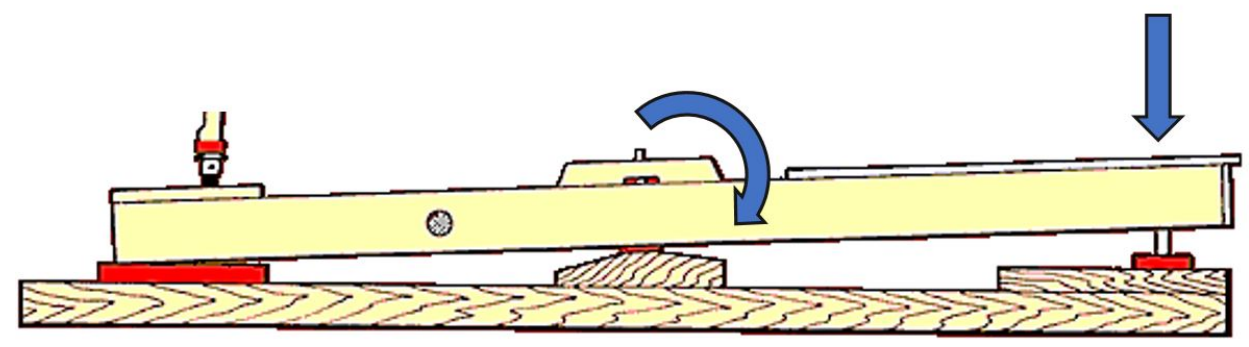

Figure 2.2: Piano Key Movement Across the Balance Rail [2, edited]

Execution of a keystroke depends on the variation in force exerted on the key. Key touch can be divided into three main events. Touch initiation is defined as the beginning of force exertion on the key. When the downward motion of the key reaches the keybed, a force changed noted as keybed impact occurs. During keybed impact, the contact of the key and keybed results in a peak in the key reaction force as the key is stopped as it meets the keybed. This is the lowest point in the key trajectory. Additional variation in the force can be the resultant of internal impacts from the action mechanism of the piano. During that time, touch release occurs in which the pianist removes their finger from contact. The variation in length of these events 
is dependent on the piece being played as well as playing style [16]. An expectant normal force curve has not been accurately depicted from previous research for the events in a keystroke. However, under defined dynamic and tempo requirements, the position of the key during these events has been studied by various research teams. An example of the key position may be represented as Figure 2.3.

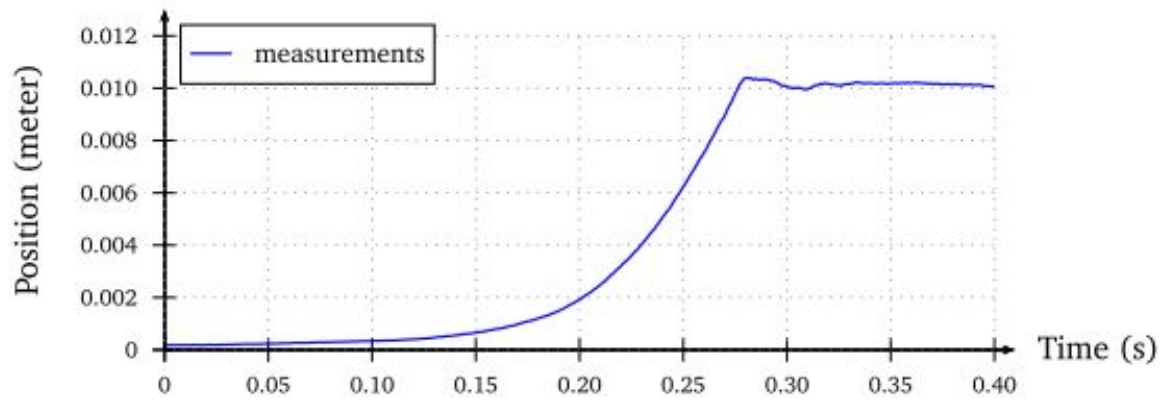

Figure 2.3: Experimental Position of a Piano Key during a Keystroke under piano Dynamics [3]

The aforementioned effort applied during a keystroke can be unique to each musician, the passage played, and the actual instrument itself. During a keystroke, two main types of touch may be applied. Percussive touch, also referred to as struck touch, is defined as when the finger started above the key. Non-percussive touch, commonly referred to as pressed touch, is defined as when the finger begins from rest on the key [17]. The minimum force required to initiate the motion of the key can vary between piano models and within the keys across a keyboard themselves. The down weight, which is isolated to the weight that is required to begin key movement, typically ranges between 50-55 grams [1]. Additional weight is further required to overcome the resistance of the action at the end of the key, allowing the full key motion upon application. Therefore, a pianist will exert a greater force on the key while playing. The range of this force will be further investigated in Section 2.3. Looking 
purely at the physical interaction of the finger and piano key, the force applied to depress the key is characterized by the magnitude of the force, the direction of the force, and the position in which it is applied on the key. For pianists, their repertoire can require a key to be struck from different angles, which results in variation of the force vector applied [17].

Previous key movement analysis has provided insight on the length of time and movement of the key during a keystroke. Modern measurement techniques have allowed more advanced research on key movement. Goebl and Bresin, [18], completed research to measure the variance in different types of touch on piano keys. Measurement of key movement was completed through the use of accelerometers attached to the key. Their testing revealed that key travel time during varied keystrokes is independent of pitch, but rather greatly influenced by pressed or struck touch and musical dynamic. The study concluded that the time from finger contact to key bottom ranged from 20 to $200 \mathrm{~ms}$, with the variance due to the factors of touch type and dynamics. This research provided a wider range in key bottom time compared to previous studies as it spanned a wider musical dynamic range. Askenfelt and Jansson's, [19], more robust method cited a key travel time ranging from $25 \mathrm{~ms}$ to $160 \mathrm{~ms}$ for forte and piano tones, respectively.

\subsection{Musicians and PRMDs}

It is not the purpose of this literature review to extensively examine all research in relation to musician PRMDs, but rather to provide further context to the current issues which has led to the development of this current thesis research. Performancerelated musculoskeletal disorders further encompass overuse syndrome, tendinitis, and

nerve compression syndrome [14]. These forms of musculoskeletal complaints can lead 
to severe long-term pain and disabilities, which can be career-ending for a musician. When considering the posture and movement of a pianist during a performance, one can visualize the impact practice and performing could have on the body, specifically the potential for musculoskeletal disorders, including overuse syndrome. Overuse is often described as passing the point of muscle fatigue. These issues can develop due to factors such as intensive practice, psychological factors, poor posture, non-ergonomic technique, excess force when playing, general overuse, and occupational factors such as a change in repertoire programming or a change in musical instructor $[9,20,21]$. However, for an expert musician, the mitigation of the occupational risk factors is outside of their control. Furthermore, in the competitive performance environment, a decrease in practice or performance time to treat a PRMD may draw unwanted attention. Ultimately, these factors can be used to describe not only injuries of musicians, but common workplace injuries. Early reports on occupational injuries cited that injury could occur due to, "some particular posture of the limbs or unnatural movements of the body called for while they work" [22]. Although issues of occupational injury have been documented since the 1700s, the concept of musicians injury and the development of the concept of PRMD was not truly studied until the 1980s [23].

A number of publications on musician musculoskeletal disorders have been released since that time, with interest growing in various disciplines. Attempts to develop an understanding of the prevalence of PRMDs amongst musicians have been completed through case studies and qualitative questionnaires [9, 12, 24-26, for example]. A prominent study from Zaza developed an understanding of the musician's perception of injury. The study established that contrary to normal pains, PRMD symptoms present as chronic and severe to a point where playing is negatively affected and the symptoms are no longer within a musician's control [9]. An overview of literature on the subject of musculoskeletal complaints in expert musicians reported that the 
impact of these disorders were predominately reported by musicians to have a lifetime prevalence. The range of lifetime prevalence reported by the musicians in the studies ranged from $62 \%$ to $93 \%$ [15]. The onset of these PRMDs is not limited to professional musicians. A study of piano performance majors also found that $86 \%$ of students experience pain when playing. The statistical analysis across the musician evaluation studies varied widely. However, this can be justified by the variation in data collection, the populations surveyed, and the individual interpretations of nonplaying related pain and playing-related pain.

Research has and continues to report a stigma around PRMDs. Expert musicians work in a competitive environment, where proficiency is judged during each performance. An incapacitating PRMD can threaten a musician's career. Case studies which have surveyed musicians have found that they are inclined to hide their injury. This has led to situations where musicians play while in severe pain during rehearsals and concerts [27]. Multiple reports on musician injury have cited the concept that pain is inherent to succeeding as a professional. Musicians stated opinions from peers such as 'injury being a sign of inferior talent', that 'pain and endurance are a sign of a dedicated musician', and that there is a general denial of pain in the music community. These opinions have been cited across both surveys of professional and student musicians $[12,23,25]$. When piano students were surveyed, it was concluded that they received limited education in PRMD prevention. However, they had indicated a high level of interest in education to prevent further issues [28]. The conclusion from multiple researchers has noted that an understanding of PRMDs must not only be made to help provide prevention for musicians, but also an awareness for teachers to develop methods for students to provide strategies for future teaching and rehabilitation methods. 


\subsubsection{Importance of Studying the Biomechanics of Finger Contact}

The lack of awareness surrounding PRMD and their prevention can be related to the disconnect of understanding of the biomechanics behind playing the piano. Current pedagogical approaches focus on metaphorical interpretations of weight, energy, and speed to provide guidelines to proper practice and performance. However, these concepts are not supported through the physiology of playing. Although various teaching and recovery methods have attempted to address playing-related issues, they have not yet reached a solution. Research in relation to the biomechanics of playing and its connection to PRMDs is therefore critical to adapt current pedagogical approaches [23]. Surveys with student pianists identified that there is little knowledge about the physics and physiology of performance due to lack of exposure to the material during their training program [29]. This lack of knowledge can impact the ability to perform. Pianists do not have a complete understanding of how their movements can impact the tone while they play or if their movements and fingering strategies can result in injury or a PRMD.

The variation in playing technique can be the result of unique configurations of the body during playing. Different postures and positions, including the curvature of the fingers, angle of the wrists, and position of the elbow, can all contribute to the motion which lead to key depression. Although these unique configurations among musicians may produce the same tone, the loads on the joints and the muscle groups activated to complete the key depression may vary. Muscle activation required for piano playing typically utilizes the smaller muscles of the hand and forearm which allows fine motor control. A less "efficient", but possible option, is to also use the larger muscles. Motion of the arm and hand is possible through the activation of 
multiple muscle pairs, to not only initiate motion but to counteract other movements. With the high level of variation possible among a musician's playing configuration an assessment of the finger-key interaction is critical in assessing the possibility of injury, fatigue, and other PRMDs.

Although unique configurations and postures are apparent during playing, previous methods of keystroke force measurement often make the simplifying assumption that the finger-key interaction is a purely vertical motion. However, pianists often achieve key depression through a motion in which the finger pulls down on the key. From a biomechanics perspective, a motion in which one pulls in a direction where the motion does not occur is known to be, almost always, a more efficient movement. When interacting with a constraint, the use of additional muscular effort to control the direction of the contact point, rather than the constraint, can be inefficient [30]. During a keystroke finger contact can result in high internal forces in the finger and hand [31]. Repetitive practice and performance which result in these high forces require further understanding to prevent musculoskeletal problems in pianists.

\subsection{Previous Measurement Techniques}

Development of a measurement technique first requires an overview of past and current measurement methods that have been developed and used in musician biomechanics research in the past. The various approaches, and most notable advances in this research, have been summarized below.

\subsubsection{Pianist Movement Analysis}

There is limited early research on the biomechanics of movement and the force applied during piano playing. Marie Jaëll and Charles Féré completed early research in 
the physiology of touch in 1896 [32]. The methodology of the study involved applying ink to a participant's fingertips to assess the uniqueness of touch among different musicians playing the same piece. The results allowed Jaëll to identify the variation in finger position across different musicians. Their early research established that the patterns of fingerprints could be classified between experienced and inexperienced pianists.

In the 1920s, Otto Ortmann conducted more extensive research on musician movement. His works have been credited as the first technical approaches of examining technique and movement, with views from both the fields of physiology and engineering [33]. Ortmann aimed to identify the relationship between force and method of touch, struck or pressed, during a keystroke. Identification of touch type was achieved through a biomechanical approach. Ortmann attached levers to defined points on the fingers of participants, which during movement created lines on a paper roll. This allowed recording of hand and finger movement. Ortmann further attempted to investigate the finger-key interaction through analyzing key-depression. Their rugged method utilized isolating a piano key and using a smoked piece of glass to track the frequency of a tuning-fork. The resultant output of the tuning fork allowed a visual change as the speed of the key varied. Ortmann used the relationship of force and acceleration to represent the change in key depression through different force applications [17]. These research methods allowed early identification of the variation of touch during a pianist's performance.

Multiple studies have implemented methods to collect information on the movement of a pianist's wrists, hands, and fingers. For example, an attempt to identify the range of motion of the wrist of a pianist was completed by Chung et al., [34]. 
They approached this with the use of biaxial electrogoniometers. This method required attachment across the wrists of the musicians, using straps which required adjustments throughout testing. The research was able to identify the variation in range of motion between different playing styles. Another method commonly used is the use of surface electromyography, which detects muscle activity. An application of sEMG with pianist research was completed by Bejjani et al., [35]. Their research defined patterns of muscle and joint strain, in relation to different playing posture methods. Their research also utilized motion capture to further identify movement. From literature, it was noted that the main drawback of sEMG measurement is the difficulty in discerning activation signals across the multiple muscles of the arm [36].

Motion capture methods have commonly been used in applications in which posture, or comparison of movements across a test group are desired. A study by MacRitchie and Bailey, for example, used passive UV paint markers painted directly to the hands of a pianist. The placement of a UV light above the piano keyboard allowed measurement of hand position along the key surface with respect to the horizontal movement across the keyboard and the movement towards the body of the piano during performance [36]. The study additionally completed a survey of pianist movement literature. They concluded that researchers used both passive and active motion capture methods. Popular passive methods included motion capture using Kinect devices, infrared markers, and paint markers. Active devices applied have included LED position sensors, accelerometers, and electrogonimoters. Benefits of motion capture systems are noted as the ability to apply this technology in most performance spaces. However, the downfall of this method is the possible occlusion of markers. This has been due to the nature of movement of a pianist during performance. These methods have often been used in literature with additional measurement methods to further track movement, position, and force measurement of 
musicians during various playing tasks.

\subsubsection{Pianist Finger-Key Force Measurement}

Another approach to understanding the biomechanics of playing has been through measuring the force applied during playing. Simulations and mathematical analysis have allowed indirect measurement of force. Harding et al., [31], aimed to measure finger tendon tension and joint reaction forces for a keystroke based on the finger position and finger force used by a pianist during varied key strikes. Specifically they attempted to develop a mathematical model of the finger based on finger anatomy with the inputs of the finger position and force measured on the key. The force applied to the key was measured indirectly through measurement of the normal force and calibrating these values with respect to the key velocity. A piezoelectric force transducer was affixed to the top of a piano key. The data which were collected allowed force calibration across the keys. The MIDI interface of the piano collected key velocity data. From the data collected, mathematical models relating the key velocity and internal forces were developed. These models were then solved in an optimization routine to determine the optimal finger position to minimize internal forces. Overall, across the four participants, they noted a maximum peak force was detected upon full key depression. In comparing the internal force across different finger positions, it was concluded that a curvature in the finger reduces internal joint forces. Later research by Harding further used this method with infared position detectors attached to the finger and piano key [37]. This combined method provided further insight on the position of the finger. This research opened a discussion on posture and its potential to reduce musician injury.

Piano force and position theoretical models have been studied more recently, such as those from Thorin et al. [38]. Their work aimed to assess the dynamic behaviour 
of the piano key and action to develop theoretical models. They developed various models to compare their expected results against force and motion measurements through a real-playing scenario. Their work identified that proper identification of the finger-key force interaction has not yet been properly achieved. In the validation of their model, they required accurate key contact force measurements to compare the experimental and model values. Their experimental setup completed testing with an axial piezoelectric force sensor applied on top of the tip of a piano key. This allowed normal force measurement. Additional optical and position measurement was made for the hammer an action to allow verification of the accuracy of their models. Testing was completed to compare simulations in response to force and motion controlled simulations. This initial setup, and their model completion identified peak force ranges from $4 \mathrm{~N}$ to $15 \mathrm{~N}$ for piano and forte musical dynamics, respectively.

Early work in direct force measurement during a keystroke can be credited to Parlitz et al. who attempted both mechanical and biomechanical approaches. One mechanical approach was aimed to identify a correlation between pianist skill and force applied in a keystroke during coordinate tasks [39]. Their approach utilized a sensor-matrix-foil which was positioned on the keybed of the piano beneath five adjacent keys in place of the felt which is normally placed between the key and keybed. The method allowed force measurement of five keys simultaneously when a key is fully depressed. Participants were instructed to complete tied-finger exercises, which required participants to hold down some keys while the remaining completed the required keystrokes. They concluded that expert pianists had a higher level of independent coordination of playing. Quantitative results of their force testing reported a vertical force range from $5 \mathrm{~N}$ to $20 \mathrm{~N}$, with respect to the musical dynamic range of playing tasks. They concluded that this was an effective method, but the instrumentation used only had a minimum detectable force of $2 \mathrm{~N}$ which was not 
appropriate for this application as a significantly lower force range can be applied to the key to initiate a keystroke. Earlier work by Askenfelt et al. cited a higher force range in their study, [19]. Although the details of their measurement process were not explicitly provided, their comparison of touch found peak forces were detected at up to $50 \mathrm{~N}$ at a fortissimo dynamic range.

Later work by Parlitz, combined approaches of 3D motion measurement and force measurement [40]. An augmented piano key with an internal folded beam affixed with strain gauges at two positions along the beam, provided a new measurement method that was independent of force application position. The folded beam approach established a method to measure vertical force and position along the key, outlined in Figure 2.4. Additionally, Parlitz looked to achieve 3D motion measurement through sensors along the pianist's hand and arm. Although the results of the measurements were not provided, this research did provide some insights on measurement techniques. The approach used combined a biomechanical and mechanical approach to identifying finger-key interactions.

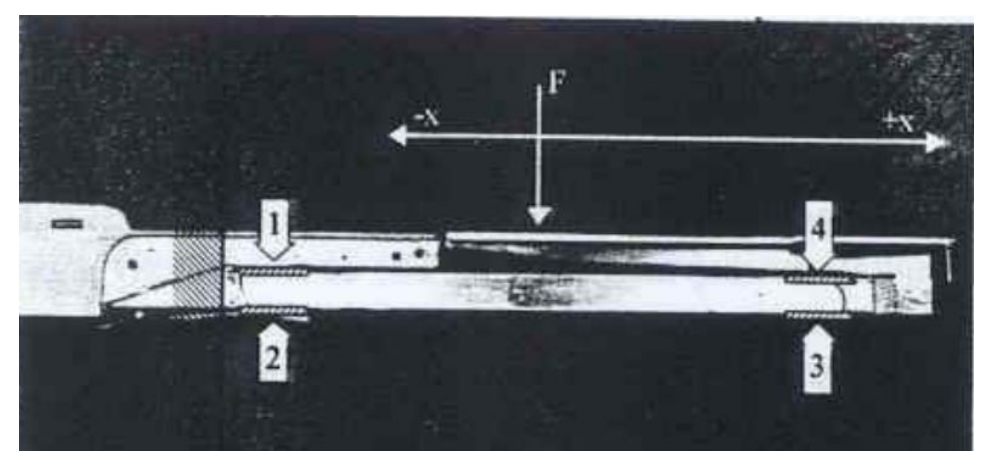

Figure 2.4: Folded Beam Measurement Approach Developed by Parlitz et al.

Additional early attempts of keystroke force measurement were made by Riehle et al., [41]. Rather than altering the piano to allow measurement, their approach 
required placement of an entire piano on a force plate. Measured changes in force were therefore considered the vertical force applied by a pianist during playing. As total force was measured, this technique considered the force produced by both the left and right hand. The research team additionally used video measurement to understand the movements of the wrist and hand of the participants during testing. They concluded that the method was effective in measuring the total force applied, which was noted to increase with musical dynamic expression. The recorded force applied ranged from 100-200 $\mathrm{N}$ at a forte dynamic level and 300-500 $\mathrm{N}$ at a fortissimo dynamic level.

The application of a force transducer to obtain finger-key interaction data was continued by Kinoshita et al., [42]. Their study aimed to develop a relationship between vertical keystroke force and the loudness of the generated tone. To achieve this, three types of instrumentation were used. To complete tone measurement, a sound level meter was fixed above the key. To obtain information on key position throughout a keystroke, an LED position sensor was applied. Finally, force was measured with the use of a uniaxial strain gauge based force transducer. The transducer, displayed in

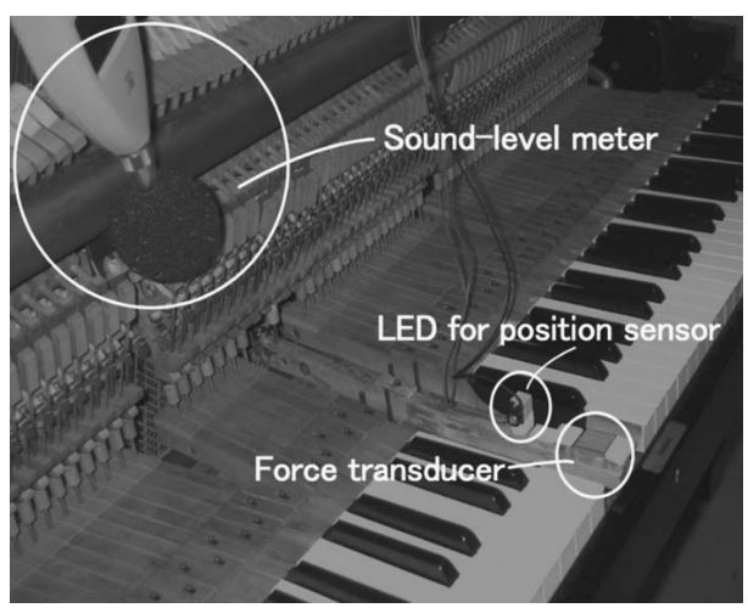

Figure 2.5: Experimental Setup by Kinoshita et al. 
Figure 2.5, was noted as not affecting the interaction of the pianists with the key to allow measurement of the normal force. Through examination of struck and pressed touch the research identified that the relationship between force and level of piano tone could be described through an exponential function. Overall, they noted a maximum average force application range between $3 \mathrm{~N}$ at a pianissimo dynamic and $60 \mathrm{~N}$ at a fortissimo dynamic level. The results of the study indicated that the maximum vertical force applied from their participants occurred before the moment of maximum key displacement, which contradicted previous studies and hypotheses on the force application. The research team justified this finding with the conclusion that hammer action of the key begins movement before key bottom occurs which justifies the offset of force application on the key. The study did note that assumption of pure vertical force application dismissed the measurement of directional forces.

Collaborative work by Furuya continued through further investigation with this experimental setup. Additional testing compared musical dynamics which noted that piano levels did not exceed a vertical force of $5 \mathrm{~N}$ while forte reached a peak reaction force of $24 \mathrm{~N}$ [43]. Further analysis with this setup involved inclusion of motion capture methods. Methods such as EMG measurement of the arm [44], inverse dynamics of upper limb movement with LED motion capture [45], and measurement through a sensor embedded glove $[46,47]$ have been utilized. These methods allowed further insight on arm movement and joint kinematics. Specifically with the application of a sensor embedded glove, data collection provided finger movement and hand posture data through a wearable measurement method. Force measurement methods have more recently been used to compare key forces and their relationship with tempo and dynamics between experienced and inexperienced pianists. Oku and Furuya, [48], completed force measurement with a uniaxial force transducer fixed to the end of the key. Experimental playing tasks at different dynamics noted that forces detected 
did not exceed $10 \mathrm{~N}$. Their research affirmed previous findings which identified that keystroke force increases with loudness. Through comparison of piano experience, it was concluded that in terms of keystroke force, a difference was only significant at louder tones. However, during repetitive tasks, the force applied was more consistent across the experienced test group. This research identified force control among pianists during playing.

Keystroke force and position measurement has also been previously collected from the perspective of the upper extremities which contribute to the total force outputted at the fingers. Research completed by Grosshauser et al., [49], used wearable accelerometers and 6-axes sensors to achieve their measurements. Their experimental setup, shown in Figure 2.6, attempted to collect wrist and hand force and torque data to relate this to keystroke force through a sensor fixed to the front of the keyboard. Accelerometers on the participants finger allowed additional position and acceleration data. Their measurement of applied hand force over a keystroke recorded the vertical component of force remaining under $20 \mathrm{~N}$, with a minimal force contribution in the perpendicular axes.

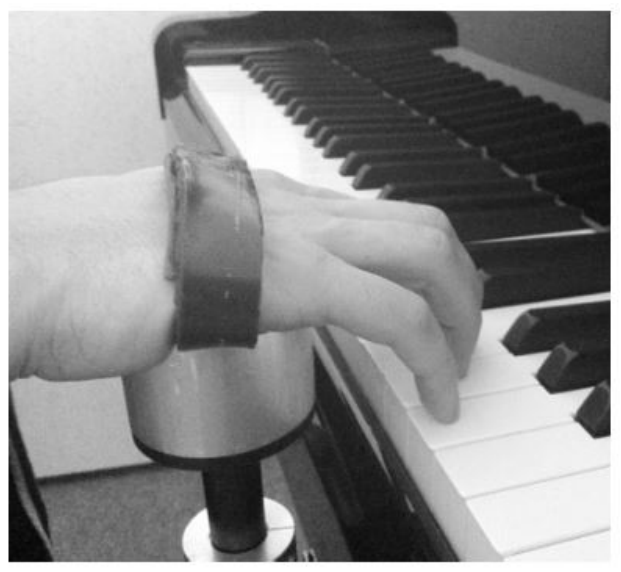

Figure 2.6: Experimental Setup by Grosshauser et al. 
Finger force measurement has also been applied to other musical instruments. Use of flexible force sensitive resistors has been applied to both piano keys and violin fingerboards by Grosshauser et al. [50,51, for further reading]. The sensor matrix applied to a piano key or violin fingerboard, provided force and position collection with a $40 \mathrm{~g}(0.39 \mathrm{~N})$ sensitivity. The matrix, although visually apparent to participants, did provide a less obtrusive method of data collection. This approach was later further integrated with their research of violinists by also integrating 3 uniaxial load cells in the fingerboard of a violin to collected additional force data.

A more recent attempt at measurement of the normal force during a keystroke was attempted by Flückiger et al. [52]. They attempted to measure the normal force during a keystroke with an unobtrusive measurement method. Use of a commercial strain gauge based load cell was implemented. A single 1-axis load cell had a measurement range from $0 \mathrm{~N}$ to $40 \mathrm{~N}$ with a resolution of less than $8 \mathrm{mN}$. With their additional instrumentation, they were able to reach a sample rate of $1000 \mathrm{~Hz}$. To achieve unobtrusive measurements, they applied their load cell within the piano key. Their methodology required a piano key to be cut to remove material, as shown in Figure 2.7. The removed material was then replaced by a 3D printed support to which a load cell was mounted. The deflection of the top portion of the key then allowed force measurement as contact was made with the load cell. It was noted that leakage of force, and therefore inaccurate measurements, was present due to the continued wooden connection of the piano key. Bench-top testing was completed under compressive force application. Their research noted that although the approach provided a method of unobtrusive normal force measurement, it did not encompass all of the necessary measurements to investigate finger-force interaction. 


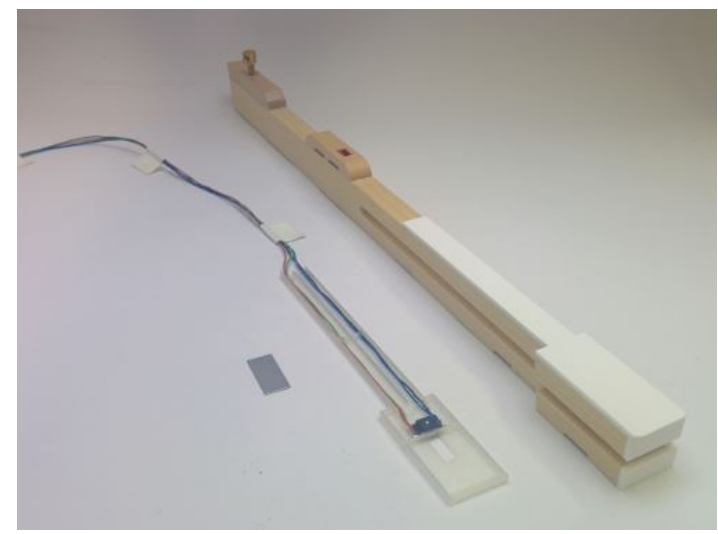

Figure 2.7: Experimental Design Developed by Flückier et al.

Focussed movement measurement of the hands of musicians has been thoroughly studied by McPherson et al.. Their earlier research identified methods of continuous key angle measurement. These measurements were additionally used to derive information on the key velocity, percussiveness, and aftertouch applied from the pianist to the keybed [53]. The instrumentation of this research remained unobtrusive to pianists. Adaptations were applied to a pre-existing concept of a MIDI data bar which implemented an optical sensor strip along the back of the keyboard. An improved scanner allowed continuous measurement through a redeveloped optical sensing design LED and photo diodes. This technology had also been applied in conjunction with other devices used to track touch position and contact area of the fingertip during a keystroke $[53,54]$. Motion capture methods paired with a camera suspended over the piano provided further details on key movement and the $\mathrm{x}$ and $\mathrm{y}$ position of the fingers during piano playing tasks.

McPherson further developed methods to measure finger position and finger contact information. They developed capacitive key sensors that could mount to the surface of a piano key, commercially referred to as TouchKeys $[55,56]$. Capacitive multi-touch sensing provided unique touch start and note release information. The 
white keys were designed with 25 sensor pads, which added $5 \mathrm{~g}$ and $1.6 \mathrm{~mm}$ in height to the key, see Figure 2.8. However, it allowed additional measurement of the position of touch on the key surface, with respect to a 2-axis coordinate system defining the movement across the width and length of the key surface. It was noted that throughout the narrow portion of the key, the position associated with the key width was not measurable. Additionally, contact area was calculated through a centroid calculation between the activated sensors. Measurements were collected every $5 \mathrm{~ms}$ to allow data collection during playing tasks. This sensor development allowed further research to be completed not only for pianist movement analysis but also key movement for indirectl finger-key force measurement.

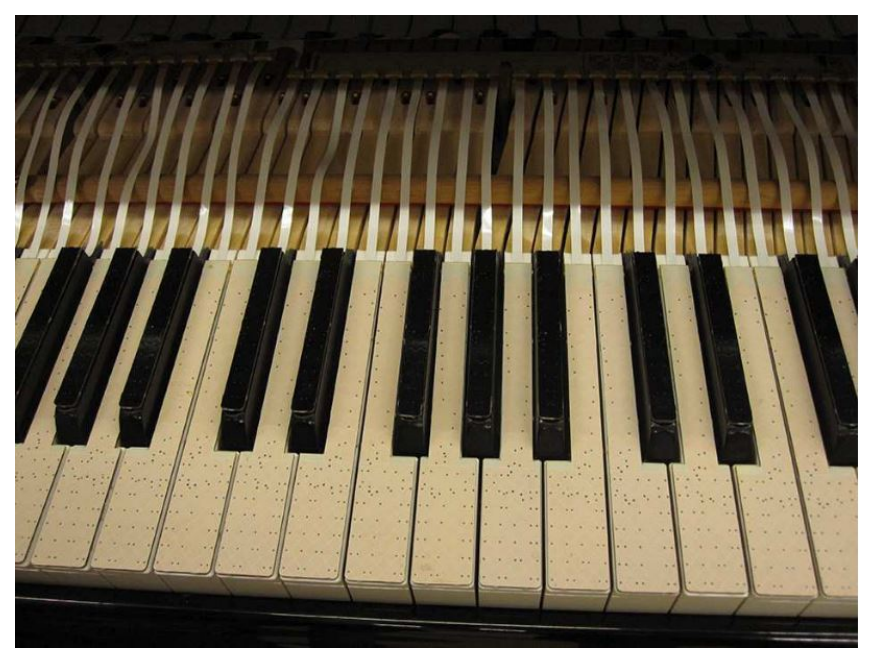

Figure 2.8: TouchKeys Capacitive Touch Sensors Installed on Piano Keyboard

The PCB-based sensor created by McPherson is not the first of its kind for piano advancements. Moog developed Multiply-Touch Sensitive Keyboards, [57]. Their design developed keys with sensors to allow detection of the position of the key during movement, as well as the $\mathrm{x}$ and $\mathrm{y}$ position of the finger when in contact with the key. Similar to McPherson, they developed a resistive film capacitive sensor across the keys. Additionally, they made adjustments to a key-bed to place 1-axis force sensors 
under the front rail of each key. This allowed additional force information during the bottom contact of the key. These methods of measurement have not only been applied in research applications, but also in that of musical expression. Lamb's use of these applications have developed technology such as the ROLI Seaboard [58]. The Seaboard was designed to allow pitch bending and other musical expression through a keyboard design. They achieved this through iterations such as force-sensing resistors to allow finger position and contact area detection for the use in vibrato and musical expression.

\subsection{Summary}

A review of early and more recent research in the area of pianist biomechanics clearly indicated that a lack of clarity in measurement was still present at this time. The review demonstrated that various methods of data collection had been attempted. An assessment of the methods to approach measurement based off of these findings was required before moving forward with a design. 


\section{Chapter 3}

\section{Design Approach}

This chapter outlines the decisions made to produce a finalized conceptual design. Section 3.1 reflects on the previous methods of data collected used in finger-key force measurement, as described by Chapter 2, and determines the appropriate methods of data collection for this application. Section 3.2 describes the specifications for both the overall design and the measurement instrumentation. Finally, Section 3.3 provides a general description of the conceptual design that was developed after completing these design review steps.

\subsection{Evaluation of Potential Design Approaches}

The intent of the current research was to develop a method to collect accurate force profiles during individual pianist keystrokes. The set research goals could be achieved from various methods, which was clear through review of previous literature. To ensure the most effective method was selected, the main approaches to capturing the necessary data were reviewed and limitations were evaluated. 


\subsubsection{Biomechanical Approach}

As a keystroke involves the interaction of a biomechanical and mechanical system it is possible to analyze the problem from a biomechanical perspective. Piano technique requires both precise small-scale motion of fingers on the key surfaces and planned large-scale movement of the hands and arms. Chapter 2 summarized various methods used in previous works to collect these measurements.

Biomechanical approaches used in previous studies have varied. One common method, which was previously described, is that of motion capture measurement to collection posture and position data of participants. This approach can vary in equipment used, including the use of reflective markers, UV paint markers, LED markers, and high speed cameras [36,45, for example]. Another commonly referenced method is the use of sEMG to detect muscle activation during playing tasks [35, 44, for example]. Both of these methods would be unable to directly measure force application, but could be used for indirect or paired measurements. In general, these methods are advantageous as they provide continuous movement measurements and can be applied in most testing environments. These measurement methods also carry disadvantages.

In general, these methods are not user friendly as they require a knowledgeable researcher to accurately set up markers on a participant and complete recordings. Biomechanical testing requires focussed researcher setup and calibration due to the variability between participants. This is necessary to ensure reproducibility of desired measurements over all participant testing. Another limitation of these methods with respect to capturing a musician's performance, is that to the restriction of movement. Attachment of any form of device to a performer may obstruct or alter a musician's natural movement. This could comprise the accuracy of measurements recorded. In 
image motion capture, occlusion of markers can also comprise the accuracy of measurements. Occlusion may occur due to the position of markers or the movement of the hand and fingers during performance. Variation in movement may block a marker during data collection, which would result in a missed trace from the specific marker. For EMG measurements, there is the additional disadvantage of the processing of noisy signals which can lead to inaccuracy in data analysis and difficulties in extracting significant measurements.

During a keystroke, a subject might involve their entire body in the motion. The goal of the current research was to analyze the interaction of the subject and key during a keystroke. Therefore, it was determined that it would be most effective to look at the hand, more specifically, a finger during touch. An option within this focus could be to focus on the fingernail of a subject. Image capture of the colour variation of a fingernail could be used to indirectly measure force application. However, this optical approach could hold high variability due to the surrounding room environment and the subject themselves. Additionally, in the future this method could be further difficult to integrate into multiple keys.

Overall, these methods each fail the criteria to implement a design which allows discrete data collection. All methods proposed would require the awareness of the participant. This could affect data collection in terms of authenticity of playing. A subject may consciously or sub-consciously alter their performance due to the presence of a research set-up on, or around them, during subject testing. The high variability between participants could also result in difficulties in collecting accurate data across participants in a user-friendly method. 


\subsubsection{Optical Measurement Approach}

The intricate movement of the piano action has led past researchers to assess the user-key interaction from an optical approach. Measurement of key and piano action motion have been achieved through various methods. One method has been that of optical interference measurement. The use of photodetectors allows collection of position and motion data through beam interruption, as the motion of the key would interrupt the beam. Work previously described by Askenfelt implemented such methods to monitor both key and hammer movement with photodetectors to assess key velocity and position [19]. Their work used these variables to indirectly measure the normal force applied to the key. Interference measurement methods are also implemented in MIDI data collection, which is also able to collect velocity data. These studies are developed on the assumption that the velocity data provided by MIDI is an indirect measurement of the applied force. However, the recorded velocity only captures a component of the key movement profile during key depression. Previous studies have been noted to use MIDI data collection in conjunction with additional measurement methods [31,54, for example].

The use of photodetectors to complete indirect force measurement can be inaccurate. Variability in playing and tempo would result in the method only being practical in controlled testing conditions. Furthermore, the calibration method presented in previous studies required participants to play at targeted force increments repeatedly, to detect the resultant velocity. These methods of user calibration carry error in: the inability of a user to repeat target keystrokes repeatedly, the need to calibrate for each user, and also possible error if participants are unable to replicate their natural performance movements. 
Another optical approach noted was the use of laser displacement measurement [59]. This method has also been adapted to collect the variables of position and velocity. These methods have more commonly been used to assess the motion of the hammer action and string contact. A review of these previous approaches have identified drawbacks to an optical measurement method. One disadvantage is that the accuracy of recording is dependent on the accuracy in positioning of the sensors. Photodetectors must be placed close to the key or action to ensure proper measurement. As a result in the positioning, an issue of discreteness and unobtrusiveness to participants may also be present.

\subsubsection{Acoustic Measurement Approach}

In researching quantitative measurement of performance, it was common to see acoustic measurement methods utilized [42, 43, for example]. Assessment of noise level has provided indirect analysis of overall force during playing. Calibration with noise level would again provide unique challenges. The method could be used to indirectly assess the overall force in playing under specific playing tasks. These would be limited to single keypress testing. Calibration through an acoustic method would not be accurate as it would need to be completed across each participant, and with the consideration of the external environment. This could greatly increase error. Instrumentation used to collect the response of the keys, could also complicate the analysis due to error between the perceived and actual response of the piano.

\subsubsection{Mechanical Approach}

The majority of studies reviewed in Chapter 2, utilized a mechanical-based approach. These methods entail the use of a force or position sensor to directly interact with the user or a piano key to collect measurements. Popular instrumentation used 
from previous approaches include force sensing resistors, force transducers, and accelerometers. Each of these options present different challenges in this application.

The completed review of literature noted that early research relied heavily on the use of force sensing resistors [39,50, for example]. Although validation of these earlier methods could provide new insight to the field, further research on this sensor concluded they do not meet the criteria necessary to complete effective data collection. Force sensing resistors (FSRs) are sensitive to humidity and shear force application. For this application, it means their calibration would be unreliable. Changes to the external testing environment could have a negative impact on the ability to accurately collect force readings. Similarly, they offer low repeatability as drift is often present in the system, which again would negatively impact the accuracy of calibration and therefore final readings. FSR would provide information on the normal force applied to the surface of the sensor, but would be unable to distinguish the true force vector and position of touch. Referring back to the earlier work from Parlitz [39], a sensor matrix foil was placed under multiple keys. This approach required a full modification to the piano. Additionally, this method would not provide a full touch profile during a keypress. Their design was limited to only collecting touch data when the key reaches the key bed. In reference to the sound production, this would align with the point after the hammer has lost contact with the repetition lever, and therefore no impact on sound production would be possible.

Force transducers have been applied both externally and internally in a piano key. Methods which placed a transducer atop a piano key are not only visible to the user, but may be physically obtrusive and impact a pianist's playing ability [31,42,48,49, for example]. A detectable, and possibly obtrusive, design may not provide an accurate representation of a true playing environment. Although methods in which a sensor 
is placed internally avoid the former issues, they do bring about new challenges. Past literature looked to measure force with the use of uniaxial force transducers, meaning the normal force to the sensor was measured. However, it can be argued that oversimplifications were made. These methods consider only one force component and one direction component for measurement [40,52, for example]. This assumption directly conflicts with keypress mechanics. Different finger postures, which vary the force vector angle applied by the finger, are present during playing. This would mean that more than one direction of force measurement would be required to provide a full picture on the finger-key interaction.

Accelerometers have been applied to complete measurements in relation to the key or piano action movement. These are a standard in mechanical sensors, as they are able to collect continuous acceleration data during testing. They have been used extensively in hammer shank acceleration research [18,19, for example]. Accelerometers could be affixed to a piano key to indirectly collect desired variables. However, data processing would not be straightforward. Acceleration data would need to be integrated to get velocity and position. However, as previous methods have also noted, complex calibration may result in an inaccurate system.

In the application of pianists, the option of a mechanical approach is advantageous to collect force and position information. All of these methods would need to consider that mounting of sensors to a key or action would add mass to the system. This may impact key movement, and the pianist's ability to replicate their performance during testing. To achieve the measurements discretely, sensors may need to be permanently secured to a piano key, or placed within the key. Pianos are an expensive instrument. Therefore, instead of developing an entire piano with embedded sensing instrumentation, a more adaptable and cost-efficient design was applied. This would 
be possible through re-development of the piano key being used for testing with the manufacturing of a replica key. The sensing instrumented key could then be integrated into keyboard testing. This would allow data collection to take place on pre-determined keys during playing tasks. This discrete design approach could be achieved through two main methods.

\section{Develop a Key-Specific Sensor}

A key-specific sensor could be designed to collect the desired force and position profiles during a keystroke. This custom sensor could then be embedded in a replica piano key to complete testing. To properly develop a sensor to measure the desired variables, an understanding of the theoretical motion and force application on the key would be required. Through knowledge of the key motion, the force applied by the finger to the piano key could result in bending, torsional, or axial loading cases on the key, dependent on the angle and location of force application.

Strain gauges could be implemented, as they can measure small deflections experienced by a key during a keystroke. The use of strain gauges can be used to measure deflection and can be arranged in various ways to allow the deflection in more than one axis to be detected. As strain gauges are designed to be responsive in one direction, this allows differentiation between different directions of loading. Although the gauges are used to measure strain, there is a direct relationship between strain and force which can be applied, [60,61, for further reading and basic strain gauge theory]. To achieve the desired force and position information, an original design would need to be developed to ensure all variables are accounted for. There are two main ways this could be achieved- with commercial sensors or a custom design. Selection of this method would require calibration of the designed sensor. The application of advanced multivariate calibration approaches is beyond the current scope of this initial 
work. This research would aim to validate that data collection is possible through this method.

\section{Parametrized Design}

An option in gauge placement would be to develop a parametrized design. This would require gauges to be precisely placed along the required axes to measure strain. For this application, there is interest in collecting force data in the 3-axes as well as finger position in 2-axes along the keytop. Therefore, one gauge could be placed along the axis of interest for each force direction. To further measure position, an additional gauge would need to be placed along that respective axis. This would provide comparison of the results at the two points along the defined measurement section. With this in mind, for a parametrized design for keystroke measurement a total of five gauges would be required.

A challenge with this method would be the required accuracy in gauge placement. Gauges would need to be placed precisely in order to take accurate measurements, including positioning. If a gauge were to be placed off-axis, it would not properly detect the loading cases and provide an inaccurate force profile. Verification of this method would further require an assessment of the data collected under the five different applied loads, to determine the expected response of each strain gauge. Once verified, this method could be used for accurate measurement, with a discrete method. This design would provide simplicity in data analysis, as fewer signals would need to be analyzed, and the response type of each channel would be known. Strain gauge selection would dictate the repeatability and sensitivity of the system. A drawback of this method would include the overall repeatability of the design. As this design involves precise hand placement of strain gauges, the ability to accurately repeat the design across more than one key is difficult. Therefore, although this method could 
prove sufficient for one key, it may not be effective in reproducing the sensor across multiple keys.

\section{Over-parametrized Design}

Another option with the use of gauges would be to create an over-parametrized design. Through this design approach, the number of gauges placed would be greater than the number of variables of interest in recording. In this application, this would result in more than five strain gauges being used. The benefit of this method would be that less precision is necessary in the placement of the gauges. An overparametrization would result in a redundancy in data collection; multiple gauges would measure the same input variable. This would increase the reliability of the system as more than one gauge would be detecting the applied force along an axis.

Although this method increases reliability and does not require as precise of placement, it would still present challenges. The gauge assembly would be simplified, howeveer a more involved multi-variable data assessment would be required. Through this method, the channel response of each gauge to an applied force may not be theoretically known, as the precise placement of a gauge along an axis would not be a requirement. Therefore, various loading conditions would need to be applied to collect response data from multiple gauges. These responses could then be assessed to determine the overall system to provide the force and position data. Issues again could arise in reproducing the design across multiple keys. As gauge placement is less restricted, the main concern would lie in differentiating the responses from each channel with respect to different loading conditions. A unique testing and data analysis method would be required for each developed sensor. 


\section{Develop a Key with Commercial Sensing Equipment}

Rather than implementing gauges to create a custom sensor, commercial sensors could be acquired. These could be integrated into a re-developed key, or fixed atop a key, to collect force and position data. To record these variables, various sensing options could be used.

\section{Force Sensors}

Commerical force sensors can vary greatly depending on their intended use. For example, bending beam load cells, s-beam load cells, and button load cells all perform the function of force measurement, but designed for different loading cases and design specifications. Previous literature relied on the use of button load cells [62, for example]. Button load cells, commonly referred to as compression cells, are sensitive to normal force application. Multi-axis sensors can also be sourced commercially. One drawback of this instrumentation, is lack of customization. One or more sensors would need to be sourced which meet all of the design and measurement specifications for this application. Additionally, although instrument specifications would be provided, the calibration and use of such devices would carry out the measurement and data collection in a process that is essentially unknown. This form of "black box testing", although at times effective in their measurement, could provide additional challenges. If the system is not behaving as expected, adaptation and verification testing with the commercial equipment could lead to an ineffective system. The troubleshooting and adaptation of a commercial sensor may pose to be more difficult. As the sensor would be integrated into the prototype key system, potential errors from keystroke measurement and force component differentiation could negatively impact the ability to collect useful data. 


\section{Contact Position Sensors}

Position of touch is a desired input, which had been overlooked in previous studies. Measurement of contact position could be achieved through a multi-axis force sensor, or the integration of multiple force sensors. However, capacitive touch sensors could also be implemented to measure contact position. Similar to the touch screen technology on a smart device, capacitive touch sensors could be used along the top of a key to define the contact position as well as contact area of touch. Previous works by McPherson [56, for further reference] was able to collect position, contact area, and duration of touch, through the use of their developed capacitive sensors, TouchKeys. The main drawback of this approach was noted as the aesthetic issue of a sensor being placed along the key surface. Not only could this interfere with the musician's ability to perform, it could also impact the movement of the key due to the added mass and height of the key, due to a sensor affixed atop of the key top.

\subsubsection{Conclusions}

It was clear that each approach carries disadvantages. For this research, the elements of unobtrusiveness to participants and accurate force and position measurement were critical. Research in the fields of sports biomechanics, computer keyboard ergonomics and assessment, and touchpad development were also reviewed. These fields appeared to use similar methods as those previously applied in musician tracking. Therefore, through the review of previous research and the main approaches applied, it was confirmed that a mechanical approach would provide the most ideal approach. A mechanical approach would require the purchase, or design, of a sensor, as well as the possible development of a replica piano key to allow instrumentation integration. After the thorough review of previous works, it was concluded that to develop a final conceptual model for this application, an outline of the quantitative 
measurement specifications were required.

\subsection{Measurement Specifications}

In developing the overall project goals, it was first necessary to determine the parameters of what was required to be measured. It was critical to meet with current music students and teachers to further discuss design inputs. Consultations provided similar expectations as those evaluated by the researchers. Their interests of evaluation included: assessment of variance across musician's performance and rehabilitation methods, evaluation of more than one note at a time, assessment in keystroke variances between injured and healthy musicians, comparison of force between lefthanded and right-handed playing, collection of force changes throughout an entire keystroke, as well as contact profiles. These criteria were translated into a set of measurement requirements.

To summarize, the intent was to develop a measurement method which could meet the following requirements:

- force measurement of all components of the applied contact force

- contact position measurement

- not require permanent modification to a piano

- unobtrusive to a pianist's natural performance

- undetectable to a pianist, to ensure natural performance is not altered

- reproducible for future implementation with multiple keys

- user-friendly to simplify testing and setup across participants 
With the conceptual outcomes defined, the thesis design requirements could be developed. At this stage in research, it was noted that not all requirements would be met fully. As previously stated, the contribution of this thesis is the hardware design, therefore the main focus was on the first five requirements. However, it was important to outline the user design inputs for the project to develop the quantitative requirements of the finalized design.

\subsubsection{Key Physical Parameters}

Development of a replica key to allow integration of sensing instruments would require careful consideration. For the purpose of initial design and validation testing at this phase of research, replication was completed with an upright Yamaha Disklavier MX1Z piano key. Although this phase of research has focussed on an upright piano key, upright and grand piano keys provided many similarities that allow this design to be universal. The remainder of the research will use the physical parameters and geometric dimensions in the model from the specified key. In terms of key length, medium sized grand pianos and upright pianos are generally the same length. The structure of a piano, in regards to key placement and rotation across a key pin, remains the same with piano configurations [1].

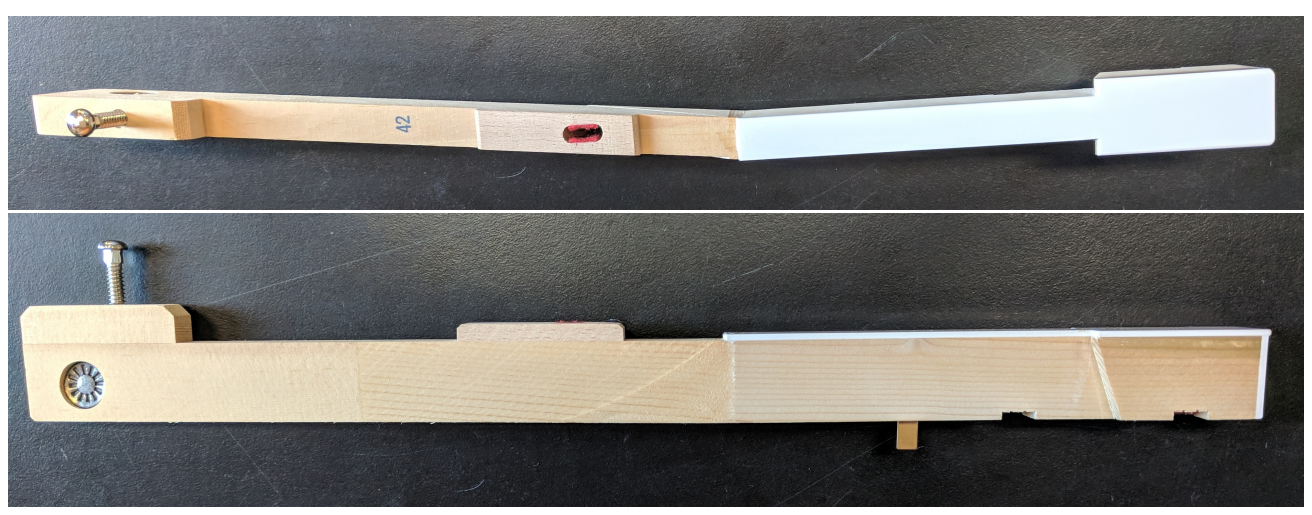

Figure 3.1: Key 42 Selected for Initial Design and Testing Implementation 
To complete the instrumented key, the specific key to replicate had to be selected. After consultation with prospective users, it was identified that a key that occurs in the middle of common musical phrasing would be beneficial in data collection. Furthermore, a key in which finger patterns do not commonly require use of the thumb, in the tasks typically used in experiments, would be beneficial for future work. With these recommendations in mind, the D above Middle C, key 42, was selected to model, see Figure 3.1. To ensure a pianist's natural performance was not altered during testing, piano key properties need to be maintained. The following piano key properties were necessary to create a dynamically-equivalent piano key:

- key geometry

- key surface appearance and material

- key mass properties

An instrumented key must replicate the original key geometry to allow full testing in a piano. This was necessary to allow an undetectable key to a user as well as a key that could function in the larger system of the keyboard and piano action. Simply put, the replica key must be identical in external geometry including, total length, width, and height, position of balance pin holes, and position of the piano action contact point. To achieve the specific specifications of the size constraints, measurements of the piano key were completed. A 3D scan of the piano key was completed with an Artec Space Spider 3D scanner. Further details on this process, and the detailed geometry of the key can be found in Appendix A.

Additionally, to ensure the pianist's natural performance is not altered during testing, maintaining the key surface appearance and material was vital. To meet this requirement, it was determined that a plastic key top could be obtained to place over 
the replica instrumented key. As outlined in Section 2.1, the key front and key top are the only visible portions of the piano key. Therefore, material selection for the remainder of the key was not critical in reference to appearance. This was additionally true for any sensing instrumentation.

To ensure uniformity of key movement across the replica and adjacent keys in the keyboard, it was important to maintain the mass properties of the key. These properties include the total mass, centre of mass, and mass moment of inertia of the piano key. Variation of mass across keys within a keyboard or against different piano models is present. Therefore, the properties of the specific key for the proposed model were used to develop the mass properties. The centre of mass of an object is loosely defined as the position of balance across an object. This refers to the position in which the moments from the mass elements from an object sum to zero. Due to the intricate shape, and multiple materials used to manufacture a key, the centre of mass was found experimentally. The mass moment of inertia, is a quantity which expresses the resistance of a body to angular acceleration, about a specified axis [63]. This value was also determined experimentally. These methods, are further outlined in Appendix A. These measurements identified the mass of the key as $81.41 \mathrm{~g}$, the centre of mass, measured from the front edge of the key, as $(0.02 \mathrm{~m}, 0.154 \mathrm{~m}, 0.013$ $\mathrm{m})$, and the mass moment of inertia as $1.38 \times 10^{-3} \mathrm{~kg} \cdot \mathrm{m}^{2}$ and $1.40 \times 10^{-3} \mathrm{~kg} \cdot \mathrm{m}^{2}$ for $I_{z}$ and $I_{y}$, where $z$ is the vertical direction from the key and $y$ is the lengthwise direction along the key.

These physical design specifications would be critical to ensure the design remained unobtrusive. A sensor system should not interfere with a musician's movements. This includes alterations that may alter movement of the key which could impact playing as it may require altered movements. Any perceived changes in the key could lead to 
changes in performance which would result in testing conditions not matching a true playing environment.

\subsubsection{Sensor Parameters}

Section 3.1 provided insight to the previous attempts of key force and movement measurement. A clear void in the previous research has been the ability to integrate a sensor system into a piano key, to complete testing without modification to the piano or a pianist's playing. This new step in the area of research required strict sensor parameters to be defined.

\section{Physical Parameters}

As the intent of the research was to integrate the sensing system into an instrumented key, it was necessary to set physical parameters for the measurement instruments used.

\section{Geometry}

The geometry of the key was used as the size restrictions for the sensor. As it was of interest to asses key touch at the front of the key, these dimensions are outlined below in Figure 3.2. It should be noted that the dimensions were recorded in inches. The final design was drafted in these units as the machine shop, which completed the manufacturing of the key body, preferred imperial units for design submissions. These restricting dimensions were selected as accuracy in measurement could be achieved through sensing instrumentation being integrated at the front half of the key, before the balance rail. A secondary parameter was set if commercial devices or the fabricated design were unable to fit these size constraints. It was determined that if designs were unable to fit these parameters, the option of including more than one 
key into the design would be possible. The adjacent keys were included as a design option to house necessary wiring in the case that the first geometry parameter could not be met. All sensors and designs assessed were referenced to original key geometry to ensure the sensor(s) would meet the size constraints.

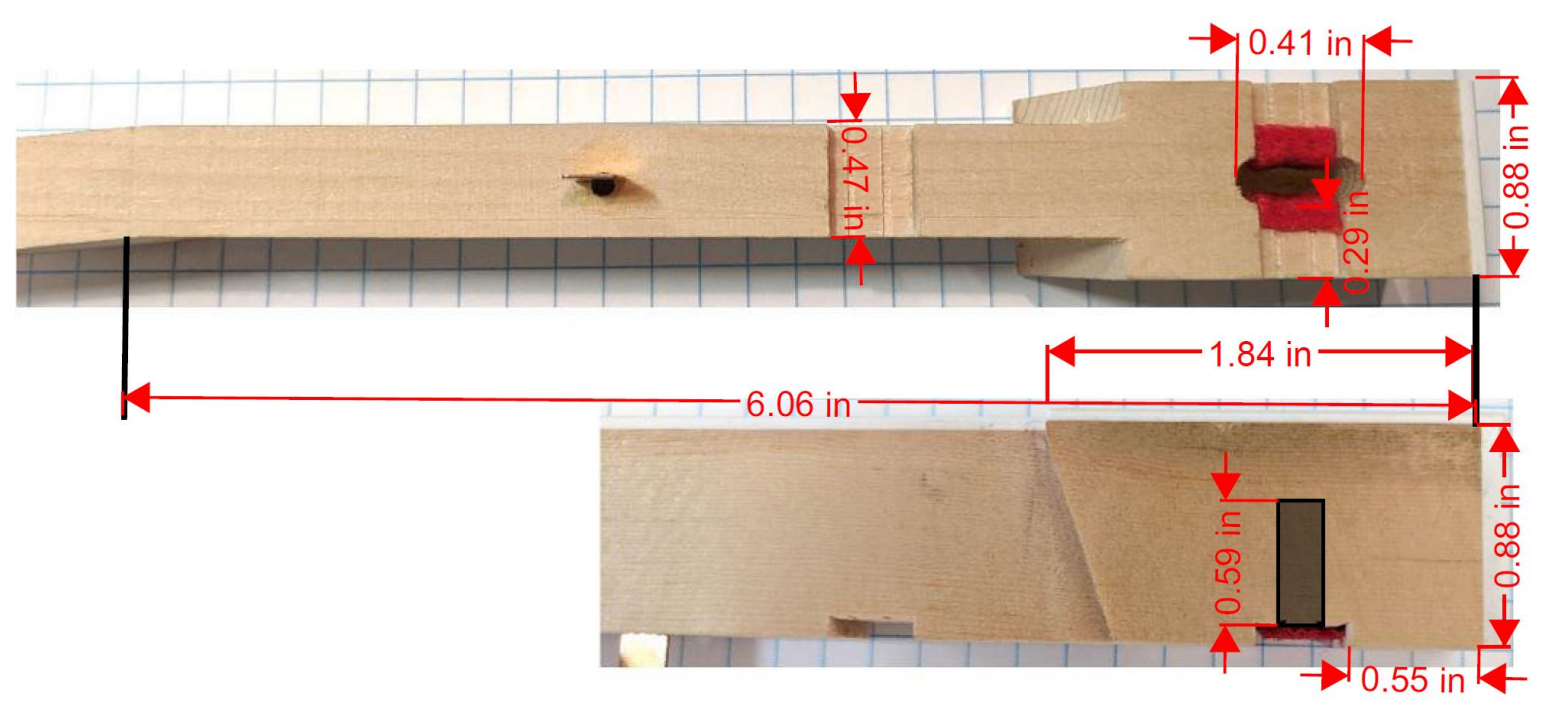

Figure 3.2: Restricting Dimensions of the Key Front (Unique to Key 42 of the Yamaha Disklavier MX1Z), with the inclusion of the front balance rail pin height

\section{Weight}

In setting weight specifications for the sensing instrumentation, it was noted that a lightweight sensor or system would be necessary. At the time of developing specifications, it was not yet clear what the exact weight parameter of the sensing system would be. The material and design of the replica instrumented key would greatly impact the allowable weight of equipment. The user sensitivity to an alteration in the overall mass of the key in comparison with the original was also unknown. This question was later investigated in Chapter 4. The lack of specificity in the early stage of design was not noted as a concern as available sensing options which met the 
geometry parameters would likely withhold the lightweight requirements.

\section{Measurement Parameters}

\section{Measurement Range}

The measurement range of a sensor defines the minimum and maximum detectable values. To develop a measurement range, previous works were reviewed to evaluate the force ranges detected during testing. As previously noted, earlier studies only took into consideration the normal force applied to the key.

Previous studies failed to properly select a sensor that could capture both the minimum and maximum forces that could be applied to a piano key. To define the minimum detectable force, a review of piano calibration was completed. It was noted that a piano key has a calibrated down weight. Piano key down weight is a term used to define the minimum weight required to depress the end of the piano key. It is referred to as the down "weight" as it is evaluated by placing calibrated weights on the end of the key. The down weight of a piano key normally ranges between 50 to 60 grams, which is standardized across both grand and upright pianos [1]. Using the lower limit of $50 \mathrm{~g}$ the minimum detectable force of the sensor was set to $0.5 \mathrm{~N}$.

To determine the maximum force that may be detected, a review of previous literature was completed. The early works of Parlitz only recorded forces below $20 \mathrm{~N}$ from tied finger tests which asked for subjects to play with considerable loudness [39]. This contradicted studies by Askenfelt and Jansson, [19], and more recent work by Kinoshita et al., [42] which utilized force transducers. The former research recorded forces of $8 \mathrm{~N}$ when subjects were asked to play at a piano dynamic level and $50 \mathrm{~N}$ when asked to play at fortissimo. The latter paper found a wider range of results with forces of $3 \mathrm{~N}$ when asked to play pianissimo and $60 \mathrm{~N}$ or higher when asked to 
play fortissimo. In review of the minimum values, it was noted that they may not be accurate as many studies failed to consider the possible minimum force application, which resulted in readings being limited to the equipment's measurement range. However, additional studies did fall within the average maximum values. As variance is expected in testing across different musical instruments and musicians, the range boundaries defined should be used as guidelines. An approximate maximum normal force application was therefore set as $60 \mathrm{~N}$.

A requirement of the sensing system is to record forces both normal and parallel to the surface of the key. Previous publications did not provide measurements of these values. However, from an assessment of common piano hand and finger postures, it can be assumed that the force parallel to the key will only be a fraction of that experienced from the normal force. The force range of parallel forces were considered from $0 \mathrm{~N}$ to $25 \mathrm{~N}$. The maximum force was developed from an assumption of the applied force being a function of the maximum normal force with the consideration of the frictional force developed between the finger and key surface. The average coefficient of friction of approximately 0.4 was used in this application [64].

\section{Maximum Overload Capacity}

In relation to the measurement range is the maximum overload capacity. With the low measurement range set, understanding of the force which can provide permanent damage to the sensing system was important not only in selection but also design of the sensing system. As the instrumented key would be around various subjects in an open environment for testing, there was a possibility that an interaction could result in an undesired force application on the key. 
An estimate through anthropometric tables would suggest a static force if a forearm and hand were applied to the key to produce a force of $20 \mathrm{~N}$ which is well within the measurement range selected. If a subject were to lean on the keyboard, with an estimate of $25 \%$ of their body weight, the applied force could increase to $220 \mathrm{~N}$. Furthermore, if an object were dropped on the key, the applied impact force could reach these ranges. This estimate is outside of the measurement range previously set. Therefore, an approach could be made to set the overload capacity to consider this upper range, or to have the key designed to fail before this load was reached to avoid damage to the instrumentation. As the estimate of applied body weight was a high-range approximation, it was not deemed critical for the sensor to have an overload capacity in this range. Instead, a safety factor was applied to consider the overload. A safety factor application of 2 was applied. Therefore, the maximum overload capacity was $120 \mathrm{~N}$, for the maximum applied force being the normal force.

\section{Resolution}

Resolution is the smallest increment in measurement a sensor can detect. Variation in force during the full depression of a keystroke could vary considerably. In addition, these slight variations in force application could be of interest in future research. Therefore, a small resolution would be required of a sensor to ensure sensitive readings which could detect the small load changes. A sensor's resolution should be considerably lower than the minimum variation in the force readings. The minimum force to be applied is $0.5 \mathrm{~N}$, but the variation once the key is struck could be much smaller. Few previous reports indicated the continuous force variation throughout a keystroke. It was determined that to ensure all significant variation is detected, a resolution of $0.01 \mathrm{~N}$, equivalent to a $1 \mathrm{~g}$ weight on the key, would be applied to the design specifications. It should be noted that the resolution would also be limited by 
the signal processing hardware selected.

\section{Accuracy}

Accuracy, in terms of design specifications, considers how well a sensor measures the inputs from the environment. In this application, accuracy is a measure of how well the sensor was able to return the correct measure of force and position. Consideration of accuracy is critical to completing useful data collection. As low forces will be recorded, a high accuracy will be required in the readings as a small deviation could make a significant difference in the reporting of the force applied. Previous publications that completed measurement within the defined range of force variation, used $0.5-1 \%$ as an appropriate accuracy for testing. As variation in forces in previous publications occurred within a small variance, this maximum range value is justified. Due to the lack of previous measurement methods which considered shear effects, previous data collection results are unable to be compared. Therefore an approach to achieve the highest accuracy possible was developed. This is viewed in aspirational goal to achieve an error percentage of $1 \%$.

\section{Reproducibility}

Reproducibility considers the ability of a sensor to produce the same measurement result when external conditions have been altered. It was of interest to complete testing across multiple participants, with future research spanning across various testing spaces. With this in mind, it was important that the system was able to repeat consistent measurements across multiple trials, including variance in room conditions. Although testing was to be completed within well regulated spaces for musical instru-

ments, it was important when reviewing sensing options to consider their performance in different temperature and humidity environments. 


\section{Signal Processing Parameters}

\section{Sampling Frequency}

A keystroke, depending on repertoire tempo, can last a short period of time. Previous publications $[39,42]$ recorded a high range of force variation over a small time range of 25-100 ms. To ensure sampling captures the initial key depression, a high number of samples should be acquired. In assuming a keystroke can occur over 50 ms, it would be acceptable to take at least 50-100 samples over that time period to achieve a response throughout the force profile. This would resolve a frequency of at least 1000-2000 Hz. This is the baseline required for the sensing system. Anything below this parameter will not produce sufficient data, as pieces of the key force profile may be lost.

\section{Noise}

The instrument noise of the system limits the force measurements recorded. A highsignal-to-noise ratio is required to ensure the small force variations are recorded and distinguishable from any noise. Further filtering may be necessary to meet a low-noise requirement for data analysis. It is also possible that wire movement and adjacent key movements could be recorded by the sensing system, and contribute to electrical noise of the system. A low-pass filter may be beneficial in future stages. With a set resolution of $0.01 \mathrm{~N}$ a baseline noise maximum of $0.005 \mathrm{~N}$ would be acceptable.

\section{Specifications Summary}

The quantitative design inputs are specified in Table 3.1, below. These key and sensor specifications were used as the framework to develop the sensing instrumentation. The input specifications were later compared in Section 7.4 to the final design 
outputs.

Table 3.1: Design Input Specifications

\begin{tabular}{|l|l|l|}
\hline Metric & Units & Target Value \\
\hline \hline Final Key Mass & $g$ & 81.41 \\
\hline Centre of Mass & $m$ & $(0.02,0.154,0.013)$ \\
\hline Mass Moment of Inertia, $I_{z}$ & $\mathrm{~kg} \cdot \mathrm{m}^{2}$ & $1.38 \times 10^{-3}$ \\
\hline Mass Moment of Inertia, $I_{y}$ & $\mathrm{~kg} \cdot \mathrm{m}^{2}$ & $1.40 \times 10^{-3}$ \\
\hline $\begin{array}{l}\text { Force Measurement } \\
\text { (normal to key surface) }\end{array}$ & $\mathrm{N}$ & $0.5-60$ \\
\hline $\begin{array}{l}\text { Maximum Overload Capacity } \\
\text { (normal to key surface) }\end{array}$ & $\mathrm{N}$ & 120 \\
\hline $\begin{array}{l}\text { Force Measurement } \\
\text { (parallel to key surface) }\end{array}$ & $\mathrm{N}$ & $0-25$ \\
\hline $\begin{array}{l}\text { Maximum Overload Capacity } \\
\text { (parallel to key surface) }\end{array}$ & $\mathrm{N}$ & 50 \\
\hline Resolution & $\mathrm{N}$ & 0.01 \\
\hline Accuracy & $\%$ error & 1 \\
\hline Sampling Frequency & Hz & 1000 minimum \\
\hline
\end{tabular}

\subsection{Finalized Conceptual Design}

With the measurement requirements outlined, the two mechanical design avenues were re-reviewed. To complete this, a review of commercially-available sensors was made. Surveys of sensors used in previous research as well as available instrumentation from manufactures were evaluated. Although different measurement methods were considered, the summarized review highlights the findings of the force transducer search. An overview of this search is available in Appendix B. Availability of 6-axis, 3-axis, and 1-axis sensors was considered. This search included both capacitive 
and piezoelectric force transducers. As force transducers span a wide range of physical parameters for various testing applications, miniature and sub-miniature sensors were reviewed. It was quickly determined that the geometry constraints would be a limiting factor in sensor selection.

A 6-axis sensor would accomplish all desired force and position measurements. After eliminating sensors which did not meet the geometry and force range constraints, two 6-axis sensors remained for further review. One of such sensors was an ATI Industrial Automation Nano1\%. This sensor was commercially available, and initially appeared to meet the physical and measurement specifications. The lightweight design met the measurement range, accuracy, and resolution requirements. The body of the sensor could be integrated in the front of the key, while remaining within the size constraints. However, the sensor would require wiring from the body of the sensor to the data acquisition system. The available wire output configurations would not allow the sensor to be integrated in the key, within the configurations that fit the size constraints. Additionally, this option did not meet the criteria of reproducibility. A sub-miniature 6-axis sensor was not cost effective to implement in more than one key in future research. Overall, these results led to further review of alternative instrumentation options.

The option of using multiple 3 -axis or 1-axis sensors was also reviewed across various manufacturers. Weight and size constraints were again used to first filter the results, with approximately 50 sensors surveyed further. Models such as the Honeywell Model 13 and the Omega LC300 Series sensors were assessed. These models had been used in previous piano research studies in which the sensor sat above the key top. As more than one sensor would need to be integrated internally, the main issue again was the size constraint of multiple sensors, their wiring systems, and the necessary 
house to ensure the configuration of the sensors provided the correct axis of force measurement. The additional complication at the key front was the necessity of the front balance pin to pass through the centre of the front, which could also not be obstructed by wiring.

With respect to force measurement range, the majority of the 3- and 1-axis sensors were able to measure up to $200 \mathrm{~N}$ of force. However, with the lower degrees of freedom, the sensitivity to shear loading was considered. In assessing uni-axis load cells, such as compression load cells, the impact of off-axis loading on the sensing equipment was evaluated. It was noted that off-axis loading would not only have a negative impact on the accuracy of the readings, but it could cause permanent damage to a load cell. Integration designs would need to carefully assess loading conditions and ensure only one axis of loading was applied for each cell configuration.

Strain gauges were also surveyed to confirm size and measurement specifications. It was clear that a variety of options were available that met both the physical and measurement specifications. Additionally, different configurations were available to allow multiple axes of sensing in a small testing space.

\subsubsection{Sensing}

Design specifications were compared with the available commercial equipment. It was determined that with the unique physical constraints of the key, a commercial multi-axis sensor approach was not possible. Furthermore, a configuration of multiple compression sensors to test loading in more than one axis was not favourable. Offaxis loading to the sensors would result in damage to the device, which would impact its ability to take measurements. The configuration of multiple commercial compression sensors could also carry significant error. Although this approach would provide 
commercially-manufactured sensors, and therefore a lower error in fabrication, this would be a "blackbox" approach that would be difficult to adapt to this specific problem. The instrumentation and signal manipulation that are integral to commercial sensors may limit later applications of multivariable calibration techniques. Additionally, sensors within the desired measurement range, would pose an intricate configuration option when considering wiring the system. Therefore, it was determined that developing a key-specific sensor, based on strain gauges, would provide a more efficient key design, as well as less restriction in obtaining all design specifications. The use of redundancy in gauge placement was further evaluated through a theoretical analysis in Chapter 5.

\subsubsection{Geometry}

A key-specific sensor provides flexibility in the overall geometry of the sensing instrumentation. It was clear that a model would allow integration of all sensing equipment inside of the key. However, further details on the overall design were dependent on the factors of materials used and their impact on the changes to the mass properties of the system. Therefore, further consideration on the impact of the integrated design and the overall modelling of a replica key were completed. 


\section{Chapter 4}

\section{Impact of Mechanical Piano Key Alterations on Musician Performance}

Previous studies have completed user testing which has required modifications of a piano key and action due to the affixing of various measurement instruments. However, focus has not been made on the impact an increase in mass may have on the key movement and perception from a pianist. Early development of design approaches were discussed with current piano students and teachers. A concern voiced by this focus group was that any mechanical changes to a key would have an impact on the keyboard touch and response.

Continued understanding of pianist touch has previously assessed pianist kinaestheic memory. Participants completed touch tasks to reach a target force under varying feedback conditions [62,65,66, for example]. These forms of studies identified that pianists are sensitive to both audio and tactile feedback during playing tasks. Studies have also been completed to gauge pianist perception to vibration caused by the strings in a piano. Experimental testing with participants identified vibrations at the keys is fairly weak, to a point in which they are not detectable. Even at the loudest dynamic level, it was noticed that at the keys the vibration barely rose above 
the detection threshold around $200 \mathrm{~Hz}$. However, assessment of the regulation of the weight of the keys or the impact of weight distribution across points of the key has not been studied [67].

Pianists are comfortable playing with consistent gradual variation in the physical parameters of piano keys that is normally present across a keyboard, due to variations based on pitch (e.g. string length). From the perspective of piano key manufacturing, it is not the goal during the manufacturing process to produce all keys to be the same. Variation intentionally exists in the masses of the piano keys, and the range can be significant. Piano technicians regulate piano keys by adding masses, typically to the back end of the key, to regulate the keys across a keyboard. Limited previous research on pianist perception to varied degrees of touch has been completed. Research focussed on the correlation between weight discrimination and piano proficiency has been attempted by Ortmann in the 1920s, [68]. Their methodology utilized an experimental apparatus of two levers to simulate the movement of two piano keys. The levers were affixed with a sliding weight which was incrementally increased to determine the minimum detectable weight discrimination of each participant. They determined the average detectable increase to correspond with a $5.9 \%$ total weight change. Testing limited the participants to the type of touch, dynamics used during touch, and the finger used in the repetitive lever pressing task. More efforts to assess pianists have used generic somatosensory and motor function evaluation approaches, [69,70, for example]. Evaluations such as a 2-point discrimination test and finger-lifting weight discrimination tests unfortunately are not unique to pianists and the movement of the piano action.

As the average upright piano key mass is under $100 \mathrm{~g}$ [71], added instrumentation may have a significant impact on key touch and movement. From a perspective of 
the mass properties of the key, an alteration to the key may significantly alter the movement of the key. As previously outlined in Section 2.1, the piano key can be visualized as a lever which rotates about the balance rail during key depression. This rotation about the balance rail occurs if the force applied is larger than the regulated key down-weight. Adjustment of the mass at different positions of a piano key impacts the mass distribution which effectively alters the total mass, the position of the centre of mass, and the mass moment of inertia, as described in Section 3. While from the point of view of the moment of inertia, the addition of a mass introduces a dynamic

problem. However, the static mass and distribution is important to key return, with the static imbalance present across the keys.

\section{$4.1 \quad$ Objective}

It is clear that little research has been completed on user sensitivity to varied degrees of inconsistent physical variations in piano keys. Due to the sensitivity of the piano action it is critical to evaluate a pianist's perception to these changes and its potential impact on performance ability. Therefore, the objectives of this experiment were two-fold:

- Determine if, and to what degree, adjustments to the mass of individual piano keys are detectable.

- Determine if, and to what degree, adjustments to the centre of mass and moment of inertia of individual piano keys are detectable.

These objectives led to the development of an experimental study to determine the validity and scale of the concern of mechanical adjustments to the key and action. This study was approved by the Carleton University Research Ethics Board and 
informed consent was provided by each participant prior to testing. Ethics clearance can be viewed in Appendix C.

\subsection{Experimental Design}

Temporary alterations to a piano key and action are limited. It was concluded that two temporary alterations could be included in the study, being that of total mass increase of a piano key and key voicing ${ }^{1}$. Key alterations were developed over a 3-octave range from C3 to C6. Selection of the keys to be altered was considered over the fingering pattern of a scale and their position with respect to other altered keys. To avoid kinaesthetic after-images, in which impaired judgement of a key may be due to the influence of a previous key, the altered keys were spread out over the key range. A total of five keys were altered for participants to identify.

Key voicing hammer felt modification was applied to one key by a piano technician, to act as a test of touch perception being related to the tone outcome of the pitch. Four keys were additionally altered by increasing the total mass of the key. It was of interest to test the two possible cases that may occur through the manufacturing of a custom key; that of a heavier front-weighted or back-weighted key. These cases were translated to increasing the total mass by approximately $25 \%$ and applying that increase focussed to the front of the key ahead of the balance rail, or behind. To further test the variables of total mass, centre of mass, and mass moment of inertia two additional cases were introduced. The first case increased the total mass by approximately 50\%, balanced across the piano key. The second case increased the total mass by approximately $100 \%$ while maintaining the position of the masses balanced across the key. The order of the altered keys is shown below in Figure

\footnotetext{
${ }^{1}$ The process of a piano technician adjusting hammer felt density, which produces a brighter tone of the pitch [1].
} 
4.1. This experimental design allowed participants to play through the key range and evaluate if varying degrees of inconsistent key variations are detectable.

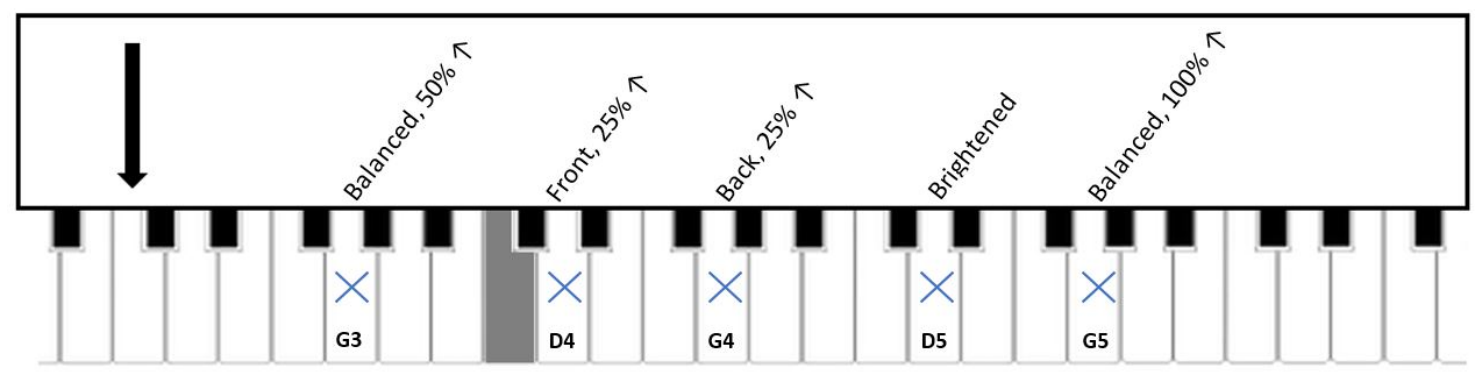

Figure 4.1: Outline of the Keys Adjusted in the Touch Perception Study with the 3-Octave Test Range Beginning on C3 (noted with the start arrow)

\subsubsection{Equipment}

The study was designed to implement non-permanent adjustments to the piano keys. An increase in piano key mass was achieved through placement of manufactured weights which were affixed to the predetermined position on the selected keys. The weights were designed to approximately match a $25 \%$ total weight increase in a piano key. This percent increase was approximate as the masses of the piano key vary, based on the piano design and the specific note. As the position of the weight varied from the back and front of the key, a keyboard cover was additionally placed to cover the black keys of the keyboard, to prevent participants from viewing the weights during experimentation. To allow easy application of the key weights, the fallboard of the piano was removed, and replaced with a temporary panel. These adjustments can be viewed in Figure 4.2.

The main evaluation method during the testing was the written participant responses. However, additional data were collected to allow for further analysis and 


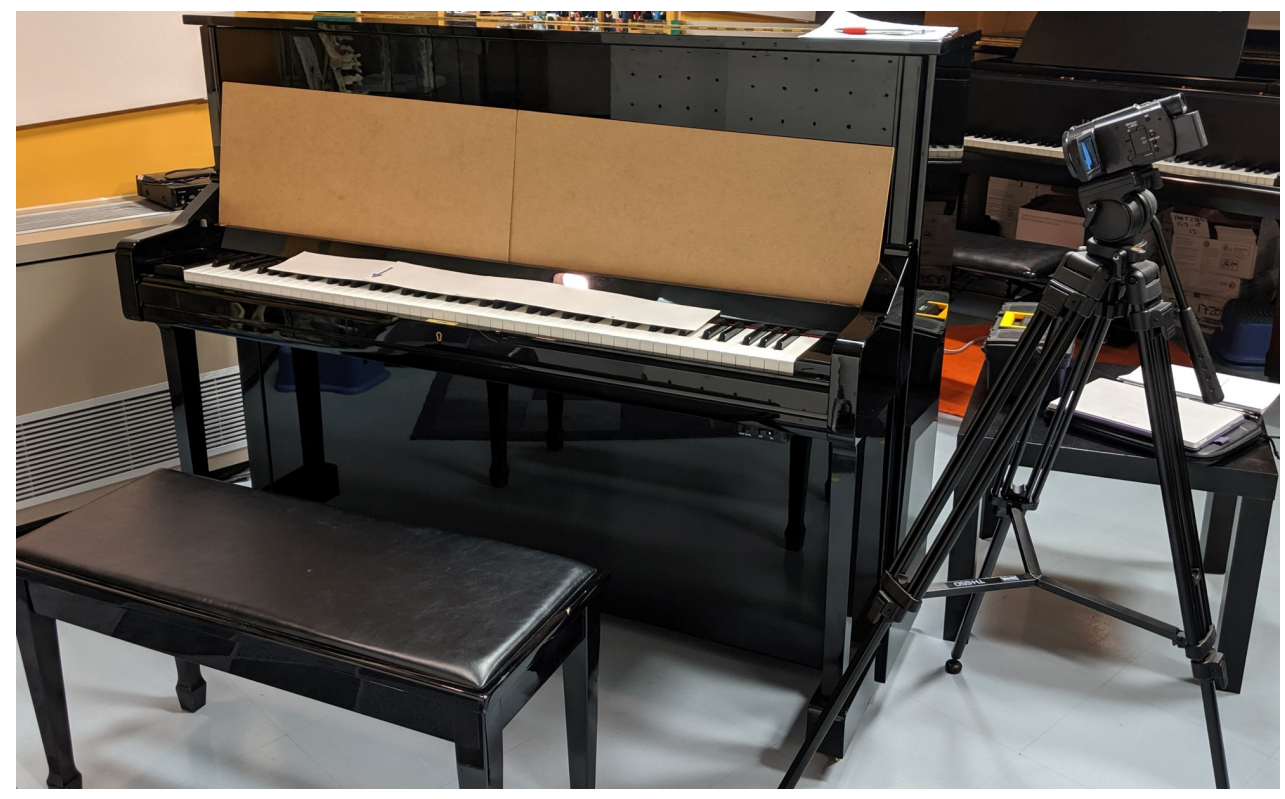

Figure 4.2: Overview of Experimental Study Setup

review of anomalies in the results. Additional measurements taken during the playing tasks consisted of video recording focussed on the participant's hands and MIDI recording which encompasses measurement of key velocity, pitch, and timing.

\subsubsection{Subject Recruitment}

Thirteen participants were recruited for the study. As the objective of this research was to evaluate pianist perception to varied degrees of change, it was necessary that participants had a pre-existing understanding of key response and down-weight of the keys. Therefore inclusion criteria were established to identify that participants must meet one or more of the following qualifications: have obtained their Grade 8 Piano $\mathrm{RCM}^{2}$ or equivalent, or are studying piano as their major instrument at a University level.

\footnotetext{
${ }^{2}$ Royal Conservatory of Music examination level. Grade 8 is the typical transitional level to that of an advanced student.
} 


\subsubsection{Task}

Participants were required to perform testing under two different conditions. The first condition was the one in which mass alterations and key voicing was applied to the key range. The second condition acted as a control in which no mass alterations were applied, but the brightened hammer was still present. The participants completed identical tasks under both testing cases and completed the two cases one after another. A control was included to evaluate the validity of the data collected from each participant. Throughout testing, participants were not limited to their dynamics, but were instructed to not use the piano pedals.

The first experimental task instructed participants to play an ascending and descending three-octave C major scale. Specifically, they were asked to only play with the right hand, begin on C3, repeat the scale three times continuously, and follow the suggested tempo. After completion of this task, participants were asked to indicate on their questionnaire any keys which they perceived to have been altered.

The second experimental task allowed participants to revisit the key range spanned during the 3-octave $\mathrm{C}$ major scale. However, during this testing the participants were instructed that they were free to play the notes in any preferred order, tempo, fingering, and dynamics. This allowed the participants to revisit any keys which they may have perceived to have been altered and further investigate them through varying their playing. Participants were not provided any restrictions on what they played, but they were notified when they had been playing for more than five minutes. After these tasks, participants were again asked to indicate their responses on a questionnaire. 
When the two experimental tasks under the first testing condition were completed participants were asked to sit to complete the final question of their questionnaire which was to draw final conclusions after completing the two exercises. At that time researchers altered the keyboard for the second testing condition. The two experimental tasks were then repeated under that condition.

\subsection{Results}

Questionnaires completed by participants provided a qualitative response from the experimental tests. In order to evaluate the data, the level of confidence in the responses were categorized. It was decided that the responses could be divided into three categories:

1. Identified with Confidence: The participant selected the key and was able to identify the applied change (ie. heavier, brighter)

2. Identified with Low Confidence: (one or more of the following)

- The participant selected the key but was unable to identify the nature of the applied change

- The participant selected the key but they were not confident a change was applied

- The participant selected a range of 3-5 keys which included an altered key

3. Did Not Identify: The participant was unable to identify the altered key

The division of these responses can be viewed in Table 4.1. The number of incorrect key selections were also recorded in order to further compare the participant responses. In the first testing condition, 5 participants identified unmodified keys. In total 8 
keys were selected, with 7 unique answers across the participants. This process was repeated in the control testing case. In this setting, it was found that a total of 9 participants identified unmodified keys. In total 28 keys were selected, with 19 unique keys answers. The conclusions of the unmodified key selection in both testing cases included that of the categories of identified with confidence and identified with low confidence. In the control case, 2 participants identified the brightened voice with confidence, 2 participants identified with low confidence, and 9 participants were unable to identify this change.

Table 4.1: Participant Responses Summarized of the Number of Subjects Who Identified an Applied Key-Alteration Test Case

\begin{tabular}{|c|c|c|c|c|c|}
\hline \multirow{2}{*}{ Participant Response Type } & \multicolumn{5}{|c|}{ Alteration Type } \\
\cline { 2 - 6 } & $\begin{array}{c}\text { 25\% Increase, } \\
\text { Front-Weighted }\end{array}$ & $\begin{array}{c}\text { 25\% Increase, } \\
\text { Back-Weighted }\end{array}$ & $\begin{array}{c}\text { 50\% Increase } \\
\text { Balanced }\end{array}$ & $\begin{array}{c}\text { 100\% Increase } \\
\text { Balanced }\end{array}$ & Brightened Voice \\
\hline \hline Identified with Confidence & 0 & 2 & 0 & 1 & 3 \\
\hline Identified with Low Confidence & 1 & 1 & 0 & 1 & 2 \\
\hline Did Not Identify & 12 & 10 & 13 & 11 & 8 \\
\hline
\end{tabular}

An overview of the results from this phase of testing provided interesting findings. Overall, key voicing alterations were noticeable to more participants than the mass modifications. These participants stated not only that the key was brightened but that they were extremely confident in their selection. This may suggest that confirmation of quality of tone holds a higher importance than the quality of touch. The perception to mass alterations were much lower than previous studies reported. This was expected as this study, unlike previous research methods, completed testing within the piano with varied mass distributions.

The participant group only showed confidence in identifying two of the mass altered keys, being that of the back-weighted key and the double-weighted balanced 
key. Confidence in these alterations were only noted by 3 participants (out of 13 participants). These participants additionally noted that scale testing did not provide confidence in identifying key changes, and further investigation of the key range was required. Between the two detected keys, the comparison between the detection rates of the back and front weighted keys may suggest that perception to change may be more sensitive to position of the applied mass in lower weight cases. When assessed by a piano technician, the ability to detect key changes was noted to be based on the key return speed, which is an effect of the static distribution of mass of the key. An interesting finding was the number of unmodified keys which were incorrectly identified as altered during testing.

As highlighted previously, the number of subjects who incorrectly selected an unmodified key greatly outweighed the number of participants who were able to correctly identify a key change. This was evident in the control testing case in which participants confidently selected a total of 28 keys as modified, although no alterations to the keys had been made. Out of the three octave range of the testing (a total of 22 notes), across the 13 participants, 28 keys were misidentified in the control testing. This is a stark difference to the modified key testing case. Out of the range of 22 notes, across the 13 participants, only 3 participants were able to detect the mass-altered keys with confidence. In comparing these results, it is evident that the misidentification of unmodified keys was quite significant. These results show that mass alterations are not easily detectable by pianists, and do not pose a large impact on the ability to play. Limitations in this study include the small sample size and small number of key alterations. Preliminary analysis identifying low pianist perception to mass modifications allows future research with altered piano keys to take place without impacting performance. 


\subsection{Impact}

In regards to the development of a modified piano key, these results provide significant insights. Preliminary analysis identified low pianist perception to physical modifications. This allows future research with altered piano keys to take place without concern for perceivable impact to keyboard touch and response. Confident responses were low to not present across all of the mass alterations applied in the testing. The original concern that small modifications would negatively impact playing, was resolved from assessment of the study findings. Not only were the majority of participants unable to identify altered keys, but they incorrectly selected unmodified keys. These preliminary findings suggest a low sensitivity to alterations, as well as a lower focus towards the keytouch feel than previously expected. Participants were unable to make confident conclusions during scale testing, and struggled to correctly identify keys during investigative testing. Furthermore, the weight distribution may be of more importance than the weight itself, with focus on the centre of mass and mass moment of inertia. The initial findings of the study may reflect that if a weight increase is required, a shifted weight distribution to the front of the key may be preferable for this design. 


\section{Chapter 5}

\section{Theoretical Design Analysis}

As Chapter 3 outlined, the final design selected was to develop a key-specific sensor with the use of strain gauges integrated as a sensing device in a piano key. In general, the replica key would have material removed to provide space to integrate the sensor. We selected a simple approach to the sensor geometry using a manufactured small beam. This is affixed to the front and back portions of the key, with a white keytop allowing the beam to remain visually undetectable by the user. Mounting strain gauges to the beam allows measurements to be completed, as loading would go through the beam, and allow the desired input variables to be collected. To finalize the beam geometry, beam material, gauge selection, and gauge placement, further analysis was required.

The following Chapter outlines the approach used to develop a design for the custom key sensor. Section 5.1 provides a description of the general system which was used to establish a reference for the design. Section 5.2 outlines a theoretical mathematical analysis of the stresses and strains experienced across the parametrized design. To validate the unique values that would be recorded, mathematical simulations were completed, which is outlined in Section 5.3. 


\subsection{Definition of the System}

\subsubsection{Coordinate System}

A coordinate system was defined to establish a reference for the data collected from the sensing system. The right-handed coordinate system depicted in Figure 5.1 below was used throughout the analysis. The coordinate system was fixed to the key, and therefore allowed any coordinate to be relative to the key regardless of the key position. This system was further defined directionally from the perspective of a pianist. The z-direction can be described as the direction perpendicular to the keytop. The positive z-direction is defined as a perpendicular movement from the keytop away from the keyboard, commonly referenced as the normal force in previous studies. The x-direction can be described as the direction moving across the keys of the keyboard from bass to treble. The positive $\mathrm{x}$-direction is therefore the movement towards the right, or treble end, from the perspective of a pianist. The y-direction can be described as the movement parallel to the keytop. The positive y-direction is therefore the movement towards the body of the piano.

Furthermore, the origin of the coordinate system was established. The origin was set with consideration of the location of a finger touch. To allow a positive value in the $y$-direction, the origin of the $y$-axis was set as the front edge of the key. The location of the finger touch in the $\mathrm{x}$-direction may occur along the central body of the key or at a key edge. Placement of the origin of the $\mathrm{x}$-axis along the centre of the key front allows for simplified understanding of the symmetry of touch. Finally, the origin of the z-axis was defined. Similar to the x-axis placement, the z-axis origin was oriented at the centre of the front key height. This origin and coordinate system is displayed in Figure 5.1. 


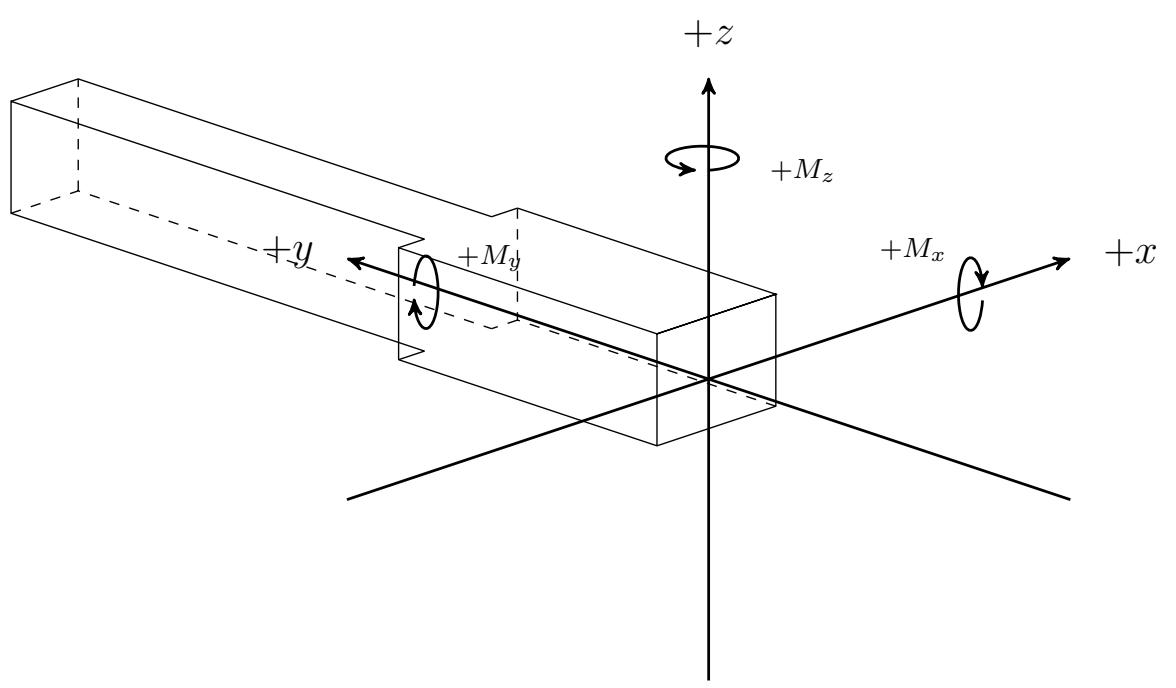

Figure 5.1: Key Orientation with respect to coordinate system

\subsubsection{Finger Contact Position}

The location of a key touch, or finger contact, is defined with respect to the coordinate system of the key. The position of a finger contact can vary across the top key surface within the portion of the key visible to the pianist. This defined area of a piano key is simplified by the top surface of a piano key which is covered with a keytop. In a realistic playing scenario, a white key would be played with a fixed z-position, corresponding to the keytop. For uniformity in the analysis, it was assumed that the z-position remains an unknown variable. The coordinates of the contact position was denoted as $x_{c}, y_{c}$, and $z_{c}$. These variables remain unique to each keystroke. In this analysis, the measurement of finger contact was a measure of the centre of pressure from a key touch.

The position of finger contact also is the location of the resultant force applied from the keystroke. The applied force, noted $\vec{F}$, was de-constructed as a force vector composed of three directional force components. The components are defined as $\left(F_{x}, F_{y}, F_{z}\right)$. The magnitude and direction of the applied force vector $\vec{F}$ may be 


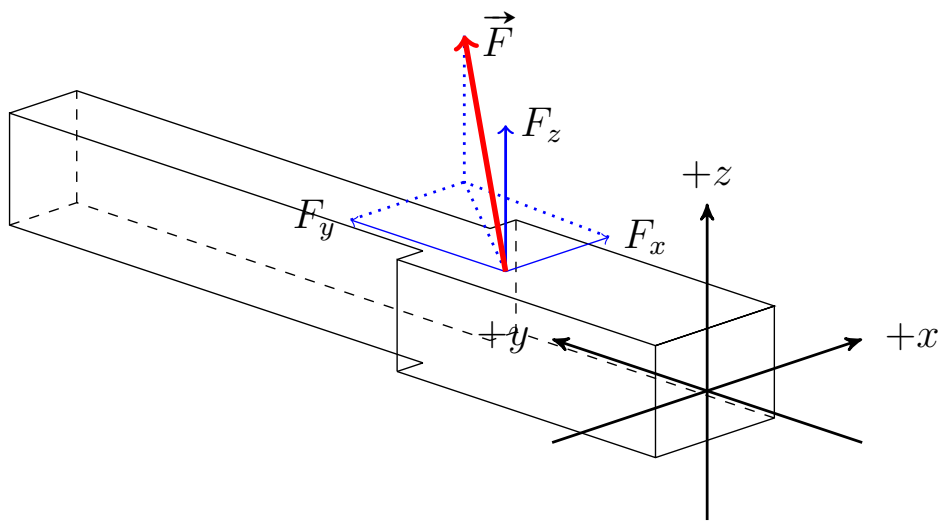

Figure 5.2: Finger-force Application

unique with each keystroke and participant. An example force application is shown in Figure 5.2.

\subsubsection{Strain Gauge Placement Position}

As outlined in Section 3.3, a key-specific sensor would be achievable through the implementation of strain gauges in a parametrized or over-parametrized design. To provide effective measurement, it was determined that the specific position of each sensor would be defined to optimize the ability for a gauge to detect measurement from one loading direction. However, redundancies in the measurements could be possible through the addition of extra gauges which would measure the same parameters. The level of redundancy necessary was evaluated through this analysis. The selection of this design evaluation process required specific definition of each strain gauge position in the key-specific sensor. The coordinate positions of each measurement location were constant. The coordinates of each gauge location of interest were denoted by $x_{i}, y_{i}$, and $z_{i}$, where $i$ denotes the strain gauge of interest. The arrangement of the strain gauges was fixed within the sensing beam which was part of the piano key, as shown in Figure 5.3. 


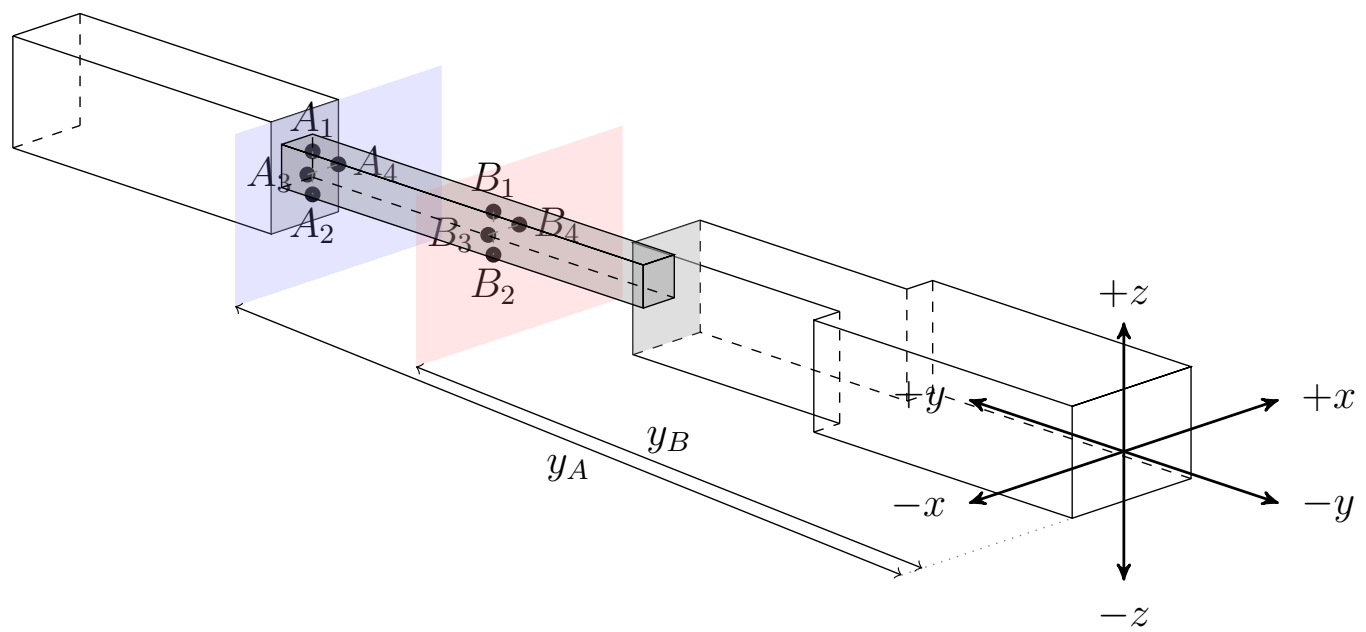

Figure 5.3: Position of Planes A and B with respect to the Origin

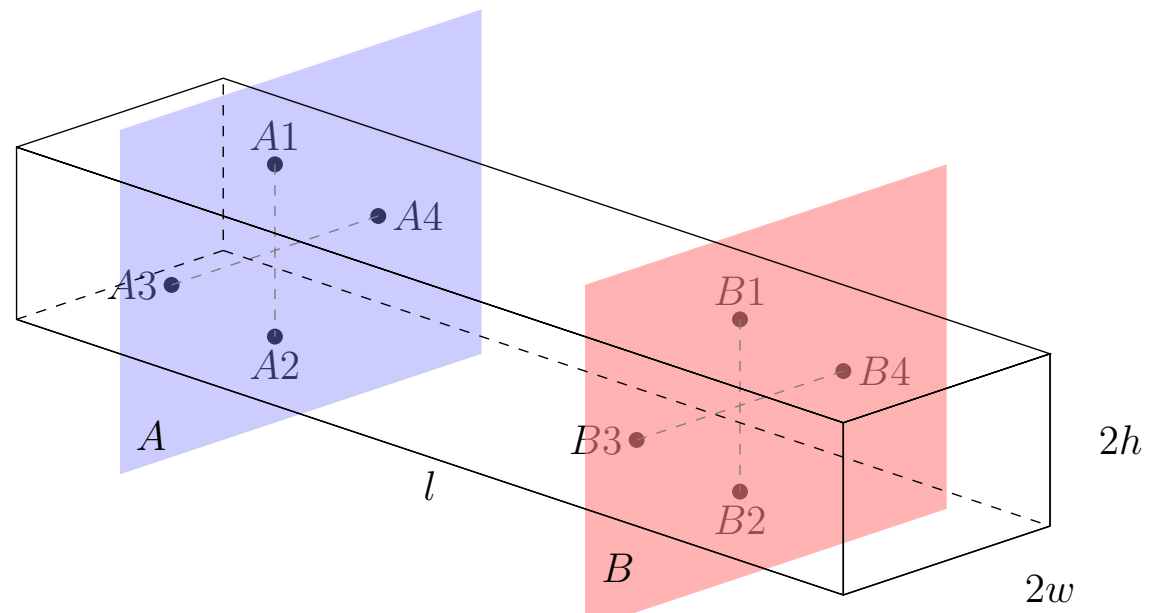

Figure 5.4: Strain Gauge Rosette Positions Across Beam 
To ensure all input metrics of interest were measured, two positions for the strain gauge placement were selected; defined as Plane $A$ and Plane $B$. Integration of two testing planes allowed redundancy in the theoretical analysis of strain measurements. The symmetry in the positions of interest between Plane $A$ and Plane $B$ would result in similar reactions to loading directions. Additionally, two planes would allow distinction of position. As will be further outlined further in Section 5.2.4, the directional position between the point of loading application and the measurement gauge with the resultant strain reading are related. The two planes would therefore allow comparison between the data readings, which would introduce the ability to complete position measurement. Each strain gauge was therefore defined by the plane it lies on, as well as its position within that plane. Within the planes, it was concluded that an effective method to limit the inputs affecting a single gauge would be to place gauges along a neutral axis. The symmetry of the gauges on either side of the beam would also provide further comparison between loading directions as well as $\mathrm{x}$-position differentiation. For this analysis, four unique arrangements were selected for each plane. Therefore, when referring to a strain gauge of interest or a position $i$, it was defined as $A 1-A 4$ and $B 1-B 4$.

The size of the sensing beam in which the strain gauges are placed was defined by general dimensions to provide meaningful coordinates for the strain gauge positions. These dimensions, as shown in Figure 5.4, were defined in terms of the variables $2 w$, $2 h$, and $l$. The geometry of the beam are further described and rationalized in Section 6.1.2. The specific coordinates of a gauge were represented by the coordinates with respect to the beam geometry. As the design involves two measurement planes, the y-positions of the gauges were defined by the location of Plane $A$ and Plane $B$ with respect to the datum. These variables of distance are defined as $y_{A}$ and $y_{B}$. These constant coordinates, which are outlined below, are therefore used when describing a 
strain gauge position.

$$
\begin{gathered}
A 1\left(0, y_{A}, h\right) \\
A 2\left(0, y_{A},-h\right) \\
A 3\left(-w, y_{A}, 0\right) \\
A 4\left(w, y_{A}, 0\right) \\
B 1\left(0, y_{B}, h\right) \\
B 2\left(0, y_{B},-h\right) \\
B 3\left(-w, y_{B}, 0\right) \\
B 4\left(w, y_{B}, 0\right)
\end{gathered}
$$

\subsection{Theoretical Stress and Strain Analysis}

\subsubsection{Purpose}

The decision to produce a key-specific sensor beam through the use of strain gauges required verification that the final design would meet the specifications outlined in Section 3.2. The unique orientation of the strain gauges, the geometry of the sensing beam, and the material selected would all significantly influence the ability to collect sufficient unique measurements. As 6 unknown variables exist, $F_{x}, F_{y}, F_{z}, x_{c}, y_{c}$, and $z_{c}$, it was critical to confirm these measurements were possible. It was determined that a method to complete this verification was to develop a theoretical stress analysis.

As outlined in Figure 5.5, completing a theoretical stress analysis could then be used to reach the desired output; that of the expected strain outputs of a strain gauge placed along Plane $A$ and Plane $B$. To achieve the strain outputs, the stresses would be assessed, and then equated to the strain outputs through stress and strain 


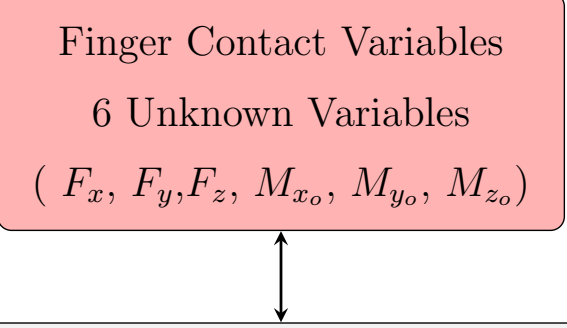

Directional Stresses Acting on Plane A and B 12 Stress Expressions Developed

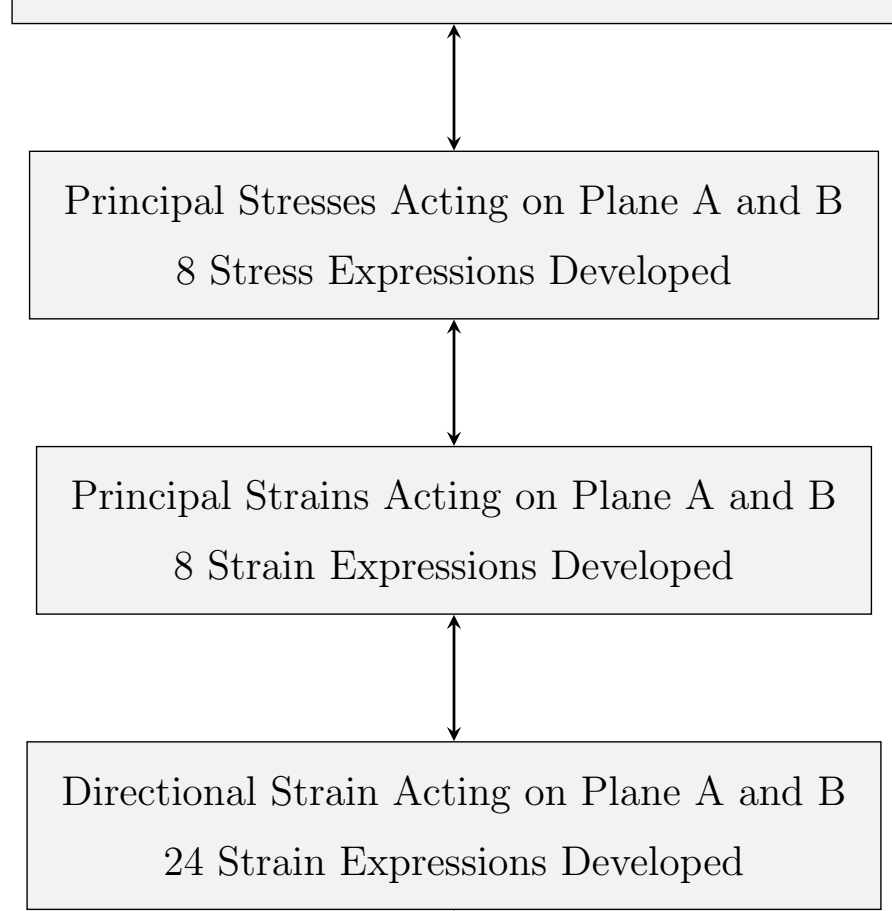

Measured Strain Outputs from Strain Gauges Positioned along Plane A and B 12 Half-Bridge Strain Outputs

Figure 5.5: Stress to Strain Conversion Flowchart 
theory. As the calculated stress is dependent on the measurement position of interest, expressions were required across both the directional planes of interest, being that of the $x-y$ and $z-y$ planes, as well as the positional planes, Plane $A$ and Plane $B$. These findings were further used to complete the outlined approach to calculate the theoretical strain. Through this method, it was confirmed if sufficient strain values were produced to be measured by strain gauges, and if the design would meet the previously described measurement specifications. The following section outlines the algebraic analysis completed using static body stress analysis. Section 5.2.3 provides an overview of these general theoretical equations.

\subsubsection{Assumptions}

Simplifying assumptions are necessary to complete the theoretical stress analysis and apply loading theories [61, for reference to loading theory]. It should be made clear that these assumptions were put in place for the analysis of the system. One initial assumption is that of a static system. This would allow the stress in the system to be analyzed at specific force and position applications. Furthermore, a static analysis would allow an assessment of the design's ability to safely withstand the applied loading conditions. It should be noted that the system would still work in a dynamic environment. However, we would additionally need to know the total acceleration in order to find the contact force. Therefore, to simplify the analysis of the data, this assumption has been put in place for the analysis.

Additionally, it was assumed that both the sensing beam and the replica key were of homogenous materials. This assumption was used to justify the material strength properties utilized in the analysis. Additionally, it was assumed that the geometry of the sensing beam was straight and free of stress when external loads were removed. 
During the analysis of the stress at defined positions on the sensing beam, a simplifying assumption of gauge placement was considered. The positions of interest were considered to be sufficiently far from the point of load application and stress concentrations, which may be caused by surface holes or connections of the beam. This assumption was necessary for the analysis of the system, as a close position to the load application causes greater local stress effects, which would impact the accuracy of assessing the true stresses across the beam. In the functional assembly and testing of the manufactured sensing beam, these factors would, if necessary, be taken into consideration for data collection and data validation.

Finally, this analysis was made with respect to the entire key system. Therefore, the effect of the key front applied to the end of the sensing beam had to be assessed. When fully assembled, the key will apply a weight on the system. However, in this

analysis we are considering the full assembly without external loading as the zero strain case. The assumption of the static equilibrium, zero strain position, occurring with the key front assembly allows measurement of the deflection, and strain, from this static equilibrium point. This analysis assumes all measurements are made with respect to the static equilibrium position.

\subsubsection{Applied Stress States}

As Figure 5.5 outlines, the first step in analysis was to calculate the applied stresses on the beam. To do so, the general expressions for the applied stress states were defined. Each unique keystroke may result in different loading conditions on a piano key. As the intent of the analysis was to consider the effect of the 6 unknown contact variables, it is critical to consider all components that may influence the total stress acting on a point in the sensing beam. This was required to ensure sufficient measurements were taken to measure the components of force and their position which 
influence the stress applied on the key. The following section provides a general overview of the expected stresses applied to the beam.

\section{Axial Stress}

A finger-force application can result in axial stress. Axial stress which, in this application, is developed on a sensing beam face perpendicular to the applied load, provides a theoretical average stress across the cross-section of interest. The average stress is determined by dividing the applied load by the cross-sectional area. The direction of the applied load will determine if the result of the loading is tensile stress or compressive stress. The standard formula for axial stress is given below as,

$$
\sigma_{\text {axial }}=\frac{F_{\text {axial }}}{A_{\text {cross-section }}}
$$

as shown by [61, Eq. 4.1].

\section{Bending Stress}

A finger-force application will generally result in bending stress, which occurs when a normal stress is acted on a point which causes the element to bend. The bending stress is therefore calculated as a function of the moment created by the applied load, the distance the point of interest is located from the neutral axis of the beam, and the second moment of area of the sensing beam. As the coordinate planes were set on the neutral axis, the distance from the neutral axis is the respective coordinate distance of each strain gauge. The distance variable applied in the equation is dependent on the force which creates an applied moment. Therefore variable $d_{i}$ was introduced to represent the perpendicular distance from the neutral axis of interest. The general equation for the bending stress, 


$$
\sigma_{\text {bending }}=\frac{M \cdot d}{I}
$$

as shown by [61, Eq. 4.6].

The second moment of area of the beam can be expressed in terms of the previously defined variables of $2 w$ and $2 h$. This develops Equations 5.3 and 5.4, as shown through the general equations in [61, pg. 813].

$$
\begin{aligned}
& I_{x x}=\frac{1}{12} \cdot(2 h)(2 w)^{3} \\
& I_{z z}=\frac{1}{12} \cdot(2 w)(2 h)^{3}
\end{aligned}
$$

\section{Shear Stress}

A finger-force application may create a shear stress on the sensing beam. A shear stress may be developed when the a load is applied parallel to a material cross-section. Shear loading can be further defined as direct or torsional. The direct component of shear stress is experienced with negligible bending. Therefore, the maximum stress is determined through dividing the applied load by the area of the surface element the load is being applied across and a applying a geometric ratio [61]. The torsional component of shear stress is developed when off-axis loading results in a rotational force on the element. The maximum stress developed is therefore dependent on the moment caused by the applied load and is a function of the geometry of the sensing beam. The position of maximum shear stress for a rectangular cross-section would be along the neutral axis [61].

$$
\tau_{\text {max, direct }}=\frac{3}{2} \cdot \frac{F_{\text {shear }}}{A_{\text {parallel }}}
$$


as proven by [61, Eq. 4.14].

$$
\tau_{\text {max,torsion }}=\frac{(3 a+1.8 b)}{a^{2} b^{2}} \cdot M
$$

as proven by [61, Eq. 4.5], where the variables $a$ and $b$ represent the cross-sectional width and height of the beam with respect to the plane of interest, respectively.

\subsubsection{Development of Moment Equations}

A method to determine the position of a finger touch during a keystroke, is to interpret the moments developed from the finger touch with respect to a strain gauge position. A general moment expression about a strain gauge location, will involve two position variables to develop a moment arm. This is due to the moment being dependent on the position of the finger relative to the strain gauge location. Therefore, the position variables of interest are the constant strain gauge position, which in general can be referred to a position $i$ on the beam, and the unique variable of the finger contact position, previously defined by the coordinates $\left(x_{i}, y_{i}, z_{i}\right)$. The finger contact position is an unknown variable, see equations 5.7, 5.8, and 5.9. Through this relationship, a larger moment arm would result in a larger total value of the moment. As the moment is used in the previous equations to develop stress, and the resulting strain, relationships, the size of the moment arm therefore impacts the final strain output.

$$
\begin{aligned}
& M_{x}=F_{z} \cdot\left(y_{i}-y_{c}\right)-F_{y} \cdot\left(z_{i}-z_{c}\right) \\
& M_{y}=F_{x} \cdot\left(z_{i}-z_{c}\right)-F_{z} \cdot\left(x_{i}-x_{c}\right)
\end{aligned}
$$




$$
M_{z}=F_{y} \cdot\left(x_{i}-x_{c}\right)-F_{x} \cdot\left(y_{i}-y_{c}\right)
$$

The equations provide a suitable moment expression; however, it is of interest to isolate the unknown position variables further. This can be achieved by rearranging these equations through the expression of the moment about the origin. An equivalent force system can be realized at the origin. These moment arm expressions would therefore only include the unknown variables of the contact position, as shown in Equations 5.10, 5.11, and 5.12.

$$
\begin{aligned}
& M_{x_{o}}=F_{z} \cdot y_{c}-F_{y} \cdot z_{c} \\
& M_{y_{o}}=F_{x} \cdot z_{c}-F_{z} \cdot x_{c} \\
& M_{z_{o}}=F_{y} \cdot x_{c}-F_{x} \cdot y_{c}
\end{aligned}
$$

If Equations 5.10, 5.11, and 5.12 are substituted into Equations 5.7, 5.8, and 5.9, respectively, the expressions are greatly simplified. Substituting the above equations develops the final expressions, below. The final expressions are significant in the analysis as $M_{x_{o}}, M_{y_{o}}$, and $M_{z_{o}}$ isolate the unknown contact variables which are required to be solved. Throughout the following stress analysis the moments about the origin of each axis can be expressed as an unknown which is to be solved for.

$$
\begin{aligned}
& M_{x}=F_{z} \cdot y_{i}-F_{y} \cdot z_{i}-M_{x_{o}} \\
& M_{y}=F_{x} \cdot z_{i}-F_{z} \cdot x_{i}-M_{z_{o}} \\
& M_{z}=F_{y} \cdot x_{i}-F_{x} \cdot y_{i}-M_{y_{o}}
\end{aligned}
$$




\subsubsection{Development of Stress Expressions}

The first step in the analysis was to apply the defined applied stress states to the system, being that of the sensing beam. The stresses acting on the sensing beam are dependent on the orientation of the forces applied and the measurement point of interest. Although all measurement points of interest lie in either the defined Plane A or Plane $B$ their coordinate positions fall within the $x-y$ and $z-y$ planes. Figure 5.6 displays an example of the possible applied forces during a keystroke on the sensing beam.

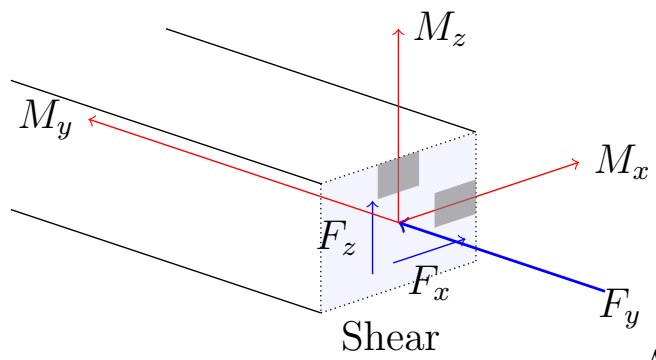

Axial

Figure 5.6: Loads on Measurement Cross-Section (x-z plane)

Completion of a theoretical stress analysis began with the visualization of an element on the sensing beam, as in Figure 5.7. The stress on the top surface and left side of the beam provided the necessary general equations to evaluate the positions of interest in which strain gauges would be placed. Therefore, visualization of the extraction of an element from either the top or side surface would allow the necessary expressions to be derived for the full stress analysis. The expressions would be further applied to the four corresponding positions outline previously for both Plane A and Plane $B$. This would be required to eventually compare the strains expected at the required measurement positions. 


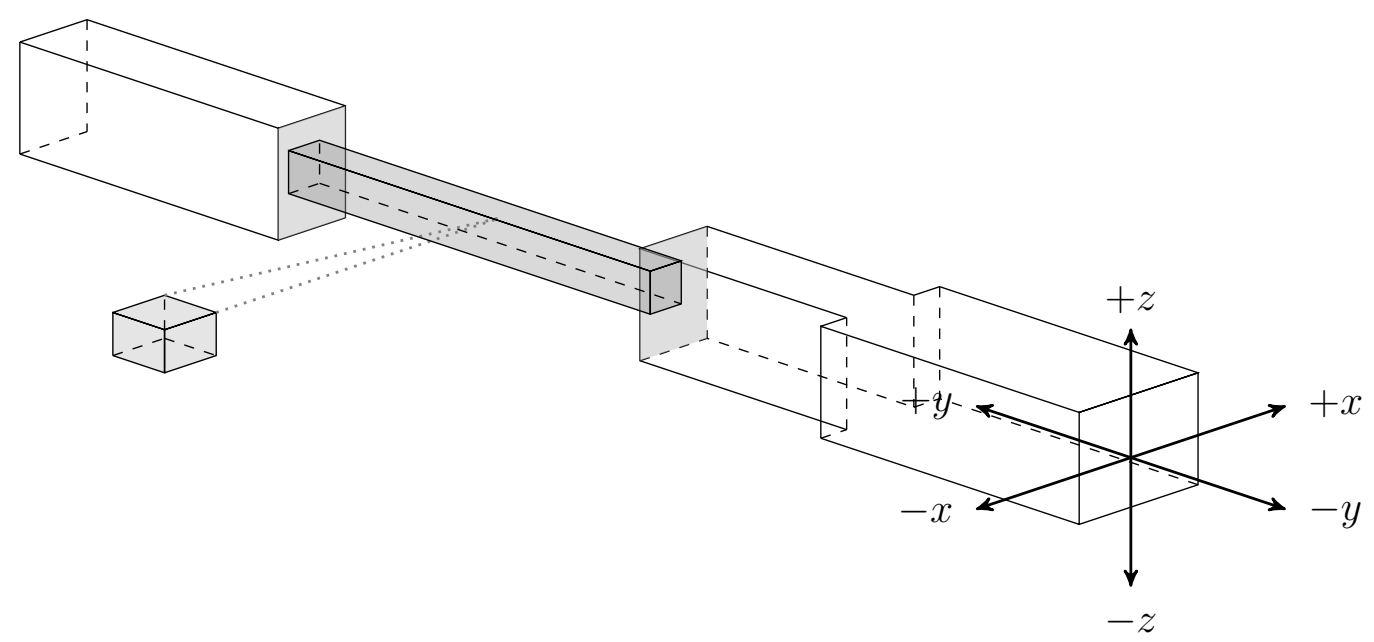

Figure 5.7: Extraction of an Element at the Top of the Sensing Beam

The applied stress states on an element can be expressed in general. For any element of the sensing beam, the following conditions are held true. All elements are surface elements on the neutral axis, therefore:

1. The free surface of the element has no stress, shear or normal

2. The lateral surface has stresses due to shear (due to torsion and direct shear forces)

3. The axial surface has stresses due to bending, axial load, and shear (due to torsion and direct shear forces)

These conditions on the free, lateral, and axial surfaces can be further related to the directional stresses for an element on the top/bottom or side surface of the sensing beam. The expressions in the following sections were developed based on the general formulas outlined in Section 5.2.3. 


\section{Top/Bottom Surface Element Stress Components}

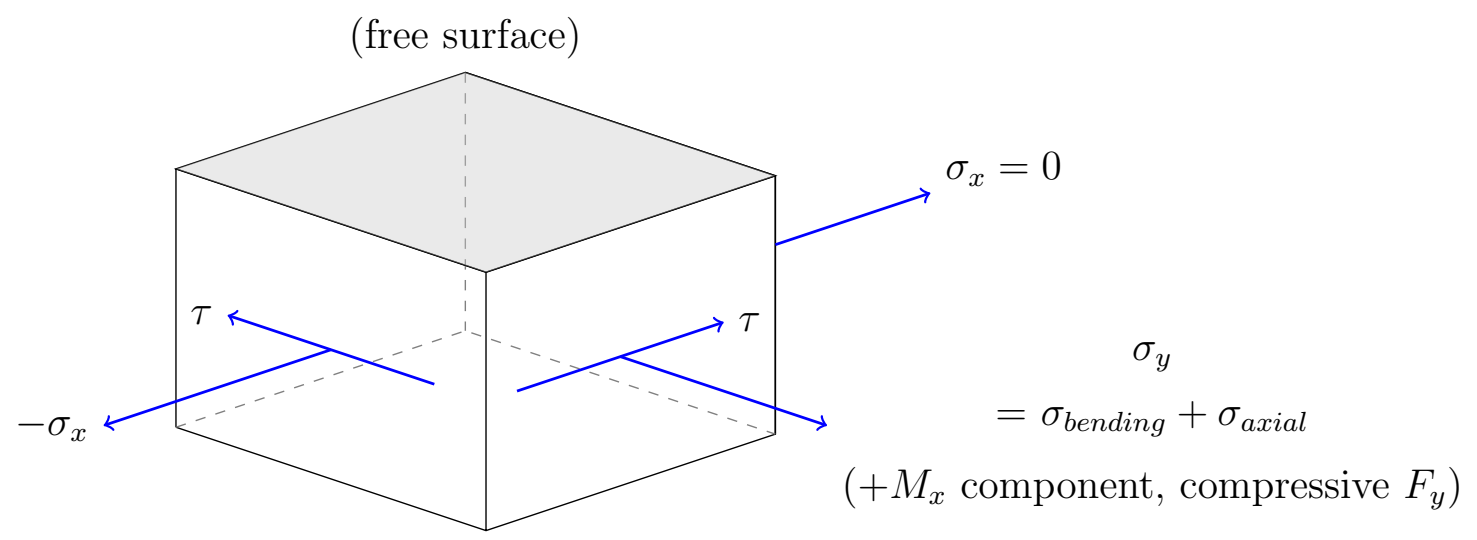

Figure 5.8: Stress Acting on the x-y Plane

An element from Figure 5.7 was first studied in consideration of the top and bottom surface of the sensing beam. Extraction of the element provided visualization of the stresses which act at this point. This was depicted in Figure 5.8. In the case of an element on the top or bottom of the sensing beam, a variation in the equation variables would be noted when comparing the distance from the neutral axis. The respective distance from the neutral axis for this stress element was that of the zposition from the neutral axis, which looking at the top or bottom surface of the sensing element would be \pm . Equations developed between Plane $A$ and Plane $B$ would be identical, with the exception of the $y$-distance variable $y_{A}$ and $y_{B}$ which is used in calculation of the $M_{z}$ moment. In general, for any element on the top or bottom surface, the following conditions are true:

1. The normal stress on the axial surface is due to the direct load $F_{y}$ and the bending created by $M_{x}$ (see Equation 5.16).

2. The normal stress on the lateral surface is a bending stress, due to $M_{z}$. As the element is on the neutral axis the bending stress is zero (see Equation 5.17). 
3. The shear stress on the lateral and axial surfaces are equal in magnitude. The direct shear is due to $F_{x}$ and the torsional shear is due to $M_{y}$ (see Equation 5.18, [61, Eq. 4.4 and 4.5]).

The general expressions of the stresses were then developed with these conditions in place.

\section{General Expressions}

As depicted in Figure 5.8, the normal stress in the y-direction would be developed due to axial and bending stress. If the force component acted along the positive y-axis it would act towards the sensing beam, which would develop a compressive force. This would result in a compressive axial stress, which produces a negative stress component. Therefore, in the general expression a negative value is presented with a positive $F_{y}$ axial force. The only bending moment was developed from $M_{x}$.

$$
\begin{gathered}
\sigma_{y}=\sigma_{\text {bending }_{y}}+\sigma_{\text {axial }_{y}} \\
\sigma_{y}=\frac{M_{x}}{I_{x x}} \cdot z_{i}+\frac{M_{z}}{I_{z z}} \cdot x_{i}-\frac{F_{\text {axial }}}{A_{\text {cross-section }}} \\
\sigma_{y}=\frac{M_{x}}{I_{x x}} \cdot z_{i}+\frac{M_{z}}{I_{z z}} \cdot x_{i} \frac{0}{2 w \cdot 2 h} \\
\sigma_{y}=\frac{F_{y}}{I_{x x}} \cdot z_{i}-\frac{F_{y}}{2 w \cdot 2 h}
\end{gathered}
$$

As outlined in Figure 5.8, along the neutral axis no stress is developed in the $\mathrm{x}$ direction.

$$
\begin{gathered}
\sigma_{x}=\sigma_{\text {bending }_{x}} \\
\sigma_{x}=\frac{M_{z}}{I_{z z}} \cdot x_{i}
\end{gathered}
$$




$$
\sigma_{x}=\frac{M_{z}}{I_{z z}} \cdot \not \overbrace{i}^{0}
$$

$$
\sigma_{x}=0
$$

A shear stress would be developed due to shear forces and torsion. The direct shear force would be present due to the $F_{x}$ component of the force applied, across the crosssectional area of the sensing beam, [61, see Eq. 4.4 and 4.5]. These equations could be expressed with respect to the plane of interest.

$$
\begin{gathered}
\tau_{x y}=\tau_{\text {max } \text { direct }}+\tau_{\text {max } \text { torsion }} \\
\tau_{x y}=\frac{3}{2} \cdot \frac{F_{\text {shear }}}{A_{\text {cross-section }}}+\frac{(3 \cdot 2 w+1.8 \cdot 2 h)}{(2 w)^{2} \cdot(2 h)^{2}} \cdot M_{y} \\
\tau_{x y}=\frac{3}{2} \cdot \frac{F_{x}}{2 w \cdot l}+\frac{(3 \cdot 2 w+1.8 \cdot 2 h)}{(2 w)^{2} \cdot(2 h)^{2}} \cdot M_{y}
\end{gathered}
$$

\section{Application of Equations to the Strain Gauge Positions of Interest}

The relationships defined from Figure 5.8 can be further applied to the predetermined positions of interest for strain gauge placement. These relationships can be developed along both Plane $A$ and Plane $B$, where the variance between the expressions would be the $\mathrm{y}$-axis distance position of $y_{A}$ and $y_{B}$. Therefore, the following equations were only represented for Plane $A$. The general equations were applied to the strain gauge positions of interest being position $A 1$ and $A 2$. First, the general positions in Equations 5.16, 5.17, and 5.18 were replaced with the appropriate position variables at $A 1$. 
At position $A 1$, the value of $+h$ was substituted into Equation 5.16 for the variable $z_{i}$. When the bending moment about the x-axis was expanded, the value of $y_{A}$ was also substituted for the variable $y_{i}$. This expression can be rearranged to isolate the unknown variables, being $F_{z}, F_{y}$, and $M_{x_{o}}$. It should be noted that the process of rearranging and isolating the unknown variables produced a negative coefficient for the moment about the $\mathrm{x}$-axis at the origin. This expression was due to the expansion of the moment about the $\mathrm{x}$-axis to include the moment at the position of interest and the moment at the origin of the system. This rearrangement resulted in a component of the moment expression to contribute a negative value to the stress expression.

$$
\sigma_{y, A 1}=\frac{M_{x}}{I_{x x}} \cdot z_{A 1}-\frac{F_{y}}{2 w \cdot 2 h}
$$

expanding,

$$
\sigma_{y, A 1}=\frac{h}{I_{x x}} \cdot\left[F_{z} \cdot y_{A}-F_{y} \cdot h-M_{x_{o}}\right]-\frac{F_{y}}{2 w \cdot 2 h}
$$

rearranging,

$$
\sigma_{y, A 1}=\frac{h \cdot y_{A}}{I_{x x}} \cdot F_{z}+\left(-\frac{h^{2}}{I_{x x}}-\frac{1}{2 w \cdot 2 h}\right) \cdot F_{y}-\frac{h}{I_{x x}} \cdot M_{x_{o}}
$$

As previously defined, there is no $\sigma_{x}$ component of stress present at this point of interest.

$$
\sigma_{x, A 1}=0
$$

Equation 5.18 can be expressed at position $A_{1}$. This was achieved through expanding the moment expression of $M_{y_{o}}$. This expansion uses the value of $z_{i}$ as $+h$. After cancellation due to the position along the neutral axis, the reduced moment expression from the torsional component of shear stress can then be rearranged with the direct component of shear stress to isolate the unknown variables in this expression, 
being $F_{z}$, and $M_{y_{o}}$.

$$
\tau_{x y, A 1}=\frac{3}{2} \cdot \frac{F_{x}}{2 w \cdot l}+\frac{(3 \cdot 2 w+1.8 \cdot 2 h)}{(2 w)^{2} \cdot(2 h)^{2}} \cdot M_{y}
$$

expanding,

$$
\tau_{x y, A 1}=\frac{3}{2} \cdot \frac{F_{x}}{2 w \cdot l}+\frac{(3 \cdot 2 w+1.8 \cdot 2 h)}{(2 w)^{2} \cdot(2 h)^{2}} \cdot\left[F_{x} \cdot h-F_{z} \cdot x_{A \top}-0\right.
$$

rearranging,

$$
\tau_{x y, A 1}=\left[\frac{3}{2 \cdot(2 w \cdot l)}+\frac{(3 \cdot 2 w+1.8 \cdot 2 h)}{(2 w)^{2} \cdot(2 h)^{2}} \cdot h\right] \cdot F_{x}-\frac{(3 \cdot 2 w+1.8 \cdot 2 h)}{(2 w)^{2} \cdot(2 h)^{2}} \cdot M_{y_{o}}
$$

Analysis at position $A 2$ would produce an identical procedure, with the change of $-h$ being substituted for $z_{i}$. This analysis and isolation of unknown variables would develop the final expressions below.

$$
\begin{gathered}
\sigma_{y, A 2}=\frac{-h \cdot y_{A}}{I_{x x}} \cdot F_{z}+\left(-\frac{h^{2}}{I_{x x}}-\frac{1}{2 w \cdot 2 h}\right) \cdot F_{y}+\frac{h}{I_{x x}} \cdot M_{x_{o}} \\
\sigma_{x, A 2}=0 \\
\tau_{x y, A 2}=\left[\frac{3}{2 \cdot 2 w \cdot l}+\frac{(3 \cdot 2 w+1.8 \cdot 2 h)}{2 w^{2} \cdot 2 h^{2}} \cdot(-h)\right] \cdot F_{x}-\frac{(3 \cdot 2 w+1.8 \cdot 2 h)}{2 w^{2} \cdot 2 h^{2}} \cdot M_{y_{o}}
\end{gathered}
$$

\section{Left/Right Surface Element Stress Components}

An element from Figure 5.7 was then studied at the left and right surfaces of the sensing beam, as depicted in Figure 5.9. A visualization of the stresses acting on 


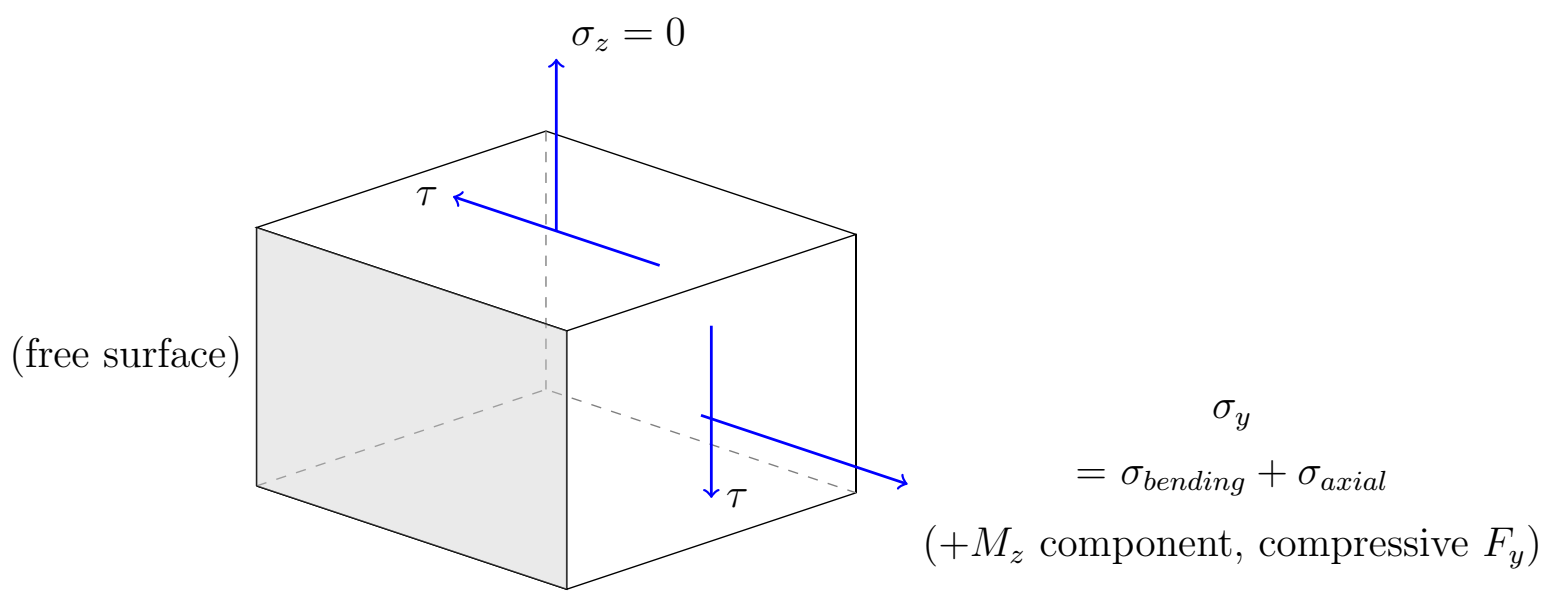

Figure 5.9: Stress Acting on the z-y Plane

an element along the left or right surface is shown in Figure 5.9. In the case of an element on the left or right side of the sensing beam, a variation in the equation variables would be noted when comparing the distance from the neutral axis. The respective distance from the neutral axis for this stress element was that of the $\mathrm{x}$ position from the neutral axis, which looking at the top or bottom surface of the sensing element would be $\pm w$. Equations developed between Plane $A$ and Plane B would be identical, with the exception of the y-distance variable $y_{A}$ and $y_{B}$ which is used in calculation of the $M_{z}$ moment. In general, for any element on the side surface, the following conditions are true:

1. The normal stress on the axial surface is due to the direct load $F_{y}$ and the bending created by $M_{z}$ (see Equation 5.25).

2. The normal stress on the lateral surface is a bending stress, due to $M_{x}$. As the element is on the neutral axis the bending stress is zero (see Equation 5.26).

3. The shear stress on the lateral and axial surfaces are equal in magnitude. The direct shear is due to $F_{z}$ and the torsional shear is due to $M_{y}$ (see Equation 5.27, [61, Eq. 4.4 and 4.5]). 
The general expressions of the stresses were then developed.

\section{General Expressions}

As depicted in Figure 5.9, a normal stress in the y-direction would be developed due to axial and bending stress. The axial force applied in this orientation would be the same expression developed in Figure 5.8. Recall, a negative value is presented with a positive $F_{y}$ axial force due to the compressive stress which would occur when a positive $F_{y}$ force is applied. A bending moment would be developed from $M_{z}$.

$$
\begin{gathered}
\sigma_{y}=\sigma_{\text {bending }_{y}}+\sigma_{\text {axial }_{y}} \\
\sigma_{y}=\frac{M_{x}}{I_{x x}} \cdot z_{i}+\frac{M_{z}}{I_{z z}} \cdot x_{i}-\frac{F_{\text {axial }}}{A_{\text {cross }- \text { section }}} \\
\sigma_{y}=\frac{M_{x}}{I_{x x}} \cdot z_{i}+\frac{M_{z}}{I_{z z}} \cdot x_{i}-\frac{F_{y}}{2 w \cdot 2 h} \\
\sigma_{y}=\frac{M_{x}}{I_{x x}} \cdot \not z i+\frac{M_{z}}{I_{z z}} \cdot x_{i}-\frac{F_{y}}{2 w \cdot 2 h} \\
\sigma_{y}=\frac{M_{z}}{I_{z z}} \cdot x_{i}-\frac{F_{y}}{2 w \cdot 2 h}
\end{gathered}
$$

As depicted in Figure 5.9, along the neutral axis no stress is developed in the zdirection.

$$
\begin{aligned}
\sigma_{z} & =\sigma_{\text {bending }_{z}} \\
\sigma_{z} & =\frac{M_{x}}{I_{x x}} \cdot z_{i} \\
\sigma_{z} & =\frac{M_{x}}{I_{x x}} \cdot \not \not_{i}^{0}
\end{aligned}
$$




$$
\sigma_{z}=0
$$

A shear stress would be developed due to shear forces and torsion. The direct shear force would be present due to the $F_{y}$ component of the force applied, across the cross-sectional area of the sensing beam. A torsional moment developed about the z-axis would also result in a shear stress component being developed due to torsion across the rectangular cross-section, [61, see Eq. 4.4 and 4.5].

$$
\begin{gathered}
\tau_{x y}=\tau_{\text {max,direct }}+\tau_{\text {max }, \text { bending }} \\
\tau_{z y}=\frac{3}{2} \cdot \frac{F_{y}}{A_{\text {cross-section }}}+\frac{(3 \cdot l+1.8 \cdot 2 w)}{l^{2} \cdot 2 w^{2}} \cdot M_{z} \\
\tau_{z y}=\frac{3}{2} \cdot \frac{F_{y}}{l \cdot 2 h}+\frac{(3 \cdot l+1.8 \cdot 2 w)}{l^{2} \cdot 2 w^{2}} \cdot M_{z}
\end{gathered}
$$

\section{Application to the Strain Gauge Positions of Interest}

The relationships defined from Figure 5.9 can be further applied to the predetermined positions of interest for strain gauge placement. As completed in the previous section, the following equations were only represented for Plane A, and therefore $y_{A}$ was substituted for the variable $y$. The general equations were applied to the strain gauge positions of interest being position $A 3$ and $A 4$.

Equations 5.25, 5.26, and 5.27 were substituted with the appropriate position variables at $A 3$. At position $A 3$, the value of $-w$ was substituted into Equation 5.25 for the variable $x_{i}$ to develop an expression for the normal stress. When the bending moment about the z-axis was expanded the value of $y_{A}$ was also substituted for the 
variable $y_{i}$. This expression can be rearranged to isolate the unknown variables, being $F_{x}, F_{y}$, and $M_{z_{o}}$.

$$
\sigma_{y, A 3}=\frac{M_{z}}{I_{z z}} \cdot\left(x_{A 3}\right)-\frac{F_{y}}{2 w \cdot 2 h}
$$

expanding,

$$
\sigma_{y, A 3}=\frac{-w}{I_{z z}} \cdot\left[F_{y} \cdot(-w)-F_{y} \cdot y_{A}-M_{z_{o}}\right]-\frac{F_{y}}{2 w \cdot 2 h}
$$

rearranging,

$$
\sigma_{y, A 3}=\frac{w \cdot y_{A}}{I_{z z}} \cdot F_{x}+\left[\frac{w^{2}}{I_{z z}}-\frac{1}{2 w \cdot 2 h}\right] \cdot F_{y}+\frac{w}{I_{z z}} \cdot M_{z_{o}}
$$

As previously defined, there is no $\sigma_{z}$ component of stress at the measurement position of interest.

$$
\sigma_{z, A 3}=0
$$

Equation 5.27 can be expressed at Position A3. This was achieved through expanding the moment expression of $M_{z_{o}}$. This expansion substitutes the values of $-w$ for the variable $x_{i}$ and $y_{A}$ for the variable of $y_{i}$. The torsional component of shear stress can then be rearranged with the direct component of shear stress to isolate the unknown variables in this expression, being $F_{x}, F_{y}$, and $M_{z_{o}}$.

$$
\tau_{z y, A 3}=\frac{3}{2} \cdot \frac{F_{y}}{l \cdot 2 h}+\frac{(3 \cdot l+1.8 \cdot 2 w)}{l^{2} \cdot 2 w^{2}} \cdot M_{z}
$$

expanding,

$$
\tau_{z y, A 3}=\frac{3}{2} \cdot \frac{F_{y}}{l \cdot 2 h}+\frac{(3 \cdot l+1.8 \cdot 2 w)}{l^{2} \cdot 2 w^{2}} \cdot\left[F_{y} \cdot(-w)-F_{x} \cdot y_{A}-M_{z o}\right]
$$


rearranging,

$$
\begin{array}{r}
\tau_{z y, A 3}=\left(-y_{A} \cdot \frac{(3 \cdot l+1.8 \cdot 2 w)}{l^{2} \cdot 2 w^{2}}\right) \cdot F_{x}+\left[\frac{3}{2 \cdot l \cdot 2 h}-\left(w \cdot \frac{(3 \cdot l+1.8 \cdot 2 w)}{l^{2} \cdot 2 w^{2}}\right)\right] \cdot F_{y} \\
-\frac{(3 \cdot l+1.8 \cdot 2 w)}{l^{2} \cdot 2 w^{2}} \cdot M_{z_{o}}
\end{array}
$$

Analysis at position $A 4$ would produce an identical procedure, with the change of $+w$ being substituted for $x_{i}$. This analysis and isolation of unknown variables would develop the following final expressions.

$$
\begin{gathered}
\sigma_{y, A 4}=\frac{-w \cdot y_{A}}{I_{z z}} \cdot F_{x}+\left[\frac{w^{2}}{I_{z z}}-\frac{1}{2 w \cdot 2 h}\right] \cdot F_{y}-\frac{w}{I_{z z}} \cdot M_{z_{o}} \\
\sigma_{z, A 4}=0 \\
\tau_{z y, A 4}=\left(-y_{A} \cdot \frac{(3 \cdot l+1.8 \cdot 2 w)}{l^{2} \cdot 2 w^{2}}\right) \cdot F_{x}+\left(\frac{3}{2 \cdot l \cdot 2 h}+w \cdot \frac{(3 \cdot l+1.8 \cdot 2 w)}{l^{2} \cdot 2 w^{2}}\right) \cdot F_{y} \\
-\frac{(3 \cdot l+1.8 \cdot 2 w)}{l^{2} \cdot 2 w^{2}} \cdot M_{z_{o}}
\end{gathered}
$$

\subsubsection{Theoretical Stress Matrix}

The approach to theoretically estimate the strain gauge outputs is outlined in Figure 5.5. Section 5.2.5 allowed development of the directional stresses on Plane A and Plane $B$. To further apply these equations to reach the strain gauge outputs, the theoretical relation between stress and strain were applied at the specific measurement locations of interest. This theoretical analysis would provide verification on the effectiveness of the proposed design. The theoretical strain readings from the gauge positions would be calculated to ensure they were within a measurable range. 
The first step to evaluate the effectiveness of the proposed design was at the level of the stress equations. Stress expressions from Section 5.2.5 can be further organized through a matrix. Development of a stress matrix allowed for isolation of known variables and the six unknown variables. This was required to develop further confirmation that the sensing beam arrangement would provide sufficient measurements to determine the applied loads based on the strain measurements. To obtain sufficient measurements, the stress matrix should contain six linearly independent row vectors. Development of a matrix would allow comparisons to identify row similarities.

The values of the coefficient matrix, for Plane $A$ only, are expressed below. These values were expressed in terms of the geometry of the sensing beam. The previously developed stress expressions were also isolated from the developed stress equations which were a function of the six unknown variables unique to each keystroke. The matrix was first arranged in order of stress expressions across each position of interest. 


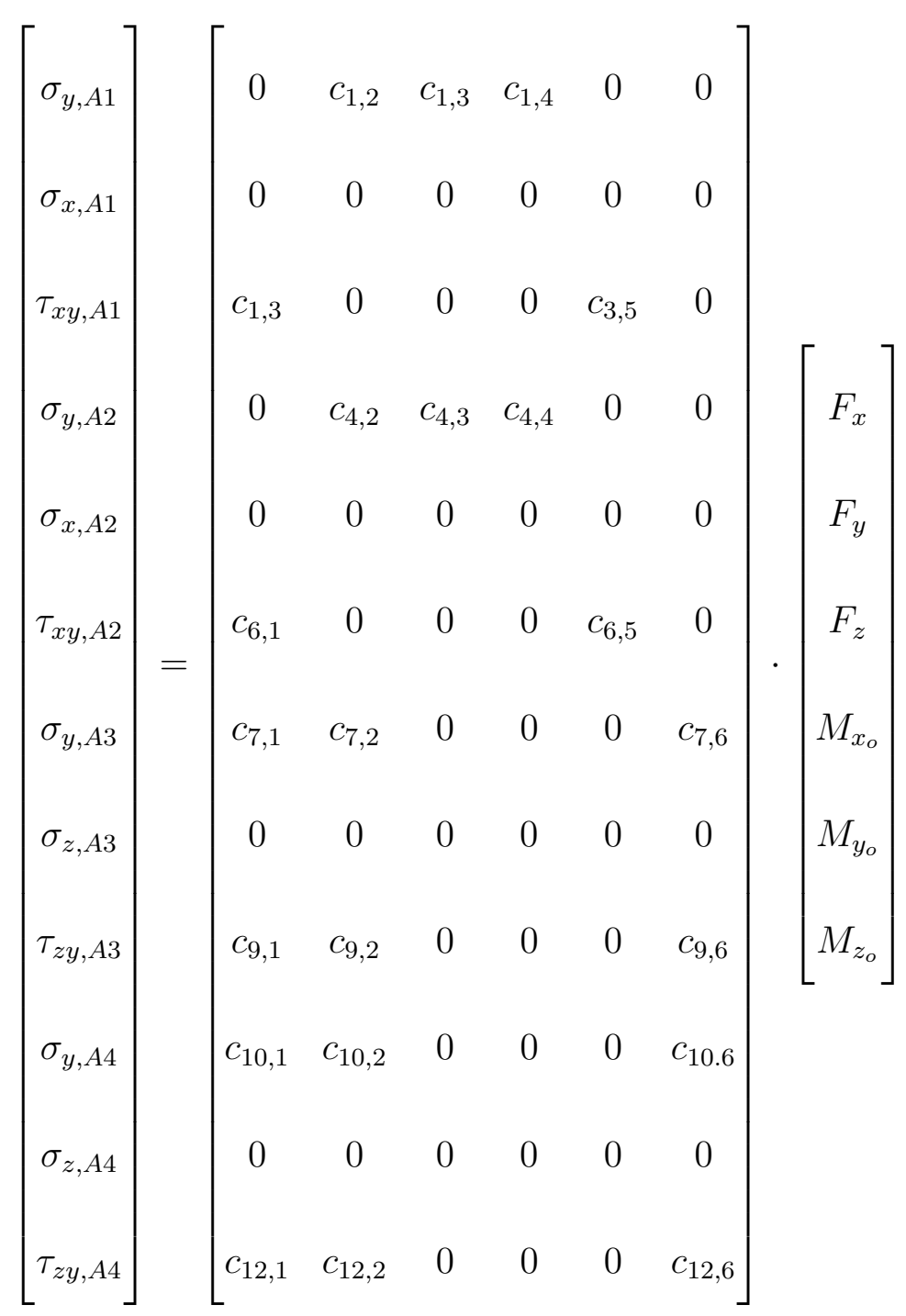

The values of the coefficient matrix are listed below. The original equations in which these terms were isolated from, can be reviewed in Section 5.2.5. 


$$
\begin{aligned}
& c_{1,2}=-\frac{h^{2}}{I_{x x}}-\frac{1}{2 w \cdot 2 h} \\
& c_{1,3}=\frac{h \cdot y_{A}}{I_{x x}} \\
& c_{1,4}=-\frac{h}{I_{x x}} \\
& c_{3,1}=\frac{3}{2 \cdot 2 w \cdot l}+\frac{(3 \cdot 2 w+1.8 \cdot 2 h)}{(2 w)^{2} \cdot(2 h)^{2}} \cdot h \\
& c_{3,5}=-\frac{(3 \cdot 2 w+1.8 \cdot 2 h)}{(2 w)^{2} \cdot(2 h)^{2}} \\
& c_{4,2}=-\frac{h^{2}}{I_{x x}}-\frac{1}{2 w \cdot 2 h} \\
& c_{4,3}=\frac{-h \cdot y_{A}}{I_{x x}} \\
& c_{4,4}=\frac{h}{I_{x x}} \\
& c_{6,1}=\frac{3}{2 \cdot 2 w \cdot l}+\frac{(3 \cdot 2 w+1.8 \cdot 2 h)}{(2 w)^{2} \cdot(2 h)^{2}} \cdot(-h) \\
& c_{6,5}=-\frac{(3 \cdot 2 w+1.8 \cdot 2 h)}{(2 w)^{2} \cdot(2 h)^{2}} \\
& c_{7,1}=\frac{w \cdot y_{A}}{I_{z z}} \\
& c_{7,2}=\frac{w^{2}}{I_{z z}}-\frac{1}{2 w \cdot 2 h} \\
& c_{7,6}=\frac{w}{I_{z z}}
\end{aligned}
$$




$$
\begin{gathered}
c_{9,1}=-y_{A} \cdot \frac{(3 \cdot l+1.8 \cdot 2 w)}{l^{2} \cdot(2 w)^{2}} \\
c_{9,2}=\frac{3}{2 \cdot l \cdot 2 h}-w \cdot \frac{(3 \cdot l+1.8 \cdot 2 w)}{l^{2} \cdot\left(2 w^{2}\right)} \\
c_{9,6}=-\frac{(3 \cdot l+1.8 \cdot 2 w)}{l^{2} \cdot(2 w)^{2}} \\
c_{12,6}=-\frac{(3 \cdot l+1.8 \cdot 2 w)}{l^{2} \cdot(2 w)^{2}} \\
c_{10,1}=\frac{-w \cdot y_{A}}{I_{z z}} \\
c_{10,2}=\frac{w^{2}}{I_{z z}}-\frac{1}{2 w \cdot 2 h} \\
c_{10,6}=-\frac{(3 \cdot l+1.8 \cdot 2 w)}{I_{z z}} \\
l^{2} \cdot(2 w)^{2}
\end{gathered}
$$


The above matrix was further rearranged to group like rows together. To verify if the rows were independent of each other, the cell values had to be further compared. This was achieved by simplifying the expressions with general variables. This allowed a more clear comparison of the individual matrix elements. The simplified matrix variables were defined as,

$$
\begin{gathered}
M=\frac{h}{I_{x x}} \\
H=\frac{1}{2 w \cdot 2 h} \\
N=\frac{w}{I_{z z}} \\
G_{z y}=\frac{(3 \cdot l+1.8 \cdot 2 w)}{l^{2} \cdot(2 w)^{2}} \\
G_{x y}=\frac{(3 \cdot 2 w+1.8 \cdot 2 h)}{(2 w)^{2} \cdot(2 h)^{2}} \\
Q=\frac{3}{2 \cdot l \cdot 2 h} \\
P=\frac{3}{2 \cdot l \cdot 2 w}
\end{gathered}
$$

Substituting these values into the original coefficient matrix and arranging the rows to pair like expressions produced the final matrix, 


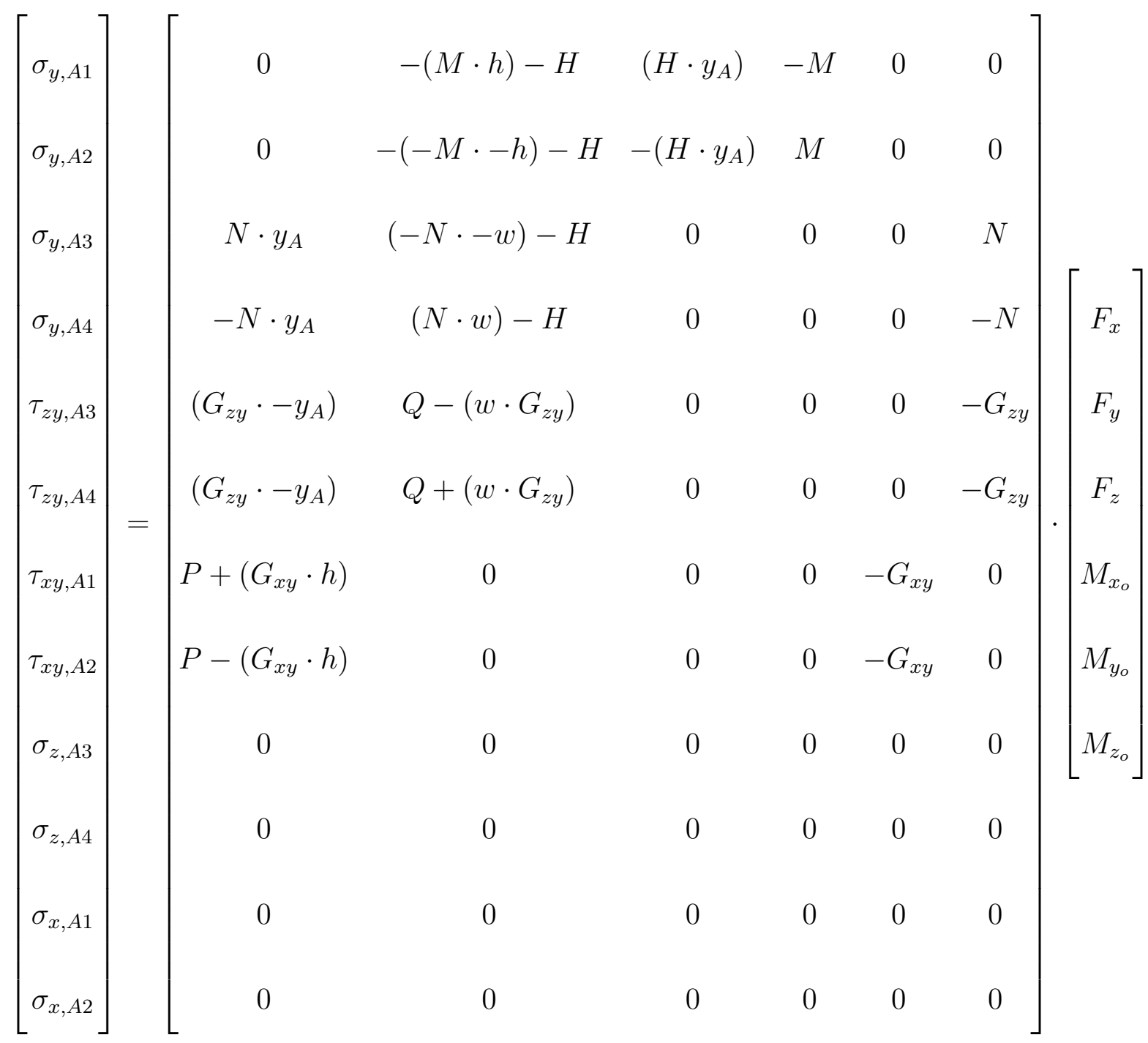

The rearranged matrix of the stresses at Plane $A$ allowed assessment of row independence. The above matrix identified row similarities between the stresses at the symmetric points of $A 1$ with $A 2$ and $A 3$ and $A 4$. This showed 4 pairs of unique rows in the matrix. Comparing these row pairs, it was noted that small differences in variable signs existed, which depending on the magnitude of the changed variable, could cause significant independence between the two rows. A review of the simplified variables, as well as a substitution of placeholder geometric variables into these expressions, confirmed independence. It was evident that these proposed measurement locations would provide row independence to evaluate each unknown input variable. 
A similar analysis can be applied to Plane B. The variation between the two sets of analysis is the position variable of the y-coordinate, $y_{B}$.

\subsubsection{Development of Strain Expressions}

The finalized stress matrix was then applied to produce further expressions to reach the theoretical output of a strain gauge placed on the designed sensing beam. The conversion from theoretical stresses to theoretical strain outputs required further calculations with equations from stress and strain theory. The overall process was outlined previously in Figure 5.5. The following section provides the general equations required to obtain the final strain outputs.

Development of directional stresses acting on Plane $A$ and Plane $B$ was completed in Section 5.2.5 and expressed as a matrix. The next conversion required was that of the directional stresses being further related to the principal stresses ${ }^{1}$ on the planes of interest. The orientation of the principal stresses was also determined. The non-linear relationship which exists between the directional and principal stresses are outlined below, as given by [61, Eq.4.16]. Substitution of the calculated directional $x, y$, and $z$ stresses as well as the shear stress, would provide the values of the principal stress. As the equations display, this process would develop 4 equations across the $x-y$ and $z-y$ planes, for the maximum and minimum stress value on each plane. These equations can be applied across the two positional planes of measurement, Plane $A$ and Plane B. Therefore, when combining all directional stresses across the positional planes, a total of 8 principal stresses would be developed.

$$
\sigma_{1_{x y}}, \sigma_{2_{x y}}=\frac{\sigma_{x}+\sigma_{y}}{2} \pm \sqrt{\tau_{x y}^{2}+\left(\frac{\sigma_{x}-\sigma_{y}}{2}\right)^{2}}
$$

\footnotetext{
${ }^{1}$ The maximum and minimum normal stress values present on an element, in which the shear stress remains at zero.
} 


$$
\begin{aligned}
& 2 \theta_{x y}=\tan ^{-1}\left(\frac{2 \tau_{x y}}{\sigma_{x}-\sigma_{y}}\right) \\
& \sigma_{1_{z y}}, \sigma_{2_{z y}}=\frac{\sigma_{z}+\sigma_{y}}{2} \pm \sqrt{\tau_{z y}^{2}+\left(\frac{\sigma_{z}-\sigma_{y}}{2}\right)^{2}} \\
& 2 \theta_{z y}=\tan ^{-1}\left(\frac{2 \tau_{z y}}{\sigma_{z}-\sigma_{y}}\right)
\end{aligned}
$$

The general expressions for the principal stresses were then related to the principal strains $^{2}$ in the system acting on Plane $A$ and Plane $B$. The theoretical relationship allowed direct conversion from stresses to strains, as given by [61, Eq.5.5], and summarized below. The principal strains are a function of the principal stresses and the material properties of the sensing beam. A total of 4 principal strains would be present at any measurement position on the $x-y$ and $z-y$ planes. Considering the locations of measurement, 4 principal strains would be present at both Plane A and Plane B. Therefore, in consideration of both measurement planes, a total of 8 principal strains were developed. A decrease in total expressions is noted in this step, from moving from directional to principal values.

$$
\begin{aligned}
& \epsilon_{1_{x y}}=\frac{1}{E} \cdot\left(\sigma_{1_{x y}}-\nu \sigma_{2_{x y}}\right) \\
& \epsilon_{2_{x y}}=\frac{1}{E} \cdot\left(\sigma_{2_{x y}}-\nu \sigma_{1_{x y}}\right) \\
& \epsilon_{1_{z y}}=\frac{1}{E} \cdot\left(\sigma_{1_{z y}}-\nu \sigma_{2_{z y}}\right)
\end{aligned}
$$

\footnotetext{
${ }^{2}$ The maximum and minimum normal strain values present on a element, in which the shear strain remains at zero.
} 


$$
\epsilon_{2_{z y}}=\frac{1}{E} \cdot\left(\sigma_{2_{z y}}-\nu \sigma_{1_{z y}}\right)
$$

The principal strains were additionally related to directional strains. This is similar to the relationship between directional stresses and principal stresses. The non-linear relationship is a function of the principal strains and the angle between the determined principal planes, as given in [61, Eq. 5.3,5.4]. Orientations along the defined axes were selected with consideration of the strain gauge orientation options. The initial plan in the gauge selection was to use strain gauge rosettes. This option was advantageous as it would allow three orientations of gauges at one measurement position. Therefore, for this stage of analysis, three orientations of gauges were assessed at each measurement position. This was completed to evaluate orientations of strain at each position of interest. The benefit of each orientation could then be compared further, to complete the final gauge selection. These orientations were expressed as the strain at a $0^{\circ}, 45^{\circ}$, and $90^{\circ}$ orientation. In considering the total number of equations, consideration was therefore made in regards to the orientation positions, the directional planes, and the measurement positions of interest. Considering a measurement position on the $x-y$ plane, the three orientations of interest would develop three expressions. The same would be true when comparing the $z-y$ plane at that measurement position of interest. The strain would be dependent not only on the directional plane, but also its specific measurement position across the beam. Therefore, in assessing the strains at a measurement position such as at Plane A, where four positions of interest for measurement had been selected, a total of 12 expressions would be present. At each of the four measurement positions of interest, 3 expressions would be developed from the orientations. These expressions would be dependent on the directional plane in which the position was within. Finally, in combining the Plane $A$ and Plane $B$ outputs, a total of 24 directional strain expressions would be 
collected.

$$
\begin{gathered}
\epsilon_{0_{x y}^{\circ}}=\frac{\epsilon_{1_{x y}}+\epsilon_{2_{x y}}}{2}+\left(\frac{\epsilon_{1_{x y}}-\epsilon_{2_{x y}}}{2} \cdot \cos 2 \cdot\left(2 \theta_{x y}\right)\right) \\
\epsilon_{45_{x y}^{\circ}}=\frac{\epsilon_{1_{x y}}+\epsilon_{2_{x y}}}{2}+\left(\frac{\epsilon_{1_{x y}}-\epsilon_{2_{x y}}}{2} \cdot \cos 2 \cdot\left(2 \theta_{x y}+45^{\circ}\right)\right) \\
\epsilon_{90_{x y}^{\circ}}=\frac{\epsilon_{1_{x y}}+\epsilon_{2_{x y}}}{2}+\left(\frac{\epsilon_{1_{x y}}-\epsilon_{2_{x y}}}{2} \cdot \cos 2 \cdot\left(2 \theta_{x y}+90^{\circ}\right)\right) \\
\epsilon_{0_{z y}^{\circ}}=\frac{\epsilon_{1_{z y}}+\epsilon_{2_{z y}}}{2}+\left(\frac{\epsilon_{1_{z y}}-\epsilon_{2_{z y}}}{2} \cdot \cos 2 \cdot\left(2 \theta_{z y}\right)\right) \\
\epsilon_{45_{z y}^{\circ}}=\frac{\epsilon_{1_{z y}}+\epsilon_{2_{z y}}}{2}+\left(\frac{\epsilon_{1_{z y}}-\epsilon_{2_{z y}}}{2} \cdot \cos 2 \cdot\left(2 \theta_{z y}+45^{\circ}\right)\right) \\
\epsilon_{90_{z y}^{\circ}}=\frac{\epsilon_{1_{z y}}+\epsilon_{2_{z y}}}{2}+\left(\frac{\epsilon_{1_{z y}}-\epsilon_{2_{z y}}}{2} \cdot \cos 2 \cdot\left(2 \theta_{z y}+90^{\circ}\right)\right)
\end{gathered}
$$

The directional strain equations would provide the direct output from a single strain gauge in the respective position along the plane of interest. However, it was expected in this application that small strain readings would be detected. Therefore, to amplify the reading Wheatstone Bridge circuitry was considered.

\subsubsection{Amplification of Strain through a Wheatstone Bridge}

The ability to accurately measure small changes in gauge resistance is possible with the use of a Wheatstone Bridge circuit. The use of a bridge circuit would increase the sensitivity of the system, which would allow measurable outputs to be recorded [60]. To double the output bridge's sensitivity to strain, a half-bridge circuit could be 


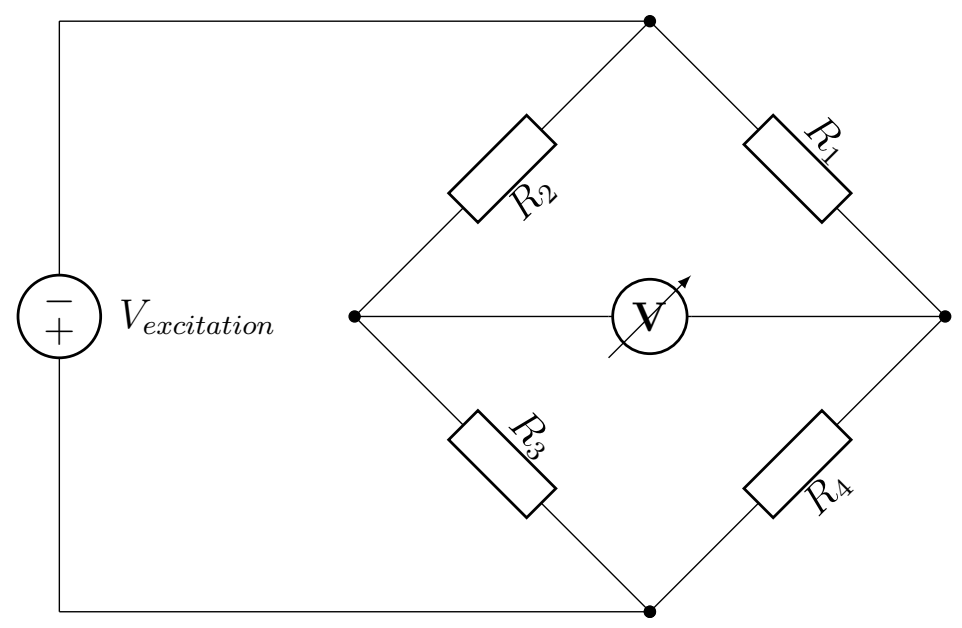

Figure 5.10: General Bridge Circuit

designed. This would require two active gauges per bridge in the system. Application of a half-bridge design provided a total of 12 half-bridge strain outputs. Development of the theoretical bridge equation was completed through reference to [60, Eq. 9.21], as shown in Equation 5.48 and Figure 5.10. The position of the two active gauges in the circuit would be dependent on desired loading application to be measured by the bridge pair; where the other two positions would be that of resistors.

$$
V_{\text {output }}=V_{\text {excitation }} \cdot\left(\frac{R_{1}}{R_{1}+R_{4}}-\frac{R_{2}}{R_{2}+R_{3}}\right)
$$

The resistance values of the active gauges could be theoretically calculated from the gauge's unique gauge factor, nominal resistance, and strain applied at the measurement location; as in Equation 5.49.

$$
R_{\text {bridge }}=\left(G_{F} \cdot \epsilon \cdot R_{\text {nominal }}\right)+R_{\text {nominal }}
$$

The effectiveness of the design, as well as the appropriate selection of gage position and excitation voltage were evaluated through a simulation with the theoretical analysis. 


\subsection{Verification of the Theoretical Analysis}

To further verify the conceptual design, the developed stress analysis approach was applied to the general equations in Section 5.2.7 as outlined in Figure 5.5. The intent of this analysis was to determine quantitatively if the strain gauges would produce measurable outputs. This was critical in the design process to ensure each force created a significant output on the sensing beam to allow each component to be isolated and uniquely solved for. As outlined in Chapter 3.2, minor variations in keystroke are of interest. Therefore, the smallest detectable change from the gauge bridges was verified before finalizing the sensing beam design.

The programming environment $M A T L A B$ was integrated to incorporate the previously defined equations and pass them through the steps outlined in Figure 5.5. The output of theoretical strain values was completed while varying the 6 unknown variables of the keystroke force and position components. This provided a thorough understanding of the critical contact points and the influence the components of force had on the designed system. The script applied to complete this process can be viewed in Appendix D.

The process of completing the theoretical stress to strain conversion required definition of the material and geometry constants as well as the keystroke position and force variables. For the purpose of the verification, nominal variables for the geometry and beam material were input. The theoretical verification process was completed with the selection of an aluminium alloy material. The beam material properties influence the conversion from principal stresses to principal strains, as outlined by Equations $5.38,5.39,5.40$ and 5.41 which depend on the material properties of Young's Modulus and Poisson's Ratio. The relationship of these variables showed that an increase in Young's Modulus would result in a decrease in the principal strain values, and an 
increase in Poisson's Ratio this would also decrease the theoretical principal strain values. The geometric properties of the sensing beam were analyzed under different geometric conditions, to examine the possible extremes of the design. Two of the reviewed geometric designs were that of a solid and hollow square prism. For this verification step, the nominal values of width, height, and length, were set to $7 \mathrm{~mm}$, $7 \mathrm{~mm}$, and $95 \mathrm{~mm}$, respectively.

\subsubsection{Theoretical Strain and Voltage Outputs}

The theoretical strain output was first calculated for each position and orientation of interest, which provided 24 outputs. This was completed for different keystroke cases to compare the effect the input variables had on the system. In summary, 8 loading cases were simulated. These included varying $F_{x}, F_{y}$, and $F_{z}$ along both the $\mathrm{x}$ - and $\mathrm{y}$-axis both separately, and with combined loading cases. This was repeated for a solid and hollow square prism. This simulation confirmed the initial assumptions on the sensitivity of the gauge's orientation and position, with different loading cases. From the first round of simulations it was noted that the strain values could be increased by shifting the sensing beam to a lower z-position in the key. This would increase the $z_{c}$ variable, which would allow an increase in the moment, therefore impacting the final strain values. A comparison between the solid and hollow designs was performed at this position.

First, the range in micro-strain outputs at the position $A 1$, resulting from the variation of $F_{z}$ applied at the front end of the key, was simulated. Figures 5.11 and 5.12 were produced to compare a hollow and solid cross-section, respectively. Comparison of Figures 5.11 and 5.12 display a significant difference in the maximum strain output. A steeper change in micro-strain, noted by the total range, was observed in the simulation for the hollow cross-section. For a gauge positioned to detect $F_{z}$ along 

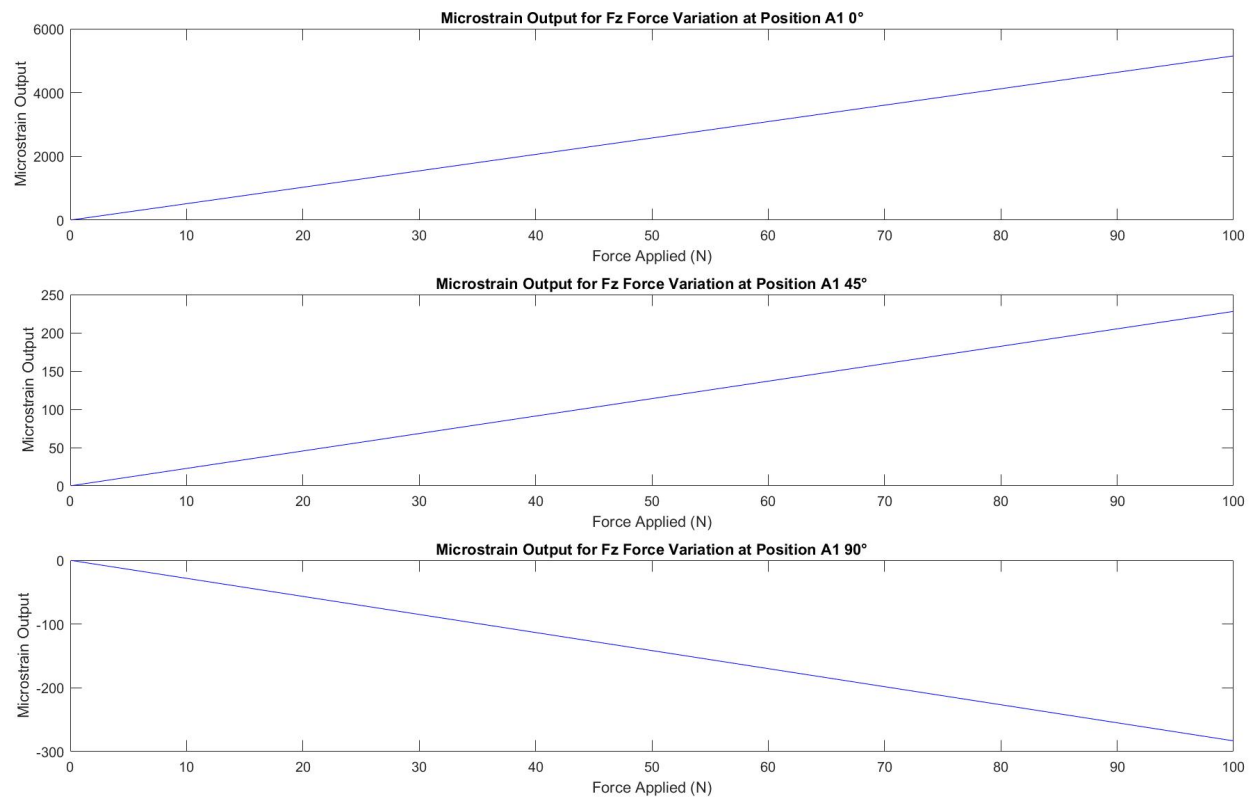

Figure 5.11: Fz Force Variation at A1 for a Touch Position at $(0,0)$ on the Key Surface, for a Hollow Cross-Section
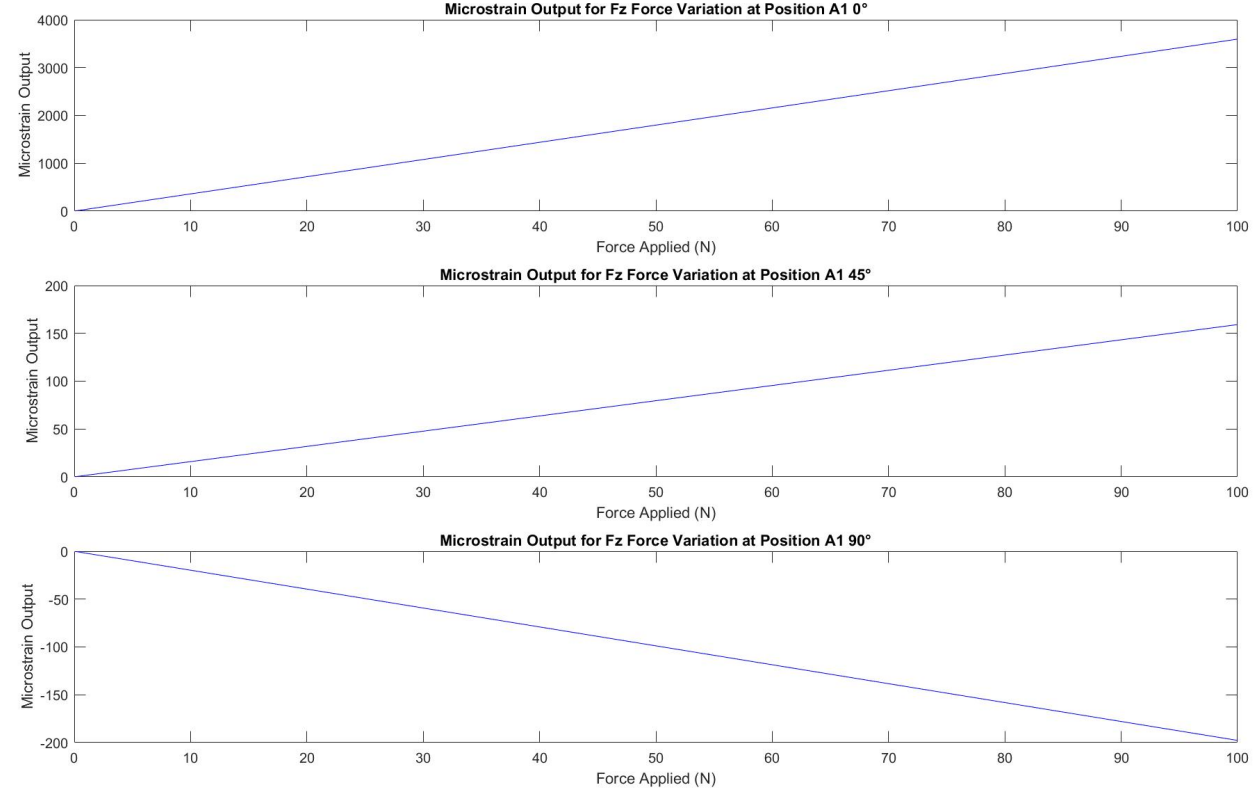

Figure 5.12: Fz Force Variation at A1 for a Touch Position at $(0,0)$ on the Key Surface, for a Solid Cross-Section 
the centreline, the maximum possible strain value would be desired. Therefore, in comparing the outputs in the first graph to the geometry, the hollow design would be more favourable.

Next, the micro-strain output from the previously described $F_{z}$ force variation was assessed at position $B 1$. This was again completed over a hollow and solid cross section, as shown in Figures 5.13 and 5.14, respectively. Comparison of these outputs showed similar results with respect to the difference in cross sectional areas, to those noted at position $A 1$. Comparison was additionally made between the strain outputs at position $A 1$ (Figures 5.11 and 5.12) and $B 1$ (Figures 5.13 and 5.14). Although similarities were noted in the positive and negative responses of the different measurement orientations, the magnitude of the responses were different between the two measurement planes. The overall range in micro-strain was greater across the $A 1$ tests compared to the outputs at $B 1$. These simulations confirmed that the separation of the two planes would impact the overall strain reading. This was expected due to the change in the moment arm. It was noted that the maximum values between the two planes were significantly different. This finding confirmed that the two measurement planes would further allow distinction of the y-position of the applied contact force, as the output values were dependent on the applied moment. Due to the decrease in values when comparing the different beam crosssections, it again was more effective to apply the hollow cross-sectional model. This would allow a greater strain reading along the Plane $B$ values. 

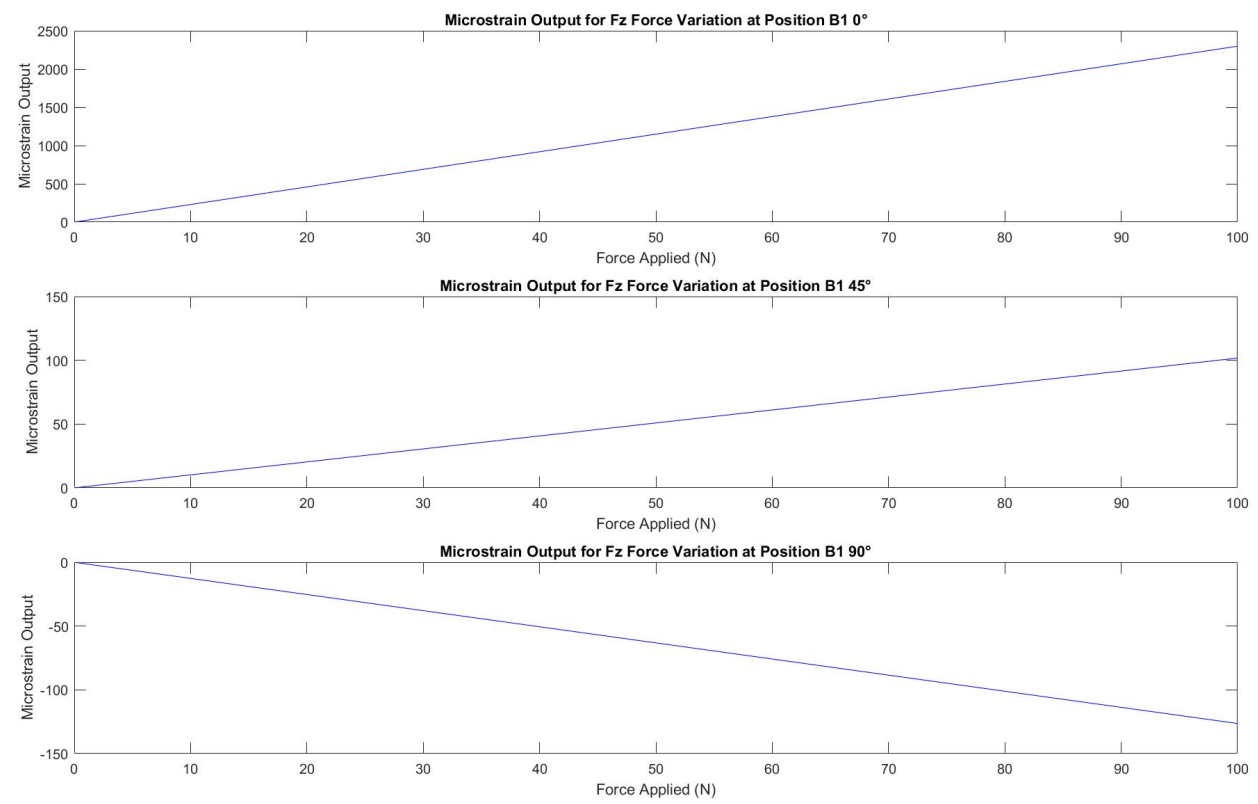

Figure 5.13: Fz Force Variation at B1 for a Touch Position at $(0,0)$ on the Key Surface, for a Hollow Cross-Section
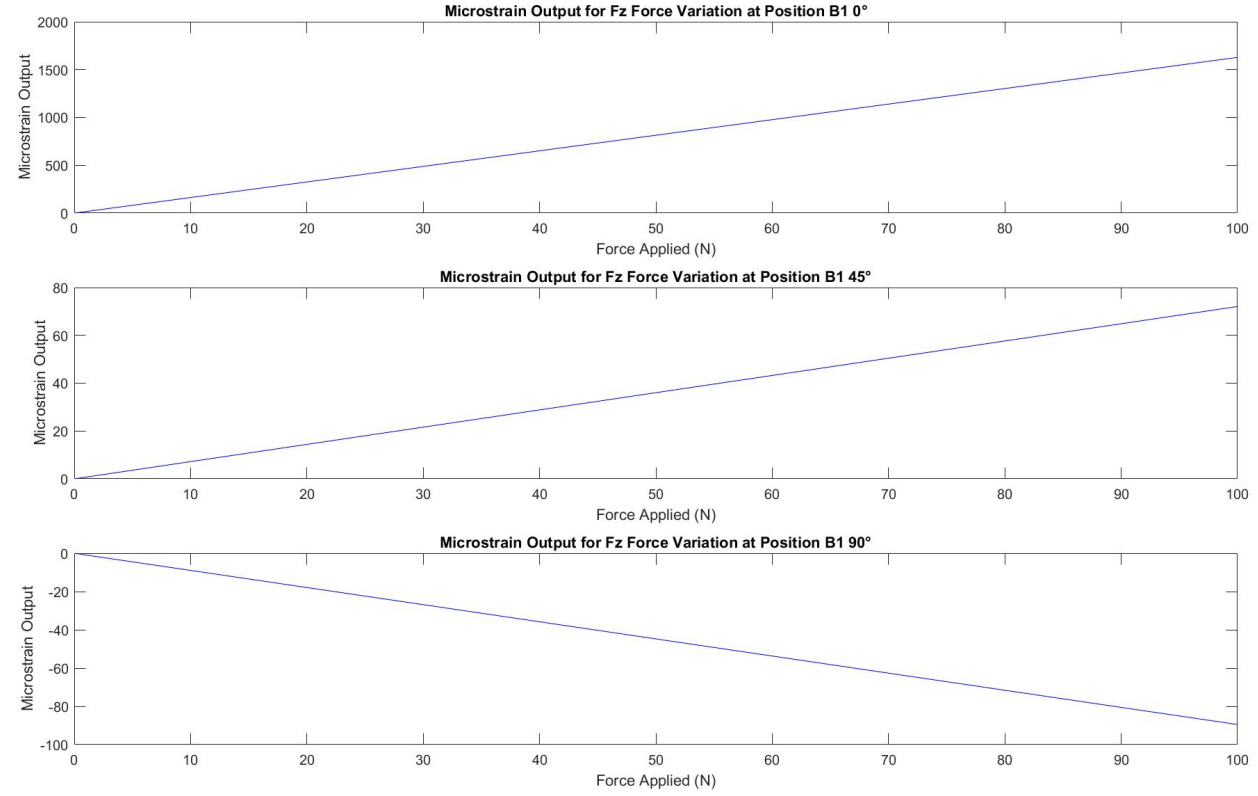

Figure 5.14: Fz Force Variation at B1 for a Touch Position at $(0,0)$ on the Key Surface, for a Solid Cross-Section 
Assessment at the $(0,0)$ origin point along the top key surface would provide the largest moment arm. Therefore, additional simulations varying the y-variable along the top of the key surface were also completed, see Figures 5.15 and 5.16. These simulations provided the response at position $A 1$ and $B 1$ to the variation of an $F_{z}$ force, with the contact position occurring $100 \mathrm{~mm}$ along the y-axis, into the key. This position would be equivalent to a position of touch along the narrow portion of the keytop. Again, these outputs confirmed that variation in the moment arm would directly impact the total micro-strain output. At this varied contact position, the overall range was significantly lower than the previous trials completed. In reviewing the outputs at $B 1$, Figure 5.16, it can be noted that the overall range across the force inputs is much smaller than the other trials completed. These results were for that of a hollow cross-section. As expected, the values were considerably lower in the solid cross-sectional analysis. The lower output range could result in the values being difficult to detect, and distinguish as a voltage against any noise in the system. Therefore, a hollow cross-section would be necessary, provided it can support the applied loads. Three hollow cross-sections were reviewed to compare the estimated stresses and deflections for the expected maximum and maximum overload capacity force applications for $F_{z}$, as it had to highest expected loading. Although a hollow cross-section would experience higher stresses and overall beam deflection when compared to a solid cross-section, the completed analysis confirmed a hollow cross-section would be sufficient under the expected loading cases.

These forms of tests were repeated to vary each loading force. In the $F_{x}$ loading cases, it was critical to compare the gauges placed on the side of the beam to ensure differentiation could be made to identify the x-coordinate of finger touch. It was noted that the maximum strain output was lower than those experienced in the $F_{z}$ loading cases. However, in comparing the outputs of $A 3$ and $A 4$, a distinguishable difference 

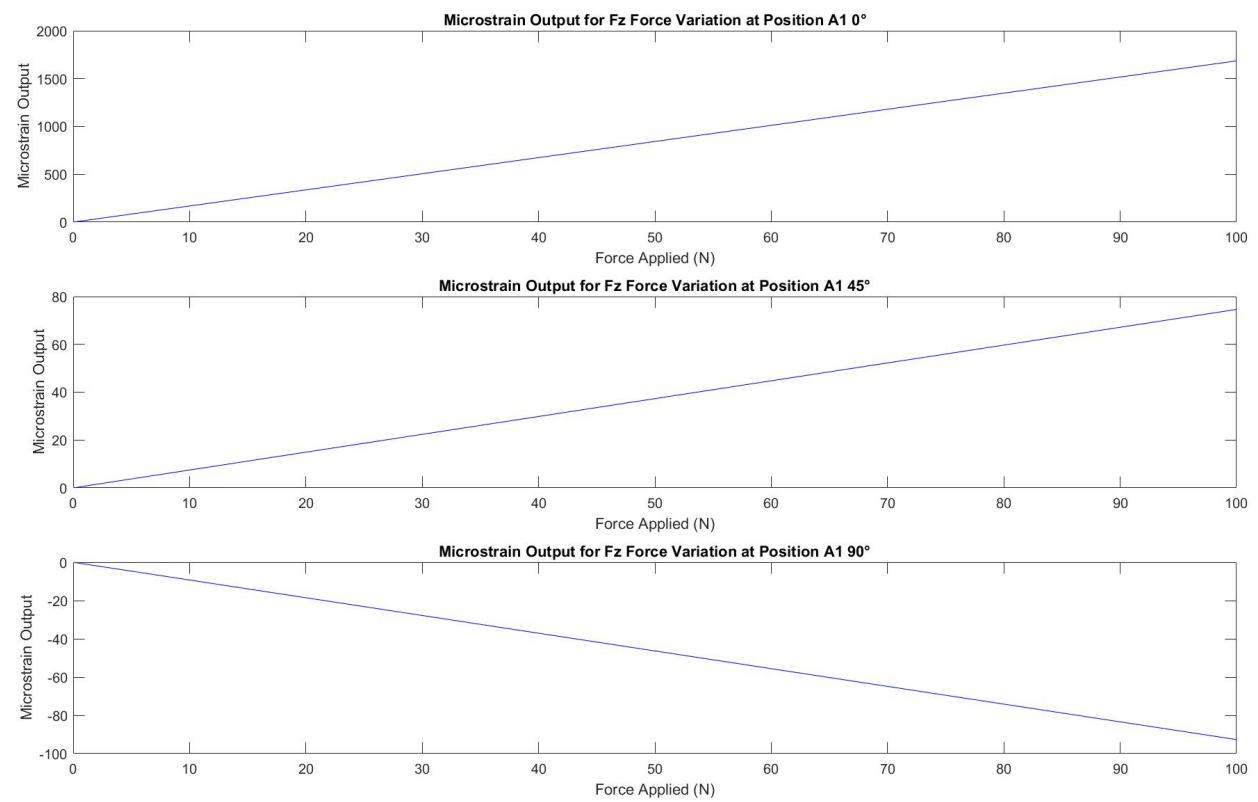

Figure 5.15: Fz Force Variation at A1 for a Touch Position $100 \mathrm{~mm}$ from the y-origin position along the centreline of the Key Surface, for a Hollow Cross-Section
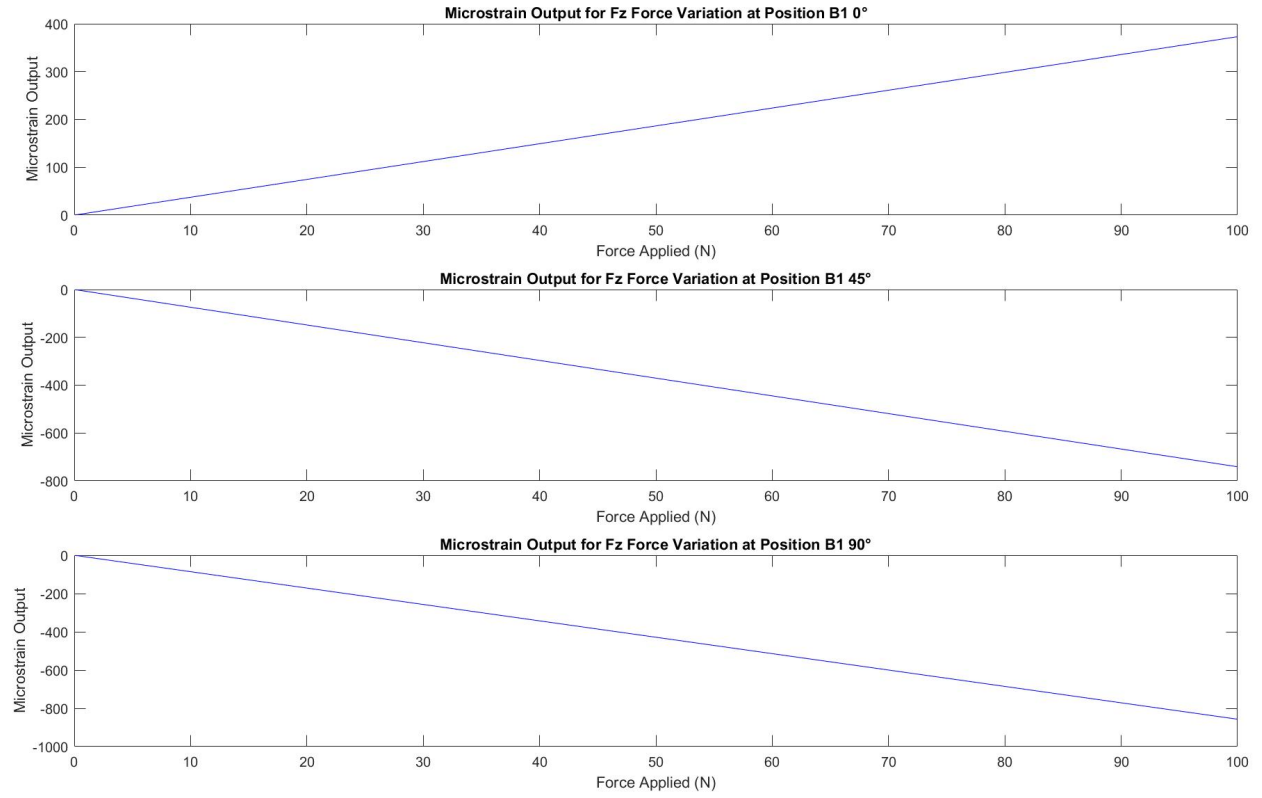

Figure 5.16: Fz Force Variation at B1 for a Touch Position $100 \mathrm{~mm}$ from the y-origin position along the centreline of the Key Surface, for a Hollow Cross-Section 
in output was still noted. This confirmed the effectiveness of these positions. A similar response was noted in comparing the effect of $F_{y}$ loading on the beam. Overall, this initial simulation confirmed the required geometry to maximize the strain output. However, it was still noted from the simulated data that the lower force ranges would produce significantly small strain outputs. Therefore, when assessing the voltage, issues could arise in data processing.

To verify that the theoretical strain values calculated were measurable by the strain gauges and accompanying measurement equipment, the resultant voltage outputs were calculated. As defined in Section 5.2.8, this included the conversion from single outputs to half-bridge outputs. For this analysis, nominal values were input for the circuit. The nominal values of the active gauges and resistors were set to $120 \mathrm{ohm}$, and the gauge factor was set to 2 . These values were selected as they are similar to the true specifications of gauges that fit the size requirements of the system. This was completed through the code included in Appendix D.

The same loading cases described previously were applied to the hollow crosssectional system to compare the minimum and maximum voltage outputs for each half-bridge. This process noted that the maximum values were only on an order of $\mathrm{mV}$. Although it was noted that a small voltage output was to be expected for strain gauge testing, [60, for further theory], it was of concern that the low range would impact the ability to achieve the full desired force range and resolution.

The data processing hardware that was proposed for this application was the $L a b$ Jack Try Pro. At its highest data sampling range it can operate at 16-bits. Dropping, the least significant bit, the maximum and minimum detectable voltage was calculated. Assuming an excitation voltage of $5 \mathrm{~V}$ the minimum and maximum detectable range was $0.15 \mathrm{mV}$ and $5000 \mathrm{mV}$, respectively. For each loading case, the voltage 
output was compared from each bridge to the measurable range. It was evident that for this application, the maximum voltage range would not be reached; however, the minimum readable voltage would cut-off a significant portion of data, much of which was of interest to collect.

To avoid lost data, a gain could be applied to the system. For example, with a $5 \mathrm{~V}$ excitation voltage, a x201 gain could be applied to the system. This would shift the maximum and minimum detectable range to $25 \mathrm{mV}$ and $0.00075 \mathrm{mV}$, respectively. This amplification further encompassed the theoretical voltage outputs. It was noted that the detection of the variables $F_{y}$ and $x$ were the least sensitive to change in the overall output voltage across the bridges of interest. The necessary amplification to meet the resolution requirements were far higher than the other bridges. However, an external amplification could be added to a single bridge to further amplify the final output voltage values.

In assessing each bridge, it was noted that in specific loading cases a bridge may not be detectable even through amplification. However, this was not a sign of a design failure. Instead, this further exemplified the previous analysis which showed that specific gauge orientations would be more sensitive to a loading case compared to others. This was used to further streamline the necessary gauges for an effective analysis. The impact of system noise on these measurements, and the additional amplification, were reviewed in Section 6.3. This allowed final confirmation of the necessary gain and gauge orientations.

\subsubsection{Conclusions and Adaptations}

Overall, the theoretical analysis and simulations proved that the design would meet the measurement speciations outlined in Chapter 3. To develop an optimal design for 
data collection, adaptations were introduced during the simulations. These included physical changes to the sensing beam, strain gauges and positions of interest, and necessary gains required to amplify the bridge outputs.

One adaptation was that of shifting the z-position of the beam, to increase the moment arm. Another physical adaptation was the use of a hollow-cross section. With the low loads being applied to the system, the hollow beam would provide a more sensitive model, while still meeting the structural requirements to withstand loading without failure. The use of aluminium was preferred for similar reasons. The lightweight material would be beneficial for this design, and provide simplicity in mounting strain gauges.

It was originally proposed to assess 8 positions of interest for strain gauge placement. This was proposed for both the ability to measure the 5 unknown variables of force and position, as well as to introduce redundancy in the system to account for measurement uncertainty. However, when completing the simulations, it was noted that positions $B 3$ and $B 4$ did not provide additional useful information in the system. Although they would add a level of redundancy, they would also result in a lower data collection rate due to an increased number of inputs to read. Therefore, in this stage of the prototype, it was concluded that these positions would not be included in measurement.

Finally, to process the voltage inputs, the necessary gains in the system were assessed. In reviewing the data processing hardware, it was noted that a pre-designed maximum gain was that of x1000. It was not possible to confirm this gain value until bench-top testing began, to further assess the noise in the system and environment and its impact on the voltage readings. However, it was noted that this gain may be required to ensure the voltage inputs meet the possible measurement range and 
are able to meet the resolution and sensitivity desired for the system. The initial simulations of voltage outputs proved this would be critical, especially for the shear force detection. 


\section{Chapter 6}

\section{Final Design Assembly}

The following chapter outlines the final manufacturing and assembly details of the proposed design. Section 6.1 provides an overview of the material and geometry selection which was completed after review of the theoretical analysis. Additionally, the placement of the beam with respect to the remainder of the replica piano key was reviewed. Section 6.2 further outlines the manufacturing of the key itself. Finally, Section 6.3 provides the final selection, placement, and data processing decisions made with respect to the strain gauges used for data collection.

\subsection{Beam Assembly}

\subsubsection{Material Selection}

For the purpose of the theoretical analysis, completed in Section 5.3, aluminium was selected as the beam material. In reference to the predicted strain values, the use of aluminium was a satisfactory selection, as the calculated strain values were in a readable range to be used in data collection. The other main factor considered for material selection was the mass addition from the beam to the key system. A lightweight material would be preferable to ensure that a high mass concentration was 
not present at the front of the piano key. Therefore, aluminium or a 3D printed plastic material would be beneficial. These two options would provide sufficient strength against the loads applied, while carrying a low density to meet weight restrictions. However, in comparing a composite 3D printed material with aluminium, the latter would provide ease of strain gauge mounting. Therefore, the final prototype was completed with an aluminium sensing beam.

\subsubsection{Geometry Selection}

Section 5.3 completed a geometry comparison of a solid and hollow sensing beam. It was noted that a hollow beam would result in significantly higher strain outputs, which was desired for this application. Therefore, the sensing beam was selected as a hollow rectangular prism. The outer geometry of the beam was restricted by the geometry of the piano key. As outlined in the conceptual design, the beam was intended to be placed within the key's geometry to not interfere with adjacent keys. An optimal design to maximize the strain output would require the beam to have a minimum outer geometry. However, the geometry would still need to support the predicted loads as well as provide ample room for strain gauge mounting. In the theoretical stage of analysis, various size constraints were assessed to predict their outcomes. Review of commercially-available geometries within these specifications provided the final geometry. It was concluded with consideration of the above-mentioned factors, the $7 \mathrm{~mm}$ width and height constraints used in the final analysis were preferable for the beam geometry. The final beam selected was a hollow square cross-section with an outer measurement of $6.35 \mathrm{~mm}$ (approximately $1 / 4 \mathrm{in}$ ) with a wall thickness of $1.6 \mathrm{~mm}$ (approximately $1 / 16 \mathrm{in}$ ).

Selection of the beam total length would also greatly impact the ability to complete measurements. To optimize the differentiation between Plane $A$ and Plane $B$ strain 
readings, the maximum span between the planes would be required. Again, the limiting factor in this design was the geometry of the key. One element of this geometry was that of the front and middle balance rail holes present in the key, see Figure 6.1. In replicating the piano key, these portions were to remain unchanged to ensure obstructions to key movement were not present. The other consideration of the geometry was that of the outer geometry. Variation is visible to the pianist in the form of the white key fronts which change in shape within an octave. Additionally, in moving across a keyboard, the angle of alignment between the back and front of the full key varies, see Figure 6.2. To create a design which would be reproducible across multiple keys, the changing key angle should not interfere with the sensing beam. Therefore, the length of the beam was constricted to the front of the key, before the key bend and within the balance rail holes.

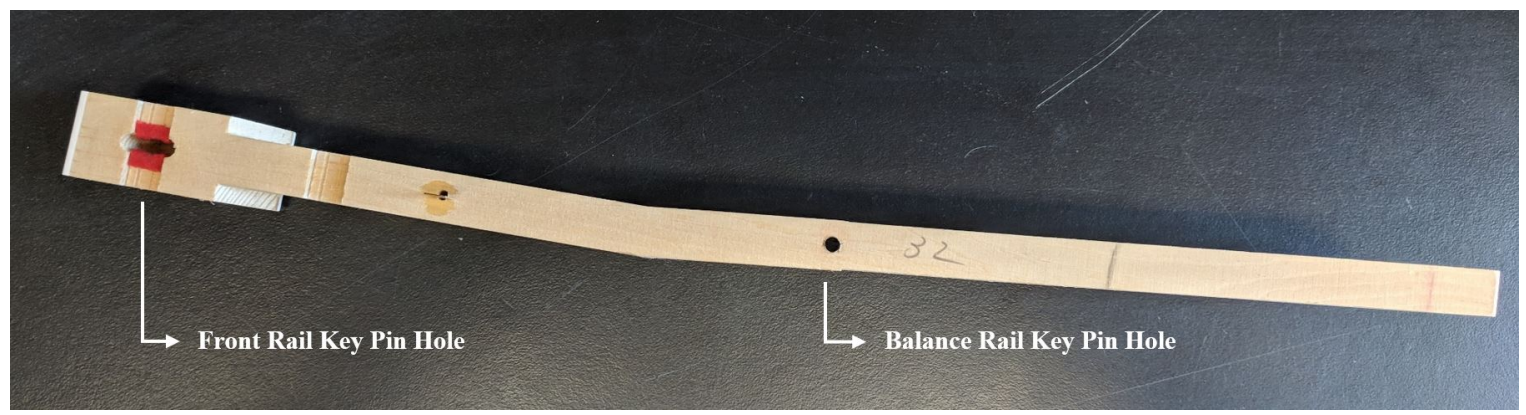

Figure 6.1: Bottom View of Piano Key, with a Front and Middle Balance Rail Hole

The final consideration of the sensing beam geometry was the attachment of the beam to the remainder of the replica piano key. It was intended that the attachment provide a non-permanent solution, to allow for future adjustments to the sensing beam to be possible without altering the remainder of the key prototype. Multiple design iterations were completed to develop the final beam attachment. Designs included kinematic coupling methods as well as slotted connection and bolt designs, similar to those outlined in Figure 6.3. These were considered as they would provide 


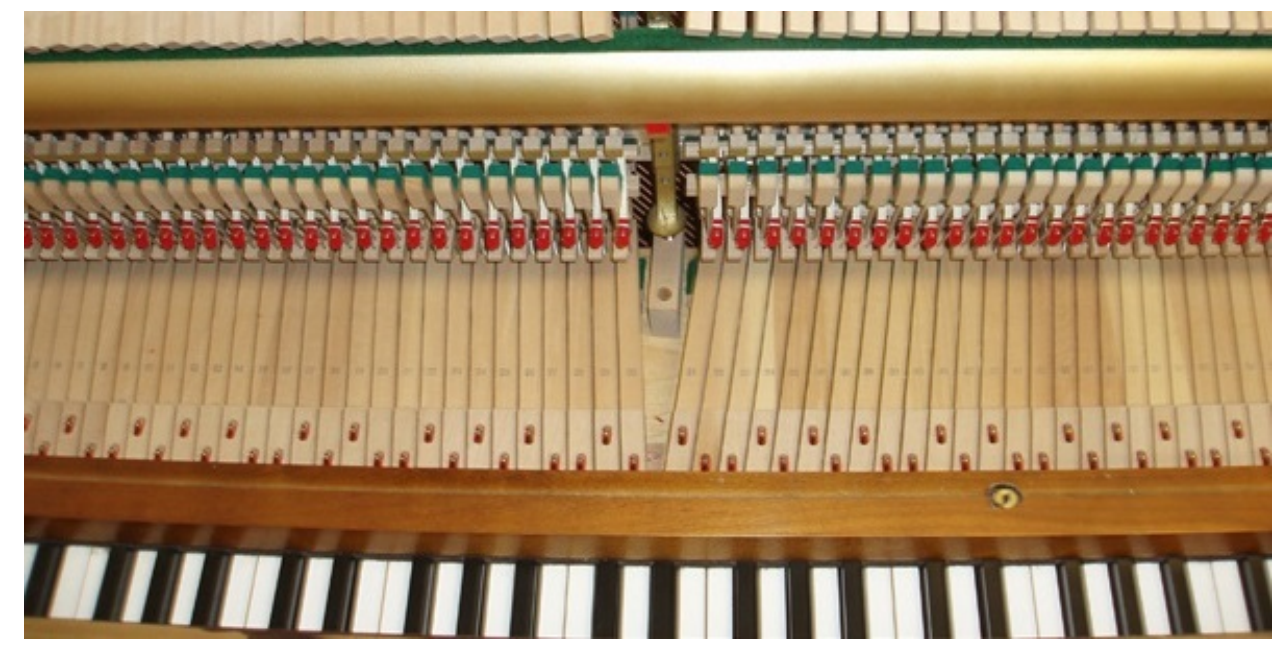

Figure 6.2: Key Angle Variation Across a Keyboard [4]

a constrained system. However, these structural interfaces would require a more involved machining process with a high degree of accuracy. In assessing these design options, it was noted that a more complicated design may result in a less reliable, and reproducible, attachment. As outlined in the principle of Occam's Razor, the simplest solution is often more effective. Therefore, a simplified pin and bolt design was selected.
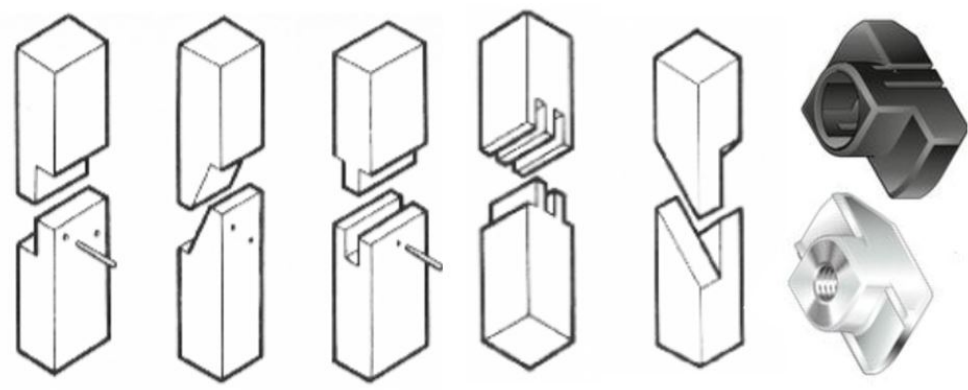

Figure 6.3: Example of Connection Types for the Sensing Beam [5, 6, edited]

Bolted designs are beneficial as they provide a relatively simple design, and are easy to disassemble. Addition of a dowel pin further allowed alignment adjustments 
to be made. The pin and bolt combined attachment did constrain relative movement of the parts. To put this design into practice with the sensing beam, attachment plugs were manufactured for the front and back fo the beam. These plate designs were then secured at either end with the pin and bolt. The plate plugs were affixed to the sensing beam with an epoxy. The alignment and final assembly can be viewed in Figure 6.4.

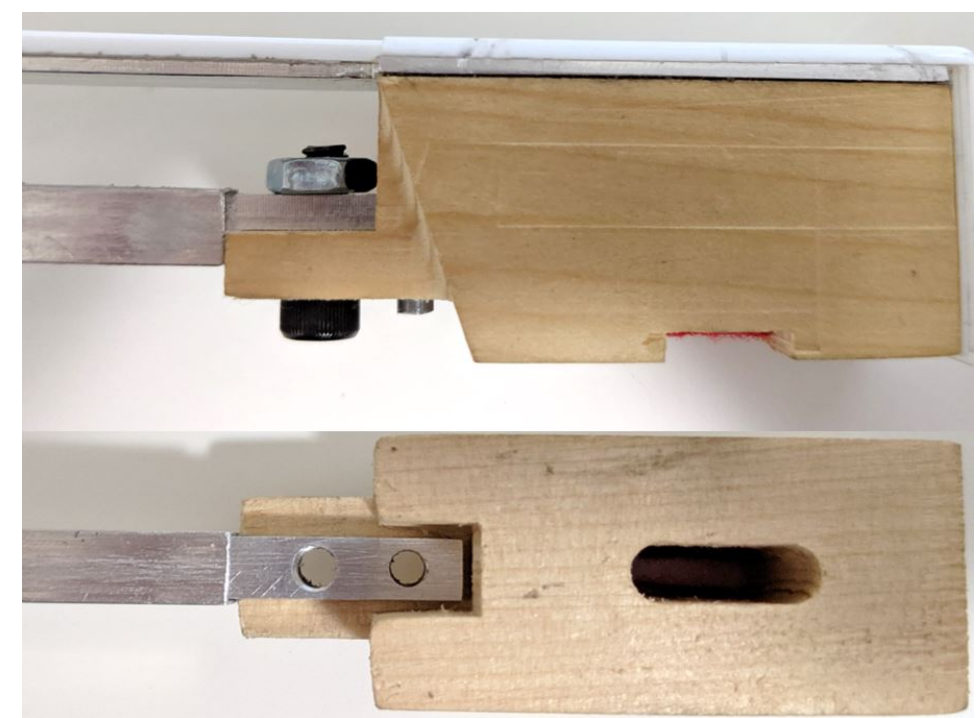

Figure 6.4: Sensing Beam Attachment and Alignment of Pin and Bolt Hole

\subsection{Key Assembly}

Upon completion of the sensing beam initial design, final design and manufacturing decisions were required for the remainder of the piano key. Section 3.2.1 outlined that a 3D scan of the original key was completed to obtain the detailed geometry, see Appendix A for further details. However, this was also completed to provide a model of the key for further editing. The scanned STL file was referenced to create the key model. This model was further edited to remove the material necessary to add the sensing beam and keytop. The final drawings used for manufacturing can be viewed 
in Appendix E.

The material for the remainder of the prototype key was selected to be comparable to that of the original piano key. Piano service manuals have cited various wood sources for the body of the piano key, for example sugar pine and Japanese spruce [1]. An available wood source of similar density and hardness, yellow cedar, was selected for the current prototype. Manufacturing was completed through CNC and manual milling to obtain the final design. Felt was additionally applied to the front and middle balance pin holes to replicate the original key. A plastic white keytop was affixed to an aluminium support which was added to the front of the wooden key prototype. The aluminum support was necessary to reduce the overall deflection of the white keytop. The connection between the plastic keytop, the support, and the wooden keyfront was made semi-permanent. This would allow potential for different keytop options to be assessed, required edits to the front portion of the key to be made, and allow an experimental keytop to be used for validation testing. The layers were affixed through a double-sided adhesive. As the keytop would experience the greatest loading through the vertical force application, therefore further compressing the adhesive, this solution was sufficient for the expected loads. The keytop did not make a connection with the back portion of the piano key, as shown in Figure 6.5. This design decision was critical to ensure all loads were transferred to the sensing beam.

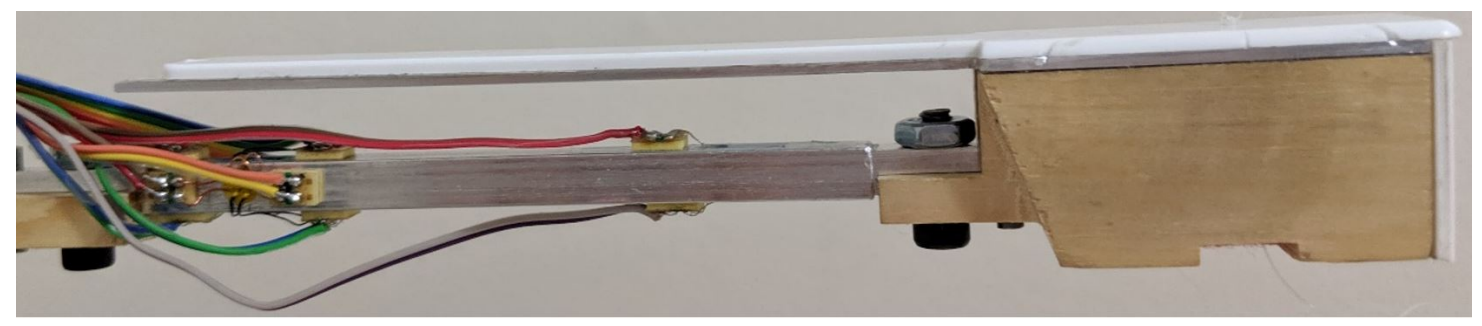

Figure 6.5: Keytop Connection to the front of the Prototype Key 
The final assembly of the key required correct alignment to achieve the unique angle of the key being replicated. An alignment rig was setup to meet the angle requirement for the specific key; in which the original angle was previously measured, see Figure 6.6. With the pin inserted in the back sensing beam attachment, the angle of alignment was adjusted until it met the measured angle. At that point, the key could be fully assembled in place. The full assembly of the manufactured components of the prototype key, without the keytop addition, are shown within the alignment rig in Figure 6.7.

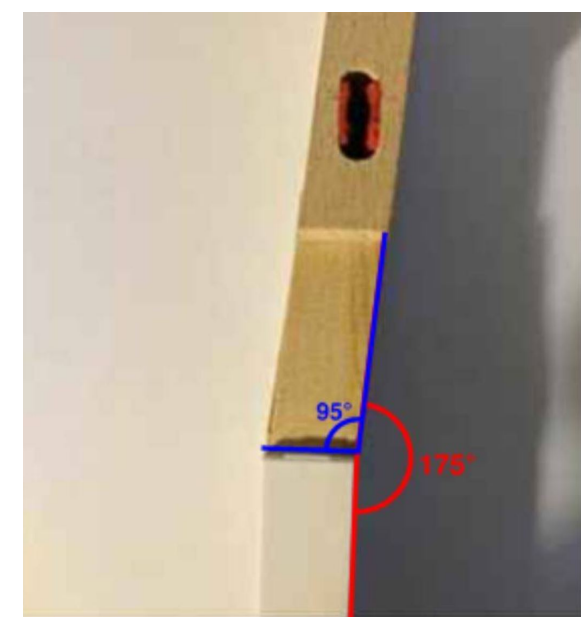

Figure 6.6: Measured Angle for the Key 42 Prototype

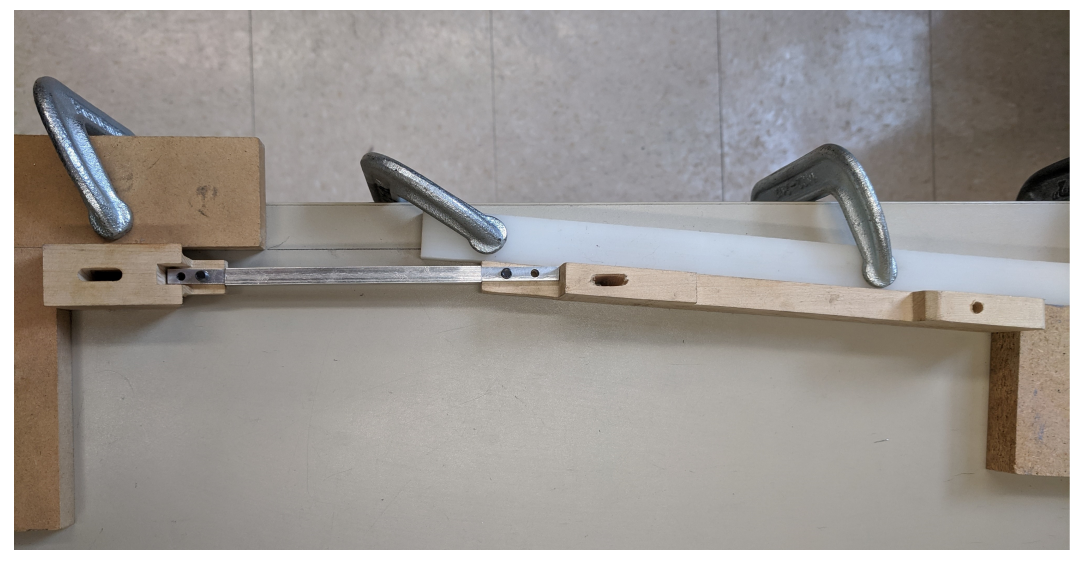

Figure 6.7: Alignment of the Manufactured Components of the Prototype Key 


\subsection{Electronics Assembly}

\subsubsection{Strain Gauge Selection}

As Chapter 5 outlined, strain gauge rosettes were considered for the final design. This was proposed as complex loading cases were expected from the unique pianist keystroke interactions. However, the stress and strain analysis provided further insight on the theoretical strains. Table 6.1 visually summarizes the theoretical stress responses from each gauge orientation. The strength of the response to each contact variable was categorizes as strong (green cell), moderate (yellow cell), and weak (red cell). It had been previously determined that positions $B 3$ and $B 4$ would not be utilized in the current prototype. Although it would provide further redundancy to the system, it did not provide new information and would result in a lower sampling speed. Therefore, the positions $A 1-A 4$ and $B 1$ and $B 2$ were retained. The strong and moderate responses were evaluated to determine which orientations would provide the strongest collection of gauge responses to detect the 5 unknown contact variables.

The orientations at bridge positions $A 12$ showed a strong response to $F_{z}$ and yposition, and a moderate response of $F_{y}$ at the $0^{\circ}$ orientation. Moderate to weak responses were noted across the other orientations at this pair. Therefore, they may not provide signifiant information in this analysis. Although they would provide redundancy in measurement, the low responses would cause difficulty to differentiate between the different variable responses and the noise in the system. The pair $A 34$ only noted a strong response from $F_{x}$ at the $0^{\circ}$ orientation, which would have corresponded to applied bending. A moderate response to $F_{y}$ and the x-position was also present at this orientation. To further establish a response from the weak channels, an additional channel would be beneficial. A similar response was noted between 
the two remaining orientations at $A 34$. A detection of shear strains was noted to be effective for the sides of the key through the use of gauges positioned at $45^{\circ}$.

Table 6.1: Strength of Gauge Orientation Responses

\begin{tabular}{|c|c|l|l|l|l|l|}
\hline Gauge Pair & Orientation $\left(^{\circ}\right)$ & $F_{x}$ & $F_{y}$ & $F_{z}$ & $M_{x}(\mathbf{y})$ & $M_{y}(\mathbf{x})$ \\
\hline$A 12$ & 0 & & & & & \\
\hline$A 12$ & 45 & & & & & \\
\hline$A 12$ & 90 & & & & & \\
\hline$A 34$ & 0 & & & & & \\
\hline$A 34$ & 45 & & & & & \\
\hline$A 34$ & 0 & & & & & \\
\hline$B 12$ & 45 & & & & & \\
\hline$B 12$ & 90 & & & & & \\
\hline$B 12$ & 0 & & & & & \\
\hline$B 34$ & 90 & & & & & \\
\hline$B 34$ & $B 5$ & & & & & \\
\hline$B 34$ & & & & & & \\
\hline
\end{tabular}

The evaluations allowed final gauge confirmation. On the top and bottom of the key, a linear gauge pair could be used at both positions $A 12$ and B12. This would allow strong responses to $F_{z}$ and the y-position, as well as a moderate response to the axial loading of $F_{y}$. It was more effective to limit detection to that of these loads through the use of linear gauges. On the sides of the key at $A 34$ a rosette would be efficient. As the responses to $F_{x}, F_{y}$, and x-position were at a lower magnitude, it was important to use multiple orientations to produce a final reading. Therefore, a combination of linear gauges and rosettes was used. 
For this application, N11-FA-5-120 strain gauges from Showa Instrument were selected for the linear gauges. The nominal resistance of this gauge model is $120 \mathrm{ohm}$, with a gauge factor of 2 , and its overall base dimensions for the length and width are $9.5 \mathrm{~mm}$ by $3.5 \mathrm{~mm}$, respectively. Additionally, this model is manufactured with wired leads to provide ease of soldering. These gauges were selected for positions $A 1, A 2, B 1$, and $B 2$ with the intention to detect $F_{z}$ and y-position.

The proposed measurement of $F_{y}$ and x-position could be measured by a gauge placed along the side of the beam parallel to the sensing beam, in positions $A 3$ and $A 4$. However, detection of shear forces from $F_{x}$ would require gauges to be arranged for shear measurement. To achieve both of these requirements within the size constraints, the proposed use of rosettes would be most efficient. A variety of commercial rosettes were reviewed. Due to the limited gauge mounting area, it was concluded that a stacked rosette would be applied. RF93-0.8/120ZE gauges (with lead wires) from $H B M$ were selected, see Figure 6.8. The nominal resistance of each gauge is 120 ohm, with an approximate gauges factor of 2, and their overall base dimension is $5 \mathrm{~mm}$ in diameter. Stacked rosettes allow strain measurement in confined areas. However, their disadvantage lies in that the stacked components become more sensitive to temperature differences [60]. As use of the prototype was intended to be completed in a controlled temperature environment, as would be expected with any professional musical instruments, large temperature fluctuations which could negatively impact the gauges were not considered a potential issue. However, the sensitivity to temperature would limit the maximum excitation voltage which could be applied to the gauge. A high voltage input would produce thermal effects due to the current flowing through the gauge, which could cause permanent damage to the rosettes. Therefore, upon manufacturer recommendation, a maximum excitation voltage of $3 \mathrm{~V}$ across each bridge was applied. 


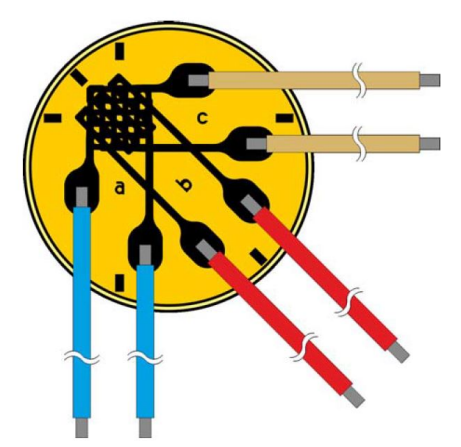

Figure 6.8: RF9 Stacked Strain Gauge Rosette [7]

Strain gauges with pre-wired leads were selected to ensure gauges were not damaged during wire soldering. To further protect the gauges, solder relief terminals were additionally acquired. Solder terminals allow installation between the strain gauges and the connecting cables to the bridge electronics. These were selected to provide ease in soldering, provide strain relief to the connections, and to provide a failure point. In the event of mechanical failure of the cables connecting to the bridge circuitry, solder terminals could be replaced without impacting the gauges.

\subsubsection{Strain Gauge Mounting and Bridge Assembly}

Gauge positions were previously outlined in Section 5.1.3. The position of Plane $A$ and Plane $B$ were further defined with reference to Saint-Venant's Principle. In placing the gauges, consideration of the stress concentrations at the ends of the beam were made. The principle defines that stress concentrations at points sufficiently far from the point of load application are not impacted the stresses produced within the body of the object. To avoid gauge reading error due to stress concentrations produced at the ends of the beam, it was noted that the planes were to be aligned at a point sufficiently far from the point of load application. Therefore, gauge Planes were positioned a distance of at least one beam width away from the beam end. This 
ensured strain readings were not a factor of an increased stress concentration [61, for further reference]. Additionally, as the final sensing beam would be calibrated, any small stress concentrations in the beam would not cause a significant impact on the measurements. Consideration of this principle provided final gauge positioning. Finally, the alignment of the gauges was considered. As previously discussed, the rosettes were acquired to measure shear forces applied during the complex loading of a keystroke. To maximize data recording, the rosettes were arranged to allow one gauge to lie in a $0^{\circ}$ position along the neutral axis while the two remaining gauges were aligned in a $45^{\circ}$ position, see Figure 6.9.

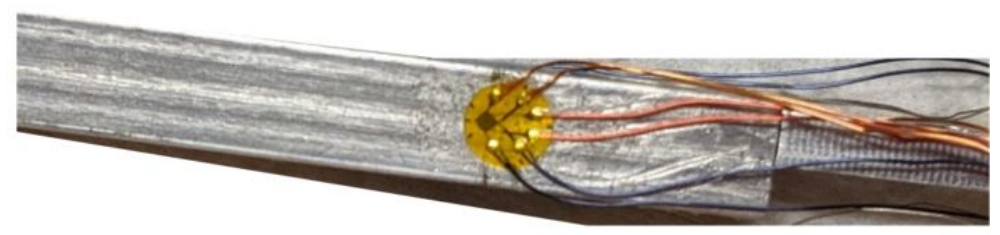

Figure 6.9: RF9 Rosette Mounted to the Sensing Beam

Placement of gauges on the sensing beam was completed following standard strain gauge mounting practices. A microscope was used to further improve the accuracy in gauge alignment during mounting. A protective coating of acrylic lacquer was further applied to the gauges to protect against moisture and contaminants that would negatively impact the gauge stability. Lead wires were additionally soldered to the strain relief terminals, to which ribbon cables were soldered to the terminal pairs to further extend to the circuitry board. This arrangement is shown in Figure 6.10.

The wire pathway of the gauges from the strain terminals were then defined. Due to the sensing beam location, all ribbon cables from the strain relief terminals began at the front half of the piano key. These cables were required to attach to the circuitry 


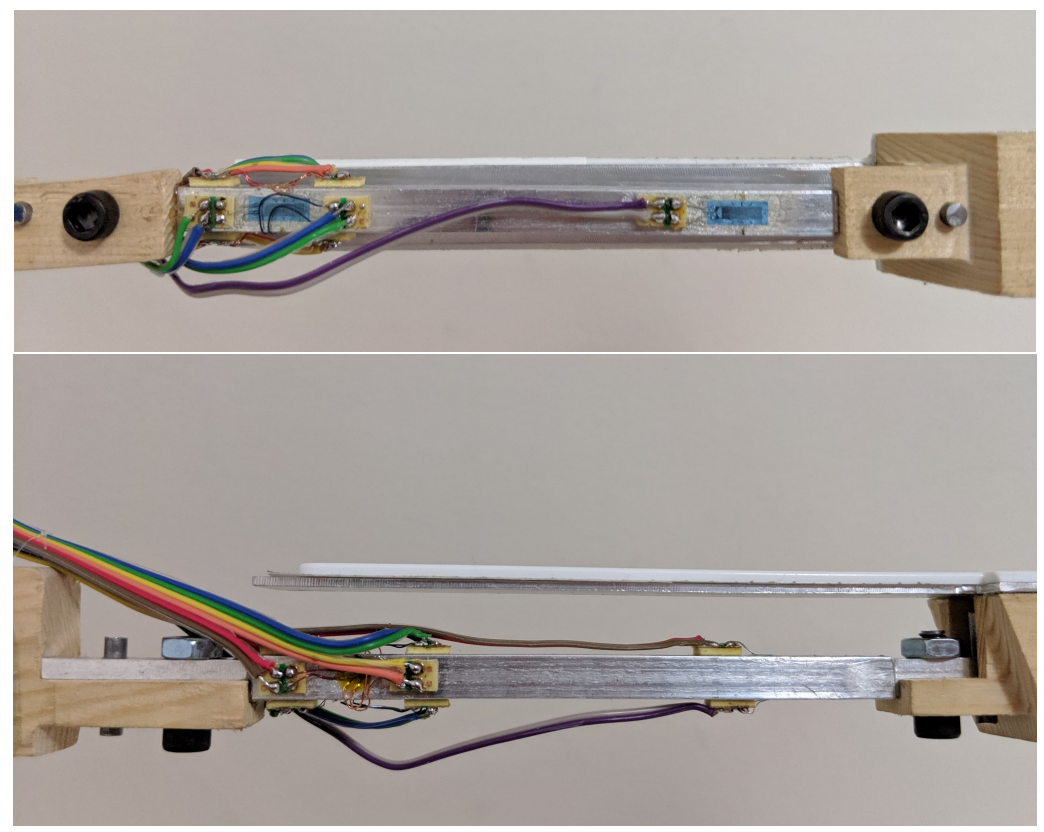

Figure 6.10: Side and Bottom View of Mounted Gauges

board. Therefore, assessment of the board location within the piano keyboard was necessary to define the wire pathway. To reduce electrical noise within the system and wire resistance, it was critical to place the board circuitry at a close proximity to the strain gauges. To reduce the noise in the system, circuit board locations proximal to the prototype key positions were reviewed. Pianos with a MIDI bar, such as the model used for the prototype piano key, have limited space under the front half of the keyboard. As shown in Figure 6.11, space for circuity placement would only be possible under the keyboard at the back half of the key, or above the keys themselves. Placement of circuitry above the keys, behind the piano front panel, would not be visible to pianists. However, it could require further temporary modifications to be made to the piano. A less obtrusive alternative would be to place the circuitry under the piano keys of the keyboard, situated at the back half of the key behind the balance rail. Ample space, both for the layout of the circuitry and vertical height during key depression, were present in this position. 


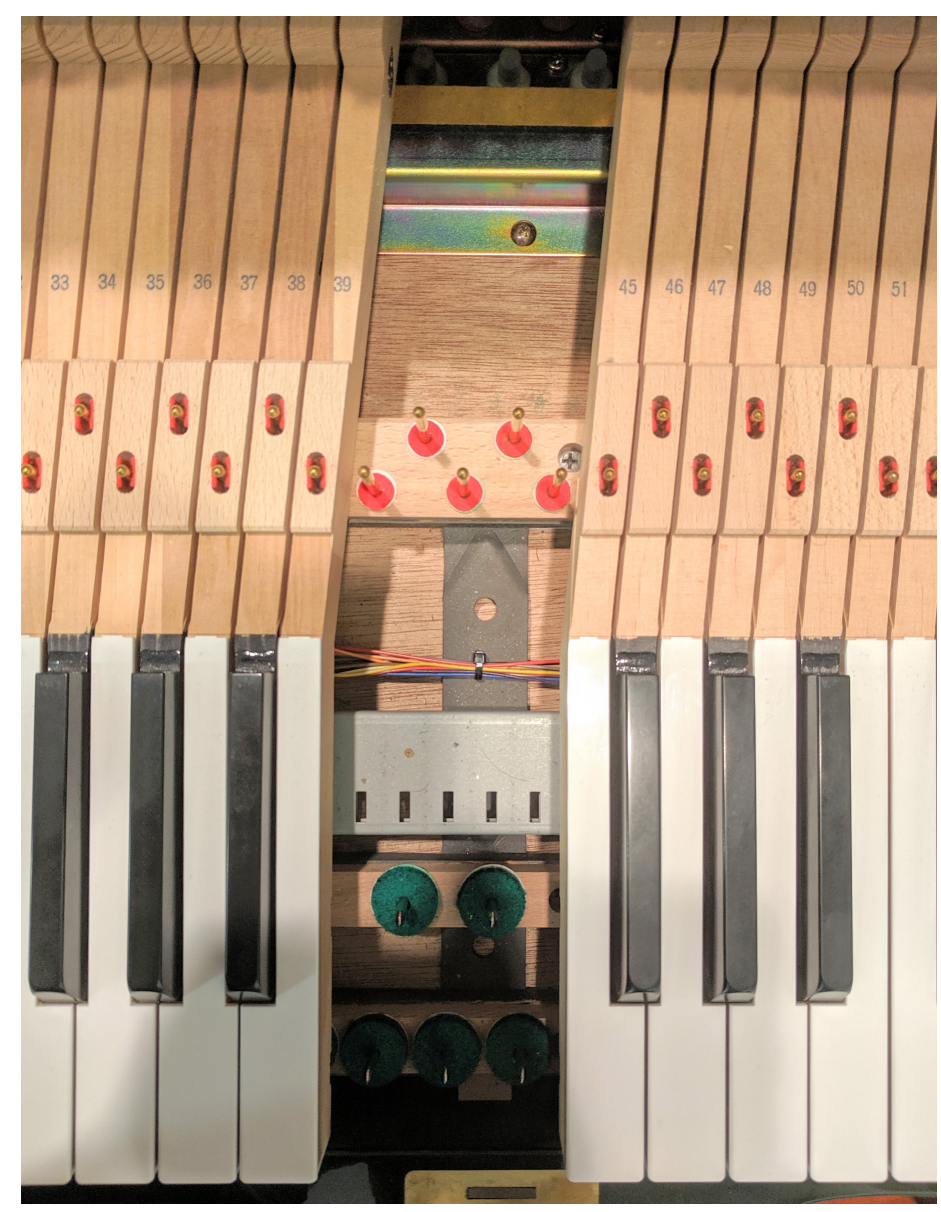

Figure 6.11: MIDI Bar Placement Beneath Upright Piano Keyboard

The wire pathway was required to reach the back of the keyboard. Therefore, wires would be required to wrap around the key, or thread through the keys. To avoid interference with adjacent keys, the latter design was selected. As displayed in Figure 6.12, through holes were placed to thread the wires over the balance rail and down to the circuitry below the key. The cables were further secured in place using fishing line, to limit cable movement between their attachment to the bridge circuitry. These steps were additionally put in place to minimize a second loading path, due to the bending or moment on a wire. 


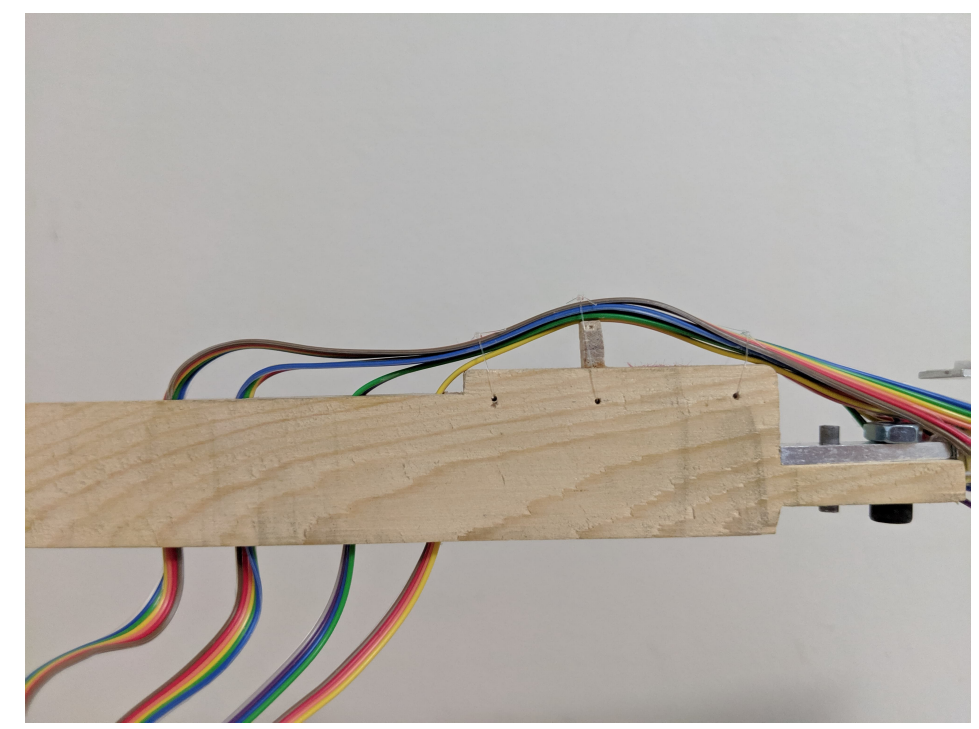

Figure 6.12: Wire Pathway of Ribbon Cables

Section 5.2.8 summarized the use of Wheatstone half-bridge designs to improve the measurable output from the sensing beam and compensate for temperature sensitivity. The half-bridge would require the pairing of two active gauges with two resistors to complete the circuit. $120 \mathrm{ohm}$ resistors were selected for the remaining circuit components. Determination of gauge pairs was made with reference to the expected loading cases. Gauges could be arranged in one of two configurations, that to measure readings in the same, or opposing directions, see Figure 6.13. As the bridge measures the differential voltage, these arrangements would in turn double the output voltage reading. Therefore, to measure axial loading conditions the former configuration would be beneficial, while the latter would be preferred for bending and shear loading conditions. In reference to the prototype, the final bridge pairings were made, see below.

- A1 linear gauge and $A 2$ linear gauge (bridge design 1)

- B1 linear gauge and B2 linear gauge (bridge design 1)

- $A 3$ rosette $0^{\circ}$ gauge and $A 4$ rosette $0^{\circ}$ gauge (bridge design 2) 

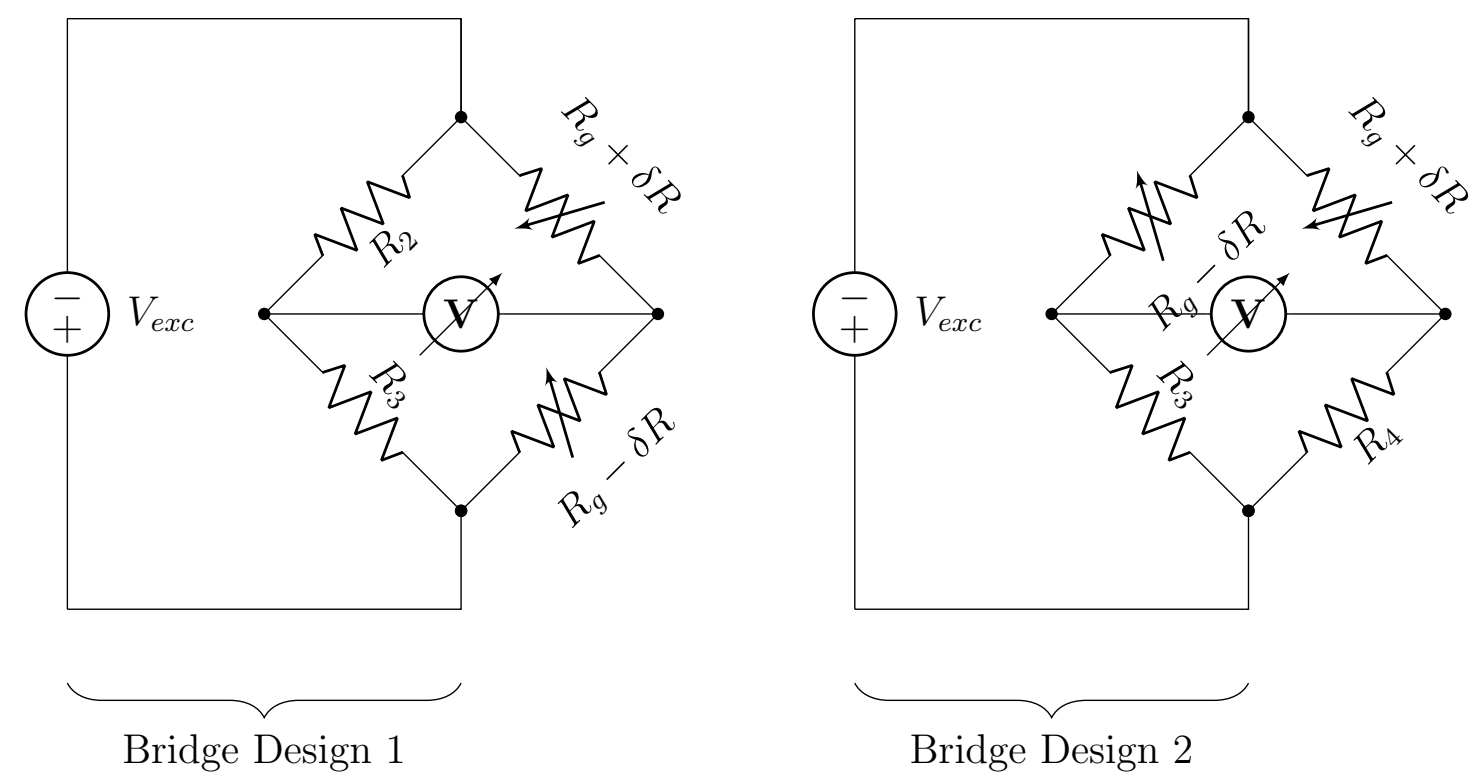

Figure 6.13: Wheatstone Half-Bridge Configurations for Bending/Shear Loading and Axial Loading, Respectively

- $A 3$ rosette $+45^{\circ}$ gauge and $A 4$ rosette $+45^{\circ}$ gauge (bridge design 1 )

- $A 3$ rosette $-45^{\circ}$ gauge and $A 4$ rosette $-45^{\circ}$ gauge (bridge design 1 )

The final bridge schematics were then transferred to a breadboard. In the first iteration of prototyping, a solderless breadboard was utilized. Upon confirmation of circuitry, the design was moved to a perfboard. A perfboard, in which circuitry was soldered, provided a more robust connection. To allow ease of assembly, terminal blocks were utilized between the board and gauge terminal cables. Two perfboards were acquired to allow arrangement of the linear and rosette gauges on separate boards. This was completed to provide ease of verification as well as potential to provide two separate excitation voltages, to the two different gauge styles. The completed assembly, shown in Figure 6.14, provided a streamlined model which could be placed along the keybed under the adjacent piano keys to the prototype. Wiring from the circuit board was then directed to the data acquisition system to process 
the strain data. Ribbon cables were selected for the input and output circuitry at the perfboard. This allowed further organization of wiring, and a more compact design.

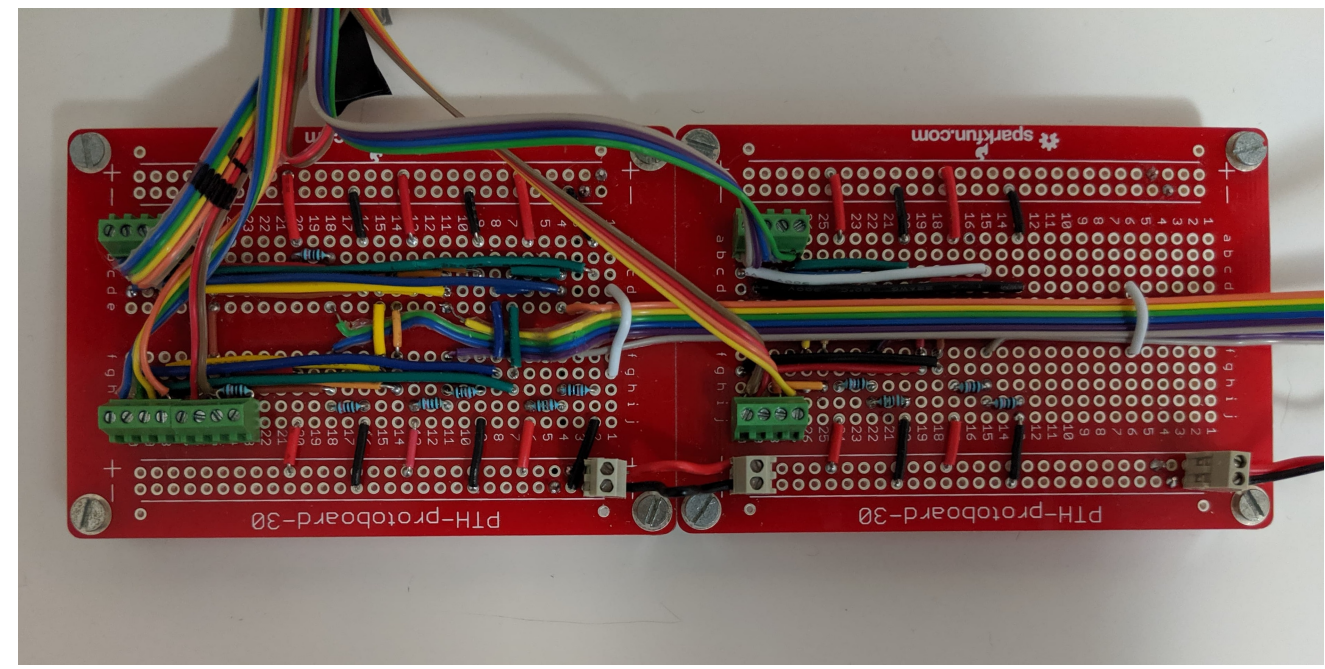

Figure 6.14: Assembled Perfboards

\subsubsection{Data Processing}

To analyze the data collected, the values from the strain gauges were further converted to digital signals. For this application, data processing was completed through the use of a LabJack T7-Pro DAQ. High-speed data acquisition was possible with the 16-bit analog inputs. The sampling rate of the device was dependent on the number of channels and the connection type to the host computer. In the streaming mode, for one channel, a maximum of 100 ksamples/ second was possible. Data collection was completed through a MATLAB code which was edited from the basic code structure provided by LabJack. This code can be viewed in Appendix F. The TH-Pro allowed bridge outputs to be read as a differential input. From the theoretical analysis, completed in Section 5.3.1, it was noted that final voltage outputs would require further amplification to ensure readable inputs. Amplification could be completed internally at the $T \%$. However, to minimize the amplification of wire noise, it was determined 
that amplification at a point closer to the bridge circuitry would provide a more stable output. Additionally, an expansion terminal board could be applied to minimize the distance from the DAQ to the prototype. Therefore, amplification with external circuitry was reviewed which could be connected to the terminal board.

External amplification could be achieved through the use of a LabJack InAmp. This instrumentation amplifier was noted as ideal for low-level signals such as those outputs from bridge circuits. The amplifier has 5 preset gain settings up to x201 amplification as well as an output voltage offset. A benefit of use of the InAmps was the output was converted from the bridge differential output to a single-ended output. This reduced the number of analog inputs to read at the T\%-Pro. An amplifier was acquired for each bridge. Theoretical analysis predicted use of the highest gain setting, however this was further tested experimentally.

Use of the Tr-Pro and InAmps were reviewed experimentally before integrating into the key prototype. Initial testing was completed with half-bridge circuitry for a cantilever beam, see Figure 6.15. The T\%-Pro was connected directly to an InAmp. The InAmp received the differential output of a half-bridge design, for a bending load application. Testing was completed with load applications to review the accuracy of the commercial instrumentation. Bridge voltages were compared between the direct DAQ connection and connection though the amplifier, at varying gain settings. The optimal data collection settings were verified through these tests. It was confirmed that use of the InAmps was effective in reducing additional noise due to wire resistance. The InAmps carried approximately 1 - $2 \mathrm{mV}$ of noise at the x201 amplification. This indicated that voltage outputs in this range may be subject to being "lost" within the system noise. It was further noted that any misalignment errors in the gauges were greatly amplified at higher gain settings. Therefore, in testing with 
the prototype it was noted that misalignment would need to be closely considered. Determination of the required gain was theoretically predicted. However, with the identification of the large influence misalignment error would have on the system, it was confirmed that the exact gain would be required to be found during initial testing.

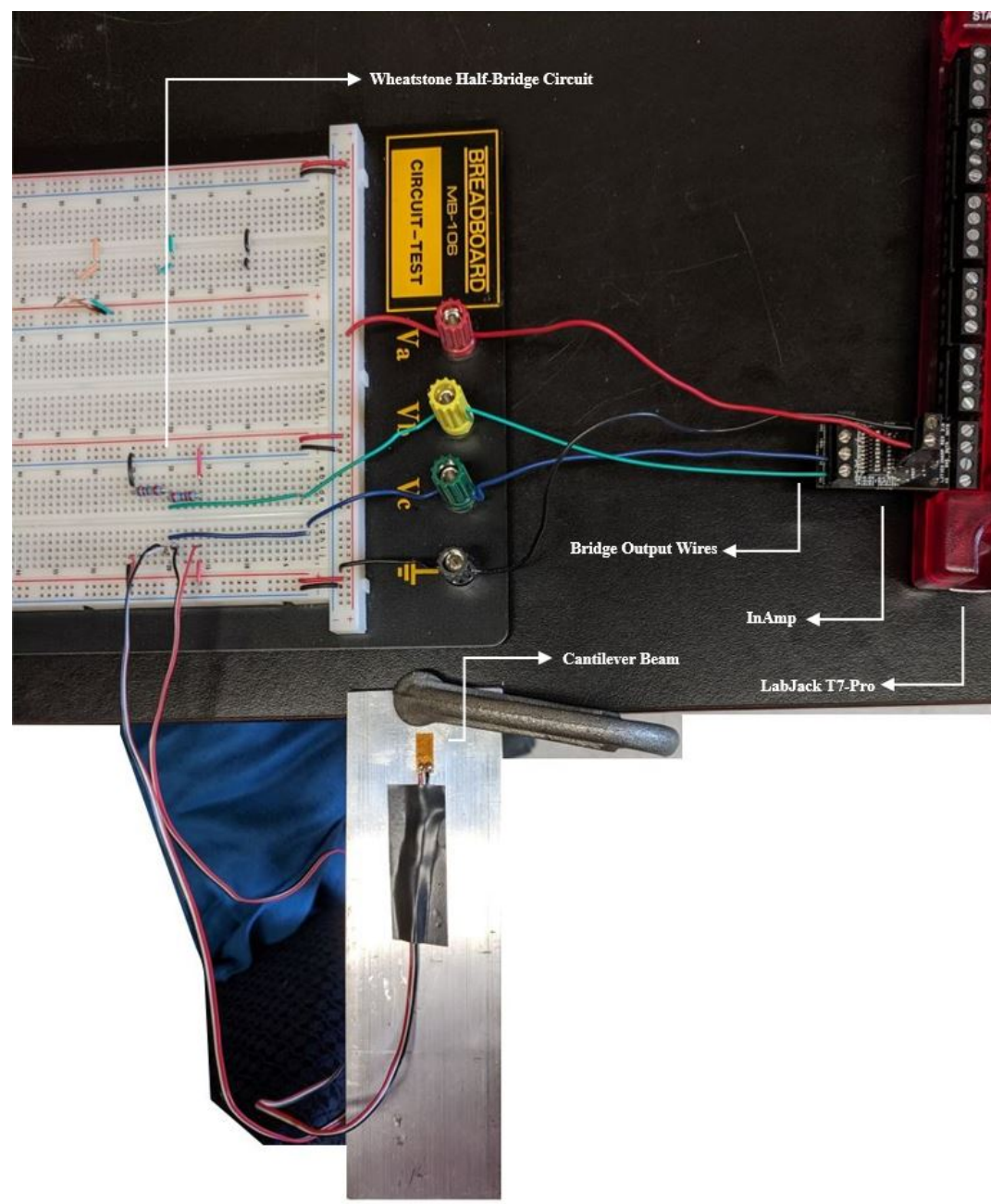

Figure 6.15: Cantilever Beam Test Configuration

In the initial testing stages of the prototype an identical excitation voltage was applied across all bridges. The standard excitation voltage of $3 \mathrm{~V}$ was applied. Although 
a higher voltage would result in a higher bridge output voltage reading, it would additionally carry an increased level of error, due to thermal effects generated by the current flowing in the gauges. Therefore, with consideration of the small outputs to detect, a lower excitation voltage was applied. The power supply ground was also connected to the T7-Pro through the SGND terminal. This was connected as the external power system could share a common ground with the T\%. This terminal has a self-resetting thermal fuse in series with the system GND.

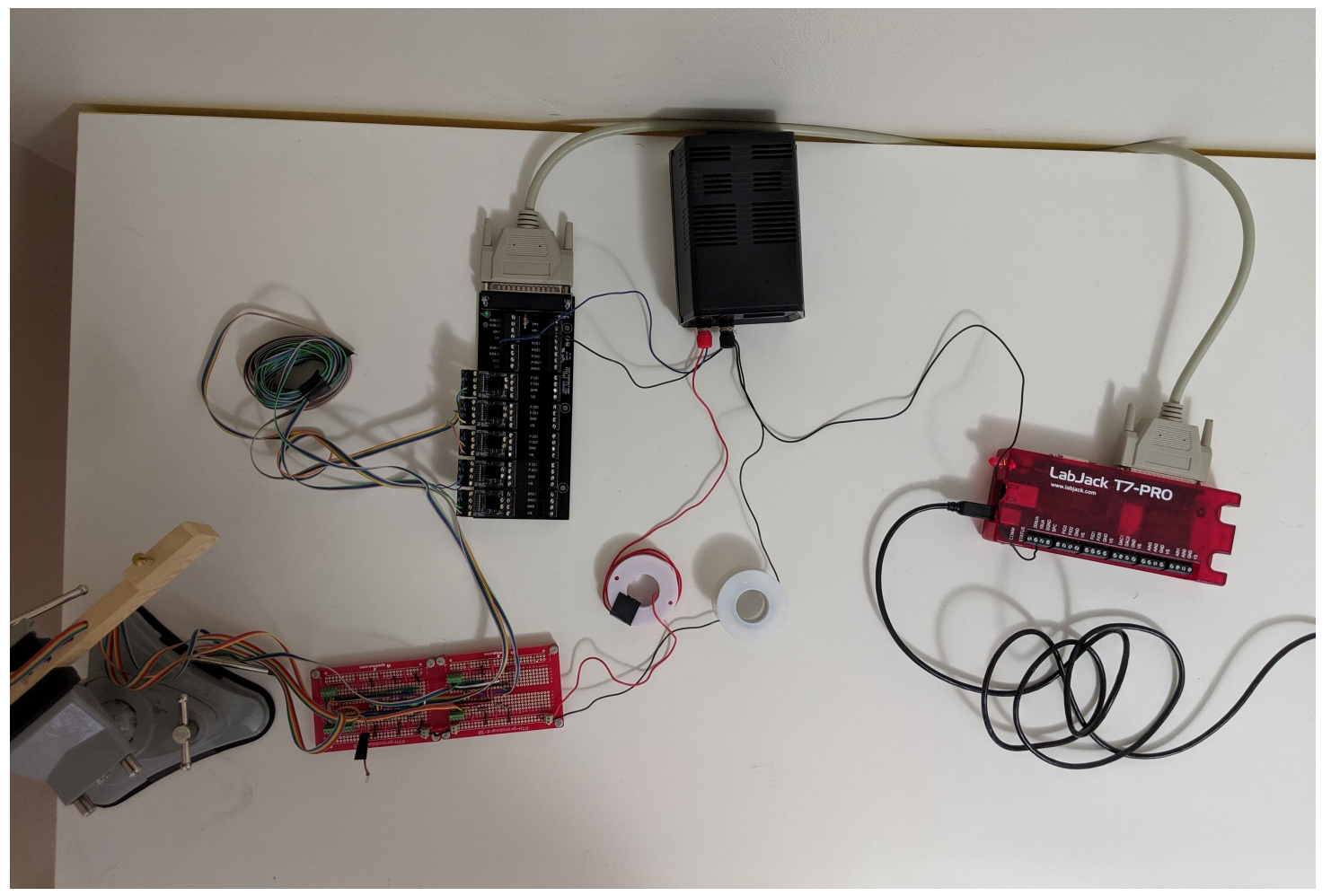

Figure 6.16: Benchtop Testing Setup for Key Prototype

Upon confirmation of the data acquisition system, initial testing was completed with the full prototype setup outside of the piano key, see Figure 6.16. Static data collection and simple loading cases were applied to compare the potential gain settings. It was confirmed that the x201 gain setting was most effective for each input bridge. The voltage offset of $0.4 \mathrm{~V}$ was applied to each InAmp. The applied offset, 
which was a manufacturer requirement with the use of InAmps, did result in some trade-offs in the data collection. An option originally assessed with the signal amplification was to amplify at the $\operatorname{InAmp}$ and then again at the $T \%$. This would allow a lower amplification of the signal noise, making the true signal more distinguishable However, due to the offset applied, internal amplification was not possible, as the output at the InAmp pushed the voltage to a range which would not fall within the internal amplification boundaries.

It was expected that at a zero loading condition, each bridge output would read $0.4 \mathrm{~V}$. However, due to misalignment, and asymmetry of bridge properties, this was not the case; each bridge varied in their unloaded voltage output. Theoretically, the voltage difference across each bridge when no load is applied is 0 . Due to the asymmetry across the bridges, this was not apparent experimentally. This was a factor of the differences in wire length, solder connections, and gauge inaccuracies. With the misalignment error identified, it was noted that final calibration could compensate for these offset errors. An example of the voltage readings is displayed in Figure 6.17 and Figure 6.18. This stage provided verification that all gauges were functioning as expected. It additionally confirmed that the system was sensitive to low force variations.

Completion of this process did identify additional errors, which required corrections, before validating the data outputs of the system. One such error was supply voltage instability. An external power supply was selected due to the number of bridge circuits in the design. A commercially available power supply was used to provide a prototype setup with equipment which was universally available for both future testing and design reproduction. However, it was noted that in initial testing that the power supply was drifting; it did not stay at a steady value when no load was 


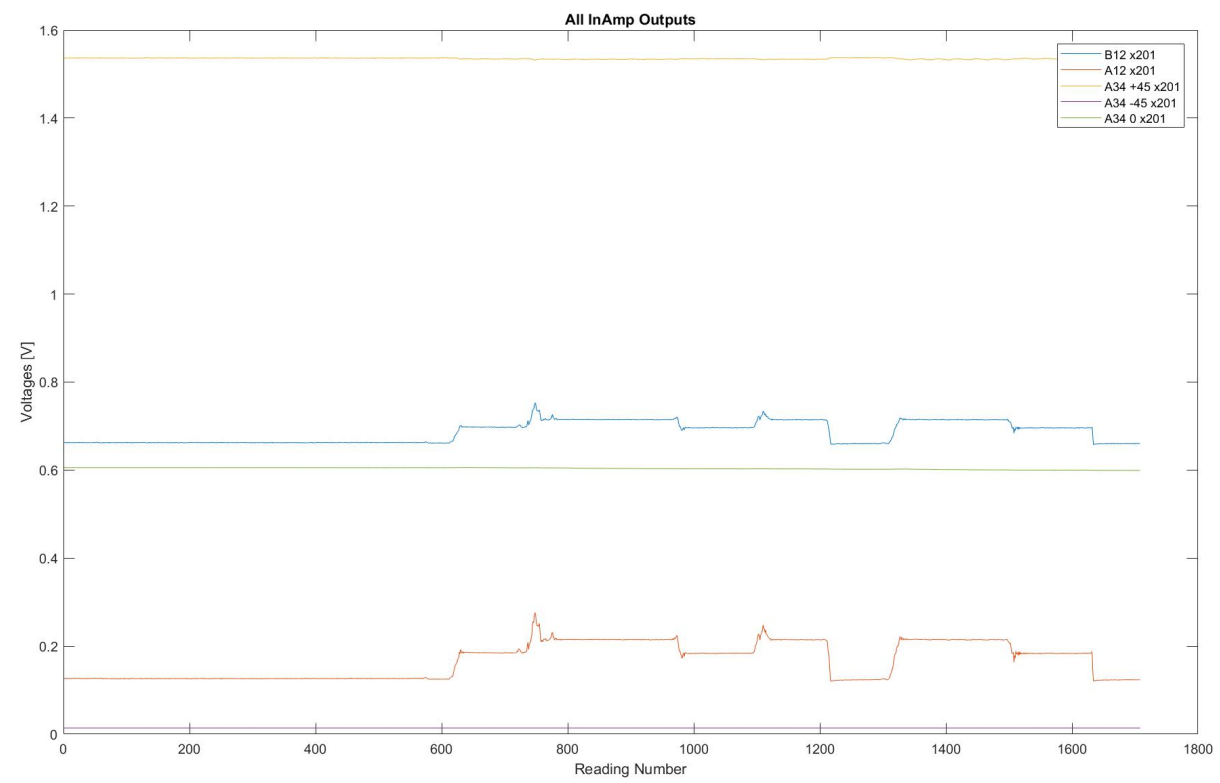

Figure 6.17: Fz Force Application between Unloaded, Mid-load, and High-load Applications Across Each Gauge Channel

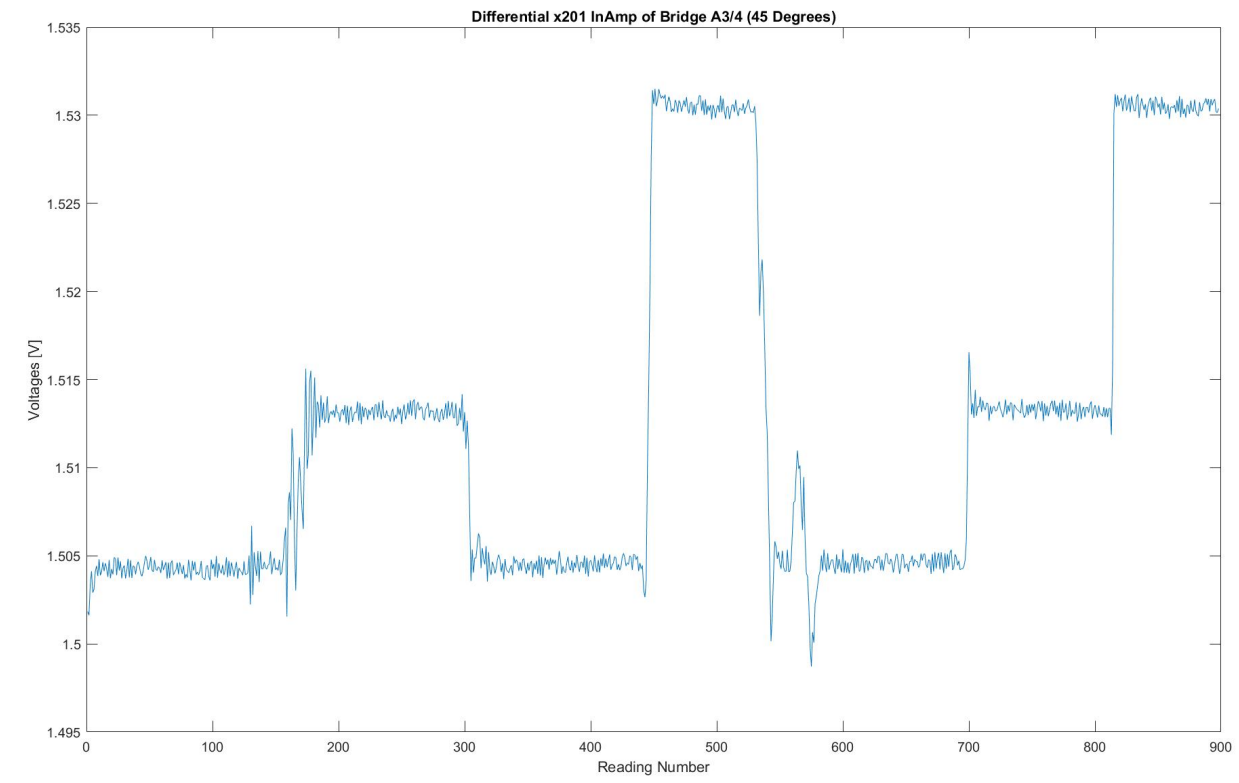

Figure 6.18: $F x$ Force Application between Unloaded, Mid-load, and High-load Applications Displaying the Voltage Outputs for One Half-Bridge 
applied. This was a concern, it is expected that the power remains steady to allow consistent readings under any conditions. To resolve this issue, the power supply was allowed to run for 30 minutes before testing commenced. This allowed any settling time necessary. Additionally, the power and ground readings from the external supply were measured by the $T 7$ during all testing to assess any inconsistencies in the supply. Unexpected voltage fluctuations from the bridge channel outputs was also noted due to loose wires. Wire movement was resolved by further securing the wires leaving the prototype key with zip ties. Additionally, terminal blocks were further secured to ensure wire movement was not resulting in any residual movement of the wire in the terminal which may have impacted the connection to the board. Completion of this step allowed further data analysis to commence. 


\section{Chapter 7}

\section{Data Validation}

Upon completion of the sensing beam assembly, validation of the beam output data was required. To confirm the design's ability to be used in future user testing, a review of the voltage outputs was required to confirm all gauges behaved as expected under different loading conditions. The following chapter provides an outline of the data validation process. Section 7.1 summarizes the experimental design setup used to ensure all expected force profiles were considered. The resultant voltage outputs of each gauge from testing are summarized in Section 7.2. This section further assesses the functionality of the design decisions with respect to the final data outputs.

Section 7.3 provides detail on the mathematical calibration process which would be applied to relate the voltage data acquired from the DAQ to the user input.

Section 7.4 evaluated the ability of the design to meet the original design inputs. Finally, Section 7.5 outlined the necessary steps for key integration into the piano for future user testing. 


\subsection{Experimental Testing}

\subsubsection{Test Design and Data Collection}

Section 6.3.3 provided an initial review of the gauge voltage responses during varied load application. The main objective of this stage of experimental testing was to extensively assess the response of the sensing beam under different loading and position cases. To effectively validate the design, the range of expected keystroke inputs would need to be simulated. Therefore, a series of tests were required. Overall, the steps of this process would include: planning the experimental methods, completing the measurements, plotting the results, and then verifying their effectiveness in responding to the loading conditions.

The first step in testing was to determine the method of completing the experimental trials. As outlined, the experiment design would require variance in loading position as well as the force applied. Various methods could be used to approach this measurement goal. Each method would need to be considered on the basis of the experimental setup, machining requirements, and the accuracy and repeatability of the trials.

One possible method would be to utilize a MTS Compression machine. The MTS could be used to provide accurate force applications onto the prototype key. This method would carry a high level of accuracy and repeatability, but it would require additional parts to be machined to complete the testing rig. To vary the location and direction of force application, a custom base to secure the prototype key in the MTS would be required. Additionally, to limit the area of force application on the key, a custom head for the MTS would also need to be machined. Overall, this method would provide accuracy in the data inputs, but would require extensive machining to 
create a custom base and head for the MTS. Similar to this method, but on a smaller scale, would be to develop a setup with a force actuator. A track could be assembled to support the actuator, and vary its $\mathrm{x}$ - and $\mathrm{y}$ - position. This would provide a setup to test the necessary loading cases. Again, this method would require machining, as well as possible verification and calibration of the motor force actuator.

Another method of assessment would be to apply measured masses to reach the desired loading cases. This could be achieved through one of two methods. The first option would be to develop a mass hanger in which measured masses could be hung. Set positions could be marked, or indented on a keytop to provide accuracy and repeatability in the measurements. The second option would be to hang the masses on a wire, which could be varied in position. This would provide a simple setup which could be adjusted to allow testing in the different directions of force application. Both of these methods would provide a simplified test setup, and when executed correctly, provide accurate results. These methods would require a settling time after load application to account for any sway from the load application.

Overall, for the simplicity and effectiveness of the testing, the wire and mass method was selected. Before moving forward with the experimental design, a final decision on the scope of the system was necessary. The sensing beam could be assessed externally from the key, or with the key front attached. To examine the key as a fully-ready system for testing it was determined that the system would be tested as a whole. Therefore, the key was fully assembled, including the gauge wiring being secured in their final position. Addition of the key front would provide the ability to vary the $\mathrm{x}$ - and $\mathrm{y}$ - position of the wire to test combined loading cases.

After confirmation of the experimental method, the range of measurement trials to be applied was determined. To effectively validate the accuracy and precision 
of the current design, voltage collection trials would need to be completed over a variety of testing conditions, and repeated across each trial. To assess the multiaxis measurement, direction of load application would need to be varied. To further consider the possible applied moments on the beam, the location of load application would need to additionally be varied. With the addition of the key front, it was determined that a spare keytop could be marked and used solely for calibration. Using the guide pin hole in the key front, the fishing line wire was threaded through the body of the key. A small hole was made in the keytop support, and a 1/64 inch bit was used to drill a centred hole in a plastic keytop. This was completed to ensure the fishing line threaded through the hole would have limited movement. With the wire centred in the key, additional positions of measurement were made. To allow measurement variation in the $\mathrm{x}$ - and $\mathrm{y}$-position a total of 8 testing positions were made, shown in Figure 7.1 and Figure 7.2.

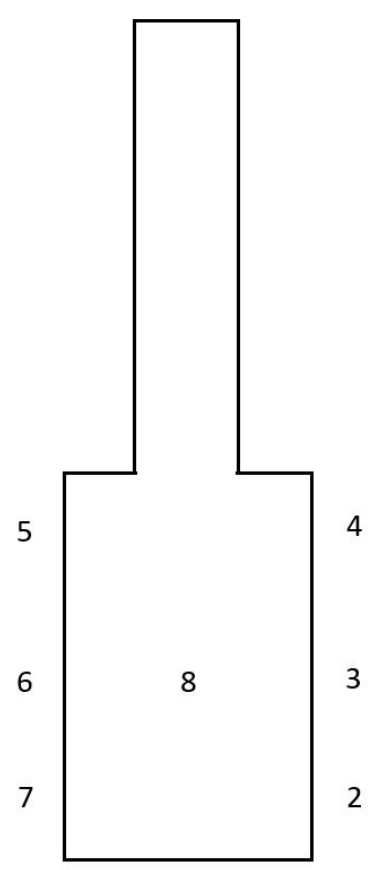

1

Figure 7.1: Defined Positions of Measurement on the Experimental Keytop 


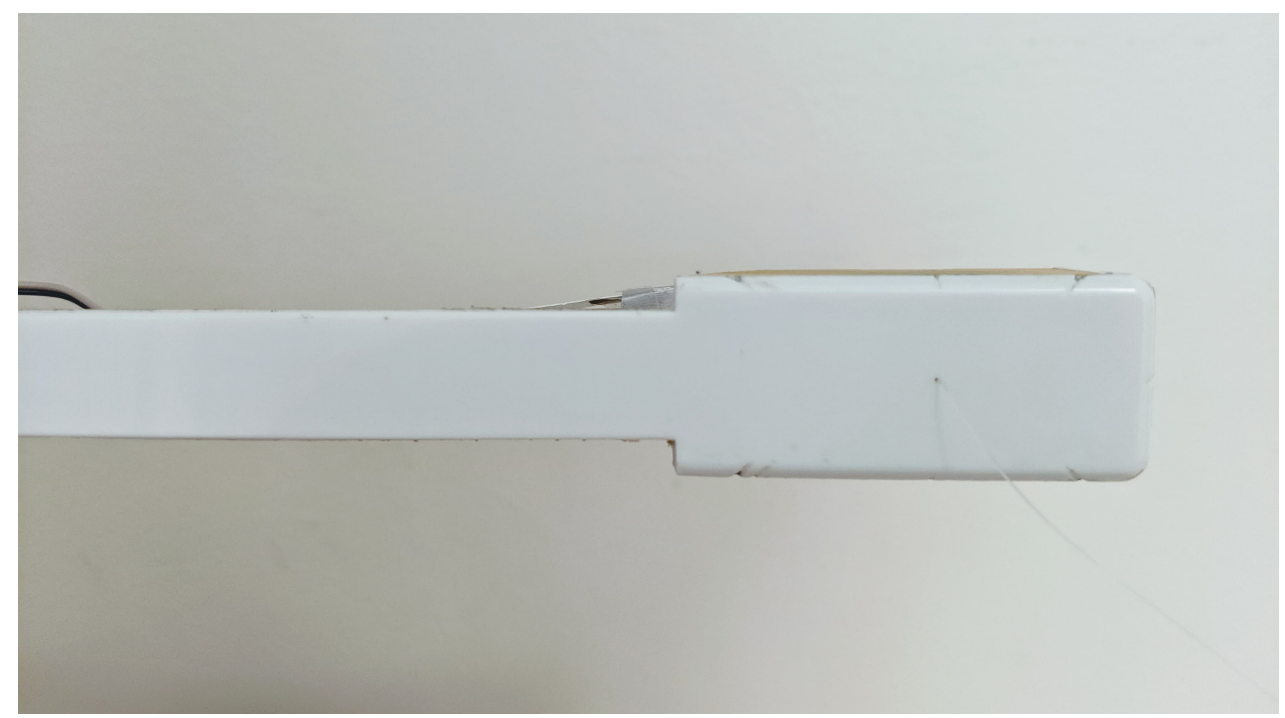

Figure 7.2: Experimental Keytop

To measure various directions of loading, the angle of the key would need to be altered. The use of a 360 Adjustable Table Vice was applied to allow different angles of measurement, and a digital level was used to ensure correct alignment of the key within the vice. The measurement positions, defined above, would not provide a true analysis of the response of the gauges for all loading directions. Therefore, the number of position and angles tested were determined for each direction of load application. First, in the isolation of $F_{z}$, one angle of the key was used, that being the standard position of the key in the piano. In this angle, the 8 pre-defined testing positions would be applied to assess the variance in $\mathrm{x}-$ and $-\mathrm{y}$ position. In the isolation of $F_{x}$, two angles would be applied, being that of rotation of the key $90^{\circ}$ in either direction along the x-axis. This would allow evaluation of the gauge responses on either side of the bridge pair at $A 34$. In these orientations only one position, position 8 , would be required. Finally, in isolation of $F_{y}$, it was critical to assess both tension and compression loading. Therefore a total of 6 angles were assessed, ranging from the rotation of the key $-90^{\circ}$ to $+90^{\circ}$ along the y-axis. This final set of tests allowed a comparison of single-axis and multi-axis force application, as $F_{z}$ would comprise 
of a portion of the force profile. The variation of these angles of measurement can be viewed in Figure 7.3. Further details on the measurement orientations and load application positions can be viewed in Appendix G.

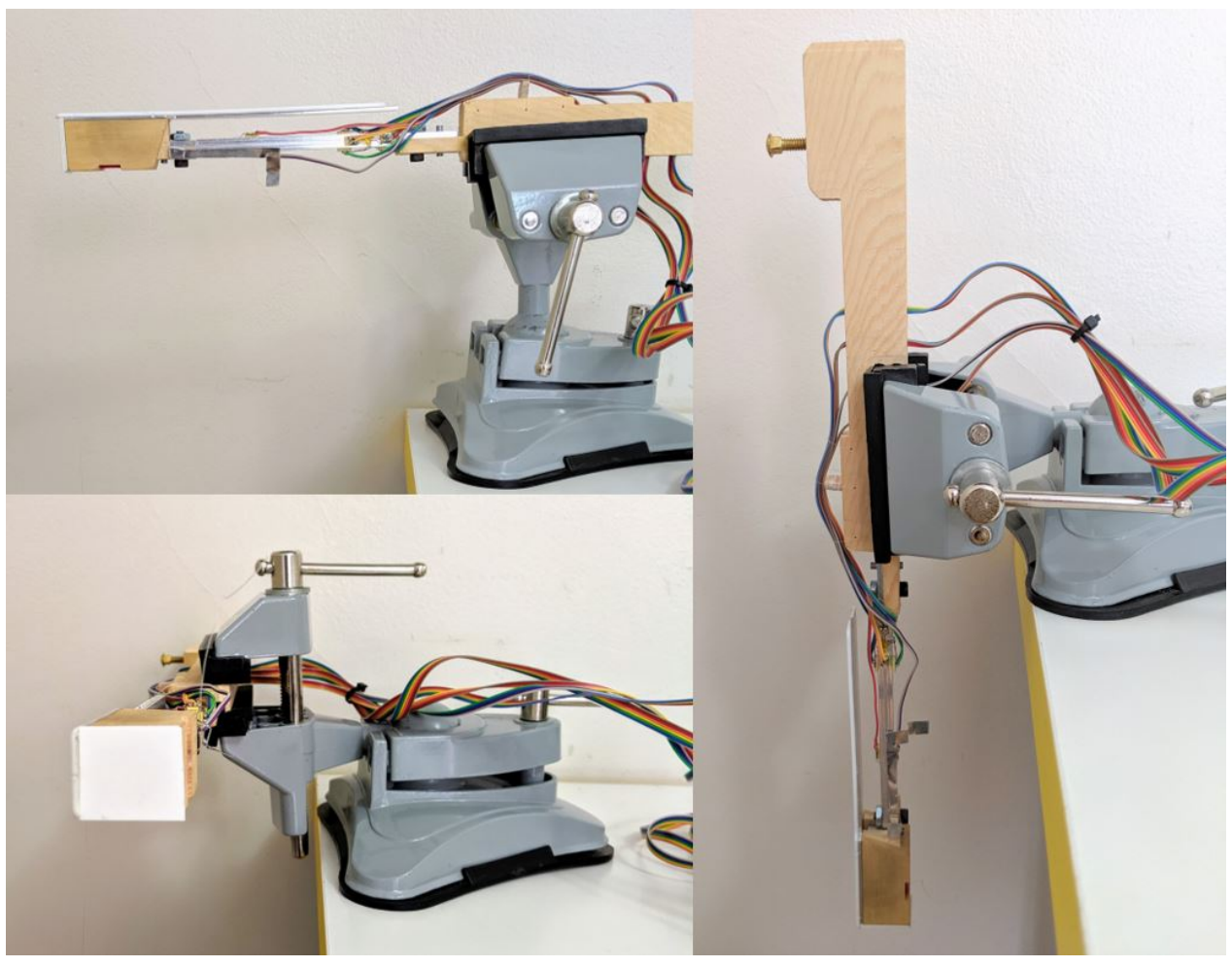

Figure 7.3: Angles of Measurement Used During Testing

The next determination was that of the number, and range, of loads to apply during a trial. Ideally, the range of loads applied should provide an increment small enough to evaluate the resolution of the device, as small-scale weight discrimination was desired. Additionally, the span of masses applied should assess a range of expected measurements to validate that the sensor retains accuracy throughout the expected measurement range. In this study, it was not practical to assess the sensing beam in a static position against the full range of loads, which were upwards of $60 \mathrm{~N}$ (which would require a $6.11 \mathrm{~kg}$ mass to be applied). The applied load of $60 \mathrm{~N}$ was selected to ensure the device could withstand impact. However, this level of load is not typical in 
most applications. Rather, the test load range was concentrated towards the centre of expected force input. This method has been applied in other areas of scientific calibration, see [72], and therefore was deemed acceptable for this first stage of data analysis testing. Piano technician regulation masses and coins were used to obtain the correct mass additions. As different maximum expected loads were previously determined for the $F_{z}$ loading case compared to $F_{x}$ and $F_{y}$, two tests ranges were applied. The list of the total range of the test cases applied, in terms of the masses added to the fishing wire, can be viewed in Table G.4. It should be noted that this is the nominal mass applied, and the not exact mass. In completing the data analysis, the purchased and designed masses were measured with a precision scale to account for any deviations from the nominal values provided. The precise measured masses, which were applied in the data analysis, and can be found in Appendix G.

For validation of the precision of the sensing beam outputs, redundant measurements would be required. Consideration of repeated testing was critical to evaluate the spread in measurements across each test case. If the prototype did not hold a high precision, it would not be beneficial to move on to calibration and user testing. This is because the response of the gauge may differ across different applications of the same load, meaning the true variable inputs would not be accurately calculated. Therefore this step was critical to the data validation process. To complete repeated testing, it was determined that the pre-defined test conditions would be repeated a minimum of 3 times across different days. This would expand the assessment of the potential data spread, to provide a true reading of the data outputs.

Experimental data collection was then completed for each of the defined loading cases. Time was allowed to ensure that any wire sway was negligible in the reading. An example of the collection process can be seen in Figure 7.4. The original data 
Table 7.1: Range of Test Cases Applied During Validation Data Collection

\begin{tabular}{|c|c|c|}
\hline & \multicolumn{2}{|c|}{ Mass Applied (g) } \\
\hline Case Number & $F_{z}$ Testing & $F_{x}$ and $F_{y}$ Testing \\
\hline 1 & 0 & 0 \\
\hline 2 & 32 & 32 \\
\hline 3 & 50 & 50 \\
\hline 4 & 51 & 51 \\
\hline 5 & 52 & 52 \\
\hline 6 & 53 & 53 \\
\hline 7 & 54 & 54 \\
\hline 8 & 55 & 55 \\
\hline 9 & 56 & 56 \\
\hline 10 & 57 & 57 \\
\hline 11 & 58 & 58 \\
\hline 12 & 59 & 59 \\
\hline 13 & 60 & 60 \\
\hline 14 & 100 & 100 \\
\hline 15 & 200 & 150 \\
\hline 16 & 300 & 200 \\
\hline 17 & 400 & 250 \\
\hline 18 & 500 & - \\
\hline 19 & 600 & - \\
\hline 20 & 700 & - \\
\hline 21 & 800 & - \\
\hline 22 & 900 & - \\
\hline 23 & 1000 & - \\
\hline
\end{tabular}




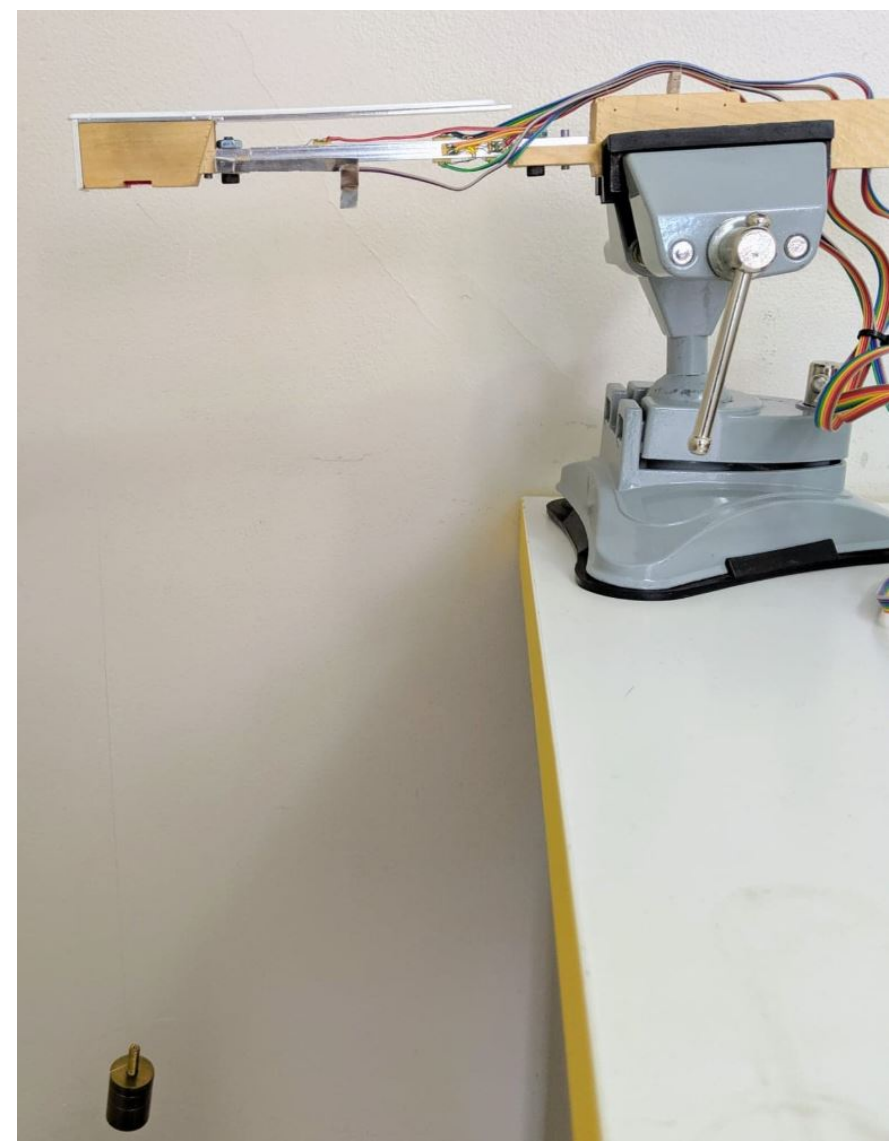

Figure 7.4: Application of Measured Masses for Data Collection

collection $M A T L A B$ script was edited to collect the known input values as well as the average voltage outputs across each gauge. To do so, 2000 scans were completed over a 2-second interval, in which the mass applied remained static. The mean across all data collected was then calculated to provide an average voltage reading. The standard deviation was additionally calculated. Finally, the power supply was also recorded. This script, provided in Appendix H, was used to store the data from each testing case into a .csv file. In total, 1144 testing cases were completed (as the $F_{z}$ testing was repeated 4 times to evaluate an outlier). Therefore, across the 5 gauges, this provided 5720 voltage readings to assess. 


\section{$7.2 \quad$ Findings}

After completion of the data analysis, the results of the voltage recordings were

plotted. The full set of data can be viewed in Appendix G. Review of the raw voltage data allowed confirmation that the bridges were responding the the applied loads as expected. It was previously determined in Chapter 6 that each voltage channel carried a different offset due to amplification of residual error in the system. Therefore, to assess the bridge responses together, the data were first passed through to remove the initial offset in each channel. This provided visual data sets that focussed on the rate of change of the voltage responses under the varied loading cases, rather than considering their offset from each other.

The voltage outputs were first reviewed to confirm the precision of each bridge pair. To do so, the results of the repeated trials, in which the position of load application remained the same, were reviewed. If the voltage responses were not reproducible over different trials, which spanned various days of testing, the key would be unable to be considered for future calibration and user testing. This process was first completed with the gauge responses to the trials completed for each position of $F_{z}$.

The response over the repeated trials was graphed for each bridge output for every position of $F_{z}$ testing. An example for this, at Position 2, can be viewed in Figures 7.5 and 7.6. The responses from the linear gauge bridge outputs, Figure 7.5, displayed a high precision in the data outputs between the repeated trials. A similar trend was noted across the other measurement positions as well. This stage of testing validated the gauges were able to be used over different testing periods, and testing conditions, to obtain the same results. It was apparent that the gauges offered a high repeatability as there was a high precision between the voltage outputs across each trial. 


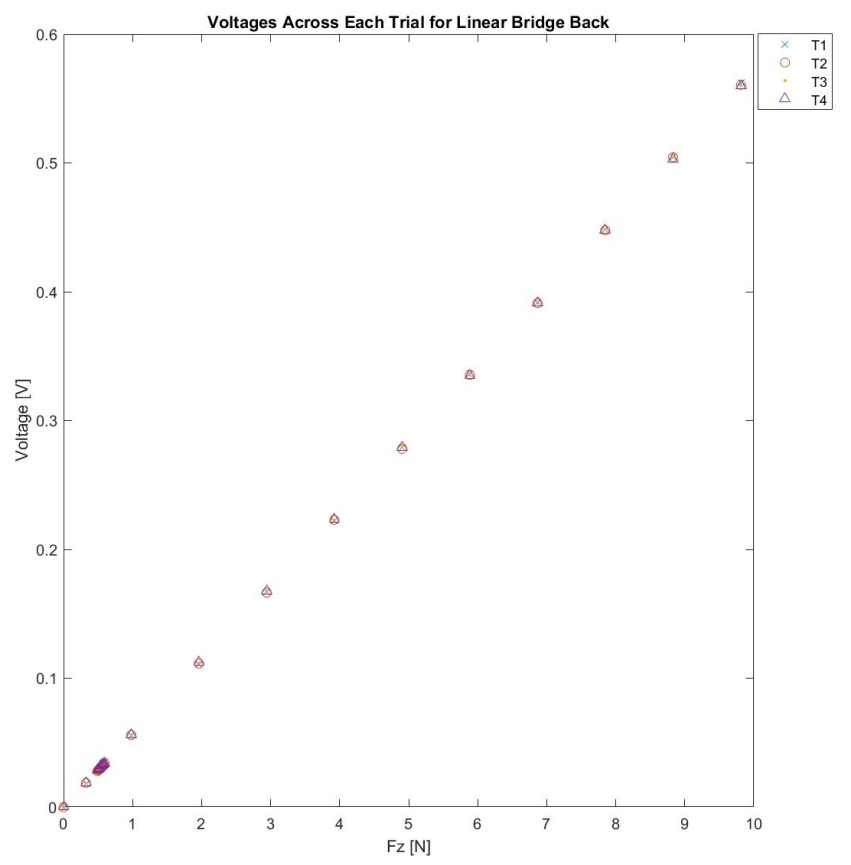

(a) Plane A Response

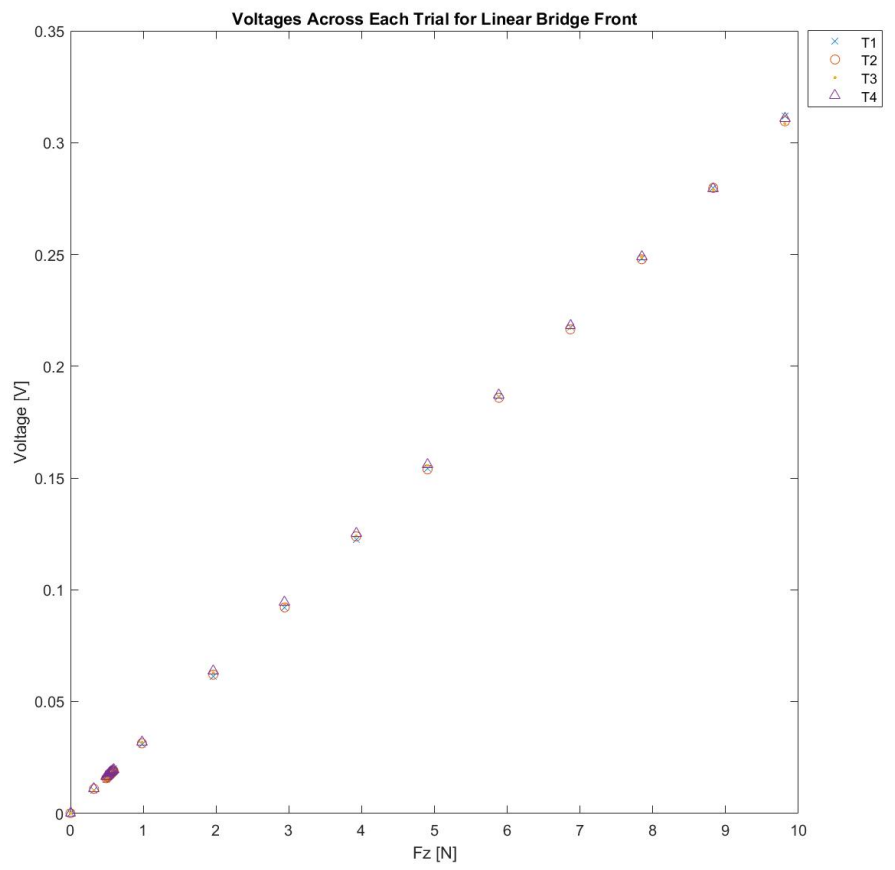

(b) Plane B Response

Figure 7.5: Voltage Responses of Repeated Trials at Position 2 for the Linear Gauge Pairs 


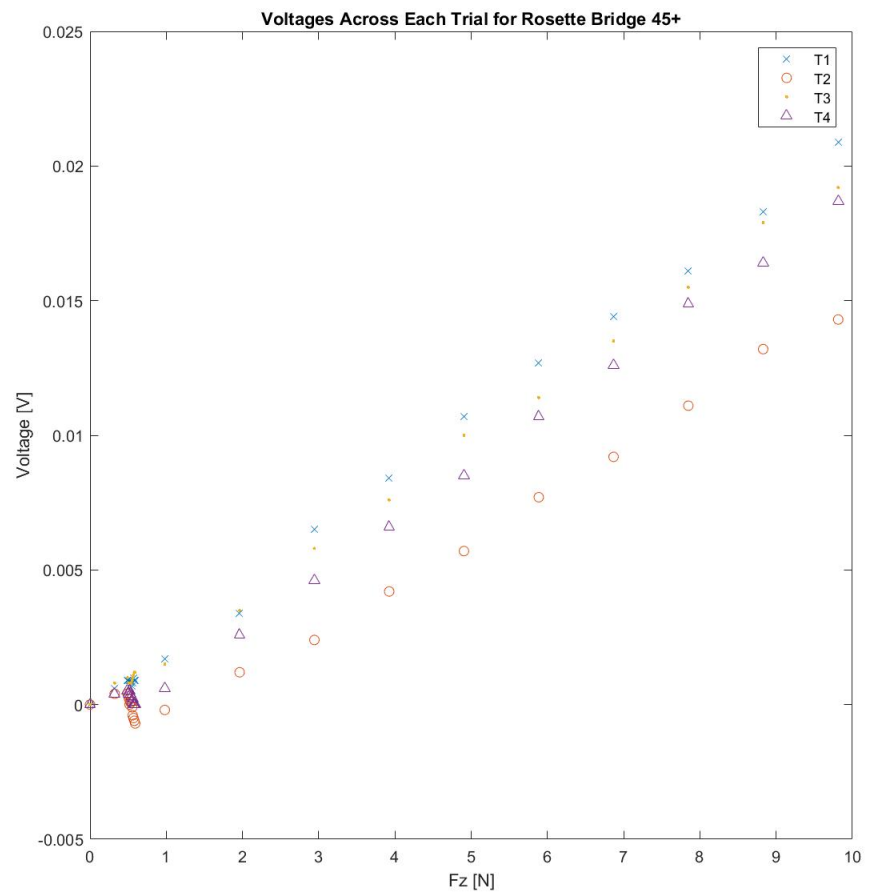

(a) Rosette $45^{\circ}(+)$ Response

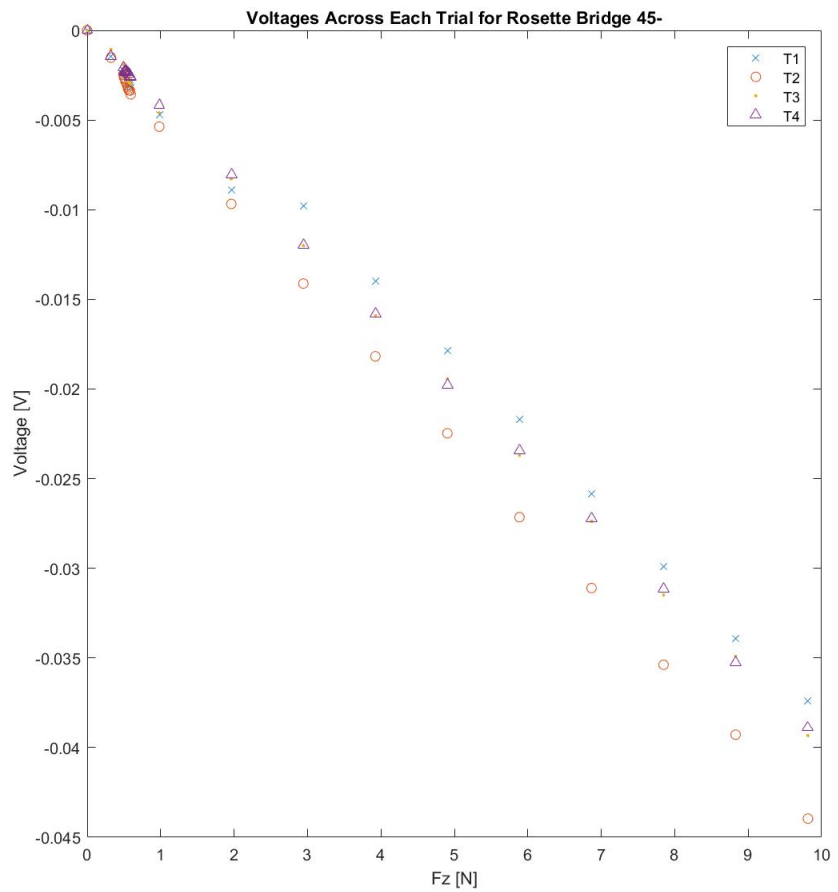

(b) Rosette $45^{\circ}$ (-) Response 


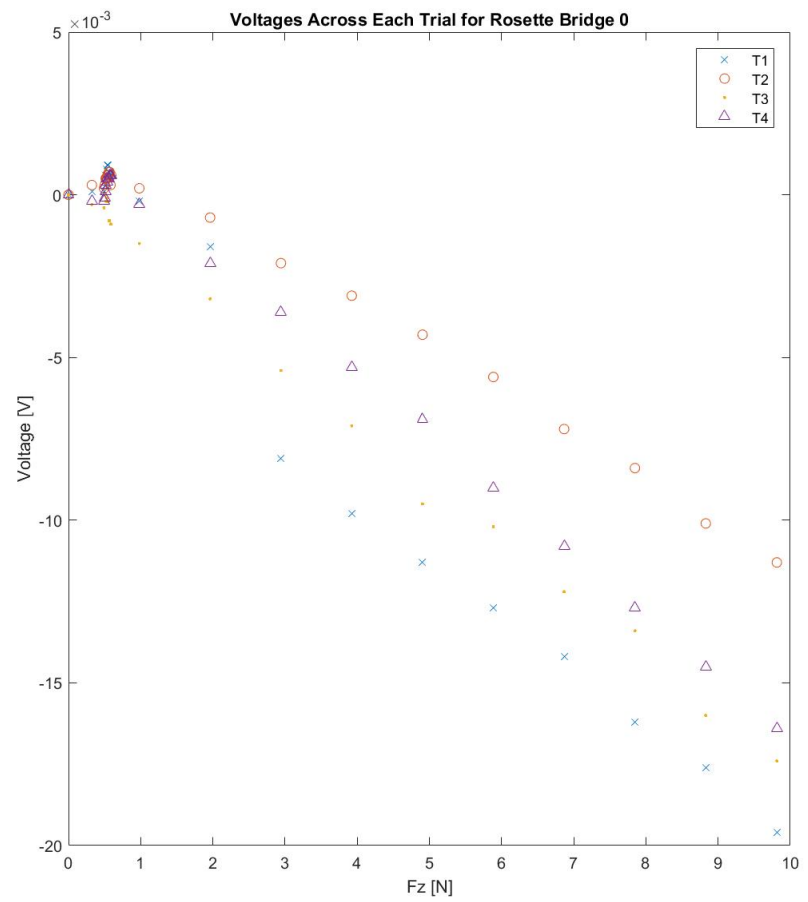

(c) Rosette $0^{\circ}$ Response

Figure 7.6: Voltage Responses of Repeated Trials at Position 2 for the Rosette Gauge Pairs 
In examining the precision of the rosette bridge outputs, they were noted to have a much lower precision. A $5 \mathrm{mV}$ spread was calculated across the upper range of the repeated trials. This spread was significant in this testing application. This low precision would result in data processing errors, and a loss of resolution from the rosette gauges. As the full voltage span from these gauges was expected to remain low, due to the force applications they were detecting, this was an indicator that the gauges may not be sufficient to measure the necessary loads. Position 2 was selected for this review, as it contained an x-position offset, which the rosettes were sensitive to. However, it was not expected that the rosette gauges would have a very significant response. Before drawing any conclusions on the use of the gauges, it was important to further assess the $F_{x}$ and $F_{y}$ loading cases.

Before moving on to other loading conditions, the $F_{z}$ cases were further reviewed. First, the voltage outputs were examined at each trial to see the overall trend of the data. An example of this is again displayed for Position 2, see Figure 7.7. This allowed a review of the magnitude of the bridge responses under an increase in load application. The channel responses behaved as originally predicted from the theoretical analysis. These results were as expected, as the linear gauges were more sensitive to the $F_{z}$ loading. The gauges of the strain rosette were less sensitive, and had a lower span in their overall response. The linear bridge pairs showed a strong positive response to increased loading cases. The magnitude of the responses was much stronger than those of the rosettes. In comparing the gauges placed at the back of the beam, Plane $A$, and the front of the beam, Plane $B$, they also behaved as expected. Due to the increase in moment arm, it has been noted that the gauges at Plane $A$ would have a higher voltage response. This was validated in this testing stage. It was noted that the slope of the gauge pair at Plane $A$ was significantly steeper. The range of the voltages at these mass applications was approximately $0.56 \mathrm{~V}$ and 
$0.31 \mathrm{~V}$, for the Plane $A$ and Plane $B$ positions, respectively. This further validated that the two planes could be used to determine the y-position of touch, as the voltage outputs did display a different magnitude in response to the applied loads. As the $\mathrm{y}$-position along the beam was varied, the voltage responses too were changed. A smaller moment arm, resulted in a lower voltage output. However, the relationship between the two planes remained the same.

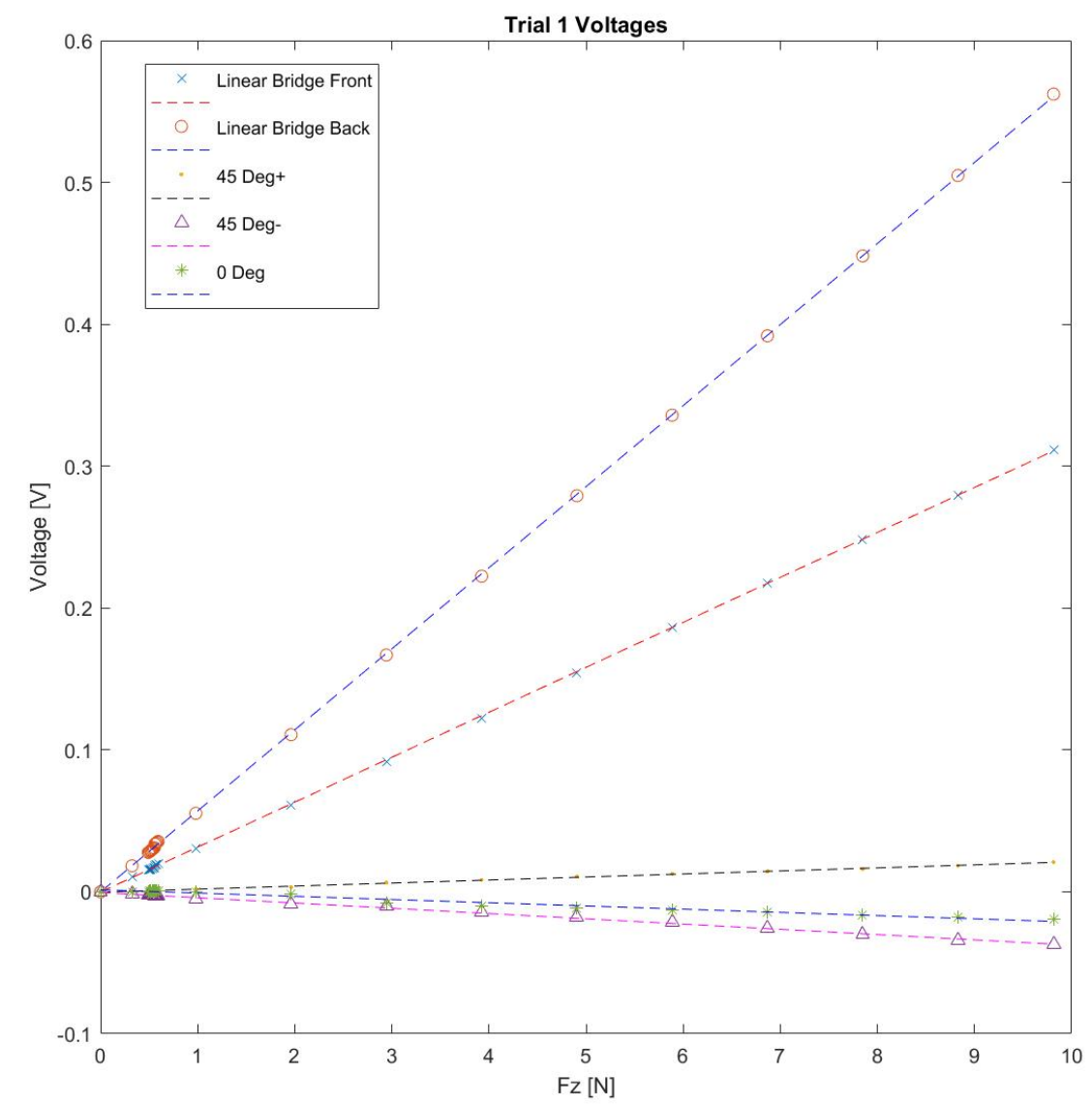

Figure 7.7: Voltage Responses of All Bridge Outputs at Position 2, Trial 1

Additional responses from the 8 positions of loading used in the $F_{z}$ loading cases can be viewed in Appendix G. The process of assessing the precision and bridge responses 
was repeated across all trials and $F_{z}$ loading positions. This was necessary to evaluate if the response of the gauges would vary at different positions of measurement, or under different loading conditions. From the review of all $F_{z}$ loading cases a few deviations from the expected outcomes were noted. Across the rosette responses, the results a trial 2 appeared to be offset from the other trials. The power supply voltages were reviewed to ensure the power supply was stable throughout the trials, see Figure 7.8.

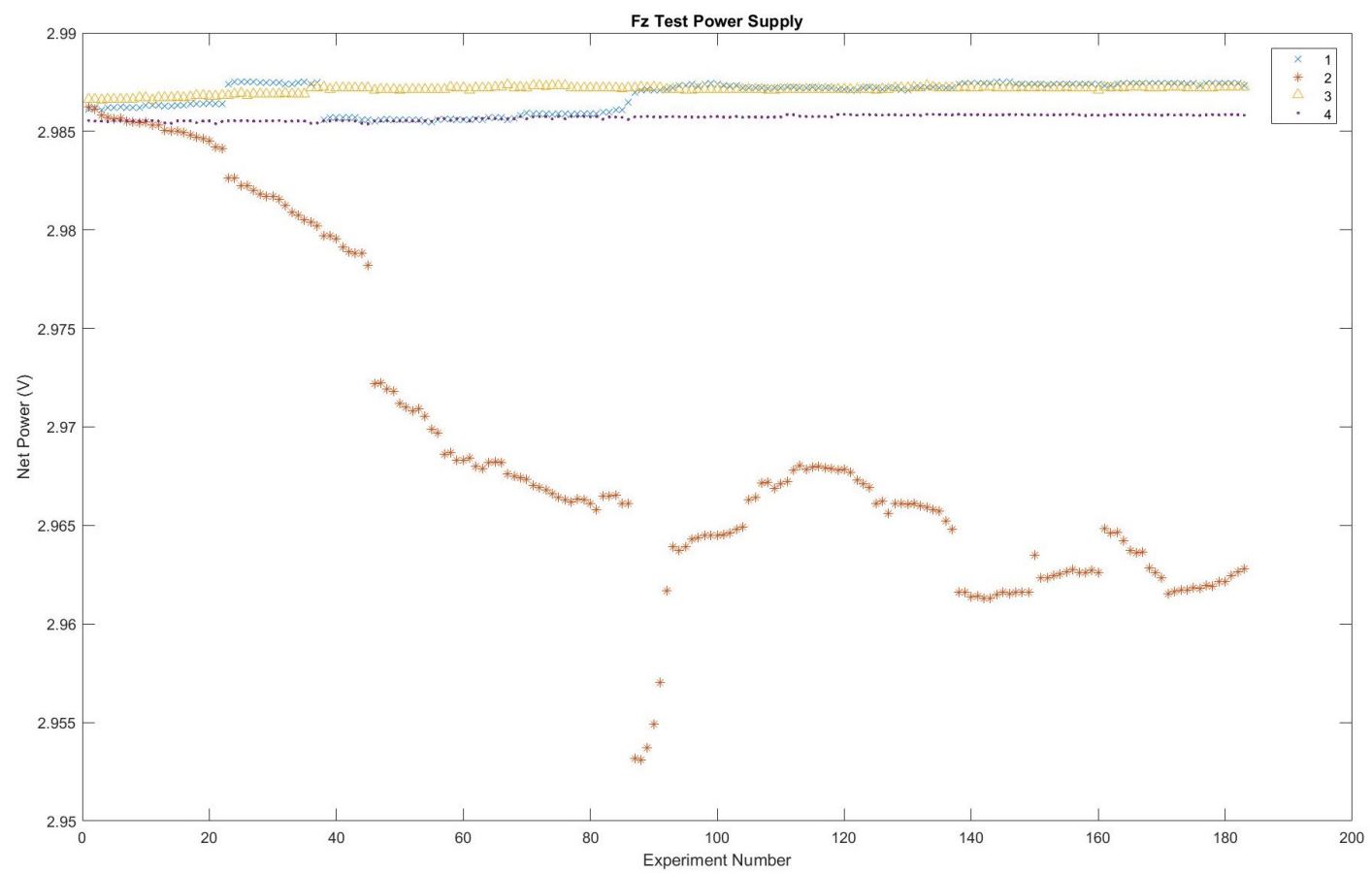

Figure 7.8: Power Supply Reading at Each Loading Case Across Each Trial for $F_{z}$ Load Applications

Trials 1,3 , and 4 noted a range in the power supply voltage of under $2 \mathrm{mV}$. This range was previously measured in Chapter 6, and was therefore expected. However, the power supply from trial 2 was not stable. A range upwards of $30 \mathrm{mV}$ was noted across trial 2. This would have significantly impacted the bridge output responses. 
This supply voltage appeared to be an outlier among the other trials completed. This further indicated the importance of collecting the power supply voltage to ensure supply fluctuations were not present. It would also provide the potential to use the fluctuations as a feedback in the system to keep a steady bridge response across all channels.

Another deviation from the expected voltages was a dip in the rosette values at the $1 \mathrm{~g}$ mass increments. A small step shift in the voltage response was noted across each rosette gauge at the $0.5 \mathrm{~N}$ test increment. This occurred across multiple trials. It appeared that the voltage readings shifted down and continued reading at this shifted position. This was not found in the linear gauge responses, which were collected at the same time. Therefore, it was not expected to be an experimental error. Gauge installation, including installation procedures and adhesive used, was completed following manufacturer's recommendations. These findings may suggest hysteresis in loading. It should be noted that the mass addition process may have had an impact on the gauges. Each time the load was incremented the total mass was essentially removed from the wire. This was completed to apply the additional masses. After this brief unloading, the new masses were then reapplied. The possible hysteresis response may have been attributed to this loading procedure.

The above process was then repeated with the $F_{x}$ loading cases. An example can be viewed in Figure 7.9. The rosettes responded with the expected high sensitivity to this loading condition, while the linear gauges were less sensitive. It should be noted that the magnitude of the responses were still significantly lower compared to the linear gauge responses to $F_{z}$ loading. When assessing the precision of each gauge over repeated trials, a similar trend as the $F_{z}$ loading cases was noted. Inconsistencies in the gauge precision was noted for the rosettes, at a similar scale to findings of the 
previous loading conditions.

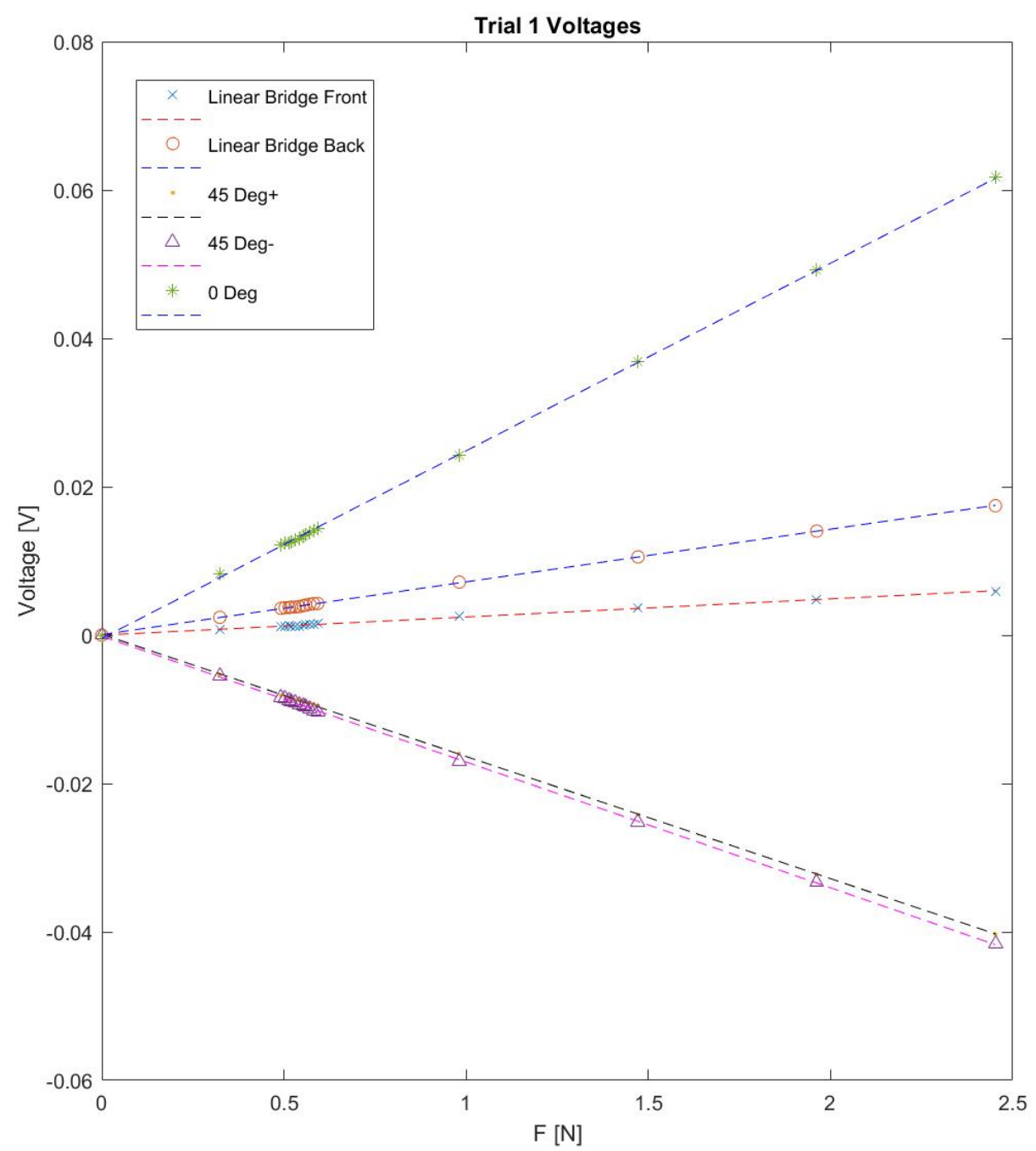

Figure 7.9: Voltage Response of All Bridge Ouputs at $-90^{\circ}$ Position, Trial 1 for the $F_{x}$ Load Application 
Finally, the results of the $F_{y}$ loading cases were reviewed. Trials were completed to evaluate tensile and compressive $F_{y}$ loads. As the angle varied, the total force component also consisted of $F_{z}$ loads. Figure 7.10 displays the rosette response in the pure tensile $F_{y}$ loading case. These tests further exemplified the inaccuracies of the rosettes. The general trend in the gauges throughout the load applications did behave as expected. However, and especially in the $1 \mathrm{~g}$ increment trials, the data lacked precision. Not only was the span of the data significant, but the magnitude of change among each loading cases differed across each trial. The error spanning the trials would eliminate the ability to distinguish the force input values. The rosettes would not be functional in detecting low force inputs in this application.

\subsubsection{Conclusions}

The linear gauge pairs showed a high accuracy and precision across all loading conditions. The gauges behaved as expected to each load applied. Additionally, the magnitude of the voltage response was appropriate for this application. The span of the voltage range was large enough to distinguish the voltage response against any noise present in the system. A smooth data response was noted from the continuous data collection in Chapter 6 as well as the point data collection tests completed in the experimental load cases. These results provided confidence in the selection and use of the linear strain gauges.

It was apparent that errors existed in the rosette responses. The gauges did not respond with the necessary precision to validate their use to detect the required loading conditions. The discrimination in the values was most apparent at the $1 \mathrm{~g}$ variation level of testing. Mounting error may have resulted in residual error in the gauge pair which may have impacted the overall resolution of the bridge responses. Additionally, the errors could be attributed to the magnitude of the detectable forces 


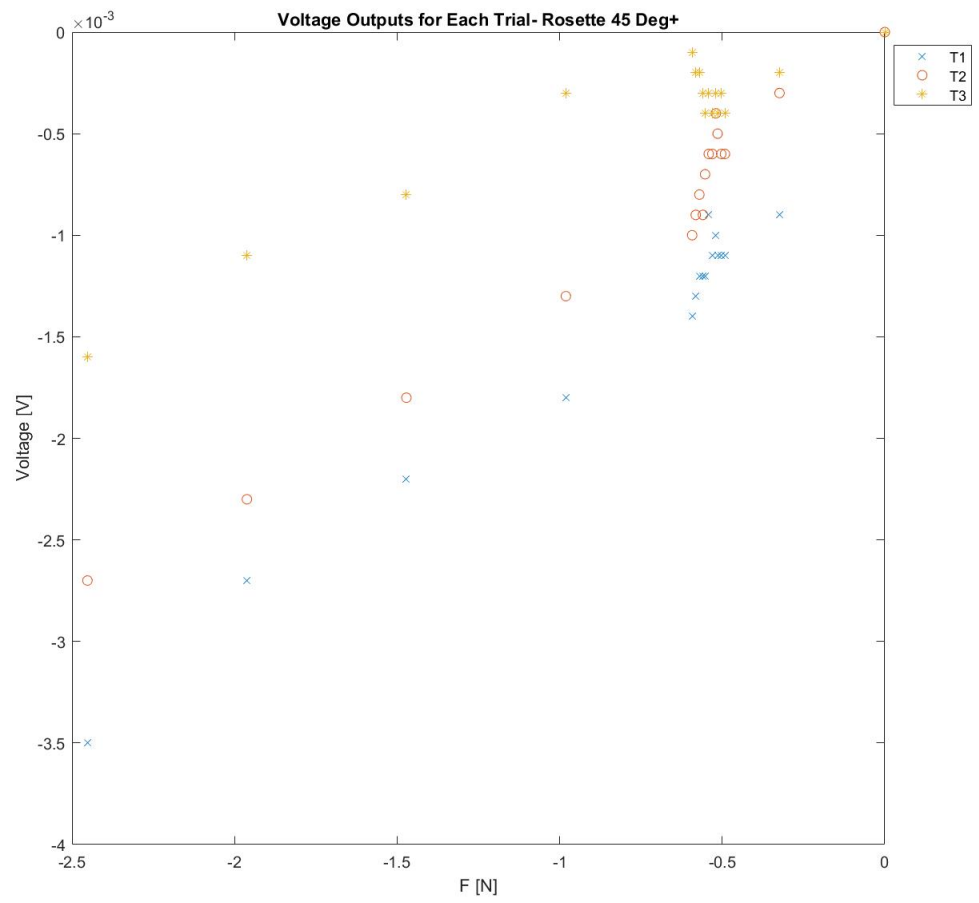

(a) Rosette $45^{\circ}(+)$ Response

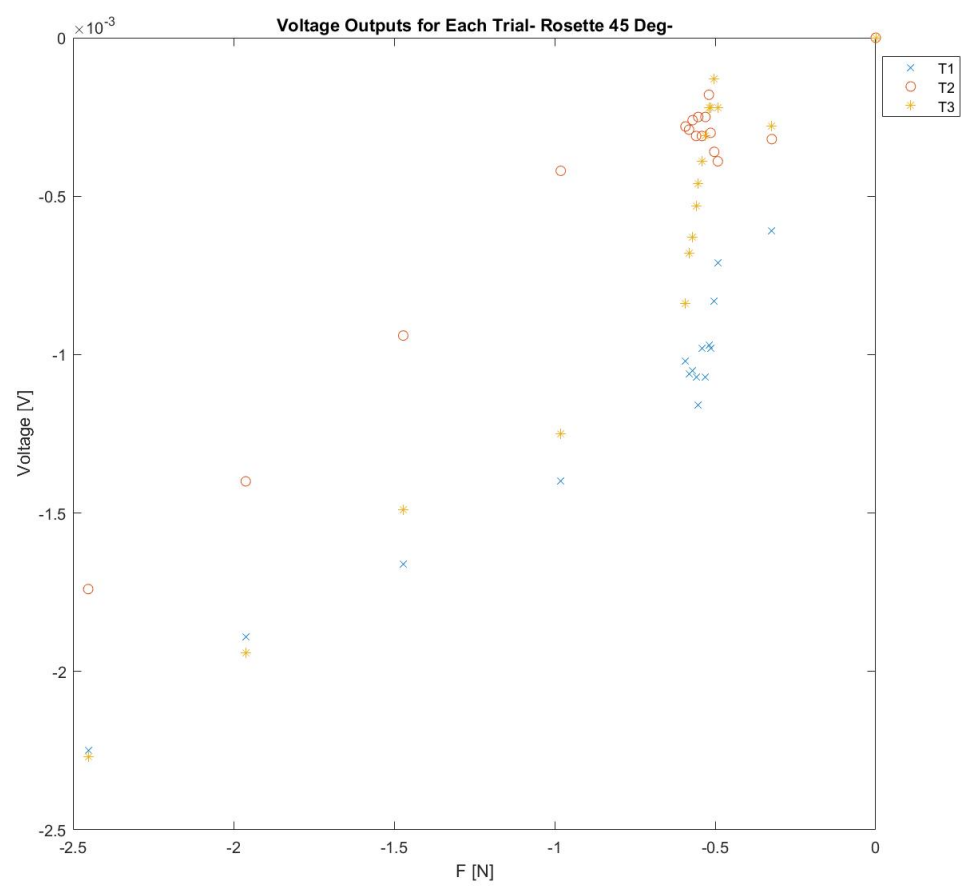

(b) Rosette $45^{\circ}$ (-) Response 


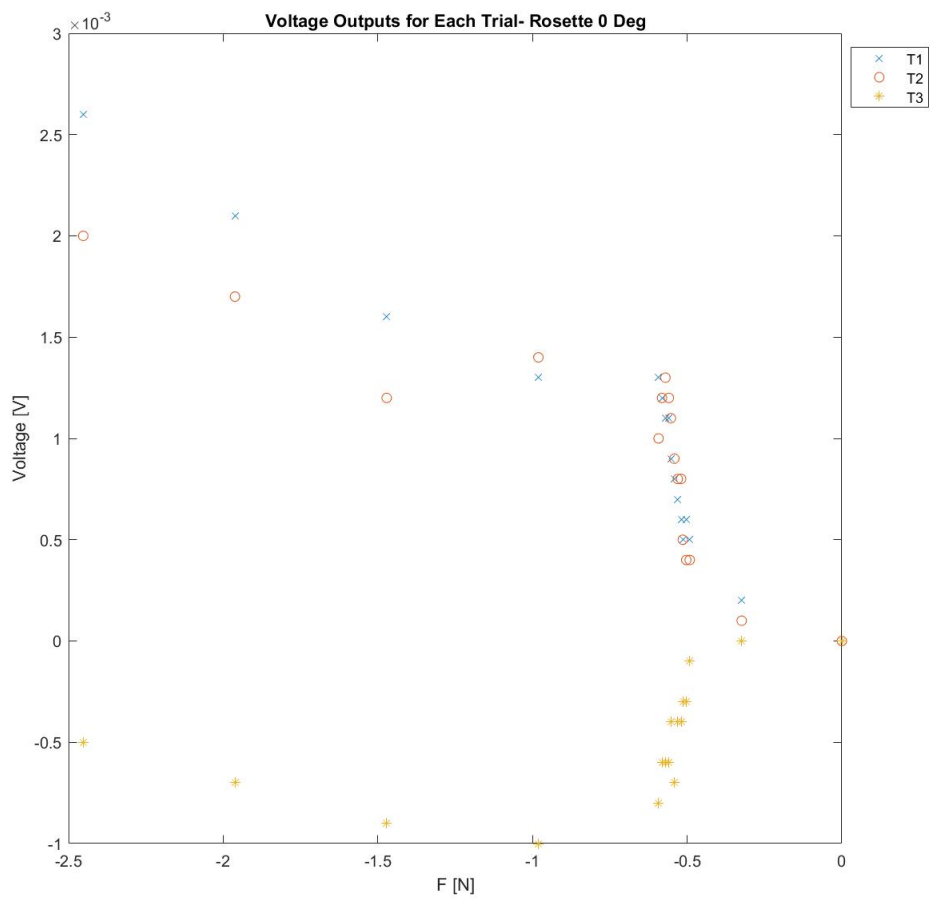

(c) Rosette $0^{\circ}$ Response

Figure 7.10: Voltage Response of All Trials for the Rosette Bridge Outputs at Angle Increment 1 for the $F_{y}$ Load Application 
and moments. Due to the low magnitude of the forces and moments, which the gauges were selected to detect, a poor signal to noise ratio may have impacted the data collected. As previously discussed, this unexpected result could have a significant negative impact on the final prediction model. This result was noted across all trials and loading cases.

Therefore, this stage confirmed that the prototype would not successfully obtain accurate data from the rosette bridge outputs. Changes to the gauge selection would be required to move onto the next stage of prototyping and beam calibration. Linear gauges could be applied to the sides of the sensing beam to instead obtain $F_{x}$ and $F_{y}$. Depending on the bridge setup, to the different loading cases previously described in Section 6.3 , both variables could be obtained. Further instrumentation could also be reviewed to adjust the gain of these bridge outputs. This may result in a response with a lower signal to noise ratio. This stage of prototyping required experimentation to review what type of gauge would be most appropriate for this application. Through the theoretical analysis it was clear that that a low voltage range would be noted from the rosettes. The experimental data validation further confirmed this, but also presented the issues that mounting error and power supply instability had on the system outputs.

Multivariable calibration at this stage would not be effective. The low gauge precision indicated inconsistency across each loading trial, which would not provide an effective approximation of the gauge responses. Therefore, it was determined that the process of completing calibration would be limited to the linear gauge pairs, to assess $F_{z}$ and $M_{x}$ approximation. This would be completed to confirm the form of calibration model that could be applied to the prototype. 


\subsection{Mathematical Calibration Theory}

Chapter 5 outlined the theoretical relationship between the gauge outputs and the effective force and moments applied. However, due to the simplifying assumptions made in the analysis, as well as the mounting error and resultant noise determined throughout the assembly process, the direct application of these equations to determine the user inputs would not provide accurate results, nor be an effective method. Instead, calibration will be required of the sensing beam with the application of a mathematical approach to relate the known inputs with the recorded voltage outputs. It was previously concluded that calibration would not be effective at this stage of the prototype, due to the inaccuracy of the selected gauge rosettes. However, the calibration process was reviewed and tested with the linear gauge results from the validation tests completed.

Instrument calibration requires the development of a mathematical expression which relates the voltage readings from of the sensing beam and the pre-determined calibration inputs. Various mathematical methods exist to relate the known inputs to measured outputs. Through an estimation of the relationship between the inputs and outputs, a calibration model matrix can be developed. To visualize, consider the matrices below. Matrix $A$ represents the values inputted into the experiment system, in this case the voltage recorded across each gauge. Matrix $b$ represents the output of the system, in this application that is the calculated force profile from the keystroke. An estimation model would develop a matrix which reproduces Matrix $A$ given Matrix $b$. 


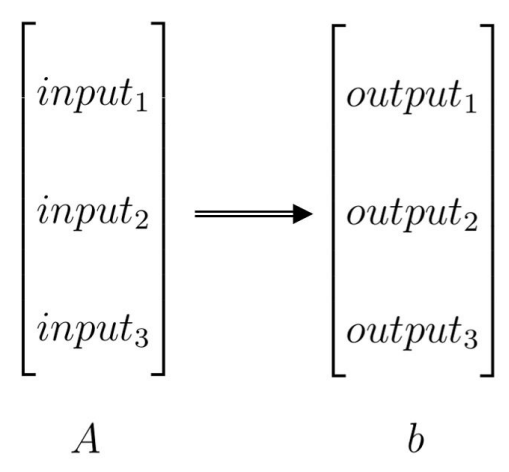

Possible modelling methods exist, in which each method holds its own advantages, often depending on the type of data set to be modelled. More advanced models such as Principal Component Analysis, first examine the structure within the data set to assess their relationship before modelling. The estimation is developed through calculating the principal components. Responses which only appear to contribute to noise are removed before developing the final estimation, which can be achieved through Singular Value Decomposition. Through the assessment of data that contains the most useful information, PCA provides a method to recognize data similarities [73, Section 7.3]. This is different from a least squares approximation, which does not take the pre-existing relationship of the data into consideration before developing an estimation, but rather focusses on predicting the responses. In an application where channel responses are linearly independent, a linear least squares approximation can create an accurate predication. Although various methods exist, like the ones referenced above, they are not appropriate for all testing applications [73, for further details on the models and their application].

As the relationships between the inputs and outputs were already known, a more simplified method could be applied. Preliminary testing confirmed that the gauges had a highly linear response, therefore linear least squares was first considered. Following the theory from [73, Section 4.3], a least squares analysis attempts to solve 
the relationship of $A x=b$. However, an exact solution for $x$ does not exist in this application, as it is an overdetermined system. Instead, an approximation for $x$ is required that can solve the above equation with minimal error. This provides the closest approximation to the exact solution, by developing a solution with the lowest measure of error. A least squares analysis attempts to find the values of the $x$ matrix which minimizes the squared error, in a matrix denoted $\hat{x}$. This matrix can be found mathematically from solving Equation 7.1.

$$
A x=b
$$

therefore,

$$
A^{T} A \hat{x}=A^{T} b
$$

rearranging

$$
\hat{x}=\left(A^{T} A\right)^{-1} A^{T} \cdot b
$$

To visualize this method in the application of the current research, see Appendix I. The estimation of $\hat{x}$ would require an expression to represent the first group of terms in the right-hand side of the equation. This portion of the least squares approximation can be solved through different methods. Development of the relationship of this component of the $\hat{x}$ expression can be solved from processes such as singular value decomposition, QR factorization, or pseudo-inverse analysis [73, for further details on the models]. To determine the most effective approach for this application, the aforementioned methods were assessed experimentally. Initial load testing was completed with the test cantilever beam, previously described in Figure 6.15. A set of pre-measured masses were applied at a known location on the beam to develop a set of bridge voltage data and the pre-determined load input variables. The bridge 
voltages and applied loading conditions were then compared across the possible approximation methods, through $M A T L A B$, to assess their use for the sensing beam application. The comparisons showed that the pseudo-inverse analysis to complete the least squares approximation was effective in accurately relating the data sets. Therefore, this method was reviewed further to apply in the sensing beam application.

\subsubsection{Least Squares Approximation through Pseudo-Inverse Analysis}

The use of pseudo-inverse and least squares approximation does hold limitations. The analysis method selected takes into account all data, to develop a relationship, including any error and irrelevant data. Therefore, in creating the calibration matrix, incorrect assumptions can be made. System noise could even be used in developing the calibration model, as the method attempts to use any variance to identify independence in the matrix data. Although this could provide an accurate prediction when modelled back to the original data, when the calibration matrix is then applied to a new sample of data, it may not be the case. The model assumes that the correlations made in the calibration stage, which were influenced by system noise, also exist in the new sample. In the case of a noisy signal, this would not be the case and would therefore result in an inaccurate model when applying the least squares approximation to new data. This inaccuracy in model becomes more significant in a system in which the data matrices are not linearly independent. The model attempts to find the differences between the data sets, which in a case where the values are nearly collinear, could result in the strong influence on noise or error to distinguish the differences. This consequently, amplifies the impact of the noise and results in an inaccurate model. The data in this application is independent, but not widely 
independent. As the validation of the voltage responses highlighted, the gauges all respond with an increasing voltage to an increasing load application; but this is at different rates depending on the bridge.

At this stage of assessment, a linear least squares approximation is a sufficient choice of estimator model due to the linear response of the data. However, it was clear that this model is sensitive to errors in the system. This could be resolved by reviewing the bridge channel responses at the different loading conditions. As previously defined in Section 6.3, the sensitivity to loading conditions greatly varies with respect to the gauge orientation. Therefore, a bridge pair that does not contribute valuable data to the approximation, could be removed from the calibration set for the specified contact variable which is being considered. The solution to apply the model and remove the known voltage outputs which are irrelevant to the set loading case, would improve the prediction, as a least squares approximation does not screen out data which has a negligible effect on the output response. However, a more complex modelling method could be applied to provide a more thorough analysis. One such method could be the previously described Principal Component Regression method. However, at this stage in the prototype it was determined that this analysis was not worth pursuing until it was confirmed that all desired planes of measurement were perfected.

\section{Execution of Pseudo-Inverse Approximation}

It should again be noted that the data previously collected was intended to simply validate that the measured voltages are related to the applied load. As the rosette data were not accurate, the data were not useable for a full sensor calibration. However, the response of the linear gauge pairs could be applied to evaluate the use of least squares approximation through pseudo-inverse with calibration across the $F_{z}$ loading cases. After a review of the voltage outputs stored, the data collection file was inputted into 
a separate script, available in Appendix J.

First, the data was stored in separate matrices. The first 6 columns, which provided the 6 contact variables for the applied loading case, where stored in a matrix denoted $F$. The voltage outputs across each of the gauge pairs were then stored in a second matrix, $v$. In completing the data collection, every second channel was used to collect the differential output from each bridge, to further avoid channel cross-talk. Therefore, in storing the data the odd columns from $7-15$ were stored in the $v$ matrix. This provided the matrix structure outlined of an input and output matrix, as described previously to set up a least squares approximation.

However, the intent of this evaluation was to only consider the defined $F_{z}$ loading cases. Therefore, the original data were further divided. As outlined in the approximation method, the rows of the matrices should include the experimental trials which are to be considered in the analysis. Therefore, the two matrices were further divided to store the different positions of loading as well as the repeated loading trials. This allowed consideration of which experiments to include in the $F$ and $v$ matrices to evaluate $F_{z}$ and $M_{x}$. It was noted that the repeated trials were required in the data validation stage to evaluate the precision response of the sensing beam. However, in the least squares approximation, the repeated trials were combined to provide the mean voltage at each mass addition for the specified loading position. This provided one mean voltage for each channel output at each loading case.

The process of least squares approximation through a pseudo-inverse analysis was completed following the algebraic procedure outlined in [73, Section 4.3]. The only deviation made from this method was the number of rows of data which were considered, which varied on which experimental trials were used in the stages of calibration analysis. The first step, which was already completed, was to develop the input 
and output matrices which needed to be related. The defined matrices $F$ and $v$ required one additional step prior to completing the pseudo-inverse, outlined in the least squares approximation procedure. To account for the offset of the data, a column of ones was added to the first column of the $v$ matrix. As visually displayed in Appendix I, this meant that the first element of the calibration matrix would represent the coefficient of the total offset. With the correct matrix structure, the pseudo-inverse was then calculated, which provides the solution to the matrix which approximates the relation of the data with minimal error. To assess the accuracy of the approximation, the approximation matrix, which was represented by $q$ in Appendix I and J, was then applied back to the same voltage responses. The allowed a comparison of the original $F$ matrix, with the newly approximated $F$ matrix. A calculation of the percent error gave an initial indication of the success of this method.

To first assess the accuracy of the method, the analysis was applied at each loading position separately. An example of the accuracy in the pseudo-inverse prediction modelled back to the original data can be viewed in Figure 7.11. This provided another indication of the accuracy of the linear gauge pairs. As the previous voltage assessment concluded, the linear gauge pairs responded as expected with the load inputs. This was further reflected when modelling the data back onto itself. Deviations from the expected voltages was noted in the approximation in some spikes in error, see Appendix I. However, a true least squares would need to be applied to a larger set of experimental trials to be fully assessed for its ability to be applied to a future calibration.

After this stage of assessment, the loading positions from each $F_{z}$ case were combined to develop matrices which encompassed all of the relevant experiments. This process was completed for the pre-defined positions 1-8 for the $F_{z}$ loading cases. As 

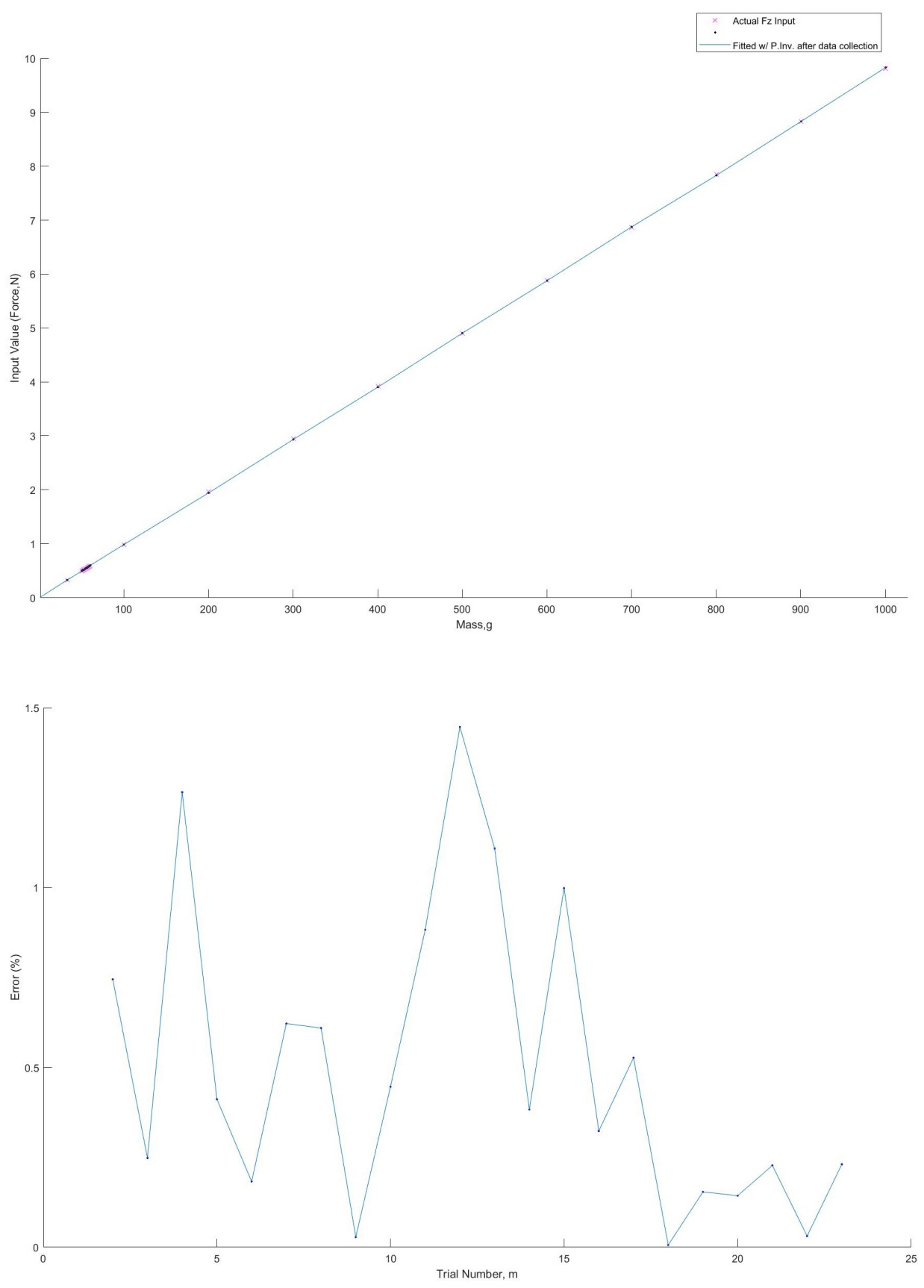

Figure 7.11: Pseudo-Inverse Prediction Modelled Back to the Original Data at Position 1 
previously discussed, the approximation method selected is sensitive to error when collinear inputs are used, as well as when irrelevant data is applied in the calculation. Therefore, the pseudo-inverse was completed with all voltage readings, and then repeated to only select the linear gauge pairs which had been established as accurate and sensitive to the applied loading case. The result from using the linear gauge pairs is displayed in Figure 7.12.

Assessment of the accuracy of this step was made through estimating the applied loading conditions through the use of the calculated matrix and comparing the results with the reference inputs. It was found in the comparison of $F_{z}$ loading cases, this process had a less significant input on the final results. Referring back to data validation of the voltage responses, this result was expected due to the strength of the linear gauge outputs compared to that of the rosettes. The initial approach at the least squares approximation showed the majority of error remained under $3 \%$. The selection of the gauges was more critical for positions in which the gauge responses provided data that was completely irrelevant to the loading, as shown in Appendix I with the $M_{x}$ predictions.

From this initial analysis, a few conclusions can be drawn for future calibration procedures. The least squares approximation provides an accurate prediction from the $F_{z}$ responses. In the assessment of $F_{z}$ loading cases, the least squares approximations should be completed with the voltage outputs from the two linear gauge pairs at Plane $A$ and Plane $B$ of the sensing beam. As previously described in Chapter 5, the linear gauges would be the most sensitive to this bending load. The response of $F_{z}$, with respect to the gauge positions, is dependent on the position of the load application, the positions selected for testing were concluded to be critical in the full analysis. To provide a clear picture of the response of the gauges under 

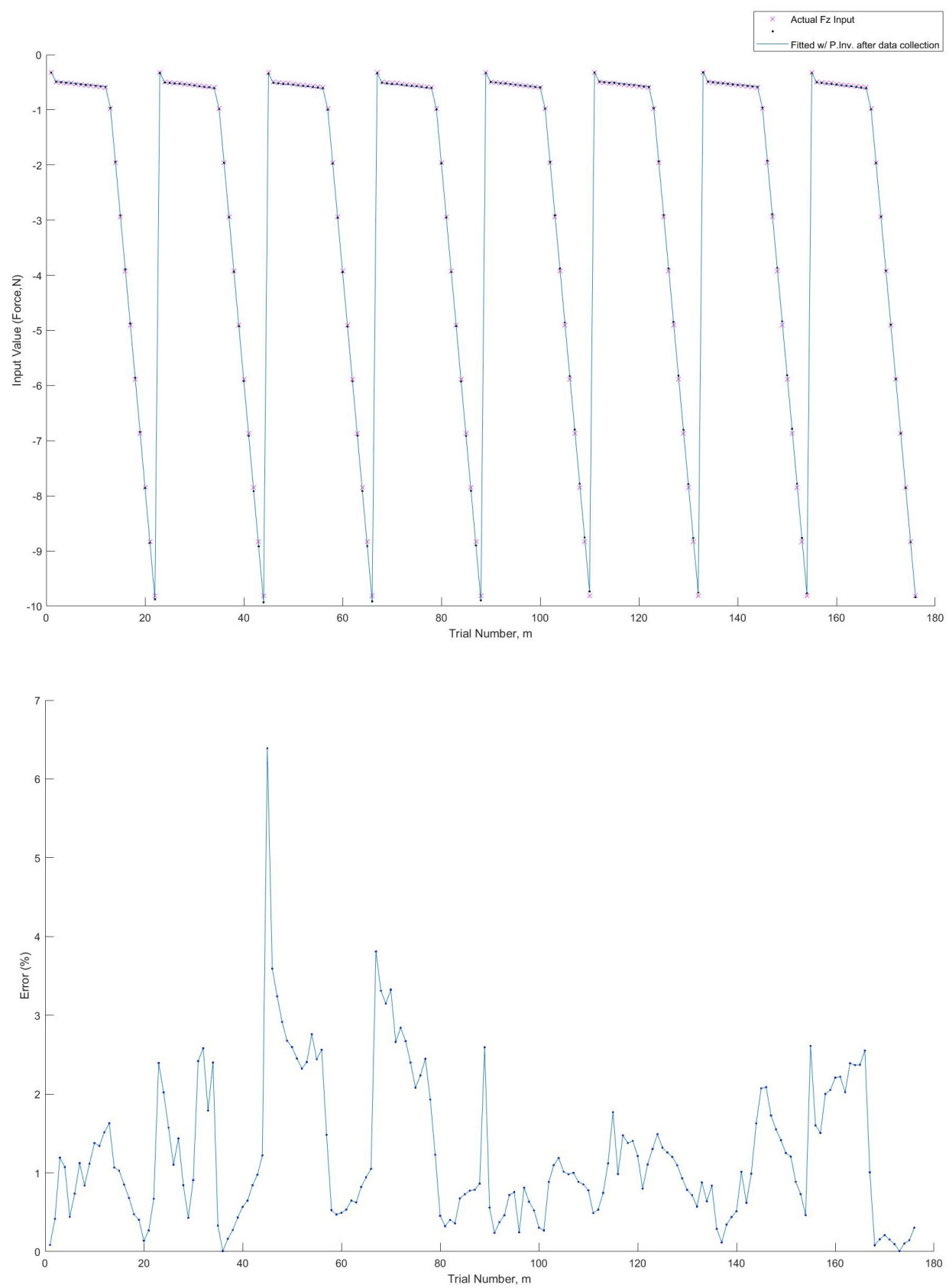

Figure 7.12: Pseudo-Inverse Prediction Modelled Back to the Original Data across all $F_{z}$ Loading Cases with the Voltage Responses from the Linear Gauge Pairs 
different conditions, consideration of the varied loading cases at different positions was necessary. The initial calibration analysis was completed with a high level of accuracy. With the necessary hardware adjustments made to the rosette gauges, it can be assumed that the approximation would be completed equally well with the $F_{x}$ and $F_{y}$ load applications. For the $F_{x}$ loading cases, it would be expected that a linear gauge pair along the side of the beam as well as shear gauges would be utilized, due to the bending and torsional loads. For the $F_{y}$ assessment, the linear gauge pairs from pairs $A 12$ and $B 12$ would again be used. In addition, linear gauges along the side of the beam would be considered for the voltage inputs for the analysis.

Due to the inaccuracy in the rosette responses, this stage of examining calibration options did not assess multivariate analysis, such as the combined $F_{y}$ and $F_{z}$ trials that were completed. This step is critical to complete the full beam calibration. However, significant error exists in rosettes. This means that looking at the ability of least squares analysis to dis-entangle the combined loading responses would not provide a true assessment of the calibration method accuracy. The process would be greatly affected by the current error in the system, which is not expected in the final prototype. After the next stage of prototyping and full calibration, the development of the final matrix $q$ would then be applied in the final data collection script, to be used during user testing. This script, outlined in Appendix F, records the voltages of the gauges during testing and applies the calibration matrix to provide the user input values, solving the previously-described Matrix $F$. 


\subsection{Design Output Parameters and Specifications}

The required design inputs for this thesis were previously outlined in Section 3.2. The overall intent of the research project was to complete multi-axis force measurement with a design that: remains unobtrusive and undetectable to a pianist, is reproducible for future use, and was able to be set up across multiple participants. More specifically, the design inputs were divided into requirements of the key properties and sensor properties.

After completing the data analysis, a comparison of the design inputs and design outputs was made. In general, the design proposed a solution to achieve multi-axis force measurement through a key-embedded sensing solution. It was previously outlined that an accurate multi-axis device was not fully achieved in this design stage. However, the prototype met the design inputs in terms of an unobtrusive and undetectable device. The design selected provided the ability to integrate the prototype key in the piano without additional modification to the surrounding keyboard or piano. The simple design also allowed ease of reproducibility. Therefore, the design met the general design inputs set at the beginning of this study.

The first section of specific design inputs assessed was that of the key geometry and mass properties. The key carried identical outer geometry as the original key. The integrated key sensor fit well within this geometry, which ensured no further modification was required to adjacent keys. The design inputs for the key mass properties were originally set under the assumption that a change in mass properties would have a significant impact on a pianist's ability to maintain their natural performance during future testing. However, the research study, which was summarized in Chapter 4, concluded that the pianist response to mass alterations was not as sensitive as previously assumed. The final mass of the piano key did not deviate significantly 
from the original mass, as a change of less than $2 \mathrm{~g}$ was noted, see Table 7.2. At that point it was determined that the additional mass properties did not need to be fully assessed, as the study outlined that these would not be discernable changes.

The next section of design inputs reviewed was the defined sensor properties. It was clear that the completed measurement tests did not span the full measurement range. At the maximum measurement range tested, it was noted that the voltage outputs were still within the detectable range of the $T \%$. The testing was able to display a change in voltage that was distinguishable with $1 \mathrm{~g}$ increments. These tests were deemed sufficient to test the force measurement range of the gauges.

The resolution was calculated for the linear gauge bridges with reference to the DAQ operating at 16-bits. After dropping the least significant bit, this meant 15bits were used to calculate the resolution. The 15 bit accuracy is over the full $20 \mathrm{~V}$ range, of the LabJack $(-10$ to $+10 \mathrm{~V})$. Therefore, the system is able to detect voltage changes as small a $0.6 \mathrm{mV}$. The validation analysis for the linear gauge pairs saw a voltage range to a maximum of approximately $0.6 \mathrm{~V}$ over a force range of $10 \mathrm{~N}$. This translated to a detectable force of approximately $0.01 \mathrm{~N}$.

The accuracy of the prototype was examined for the $F_{z}$ data validation tests. This was completed through the initial assessment of the calibration methods. The completion of the $F_{z}$ approximation saw percent error data spikes upwards of $8 \%$. However, the majority of error percentage remained below 3\%. In finding the average percent error, it was determined that the error was below $1 \%$. With the adjustments made to the rosettes, it would be expected that a similar range could be met

The sampling frequency of the $T^{r} 7$ was dependent on the number of channels streamed. In this application it was calculated that over the channels used, the 
maximum sampling frequency would be $8333 \mathrm{~Hz}$. This was well above the sampling range originally outlined. As this rate, a smooth and continuous force profile would be recorded. It was noted that future adaptations to the prototype DAQ connection could result in lower sampling speeds. However, a lower rate would still be sufficient for this application. At this stage in prototyping, the highest sampling rate, with the lowest inter-channel delay, was desired to properly assess the gauge responses.

Table 7.2: Final Design Outputs Compared to the Original Design Inputs

\begin{tabular}{|l|l|l|l|}
\hline Metric & Units & Target Value & Output Value \\
\hline \hline Physical Size & $m$ & within key geometry & within key geometry \\
\hline Final Key Mass & $g$ & 81.41 & 79.85 \\
\hline Centre of Mass & $m$ & $(0.02,0.154,0.013)$ & $(0.02,0.149,0.013)$ \\
\hline Force Measurement $\left(F_{z}\right)$ & $N$ & $0.5-60$ & lower range confirmed \\
\hline Force Measurement $\left(F_{y}, F_{x}\right)$ & $N$ & $0-25$ & lower range confirmed \\
\hline Resolution $\left(F_{z}\right)$ & $N$ & 0.01 & 0.01 \\
\hline Accuracy $\left(F_{z}\right)$ & $\%$ error & 1 & $\begin{array}{l}0.98 \% \\
\text { (average from prelim. } \\
\text { analysis })\end{array}$ \\
\hline Sampling Frequency & $H z$ & $500-1000$ & 8333 \\
\hline
\end{tabular}

\subsection{Future Key Integration}

It has been outlined that the prototype key is not at a stage to accurately complete multi-axis sensing. However, final validation from preliminary integration, and key response from user testing of the prototype integrated into the keyboard, could still provide insights for the next stage of design. This was unable to be assessed in the 
scope of this thesis ${ }^{1}$. Initial key integration was intended to further validate the use of the prototype within the keyboard. Possible errors due to key integration were considered. One such possible error was that of electrical interference. In considering the structure of the piano, this was considered unlikely. The brass metal frame of the piano may introduce a shielding effect, but interference noise would not be present. Experimental testing was completed through static testing. Therefore, motion effects were not thoroughly analyzed. However, it was predicted that they would have a negligible impact on the system.

With the current prototype, the following steps would currently be required to integrate the developed key into the place of Key 42 of the Yamaha Disklavier MX1Z piano, in which the key had been originally modelled after. First, to gain access to the full keybed, removal of the front panel, fallboard, and fallboard support would completed. This would provide access to the full keybed, similar to that previously shown in Figure 6.11. After access is gained to the keyboard, the original key 42 would be removed. The prototype key, which holds the same geometric properties, could then be put in place of the original key, by aligning the key with the middle and front balance pins.

Next, with the key in place, the two assembled circuit boards could be placed at the back of the keybed. As previously described, the perfboards were selected as they fit along the base of the keybed underneath the adjacent keys. This would minimize the distance between the perfboard and the sensing beam hardware. As defined in Chapter 6, the wiring from the perfboards were then attached to InAmps at the LabJack expansion board. The ribbon cables would be laid underneath the back of

\footnotetext{
${ }^{1}$ Key Integration was removed from the current stage of research due to the COVID-19 pandemic. The University was closed, and testing with a pianist was not permitted. Therefore, this stage was unable to move forward.
} 
the piano keys along the keybed, to feed to either end of the piano. This was similar to the cable organization of the MIDI instrumentation in the piano, again visible in Figure 6.11. At the bass or treble end of the piano (each had an equally sufficient amount of space), the expansion board would then be placed within the body of the piano. This solution provided the minimal distance between the perfboards and the expansion board, without requiring any adjustments or modifications to be made to any portion of the piano. Finally, the LabJack Tr-Pro and power supply would be placed outside of the piano, at a position where the necessary wiring could be fed through the piano top board to the expansion board. Communication with a host computer to collect data could then be achieved through a usb, ethernet, or wifi connection. This setup could then remain in the piano during the duration of testing, or be removed as necessary. This stage provided a system that was able to remain transportable. 


\section{Chapter 8}

\section{Conclusions and Recommendations}

\subsection{Conclusions}

Previous assessment of the biomechanics of pianist performance has lacked multiaxis force sensing. The research project aimed to discretely collect accurate force and position profiles during a unique keystroke. In this stage of development, this thesis presented a prototype piano key with integrated sensing instrumentation. The design and manufacturing at this stage successfully developed the design of an unobtrusive measurement method that provided the necessary setup to discretely record the applied force and finger contact position during a keystroke. In addition to the required measurements, this thesis outlined additional goals of the key design to be successful in future user testing.

The main goal of this research was to develop a system to complete force measurements of all components of the applied contact force. A second goal of the design was to complete contact position measurement along the surface of the piano key. A novel force and torque integrated key sensor was developed to complete multi-axis force, and position, measurement. The key was designed to maintain key mass properties, remain unobtrusive, and allow continuous measurement during testing. To complete 
measurement, the sensing system consisted of strain gauges which were positioned along the beam to capture normal and shear force, as well as moments, applied to the key. This allowed detection the force components and position of force application on the key surface. Data analysis identified that this stage of prototyping accurately detected the variables of $F_{z}$ and y-position of touch. However, due to the gauge selection and low magnitude of response, the prototype was not able to accurately detect the other elements of the force profile. The current prototype provided the framework for future adaptations to be made to achieve full sensing capabilities.

An additional focus of the research was to ensure the measurement system remained unobtrusive and undetectable to a pianist. Previous approaches to force detection in past literature did not remain unobtrusive to participants, which limited research capabilities and may have had an impact on pianist performance. An initial design goal was to implement a solution which was unobtrusive. This was achieved through integration all sensing instrumentation to be within the piano key. The strain-gauge mounted sensing beam was assembled within the replica key to remain below the keytop surface. Visually, the integrated sensing key appeared unchanged in comparison to the adjacent keys, from the perspective of the pianist. A plastic keytop was selected for this prototype; however, a keytop material which better matches the piano model of interest for future work, could be applied to future prototypes create a visually-undetectable design.

Assessment of pianist perception to key mass alterations was also addressed in this thesis; in reference to developing an undetectable key. Insufficient previous research on this issue currently exists. Therefore, this initial study provided novel conclusions. Four mass alterations were applied across a 3-octave range during participant testing, which varied in total mass and position of placement across the piano keys. The 
majority of participants were unable to identify the mass altered keys; with those who selected correctly focussing on the back-weighted key. Additionally, multiple participants incorrectly selected unmodified keys and identified them as an altered key. The selection, and confidence in selection, of unmodified keys greatly outnumbered the participants who were able to correctly identify the applied changes. The review of these results demonstrated that pianist sensitivity was much lower than previously predicted.

This research held additional goals which were developed with the intent for use of the prototype in future research testing. Testing across various participants required development of a design that did not require modifications to a piano. The completed prototype was assembled to allow all external key electronics to be placed within the piano keybed. To implement the design for future testing, it was also a goal to create a model which could be reproducible. Design selection to place the sensing beam at the front of the piano key has allowed for universal use across multiple keys. The design is independent of the key curvature as well as the variation in key front shape. Therefore, the design could be manufactured and applied across multiple keys, with the additional replica key pieces fabricated. This thesis presented the necessary steps to complete the fabrication and calibration of additional integrated sensing keys. To allow future implementation in research studies, the prototype was required to be user-friendly for testing by non-technical users. At this stage, the prototype was not completely user-friendly. Once installed, the key would not require further adjustment. However, data processing would still require signal collection and processing through the developed programming script. 


\subsection{Contributions}

The completion of this thesis provided new contributions to the research community. One contribution was the completion of the research objective to produce an unobtrusive design. The completed key prototype integrated all measurement and data processing equipment inside the key and the keyboard of the piano, which was not visible to the pianist. As previous literature often required sensor integration on top of the key or to the body of the pianist, this is a novel contribution in this stage of the prototype.

Another contribution was the development of a design to achieve multi-axis force measurement system. This stage of research saw the design and initial fabrication of a prototype to achieve measurement of the full force profile during a keystroke. At this stage, the prototype successfully detected $F_{z}$ and $M_{x}$ (y-position) loading. The design presented a proof of concept for $F_{x}, F_{y}$, and $M_{y}$ measurement. With the recommended changes to the instrumentation, successful multi-axis measurement with a high degree of accuracy is achievable. This level of data collection is novel in the research community, as previous measurement methods only considered 1-axis force measurement. The design outline for the next stage of prototyping has been outlined to achieve multi-axis sensing, using the developed design and instrumentation recommended. This development will provide new research opportunities, and new insights in the biomechanics of pianist movement.

This thesis also saw the completion of a piano key touch variation study. The conclusions of this study provided new insights on pianist perception to key touch and

physical key variations. Researchers originally believed a high sensitivity to any key weight changes was present amongst pianists. However, this research demonstrated that the sensitivity was not only lower than previous studies had recorded, but were 
also more dependent on the properties of the centre of mass and mass moment of inertia change rather than strictly on the total mass. The findings of the study have set the framework for future research.

\subsection{Recommendations and Future Work}

This thesis presented the hardware design for the ongoing research to develop a user-friendly multi-axis piano key sensor. Further adaptations and iterations are required to reach the final research goal. These next steps are outlined in the below sections.

\section{Sensor Modifications}

Chapter 7 extensively outlined the necessary design changes required to improve the accuracy in the detection of the remaining contact variables. As outlined, the current linear strain gauges are functional to measure $F_{z}$ and $M_{x}$ contact variables with high precision. The rosettes were functional in measuring $F_{x}$ and $M_{y}$, but with low precision. Due to the low magnitude in response the rosettes were unable to accurately detect $F_{y}$. The use of the gauge rosettes impacted the ability to accurately complete multi-axis measurements. A more effective strain gauge arrangement could be implemented to improve the accuracy of these responses. Instead of the rosette arrangement, an additional pair of linear gauges could be affixed to detect $F_{y}$ and $M_{y}$ force application. Shear detection, for $F_{x}$, could be achieved through shear gauges, rather than a stacked rosette, dependent on the size constraints of gauges.

Additionally, gauges with a higher nominal resistance could be implemented to further increase the voltage output. Use of gauges with a higher nominal resistance would also permit a higher excitation voltage to be applied. In relation to this, a more 
stable power supply could be acquired to limit the signal drift across the circuitry. Completion of these modifications are necessary to achieve a higher accuracy across all components of the force measurement profile. These recommendations would further distinguish the small force components from the system noise.

\section{Further Investigate Data Analysis Methods}

One element of data analysis that could be further assessed would be the change in accuracy when including the power supply in the data analysis for each bridge. This could be achieved by normalizing the channel outputs with respect to the bridge power. This would also allow assessment of better measures of repeatability to be made. Chapter 7 additionally provided an overview of the necessary steps to complete calibration of the sensor data, once the instrumentation changes have been implemented. A future consideration would be to evaluate the impact multivariate calibration has on the estimated keystroke force variables. The least squares approximation applied to calibrate the key was effective in the initial stage of data analysis due to the linear response of the data. However, further examination of multivariate calibration options is recommended. The steps outlined for the Linear Least Squares Approximation could be applied and compared to a more involved method such as the previously suggested Principal Component Regression. PCR, which assesses the structure of the data to be calibrated, could be used to further filter out irrelevant and noisy data from the calibration, rather than assessing it manually. This would help to develop a strong calibration model.

\section{Improve Robust Design}

The next stage of prototyping could be further modified to achieved a more robust and "user-friendly" design. The development of a fully-packaged system would allow easier implementation into a variety of future studies. The system should be able to 
withstand frequent transportation and manipulation by multiple users. At current prototype is fully portable, but still requires some knowledge of electronics when handling. The electronics system could be further refined by replacing the perfboards with a printed circuit board (PCB). A PCB would not only provide a more robust design, but would also allow simplified manufacturing to reproduce the circuitry across multiple keys.

To further improve the ease of integration in the piano, development of a wireless method could additionally be introduced. Wireless telemetry would not only provide a more streamlined system, but would also improve the "user-friendly" aspect of the prototype key, as less assembly of electronics and DAQ components would be required. These options were assessed in the prototyping stage; however, to ensure a full proof of concept and validation of the model, they were not implemented in this stage. The data processing at this stage required running a $M A T L A B$ script to complete data collection. Another future consideration would be to create a more user-friendly front-end interface for data collection and display. This would allow a wider range of research use for a different fields of musician movement studies.

\section{Continue User Testing}

In regards to the touch variation experiments, it is recommended that further testing be completed. The current stage of research was limited to the small sample size and small number of key alterations. Testing with a larger sample size, with testing groups of varying degrees of experience, could provide further insight. Further key alterations, including higher percent weight changes, could also be assessed.

As previously discussed, user testing was unable to move forward in this stage of research. It is recommended that the key prototype be integrated into the piano to 
allow user testing to be completed. Data collection during assigned playing tasks would allow further design validation, as well as initial insight into the components of force applied during a pianist keystroke.

\section{Implementation of Instrumented Key into Ongoing Musician Studies}

The intent of the key prototype was to provide an unobtrusive measurement method for pianist keystroke force measurement. After development of the final instrumented key, the next step would be to integrate the key in the piano for future testing. The key could be used to provide measurement in ongoing studies, such as those assessing the biomechanics of pianist performance, and the variation in keystroke force application between skilled pianists. 


\section{List of References}

[1] A. A. Reblitz, Piano Servicing, Tuning, and Rebuilding: For the Professional, the Student, and the Hobbyist. Lanham, Maryland: Vestal Press, Inc., 2nd ed., 1993.

[2] "Polymers in the Piano." https://pslc.ws/welcome/tour/polycons/piano. htm? [Online; accessed 20-February-2020].

[3] A. Thorin, X. Boutillon, J. Lozada, and X. Merlhiot, "Non-smooth dynamics for an efficient simulation of the grand piano action," pp. 1-18, 2017.

[4] W. Everett, The Foundations of Rock: From "Blue Suede Shoes" to "Suite: Judy Blue Eyes”. Oxford University Press, USA, 2008.

[5] "Types of Woodworking Joints without screws." http://labois.com/en/ woodworking/types-woodworking-joints-without-screws/. [Online; accessed 04-May-2020].

[6] "Connection Elements." https://www.boschrexroth.com/en/xc/products/ product-groups/assembly-technology/basic-mechanic-elements/ connection-elements. [Online; accessed 04-May-2020].

[7] DurhamInstruments, "RF9 Miniature Rosette." https://disensors.com/ product/rf9/. [Online; accessed 02-December-2019]. 
[8] M. Critchley and R. Henson, eds., Music and the Brain. Butterworth-Heinemann, 1st ed., 1977.

[9] C. Zaza, C. Charles, and A. Muszynski, "The Meaning of Playing-Related Musculoskeletal Disorders to Classical Musicians," Social Science and Medicine, vol. 47, no. 12, pp. 2013--2023, 1998.

[10] C. Zaza, "Playing-related musculoskeletal disorders in musicians: a systematic review of incidence and prevalence," Canadian Medical Association. Journal, vol. 158, no. 8, 1998 .

[11] I. Winspur, C. Parry, and B. Wynn, "The Musician's Hand," Journal of Hand Surgery, vol. 22, no. 4, pp. 433-440, 1997.

[12] C. Guptill and M. Bruijn, "Case study: Musicians' playing-related injuries," Work, vol. 30, pp. 307-310, 2008.

[13] H. J. Fry, "The treatment of overuse syndrome in musicians. Results in 175 patients," Journal of the Royal Society of Medicine, vol. 81, no. 10, pp. 572-575, 1988.

[14] P. Bragge, A. Bialocerkowski, and J. McMeeken, "A systematic review of prevalence and risk factors associated with playing-related musculoskeletal disorders in pianists," Occupational Medicine, vol. 56, no. 1, pp. 28-38, 2006.

[15] L. M. Kok, B. M. Huisstede, V. M. Voorn, J. W. Schoones, and R. G. Nelissen, "The occurrence of musculoskeletal complaints among professional musicians: a systematic review," International Archives of Occupational and Environmental Health, vol. 89, no. 3, pp. 373-396, 2016.

[16] J. Gat, The Technique of Piano Playing. London, England: Collet's Holding, 2nd ed., 1965. 
[17] O. Ortmann, The Physical Basis of Piano Touch and Tone. 1925.

[18] W. Goebl, R. Bresin, and A. Galembo, "Touch and temporal behavior of grand piano actions," The Journal of the Acoustical Society of America, vol. 118, no. 2, pp. 1154-1165, 2005.

[19] A. Askenfelt and E. V. Janssen, "From touch to string vibrations. I: Timing in the grand piano action," Journal of the Acoustical Society of America, vol. 88, no. 1, pp. 52-63, 1990.

[20] E. N. Schachter and I. Kolcic, "Health Problems in Musicians A Review," Acta Dermatovenerologica Croatica, vol. 13, no. 4, pp. 247-251, 2005.

[21] S. Sheibani-Rad and J. J. Wolfe, "Hand disorders in musicians: The orthopaedic surgeon's role," The Bone and Joint Journal, 2013.

[22] B. Ramazzini, "De Morbis Artificum Diatriba [ Diseases of Workers ]," vol. 91, no. 9, pp. 1380-1382, 1713.

[23] A. Szanto, "Orpheus wounded : The experience of pain in the professional worlds of the piano," Theory and Society, vol. 25, pp. 1-44, 1996.

[24] J. Davies and S. Mangion, "Predictors of Pain and Other Musculoskeletal Symptoms among Professional Instrumental Musicians: Elucidating Specific Effects," Medical Problems of Performing Artists, vol. 17, pp. 155--168, 2002.

[25] L. K. et al.

[26] V.A.E. Baadjou et al., "Systematic review: risk factors for musculoskeletal disorders in musicians," Occupational Medicine, vol. 66, no. 8, pp. 614-622, 2016. 
[27] H. M. Paarup, J. Baelum, J. W. Holm, C. Manniche, and N. Wedderkopp, "Prevalence and consequences of musculoskeletal symptoms in symphony orchestra musicians vary by gender: A cross-sectional study," BMC Musculoskeletal Disorders, vol. 12, no. 1, p. 223, 2011.

[28] H. Blackie et al., "An Investigation of Injury Prevention among University Piano Students," Medical Problems of Performing Artists, vol. 14, pp. 141--149, 1999.

[29] R. Parncutt, "Can researchers help artists? Music performance research for music students," Music Performance Research, vol. 1, no. 1, pp. 13-50, 2007.

[30] D. Russell, "Establishing a biomechanical basis for injury preventative piano pedagogy," Recherche en education musicale, vol. 24, pp. 105-117, 2006.

[31] D. C. Harding, K. D. Brandt, and B. M. Hillberry, "Minimization of Finger Joint Forces and Tendon Tensions in Pianists," Medical Problems of Performing Artists, pp. 103-108, 1989.

[32] C. Guichard, "Marie Jaell: The Magic Touch, Piano Music by Mind Training," in Algora Publishing, pp. 145-161, 2004.

[33] J. MacRitchie, "The art and science behind piano touch: A review connecting multi-disciplinary literature," Musicae Scientiae, vol. 19, no. 2, pp. 171-190, 2015.

[34] I.-S. Chung, J. Ryu, N. Ohnishi, B. Rowen, and J. Headrich, "Wrist Motion Analysis in Pianists," Medical Problems of Performing Artists, pp. 1-5, 1992.

[35] F.J Bejjani et al., "Comparison of Three Piano Techniques as an Implementation of a Proposed Experimental Design," Medical Problems of Performing Artists, pp. 109-113, 1989. 
[36] J. MacRitchie and N. J. Bailey, "Efficient Tracking of Pianists' Finger Movements," Journal of New Music Research, vol. 42, no. 1, pp. 79-95, 2013.

[37] D. C. Harding, K. D. Brandt, and B. M. Hillberry, "Finger joint force minimization in pianists using optimization techniques," Journal of Biomechanics, 1993.

[38] A. Thorin, X. Boutillon, and J. Lozada, "Modelling the dynamics of the piano action : is apparent success real ?," Acta Acustica united with Acustica, Hirzel Verlag, pp. 1-10, 2014.

[39] D. Parlitz, T. Peschel, and E. Altenmuller, "Assessment of dynamic nger forces in pianists: Effects of training and expertise," Journal of Biomechanics, vol. 31, pp. 1063-1067, 1998.

[40] D. Parlitz, W. Trappe, D. Drescher, and E. Altenmuller, "Experimental Setup for a Combined Motion, Force and Sound Intensity Analysis of Professional Piano Technique,"

[41] H. Riehle, R. Fritsch, and H. Gärtner, "A Dynamometric Analysis of Piano Playing," in International Society of Biomechanics in Sports, 1998.

[42] H. Kinoshita, S. Furuya, T. Aoki, and E. Altenmüller, "Loudness control in pianists as exemplified in keystroke force measurements on different touches," The Journal of the Acoustical Society of America, vol. 121, no. 5, pp. 2959-2969, 2007.

[43] S. Furuya and H. Kinoshita, "Expertise-dependent modulation of muscular and non-muscular torques in multi-joint arm movements during piano keystroke," Neuroscience, vol. 156, no. 2, pp. 390-402, 2008. 
[44] S. Furuya, R. Osu, and H. Kinoshita, "Effective utilization of gravity during arm downswing in keystrokes by expert pianists," Neuroscience, vol. 164, no. 2, pp. $822-831,2009$.

[45] S. Furuya, E. Altenmüller, H. Katayose, and H. Kinoshita, "Control of multi-joint arm movements for the manipulation of touch in keystroke by expert pianists," BMC Neuroscience, vol. 11, no. July, 2010.

[46] S. Furuya and E. Altenmüller, "Flexibility of movement organization in piano performance," Frontiers in Human Neuroscience, vol. 7, no. July, pp. 1-10, 2013.

[47] S. Furuya, M. Flanders, and J. F. Soechting, "Hand kinematics of piano playing," Journal of Neurophysiology, vol. 106, no. 6, pp. 2849-2864, 2011.

[48] T. Oku and S. Furuya, "Skilful force control in expert pianists," Experimental Brain Research, vol. 235, no. 5, pp. 1603-1615, 2018.

[49] T. Grosshauser, C. Helveticum, B. Tessendorf, G. Tröster, H. Hildebrandt, V. Candia, and Z. Zürich, "Sensor Setup for Force and Finger Position and Tilt Measurements for Pianists," Smc 2012, 2012.

[50] T. Grosshauser and G. Troster, "Musical instrument interaction : Development of a sensor fingerboard for string instruments," in International Conference on Tangible, Embedded and Embodied Interaction, pp. 177-180, 2014.

[51] T. Grosshauser and G. Tröster, "Finger Position and Pressure Sensing Techniques for String and Keyboard Instruments," Proceedings of the International Conference on New Interfaces for Musical Expression, pp. 479-484, 2013.

[52] M. Flückiger, T. Grosshauser, and G. Tröster, "Precision Finger Pressing Force Sensing in the Pianist-Piano Interaction," 13th Sound and Music Computing Conference (SMC 2016), no. November, pp. 137-142, 2016. 
[53] A. P. McPherson, "Portable Measurement and Mapping of Continuous Piano Gesture," Proceedings of the International Conference on New Interfaces for Musical Expression, pp. 152-157, 2013.

[54] A. Mcpherson and Y. Kim, "Augmenting the Acoustic Piano with Electromagnetic String Actuation and Continuous Key Position Sensing," in Conference on New Interfaces for Musical Expression, no. Nime, pp. 217-222, 2010.

[55] A. McPherson, "TouchKeys: Capacitive Multi-Touch Sensing on a Physical Keyboard," Proceedings of the International Conference on New Interfaces for Musical Expression, pp. 1-4, 2012.

[56] A. McPherson, "Buttons, handles, and keys: Advances in continuous-control keyboard instruments," Computer Music Journal, vol. 39, no. 2, pp. 28-46, 2015.

[57] R. A. Moog and T. L. Rhea, "Evolution of the keyboard interface. The Bosendorfer 290 SE recording piano and the Moog Multiply-Touch-Sensitive keyboards," Computer Music Journal, vol. 14, no. 2, pp. 52-60, 1990.

[58] R. Lamb, The Seaboard: discreteness and continuity in musical interface design. PhD thesis, 2014.

[59] E. Hayashi, M. Yamane, and H. Mori, "Behavior of piano-action in a grand piano. I. Analysis of the motion of the hammer prior to string contact," Journal of the Acoustical Society of America, vol. 105, no. 6, 1999.

[60] D. G. Alciatore and M. B. Histand, Introduction to Mechatronics and Measurements Systems. New York City, New York: McGraw-Hill, 4th ed., 2012.

[61] R. C. Juvinall and K. M. Marshek, Fundamentals of Machine Component Design. Hoboken, NJ: Wiley, 5th ed., 2012. 
[62] M. Fluckiger, T. Grosshauser, and G. Troster, "Evaluation of Piano Key Vibrations Among Different Acoustic Pianos and Relevance to Vibration Sensation," IEEE Transactions on Haptics, vol. 11, no. 2, pp. 212-219, 2018.

[63] R. Hibbeler, Engineering Mechanics: Dynamics. Upper Saddle River, New Jersey: Pearson, 13th ed., 2013.

[64] Asserin, J et al., "Measurement of the friction coefficient of the human skin in vivo: Quantification of the cutaneous smoothness," Colloid and Surfaces B: Biointerfaces, vol. 19, no. November, pp. 1-12, 2000.

[65] H. Järveläinen, S. Papetti, S. Schiesser, and T. Grosshauser, "Audio-Tactile Feedback in Musical Gesture Primitives: Finger Pressing," Proceedings of the Sound and Music Computing Conference 2013, pp. 109-114, 2013.

[66] S. V. Kochetkova, N. A. Kolganova, E. N. Timofeev, and V. L. Florent'ev, "Evaluation of a Digital Grand Piano for Vibrotactile Feedback Experiments and Impact of Finger Touch on Piano Key Vibrations," Bioorganicheskaia khimiia, vol. 34, no. 4, pp. 506-512, 2008.

[67] A. Askenfelt and E. V. Jansson, "On Virbation Sensation and Finger Touch in Stringed Instrument Playing," Music Perception: An Interdisciplinary Journal, vol. 9, no. 3, pp. 311-349, 1992.

[68] O. Ortmann, "Weight Discrimination as a Measure of Technical Skill in Piano Playing," Journal of Comparative Psychology, vol. 3, no. 1, pp. 11-26, 1923.

[69] P. Ragert, A. Schmidt, E. Altenmüller, and H. R. Dinse, "Superior tactile performance and learning in professional pianists: Evidence for meta-plasticity in musicians," European Journal of Neuroscience, vol. 19, no. 2, pp. 473-478, 2003. 
[70] M. Hosoda and S. Furuya, "Shared somatosensory and motor functions in musicians," Scientific Reports, vol. 6, no. November, pp. 1-10, 2016.

[71] D. Stanwood, "Down Weight versus Equation Piano Key Balancing: Shifting the Paradigm," Piano Technicians Guild Journal, no. June, 2014.

[72] L. Prichard and V. Barwick, "Preparation of Calibration Curves A Guide to Best Practice," tech. rep., 2003.

[73] G. Strang, Introduction to Linear Algebra. Wellesley-Cambridge Press, 5th ed., 2016. 


\section{Appendix A}

\section{Piano Key Physical Properties}

\section{A.1 Mass Properties}

The mass, mass moment of inertia, and centre of mass of the piano key were measured. The final values are provided in Table A.2. The mass of the key was measured using a digital precision lab scale. Determination of centre of mass was completed experimentally through plumb line testing. As outlined in Figure A.1, the test consists of suspending a body by a string with a weighted end, and marking a line at the strings suspended position. The test is then repeated at another point along the body. The intersection of those points provides the centre of mass along that plane.
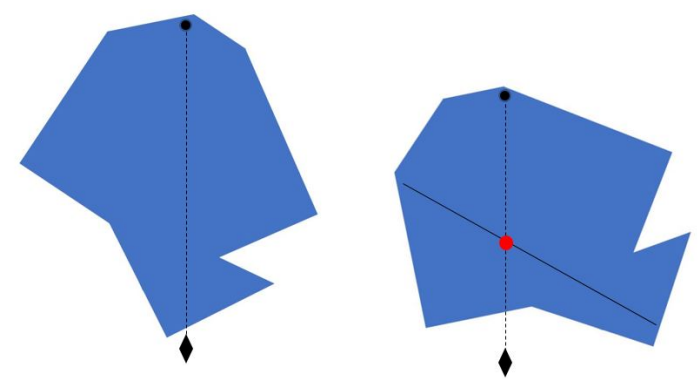

Figure A.1: Experimental Determination of Centre of Mass Through Plumb Line Intersection 
The mass moment of inertia was additionally determined experimentally, due to the complex shape and material components of the piano key [63, for background theory]. Pendulum testing was completed to relate the period of oscillation and moment of inertia of the body. The mass moment of inertia was determined through allowing the suspended body to oscillate. The timed oscillations were about a pivot point I, which were determined through Equation A.1, with reference to the experimental setup shown in Figure A.2.

$$
I=\frac{m g D^{2} T^{2}}{16 \pi^{2} h}
$$

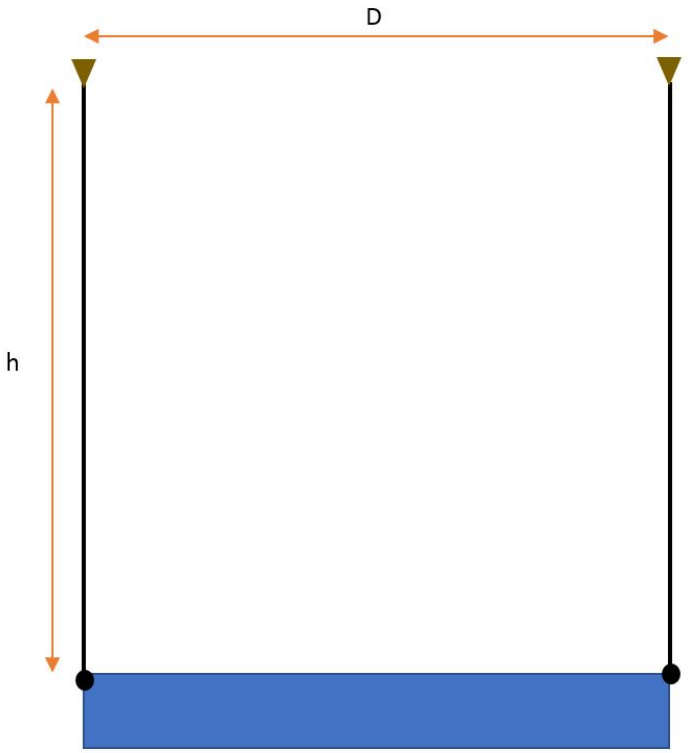

Figure A.2: Experimental Determination of Mass Moment of Inertia Through Pendulum Testing

The experimental setup assumed the mass of the string and attachment screws to the body were negligible. It additionally assumed that rotation only occurred in 1-axis. Before completing testing of the piano key, verification of the experimental setup was first required. A wooden plank was used for this set, shown in Figure 
A.3. Time oscillations were completed over 5 trials, after which the average period was determined. The time was then applied to the below equations, to confirm the experimental gravity. The test showed in error of under $2 \%$, which confirmed the setup was effective to complete testing of the piano key.

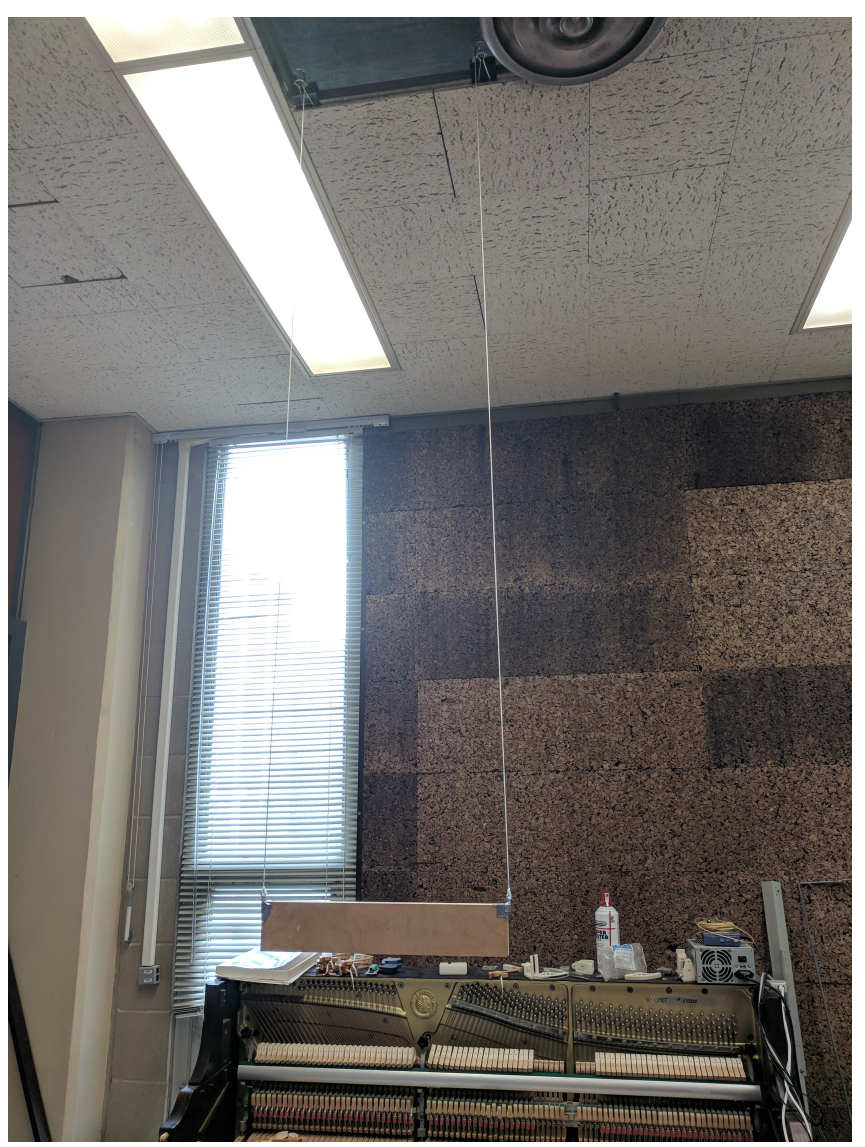

Figure A.3: Verification of Pendulum Testing

$$
\omega=2 \pi f
$$

expanded,

$$
\omega=\frac{2 \pi}{T}
$$


as,

$$
\omega=\sqrt{\frac{g}{h}}
$$

substitued and rerranged,

$$
\begin{gathered}
g=\omega^{2} h \\
g=\frac{4 \pi^{2} h}{T^{2}}
\end{gathered}
$$

Testing was then completed to experimentally determine $I_{z}$ and $I_{y}$. A total of 5 trials were completed for each test, in which the time for 7 oscillations were timed. The average period of each trial was then computed and inputted into Equation A.1. An example of the arrangement for the determination of $I_{z}$ can be viewed in Figure A.4. An example of this experimental process for $I_{z}$ is provided below.

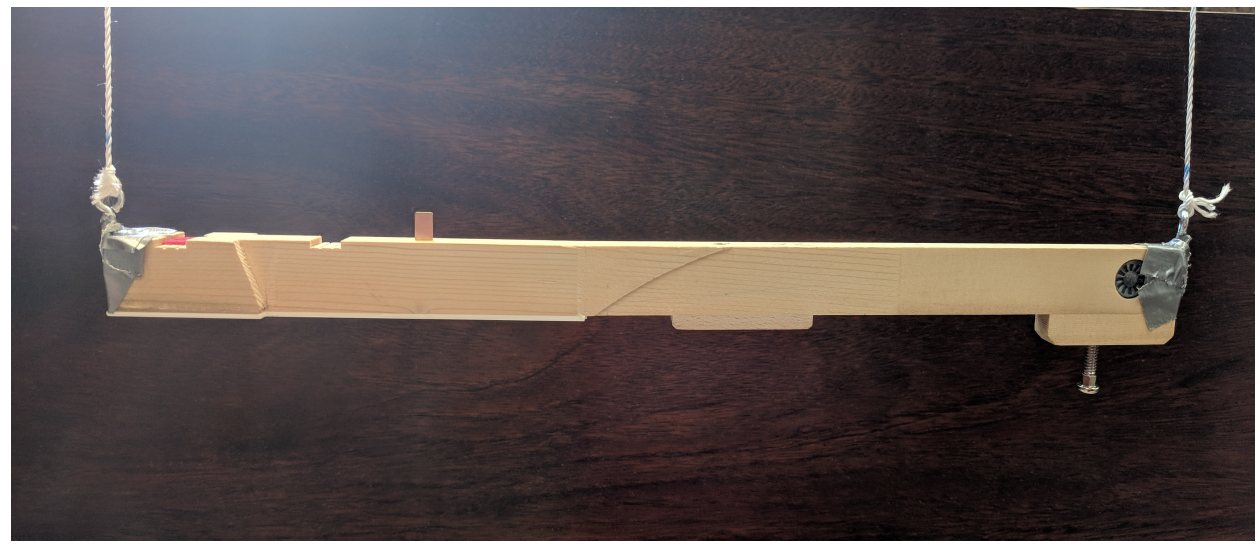

Figure A.4: Experimental Determination of $I_{z}$ 


\section{Example of Raw Data from Pendulum Testing}

Table A.1: Experimental Data from $I_{z}$ Pendulum Testing

\begin{tabular}{|c|c|c|}
\hline Trial & Time for 7 Oscillations (sec) & Average Period (sec) \\
\hline 1 & 12.46 & 1.78 \\
\hline 2 & 12.26 & 1.75 \\
\hline 3 & 12.33 & 1.76 \\
\hline 4 & 12.36 & 1.77 \\
\hline 5 & 12.11 & 1.73 \\
\hline Average $(\mathrm{T})$ & - & 1.76 \\
\hline
\end{tabular}

$$
I=\frac{m g D^{2} T^{2}}{16 \pi^{2} h}
$$

where,

$\mathrm{m}=81.41 \mathrm{~g}$

$\mathrm{g}=9.81 \mathrm{~m} / \mathrm{s}^{2}$

$\mathrm{D}=0.37 m$

$\mathrm{h}=1.551 \mathrm{~m}$

therefore,

$\mathrm{I}=1.38 \times 10^{-3} \mathrm{~kg} \cdot \mathrm{m}^{2}$

Final Mass Properties of Piano Key 42

Table A.2: Mass Properties of Piano Key 42

\begin{tabular}{|l|l|lr|lr|l|l|}
\hline Component & Mass (kg) & $\begin{array}{l}\text { Centre of } \\
\text { Mass, } \\
(\mathrm{m})\end{array}$ & $\begin{array}{l}\text { Centre of } \\
\text { Mass, y } \\
(\mathrm{m})\end{array}$ & $\begin{array}{l}\text { Moment of } \\
\text { Inertia, I } \\
\left(\mathrm{kg} \cdot \mathrm{m}^{2}\right)\end{array}$ & $\begin{array}{l}\text { Moment of } \\
\text { Inertia, I } \\
\left(\mathrm{kg} \cdot \mathrm{m}^{2}\right)\end{array}$ \\
\hline $\begin{array}{l}\text { Piano Key } \\
42\end{array}$ & 0.08141 & 0.02 & 0.154 & $1.38 \times 10^{-3}$ & $1.40 \times 10^{-3}$ \\
\hline
\end{tabular}




\section{A.2 Geometric Properties}

A 3D scan of the piano key was completed with an Artec Space Spider, which is a high-resolution 3D scanner. The scanner, shown in Figure A.5, was used to complete four scans of the piano key to ensure the full key geometry was obtained. Scans were then reviewed to remove outlying geometry, and were aligned to produce one full body object, as shown in Figure A.6.

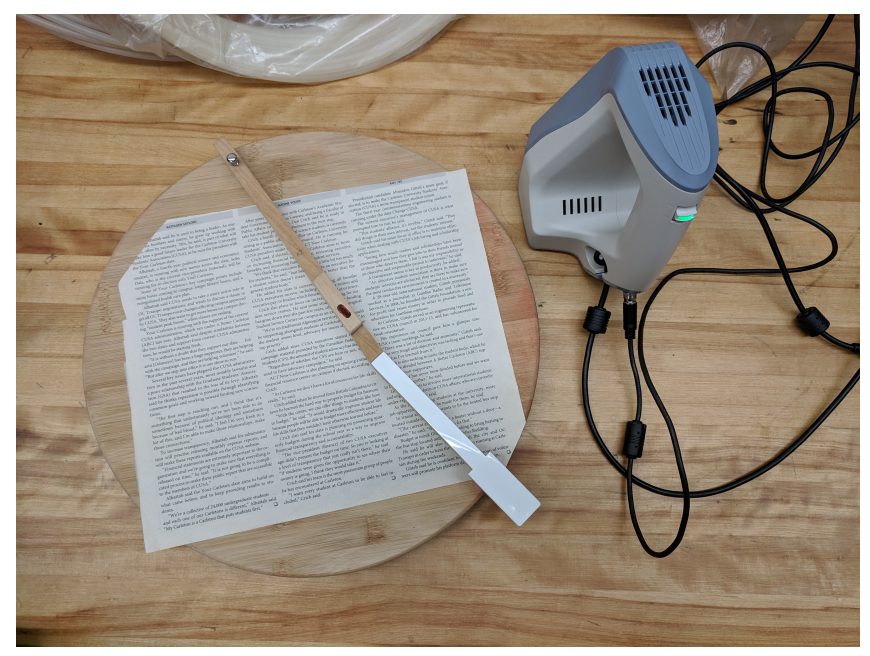

Figure A.5: Setup to complete 3D Scan of a Piano Key

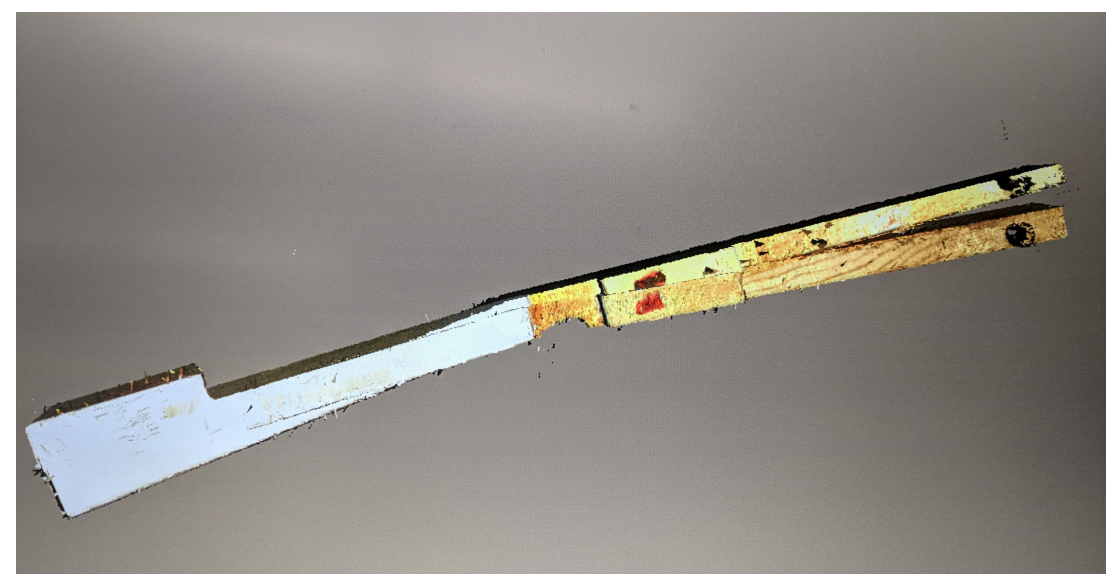

Figure A.6: Alignment of Key Scans to Produce a Final Model 
After alignment was completed, fusion and confirmation of final geometry was completed. This produced a final mesh which was saved as an STL file. The STL file was then imported into PTC Creo where the mesh was traced to produce the final model for further editing to meet the prototype designs. Dimensions were compared with those physically measured the accuracy of the scan. 


\section{Appendix B}

\section{Commercial Sensors Assessed}

Table B.1: Selection of Commercial Sensors Reviewed

\begin{tabular}{|c|c|c|c|c|c|}
\hline Model & Туре & $\begin{array}{l}\text { Manufac- } \\
\text { turer }\end{array}$ & Size $(\mathrm{mm})$ & Mass (g) & $\begin{array}{l}\text { Rated Capacity } \\
(\mathrm{N})\end{array}$ \\
\hline R04 S-Beam & 1-axis & Checkline & $\begin{array}{l}\mathrm{L}=21.6, \\
\mathrm{~W}=19.1, \\
\mathrm{H}=7.6\end{array}$ & 7 & up to 500 \\
\hline FSG Series & 1-axis & Honeywell & $\begin{array}{l}\mathrm{L}=12.7 \\
\mathrm{~W}=7.98 \\
\mathrm{H}=10.3\end{array}$ & $\mathrm{n} / \mathrm{a}$ & 20 \\
\hline LMB-A-200N & 1 -axis & Kyowa & $\begin{array}{l}\mathrm{D}=10 \\
\mathrm{~L}=10+18 \\
\mathrm{H}=4\end{array}$ & 15 & 200 \\
\hline LMBT-A-200N & 1 -axis & Kyowa & $\begin{array}{l}\mathrm{D}=10 \\
\mathrm{~L}=10+10 \\
\mathrm{H}=4\end{array}$ & 15 & 200 \\
\hline
\end{tabular}




\begin{tabular}{|c|c|c|c|c|c|}
\hline KM25 & 1 -axis & $\begin{array}{l}\text { Me- Mebeta } \\
\text { System }\end{array}$ & $\mathrm{D}=25, \mathrm{H}=12$ & 17 & 200 \\
\hline KM26 & 1 -axis & $\begin{array}{l}\text { Me- Mebeta } \\
\text { System }\end{array}$ & $\begin{array}{l}\mathrm{D}=25.4, \\
\mathrm{H}=11\end{array}$ & 80 & 200 \\
\hline LCKD/LCMKD & 1 -axis & Omega & $\mathrm{D}=9.6, \mathrm{H}=3$ & 14 & 200 \\
\hline $209 \mathrm{C} 11$ & 1 -axis & $\begin{array}{l}\text { PCB } \\
\text { Piezotron- } \\
\text { ics }\end{array}$ & $\begin{array}{l}\mathrm{D}=9.5 \\
\mathrm{H}=21\end{array}$ & 8 & 10 \\
\hline 219A05 & 1-axis & $\begin{array}{l}\text { PCB } \\
\text { Piezotron- } \\
\text { ics }\end{array}$ & $\mathrm{D}=6, \mathrm{H}=6$ & 1.2 & 2500 \\
\hline FCM & 1 -axis & Sika & $\mathrm{D}=35, \mathrm{H}=15$ & 13 & up to 500 \\
\hline LBO/ LBC Series & 1-axis & $\begin{array}{l}\text { Transducer } \\
\text { Techniques }\end{array}$ & $\mathrm{D}=25, \mathrm{H}=10$ & - & - \\
\hline B105 & 1 -axis & $\begin{array}{l}\text { Brans Sen- } \\
\text { sor }\end{array}$ & $\mathrm{D}=8, \mathrm{H}=6$ & $\mathrm{n} / \mathrm{a}$ & $20-500$ \\
\hline MBA-TW Button & 1-axis & Flintec & $\begin{array}{l}\mathrm{D}=9.53, \quad \mathrm{H}- \\
6.35\end{array}$ & 8.5 & $20000 \%$ \\
\hline ISA Miniature S-Beam & 1 -axis & Flintec & $\begin{array}{l}\mathrm{L}=19, \\
\mathrm{~W}=16, \\
\mathrm{H}=4.7\end{array}$ & 9 & 200 \\
\hline FC20A & 1 -axis & Forsentek & $\mathrm{D}=20, \mathrm{H}=5$ & $\mathrm{n} / \mathrm{a}$ & 200 \\
\hline
\end{tabular}




\begin{tabular}{|c|c|c|c|c|c|}
\hline LLB130 & 1-axis & Futek & $\mathrm{D}=8, \mathrm{H}=3.3$ & & up to 250 \\
\hline Model 13 & 1-axis & Honeywell & $\begin{array}{l}\mathrm{D}=9.65, \\
\mathrm{H}=3.3\end{array}$ & 9 & up to 250 \\
\hline 9132B & 1-axis & Kistler & $\begin{array}{l}\mathrm{OD}=8, \\
\mathrm{ID}=2.7, \\
\mathrm{H}=3\end{array}$ & 1 & 3000 \\
\hline VLC856 & 1 -axis & $\begin{array}{l}\text { LORD } \\
\text { Sensing }\end{array}$ & $\begin{array}{l}\mathrm{D}=9.7 \\
\mathrm{H}=3.3\end{array}$ & $\mathrm{n} / \mathrm{a}$ & 200 \\
\hline KM10 & 1 -axis & $\begin{array}{l}\text { Me- Mebeta } \\
\text { System }\end{array}$ & $\mathrm{D}=9.8, \mathrm{H}=4$ & 10 & 200 \\
\hline LC302/LCM302 Series & 1-axis & Omega & $\begin{array}{l}\mathrm{D}=19, \\
\mathrm{H}=12.7\end{array}$ & $\mathrm{n} / \mathrm{a}$ & 200 \\
\hline LC307/LCM307 Series & 1-axis & Omega & $\begin{array}{l}\mathrm{D}=12.7, \\
\mathrm{H}=9.525\end{array}$ & $\mathrm{n} / \mathrm{a}$ & up to 1000 \\
\hline $\mathrm{S} 401$ & 1-axis & $\begin{array}{l}\text { Strain Mea- } \\
\text { surement } \\
\text { Devices }\end{array}$ & $\mathrm{D}=15, \mathrm{H}=8$ & $\mathrm{n} / \mathrm{a}$ & 200 \\
\hline $\mathrm{S} 400$ & 1-axis & $\begin{array}{l}\text { Strain Mea- } \\
\text { surement } \\
\text { Devices }\end{array}$ & $\begin{array}{l}\mathrm{D}=12.7, \\
\mathrm{H}=9.525\end{array}$ & $\mathrm{n} / \mathrm{a}$ & 200 \\
\hline AnyLoad 247AA & 1-axis & $\begin{array}{l}\text { Tacuna } \\
\text { Systems }\end{array}$ & $\begin{array}{l}\mathrm{D}=\quad 20, \\
\mathrm{H}=11\end{array}$ & $\begin{array}{l}9, \text { without } \\
\text { cable }\end{array}$ & 200 \\
\hline
\end{tabular}




\begin{tabular}{|c|c|c|c|c|c|}
\hline AnyLoad 247AS & 1 -axis & $\begin{array}{l}\text { Tacuna } \\
\text { Systems }\end{array}$ & $\begin{array}{l}\mathrm{D}=20 \\
\mathrm{H}=11\end{array}$ & $\begin{array}{l}9, \text { without } \\
\text { cable }\end{array}$ & 200 \\
\hline XFL212R & 1 -axis & $\begin{array}{l}\text { TE Connec- } \\
\text { tivity }\end{array}$ & $\begin{array}{l}\mathrm{D}=12.5 \\
\mathrm{H}=3.5\end{array}$ & $\mathrm{n} / \mathrm{a}$ & 200 \\
\hline $\mathrm{F} 1222$ & 1 -axis & Tecsis & $\begin{array}{l}\mathrm{D}=\quad 9.7, \\
\mathrm{H}=3.3\end{array}$ & $\mathrm{n} / \mathrm{a}$ & 200 \\
\hline SLB Series & 1 -axis & $\begin{array}{l}\text { Transducer } \\
\text { Techniques }\end{array}$ & $\begin{array}{l}\mathrm{D}=9.5 \\
\mathrm{H}=6.4\end{array}$ & $\mathrm{n} / \mathrm{a}$ & 200 \\
\hline DynPick & 3 -axis & $\begin{array}{l}\text { DynPick } \\
\text { (Wacoh- } \\
\text { Tech) }\end{array}$ & $\mathrm{D}=10, \mathrm{H}=7$ & $\mathrm{n} / \mathrm{a}$ & 10 \\
\hline USL08-H6 Series & 3 -axis & $\begin{array}{l}\text { Tec Gihan } \\
\text { Co., Ltd. }\end{array}$ & $\begin{array}{l}\mathrm{L}=28, \\
\mathrm{~W}=28, \mathrm{H}=6\end{array}$ & - & - \\
\hline USL06-H5 Series & 3 -axis & $\begin{array}{l}\text { Tec Gihan } \\
\text { Co., Ltd. }\end{array}$ & $\begin{array}{l}\mathrm{L}=20, \\
\mathrm{~W}=20, \\
\mathrm{H}=5\end{array}$ & $\begin{array}{l}7 \text {, without } \\
\text { cable }\end{array}$ & 200 \\
\hline USLO6-AP Series & 3 -axis & $\begin{array}{l}\text { Tec Gihan } \\
\text { Co., Ltd. }\end{array}$ & $\begin{array}{l}\mathrm{L}=20, \\
\mathrm{~W}=16, \\
\mathrm{H}=12\end{array}$ & $\mathrm{n} / \mathrm{a}$ & 200 \\
\hline Nano17 & 6 -axis & $\begin{array}{l}\text { ATI In- } \\
\text { dustrial } \\
\text { Automa- } \\
\text { tion }\end{array}$ & $\begin{array}{l}\mathrm{D}=17, \\
\mathrm{H}=14.5, \text { plus } \\
\text { wire exit }\end{array}$ & 9 & 70 \\
\hline
\end{tabular}




\begin{tabular}{|l|l|l|l|l|l|}
\hline K6D27 & 6 -axis & $\begin{array}{l}\text { Me- Mebeta } \\
\text { System }\end{array}$ & $\mathrm{D}=27, \mathrm{H}=25$ & 25 & 200 \\
\hline $3701 \mathrm{~A}$ & 6 -axis & $\begin{array}{l}\text { SRI Sunrise } \\
\text { Instru- } \\
\text { ments }\end{array}$ & $\begin{array}{l}\mathrm{OD}=15, \\
\mathrm{ID}=4.4, \\
\mathrm{H}=14\end{array}$ & 10 & 100 \\
\hline
\end{tabular}




\section{Appendix C \\ Touch Variation Study Ethics Clearance and Questionnaire}


Office of Research Ethics

5110 Human Computer Interaction Bldg | 1125 Colonel By Drive

I Ottawa, Ontario K1S 5B6

613-520-2600 Ext: 4085

ethics@carleton.ca

\section{CERTIFICATION OF INSTITUTIONAL ETHICS CLEARANCE}

The Carleton University Research Ethics Board-B (CUREB-B) has granted ethics clearance for the research project described below and research may now proceed. CUREB-B is constituted and operates in compliance with the Tri-Council Policy Statement: Ethical Conduct for Research Involving Humans (TCPS2).

Ethics Protocol Clearance ID: Project \# 109919

Research Team: Ms. Francesca Tsimiklis (Primary Investigator)

Dr. Donald L. Russell (Research Supervisor)

Project Title: Sensitivity to Touch Variations in Piano Keys [Francesca Tsimiklis]

Funding Source (If applicable):

Effective: November 22, 2018

Expires: November 30, 2019.

Please ensure the study clearance number is prominently placed in all recruitment and consent materials: CUREB-B Clearance \# 109919.

\section{Restrictions:}

This certification is subject to the following conditions:

1. Clearance is granted only for the research and purposes described in the application.

2. Any modification to the approved research must be submitted to CUREB-B via a Change to Protocol Form. All changes must be cleared prior to the continuance of the research.

3. An Annual Status Report for the renewal of ethics clearance must be submitted and cleared by the renewal date listed above. Failure to submit the Annual Status Report will result in the closure of the file.If funding is associated, funds will be frozen.

4. A closure request must be sent to CUREB-B when the research is complete or terminated.

5. During the course of the study, if you encounter an adverse event, material incidental finding, protocol deviation or other unanticipated problem, you must complete and submit a Report of Adverse Events and Unanticipated Problems Form, found here: https://carleton.ca/researchethics/forms-and-templates/ 
Failure to conduct the research in accordance with the principles of the Tri-Council Policy Statement: Ethical Conduct for Research Involving Humans 2ndedition and the Carleton University Policies and Procedures for the Ethical Conduct of Research may result in the suspension or termination of the research project.

Upon reasonable request, it is the policy of CUREB, for cleared protocols, to release the name of the PI, the title of the project, and the date of clearance and any renewal(s).

Please contact the Research Compliance Coordinators, at ethics@carleton.ca, if you have any questions.

CLEARED BY: Date: November 22, 2018

Bernadette Campbell, PhD, Chair, CUREB-B

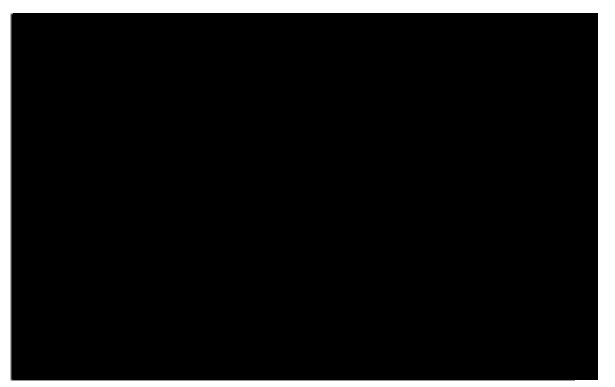

Natasha Artemeva, PhD, Vice-Chair, CUREB-B 


\section{Carleton \\ U N I V E R S I T Y}

Office of Research Ethics

503 Robertson Hall | 1125 Colonel By Drive

Ottawa, Ontario K1S 5B6

613-520-2600 Ext: 4085

ethics@carleton.ca

\section{CERTIFICATION OF INSTITUTIONAL ETHICS CLEARANCE}

The Carleton University Research Ethics Board-B (CUREB-B) at Carleton University has renewed ethics clearance for the research project detailed below. CUREB-B is constituted and operates in compliance with the Tri-Council Policy Statement: Ethical Conduct for Research Involving Humans (TCPS2).

Title: Sensitivity to Touch Variations in Piano Keys [Francesca Tsimiklis]

Protocol \#: 109919

Principal Investigator: Ms. Francesca Tsimiklis

Department and Institution: Faculty of Engineering and Design\Mechanical \& Aerospace Engineering (Department of),

Project Team (and Roles): Ms. Francesca Tsimiklis (Primary Investigator)

Dr. Donald L. Russell (Research Supervisor)

Funding Source (If applicable):

Effective: December 03, 2019

Expires: December 31, 2020.

Please ensure the study clearance number is prominently placed in all recruitment and consent materials: CUREB-B Clearance \# 109919.

\section{Restrictions:}

This certification is subject to the following conditions:

1. Clearance is granted only for the research and purposes described in the application.

2. Any modification to the approved research must be submitted to CUREB-B. All changes must be approved prior to the continuance of the research. 
3. An Annual Application for the renewal of ethics clearance must be submitted and cleared by the above date. Failure to submit the Annual Status Report will result in the closure of the file. If funding is associated, funds will be frozen.

4. A closure request must be sent to CUREB-B when the research is complete or terminated.

5.During the course of the study, if you encounter an adverse event, material incidental finding, protocol deviation or other unanticipated problem, you must complete and submit a Report of Adverse Events and Unanticipated Problems Form, found

here:https://carleton.ca/researchethics/forms-and-templates/

6. It is the responsibility of the student to notify their supervisor of any adverse events, changes to their application, or requests to renew/close the protocol.

7. Failure to conduct the research in accordance with the principles of the Tri-Council Policy Statement: Ethical Conduct for Research Involving Humans 2nd edition and the Carleton University Policies and Procedures for the Ethical Conduct of Research may result in the suspension or termination of the research project.

Upon reasonable request, it is the policy of CUREB, for cleared protocols, to release the name of the PI, the title of the project, and the date of clearance and any renewal(s).

Please email the Research Compliance Coordinators at ethics@carleton.ca if you have any questions.

\section{CLEARED BY:}

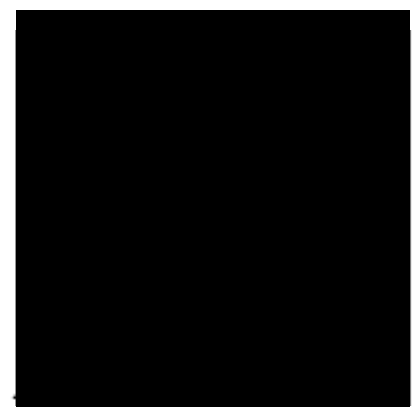

Natasha Artemeva, PhD, Chair, CUREB-B

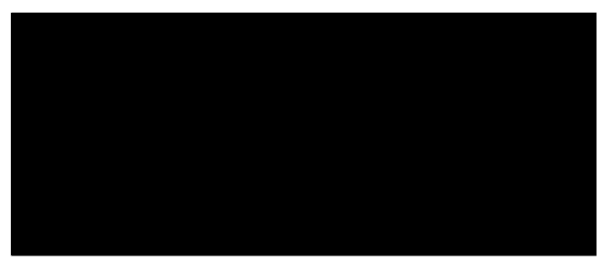

Janet Mantler, PhD, Vice-Chair, CUREB-B
Date: December 03, 2019 


\section{Sensitivity to Key Touch Variations in Piano Keys Participant Questionnaire Form}

\section{Participant Code:}

Please do not remove the paper covers from the keyboard at any time. The testing will be concerned with the covered white keys.

After testing, please do not discuss your findings with others, as this could alter other participants test results.

Please complete the steps below and answer the corresponding questions.

\section{Test 1:}

1) Position yourself at the piano to complete an ascending and descending $\mathrm{C}$ Major 3-octave scale with your right hand, starting at the indicated $\mathrm{C}$ in the covered test range.

2) Complete the $\mathrm{C}$ Major scale three (3) times at a steady tempo (approximately $d=88$ ). A metronome will provide the starting tempo.

3) Read and answer the following questions and write your answer directly on this questionnaire form.

Did any key(s) feel different during the scale exercise? If so, please put an ' $\mathrm{X}$ ' on those key(s) on the key range shown below.

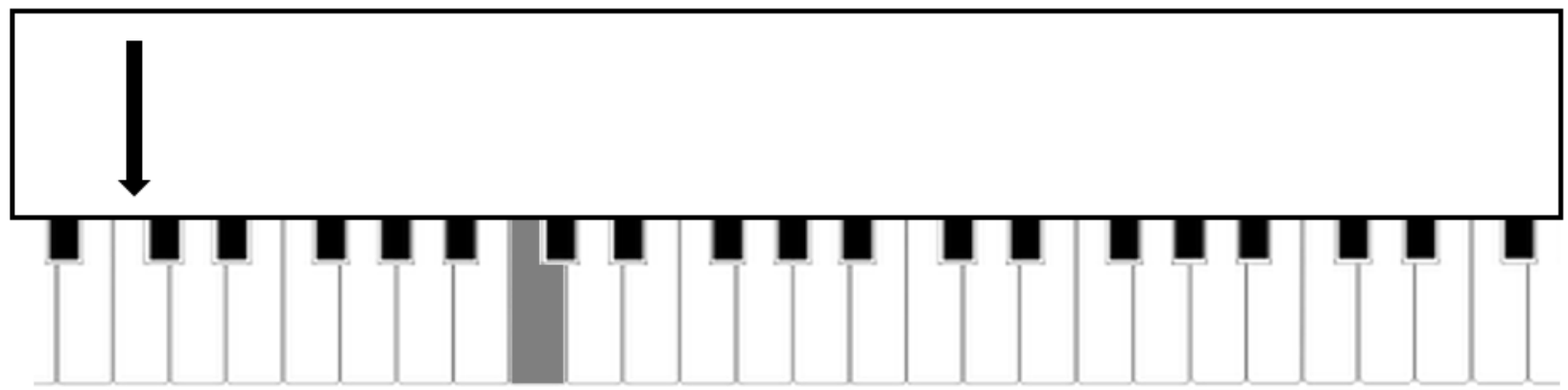

If any key(s) were indicated above, how did they feel different? Consider the touch and response of the keys. 
4) Position yourself at the piano again. Play any keys or patterns within the white keys of the test range to further investigate the keys to determine which, if any, keys are different (ex. playing the one key with a repetitive touch, playing with a faster or slower tempo).

5) Read and answer the following questions and write your answer directly on this questionnaire form.

Did you notice any additional details about the keys during the investigative playing exercise? If so, please put an 'X' on those key(s) on key range shown below.

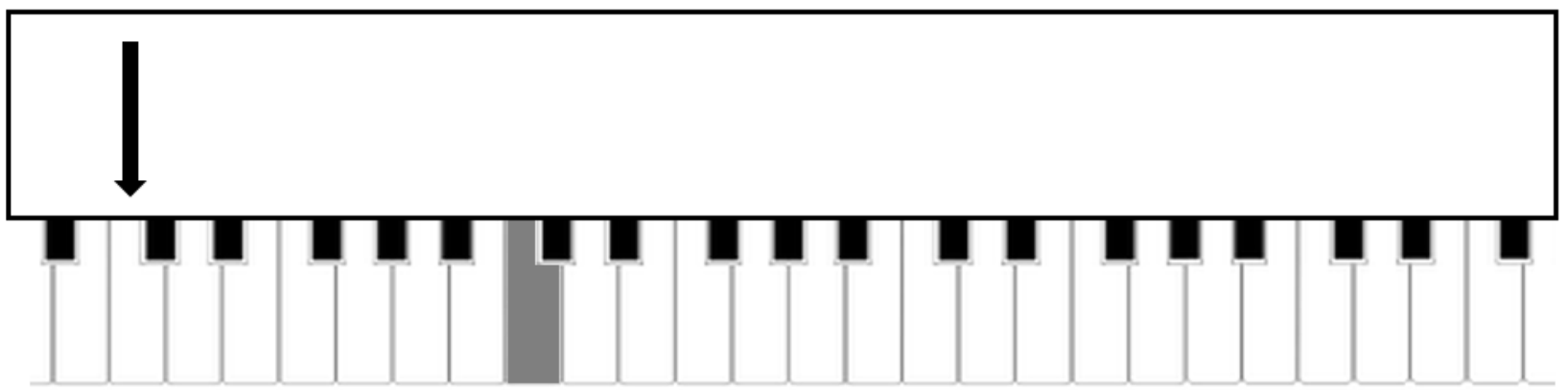

If any key(s) were indicated above, how did they feel different? Consider the touch and response of the keys.

If any key(s) were indicated above, please describe how you determined the key(s) to be different in the test range (ex. playing the one key with a repetitive touch, playing with a faster or slower tempo). 
6) Please complete the next question at the desk provided by the research team. During this time the research team may adjust the key touch for the second set of testing.

Given the two playing test opportunities to investigate the keys in the test range, please draw conclusions on which key(s) you believe have been altered, if any. Please put an ' $\mathrm{X}$ ' on the key(s), if any, on key range shown below that you believe are different. If you have an idea on how they may be different, please list that as well.

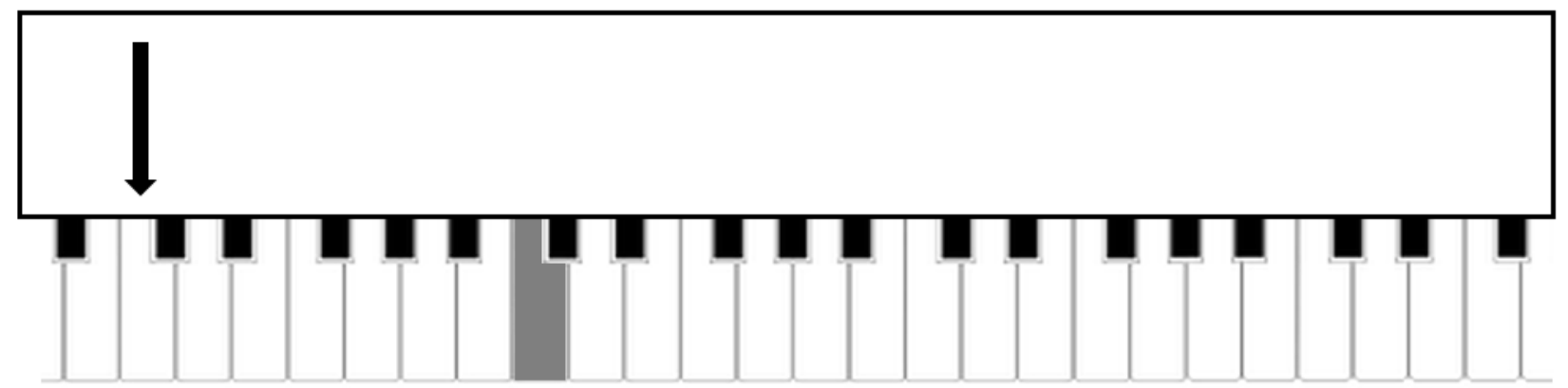




\section{Test 2:}

1) Position yourself at the piano to complete an ascending and descending $\mathrm{C}$ Major 3-octave scale with your right hand, starting at the indicated $\mathrm{C}$ in the covered test range.

2) Complete the $\mathrm{C}$ Major scale three (3) times at a steady tempo (approximately $d=88$ ). A metronome will provide the starting tempo.

3) Read and answer the following questions and write your answer directly on this questionnaire form.

Did any key(s) feel different during the scale exercise? If so, please put an ' $\mathrm{X}$ ' on those key(s) on the key range shown below.

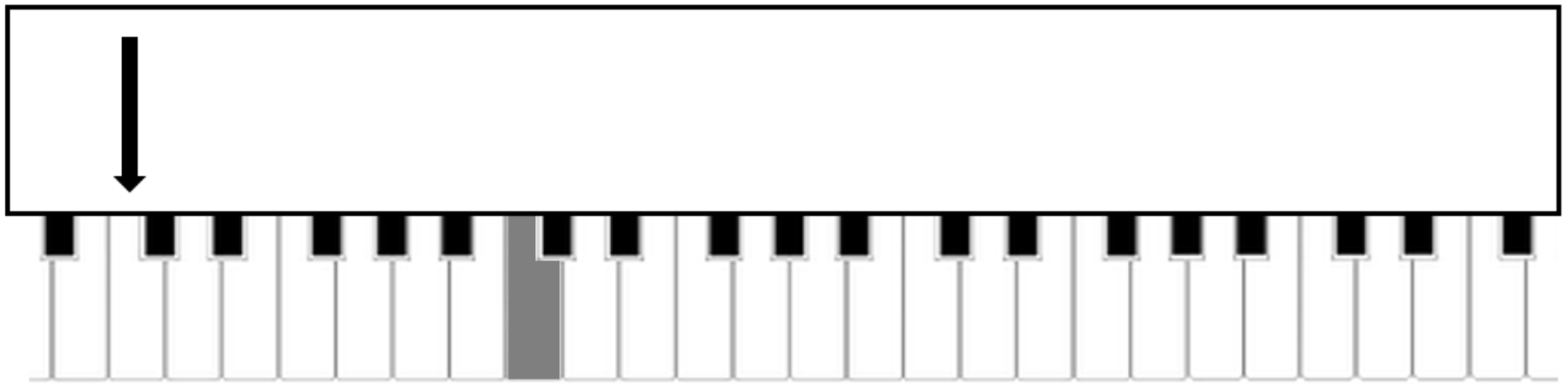

If any key(s) were indicated above, how did they feel different? Consider the touch and response of the keys. 
4) Position yourself at the piano again. Play any keys or patterns within the white keys of the test range to further investigate the keys to determine which, if any, keys are different (ex. playing the one key with a repetitive touch, playing with a faster or slower tempo).

5) Read and answer the following questions and write your answer directly on this questionnaire form.

Did you notice any additional details about the keys during the investigative playing exercise? If so, please put an 'X' on those key(s) on key range shown below.

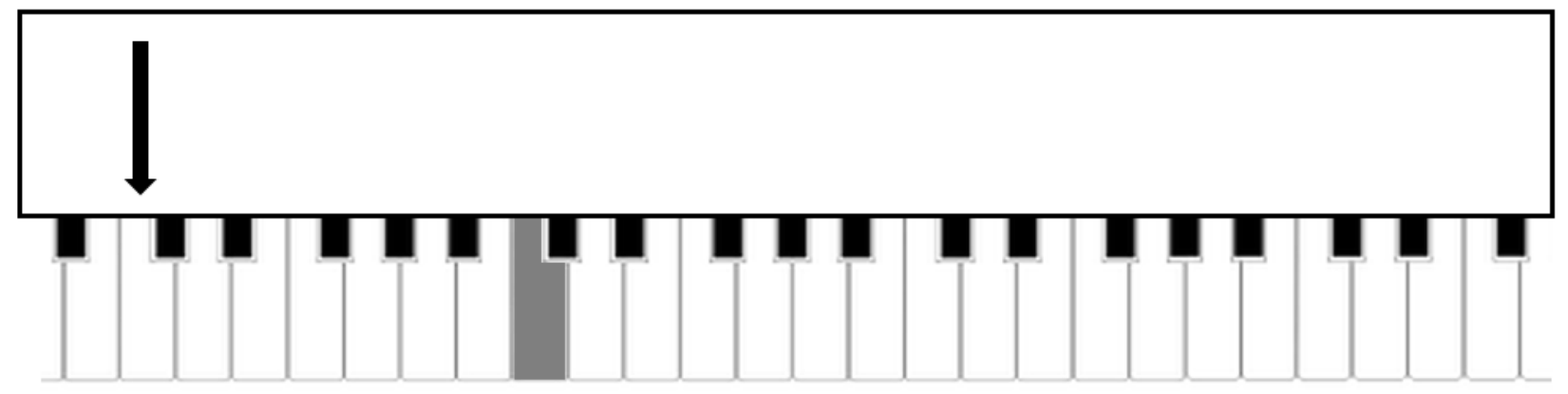

If any key(s) were indicated above, how did they feel different? Consider the touch and response of the keys.

If any key(s) were indicated above, please describe how you determined the key(s) to be different in the test range (ex. playing the one key with a repetitive touch, playing with a faster or slower tempo). 
6) Please complete the next question at the desk provided by the research team. During this time the research team may adjust the key touch for the second set of testing.

Given the two playing test opportunities to investigate the keys in the test range, please draw conclusions on which key(s) you believe have been altered, if any. Please put an ' $\mathrm{X}$ ' on the key(s), if any, on key range shown below that you believe are different. If you have an idea on how they may be different, please list that as well.

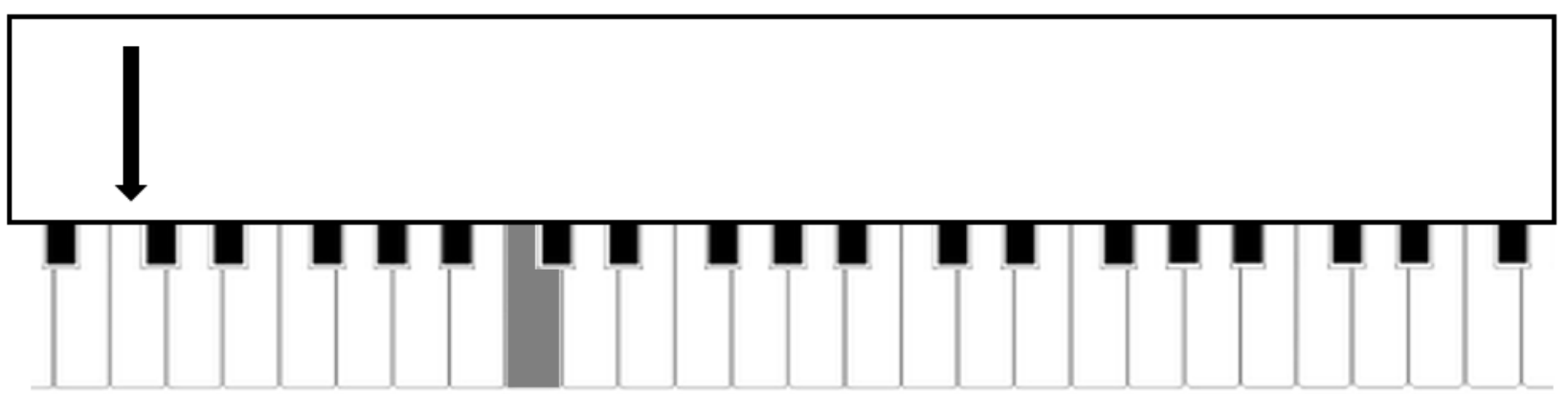




\section{Appendix D}

\section{Code Listing: Verification of Theoretical}

\section{Analysis Script}

This appendix contains the code used to verify the theoretical analysis which was completed in the design and analysis stages of the prototype.

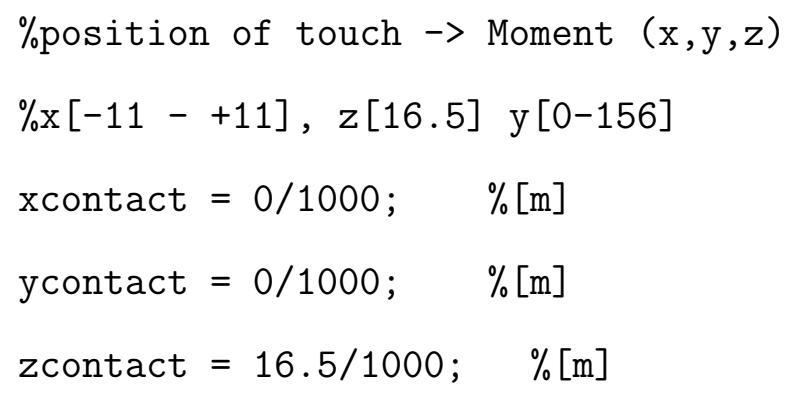


Vout_r12_45_yA, Vout_r12_90_yA, Vout_r23_0_yA, Vout_r23_45_yA, ... Vout_r23_90_yA, Vout_r12_0_yB, Vout_r12_45_yB, Vout_r12_90_yB, ... Vout_r23_0_yB, Vout_r23_45_yB, Vout_r23_90_yB, stress_yA, stress_yB ... ] = sg_assessment $(\mathrm{Fx}, \mathrm{Fy}, \mathrm{Fz}, \mathrm{xcontact}, \mathrm{ycontact}, \mathrm{zcontact})$;

$\%$ store subfunction outputs $\operatorname{outputs}(i,:)=\left[F x, F y, F z, x c o n t a c t, y c o n t a c t, z c o n t a c t, V o u t \_r 12 \_0 \_y A, \ldots\right.$ Vout_r12_45_yA, Vout_r12_90_yA, Vout_r23_0_yA, Vout_r23_45_yA,... Vout_r23_90_yA, Vout_r12_0_yB, Vout_r12_45_yB, Vout_r12_90_yB,... Vout_r23_0_yB, Vout_r23_45_yB, Vout_r23_90_yB] ; microstrains $(i,:)=\{$ microstrain0_yA, microstrain45_yA, microstrain90_yA,... microstrain0_yB, microstrain45_yB, microstrain90_yB\}; stressoutput $(i,:)=\{$ stress_yA, stress_yB $\}$ $i=i+1$;

$\%$ end end

\%extract the microstrains

microR1 $=\operatorname{cellfun}(@(v) v(1)$, microstrains $)$; microR2 = cellfun (@(v)v(2), microstrains); microR3 $=\operatorname{cellfun}(@(\mathrm{v}) \mathrm{v}(3)$, microstrains $)$; microR4 $=\operatorname{cellfun}(@(\mathrm{v}) \mathrm{v}(4)$, microstrains $)$;

\%graph the outputs plotgraphs (microR1, microR2, microR3, microR4, outputs); 
$\%$ save the data for future review

filename1 = 'rosetteanalysis_outputs2.csv';

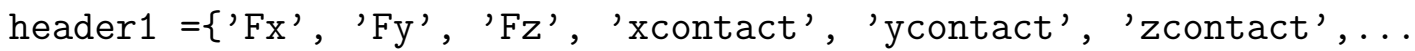

'Vout_r12_0_yA', 'Vout_r12_45_yA' , 'Vout_r12_90_yA' , 'Vout_r23_0_yA' , . . .

'Vout_r23_45_yA', 'Vout_r23_90_yA', 'Vout_r12_0_yB' , 'Vout_r12_45_yB' , . .

'Vout_r12_90_yB', 'Vout_r23_0_yB' , 'Vout_r23_45_yB' , 'Vout_r23_90_yB'\};

csvwrite(filename1, outputs);

\section{$\% \% \% \% \% \% \% \% \% \% \% \% \% \% \% \% \% \% \% \% \% \% \% \% \% \% \% \% \% \% \% \% \% \%$}

$\%$ functions used:

function [] =plotgraphs (microR1, microR2, microR3, microR4, outputs)

$\% \mathrm{Pl}$ ane A

figure (1)

subplot $(3,1,1)$

$\%$ plot (outputs $\left.(:, 1), \operatorname{microR} 1(:, 1), \mathrm{k}^{\prime}\right) \% \mathrm{x}$

$\%$ hold on

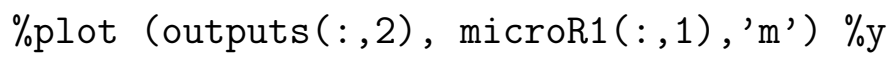

plot (outputs $(:, 3)$, microR1 $\left.(:, 1),{ }^{\prime} b^{\prime}\right) \% z$

$\%$ hold off

title('Microstrain Output for Fz Force Variation at Position A1 0')

xlabel ('Force Applied (N)')

ylabel ('Microstrain Output')

\%legend( 'Fx', 'Fy', 'Fz')

subplot $(3,1,2)$

\%plot (outputs $(:, 1)$, microR1 $\left.(:, 2),{ }^{\prime} \mathrm{k}^{\prime}\right)$

$\%$ hold on

\%plot (outputs $\left.(:, 2), \operatorname{microR} 1(:, 2), \mathrm{Im}^{\prime}\right)$ 
plot (outputs $\left.(:, 3), \operatorname{microR} 1(:, 2), \mathrm{b}^{\prime}\right)$

$\%$ hold off

title ('Microstrain Output for Fz Force Variation at Position A1 45')

xlabel ('Force Applied (N)')

ylabel ('Microstrain Output')

subplot $(3,1,3)$

\%plot (outputs $\left.(:, 1), \operatorname{microR} 1(:, 3), \mathrm{k}^{\prime}\right)$

$\%$ hold on

\%plot (outputs $\left.(:, 2), \operatorname{microR} 1(:, 3),{ }^{\prime} \mathrm{m}^{\prime}\right)$

plot (outputs $\left.(:, 3), \operatorname{microR} 1(:, 3), \mathrm{b}^{\prime}\right)$

$\%$ hold off

title ('Microstrain Output for Fz Force Variation at Position A1 90')

xlabel ('Force Applied (N)')

ylabel ('Microstrain Output')

figure (2)

subplot $(3,1,1)$

$\%$ plot (outputs $(:, 1)$, microR2 $\left.(:, 1),{ }^{\prime} k^{\prime}\right)$

$\%$ hold on

$\%$ plot (outputs $\left.(:, 2), \operatorname{microR} 2(:, 1), \mathrm{m}^{\prime}\right)$

plot (outputs $(:, 3), \operatorname{microR2}(:, 1),{ }^{\prime} \mathrm{b}$ ')

$\%$ hold off

title('Microstrain Output for Fz Force Variation at Position A2 0')

xlabel ('Force Applied (N)')

ylabel ('Microstrain Output')

$\%$ legend( 'Fx', 'Fy', 'Fz')

subplot $(3,1,2)$ 
\%plot (outputs $\left.(:, 1), \operatorname{microR} 2(:, 2), \mathrm{k}^{\prime}\right)$

$\%$ hold on

\%plot (outputs $(:, 2), \operatorname{microR} 2(:, 2)$, 'm')

plot (outputs $\left.(:, 3), \operatorname{microR2}(:, 2), \mathrm{b}^{\prime}\right)$

$\%$ hold off

title ('Microstrain Output for Fz Force Variation at Position A2 45')

xlabel ('Force Applied (N)')

ylabel ('Microstrain Output')

subplot $(3,1,3)$

\%plot (outputs $\left.(:, 1), \operatorname{microR} 2(:, 3), \mathrm{k}^{\prime}\right)$

$\%$ hold on

\%plot (outputs $\left.(:, 2), \operatorname{microR2}(:, 3), \mathrm{g}^{\prime}\right)$

plot (outputs $\left.(:, 3), \operatorname{microR} 2(:, 3), \mathrm{b}^{\prime}\right)$

$\%$ hold off

title ('Microstrain Output for Fz Force Variation at Position A2 90')

xlabel ('Force Applied (N)')

ylabel ('Microstrain Output')

figure (3)

subplot $(3,1,1)$

\%plot (outputs $\left.(:, 1), \operatorname{microR3}(:, 1), \mathrm{k}^{\prime}\right)$

$\%$ hold on

\%plot (outputs $\left.(:, 2), \operatorname{microR} 3(:, 1), \mathrm{g}^{\prime}\right)$

plot (outputs $\left.(:, 3), \operatorname{microR3}(:, 1), \mathrm{b}^{\prime}\right)$

$\%$ hold off

title('Microstrain Output for Fz Force Variation at Position A3 0')

xlabel ('Force Applied (N)') 
ylabel ('Microstrain Output')

\%legend ('Fx', 'Fy', 'Fz')

subplot $(3,1,2)$

\%plot (outputs $\left.(:, 1), \operatorname{microR3}(:, 2), \mathrm{k}^{\prime}\right)$

$\%$ hold on

\%plot (outputs $(:, 2), \operatorname{microR} 3(:, 2)$, 'm')

plot (outputs $\left.(:, 3), \operatorname{microR} 3(:, 2), \mathrm{b}^{\prime}\right)$

$\%$ hold off

title ('Microstrain Output for Fz Force Variation at Position A3 45')

xlabel ('Force Applied (N)')

ylabel ('Microstrain Output')

subplot $(3,1,3)$

\%plot (outputs $\left.(:, 1), \operatorname{microR} 3(:, 3), \mathrm{k}^{\prime}\right)$

$\%$ hold on

\%plot (outputs $(:, 2), \operatorname{microR3}(:, 3)$, 'm' $\left.^{\prime}\right)$

plot (outputs $\left.(:, 3), \operatorname{microR} 3(:, 3), \mathrm{b}^{\prime}\right)$

$\%$ hold off

title ('Microstrain Output for Fz Force Variation at Position A3 90')

xlabel ('Force Applied (N)')

ylabel ('Microstrain Output')

figure (4)

subplot $(3,1,1)$

\%plot (outputs $\left.(:, 1), \operatorname{microR} 4(:, 1), \mathrm{k}^{\prime}\right)$

$\%$ hold on

\%plot (outputs $(:, 2), \operatorname{microR} 4(:, 1)$, 'm')

plot (outputs $\left.(:, 3), \operatorname{microR} 4(:, 1),{ }^{\prime} b^{\prime}\right)$ 
$\%$ hold off

title('Microstrain Output for Fz Force Variation at Position A4 0')

xlabel ('Force Applied (N)')

ylabel ('Microstrain Output')

$\%$ legend ('Fx', 'Fy', 'Fz')

subplot $(3,1,2)$

\%plot (outputs $\left.(:, 1), \operatorname{microR} 4(:, 2), \mathrm{k}^{\prime}\right)$

$\%$ hold on

\%plot (outputs $(:, 2), \operatorname{microR} 4(:, 2)$, 'm')

plot (outputs $(:, 3), \operatorname{microR} 4(:, 2)$, 'b')

$\%$ hold off

title ('Microstrain Output for Fz Force Variation at Position A4 45')

xlabel ('Force Applied (N)')

ylabel ('Microstrain Output')

subplot $(3,1,3)$

\%plot (outputs $\left.(:, 1), \operatorname{microR} 4(:, 3), \mathrm{k}^{\prime}\right)$

$\%$ hold on

\%plot (outputs $\left.(:, 2), \operatorname{microR} 4(:, 3), \mathrm{g}^{\prime}\right)$

plot (outputs $\left.(:, 3), \operatorname{microR} 4(:, 3), \mathrm{b}^{\prime}\right)$

title ('Microstrain Output for Fz Force Variation at Position A4 90')

xlabel ('Force Applied (N)')

ylabel ('Microstrain Output')

$\%$ hold off

$\%$ Plane B

figure (5)

subplot $(3,1,1)$ 
\%plot (outputs $\left.(:, 1), \operatorname{microR} 1(:, 4), \mathrm{k}^{\prime}\right)$

$\%$ hold on

\%plot (outputs $(:, 2), \operatorname{microR} 1(:, 4)$, 'm')

plot (outputs $\left.(:, 3), \operatorname{microR} 1(:, 4), \mathrm{b}^{\prime}\right)$

$\%$ hold off

title('Microstrain Output for Fz Force Variation at Position B1 0')

xlabel ('Force Applied (N)')

ylabel ('Microstrain Output')

\%legend( 'Fx', 'Fy', 'Fz')

$\operatorname{subplot}(3,1,2)$

\%plot (outputs $\left.(:, 1), \operatorname{microR} 1(:, 5), \mathrm{k}^{\prime}\right)$

$\%$ hold on

\%plot (outputs $(:, 2)$, microR1 $(:, 5)$, 'm')

plot (outputs $(:, 3)$, microR1 $\left.(:, 5),{ }^{\prime} b^{\prime}\right)$

$\%$ hold off

title ('Microstrain Output for Fz Force Variation at Position B1 45')

xlabel ('Force Applied (N)')

ylabel ('Microstrain Output')

subplot $(3,1,3)$

$\%$ plot $\left(\operatorname{outputs}(:, 1), \operatorname{microR} 1(:, 6), \mathrm{k}^{\prime}\right)$

$\%$ hold on

\%plot (outputs $(:, 2), \operatorname{microR} 1(:, 6)$, 'm')

plot (outputs $\left.(:, 3), \operatorname{microR} 1(:, 6), \mathrm{b}^{\prime}\right)$

$\%$ hold off

title ('Microstrain Output for Fz Force Variation at Position B1 90')

xlabel ('Force Applied (N)')

ylabel ('Microstrain Output') 


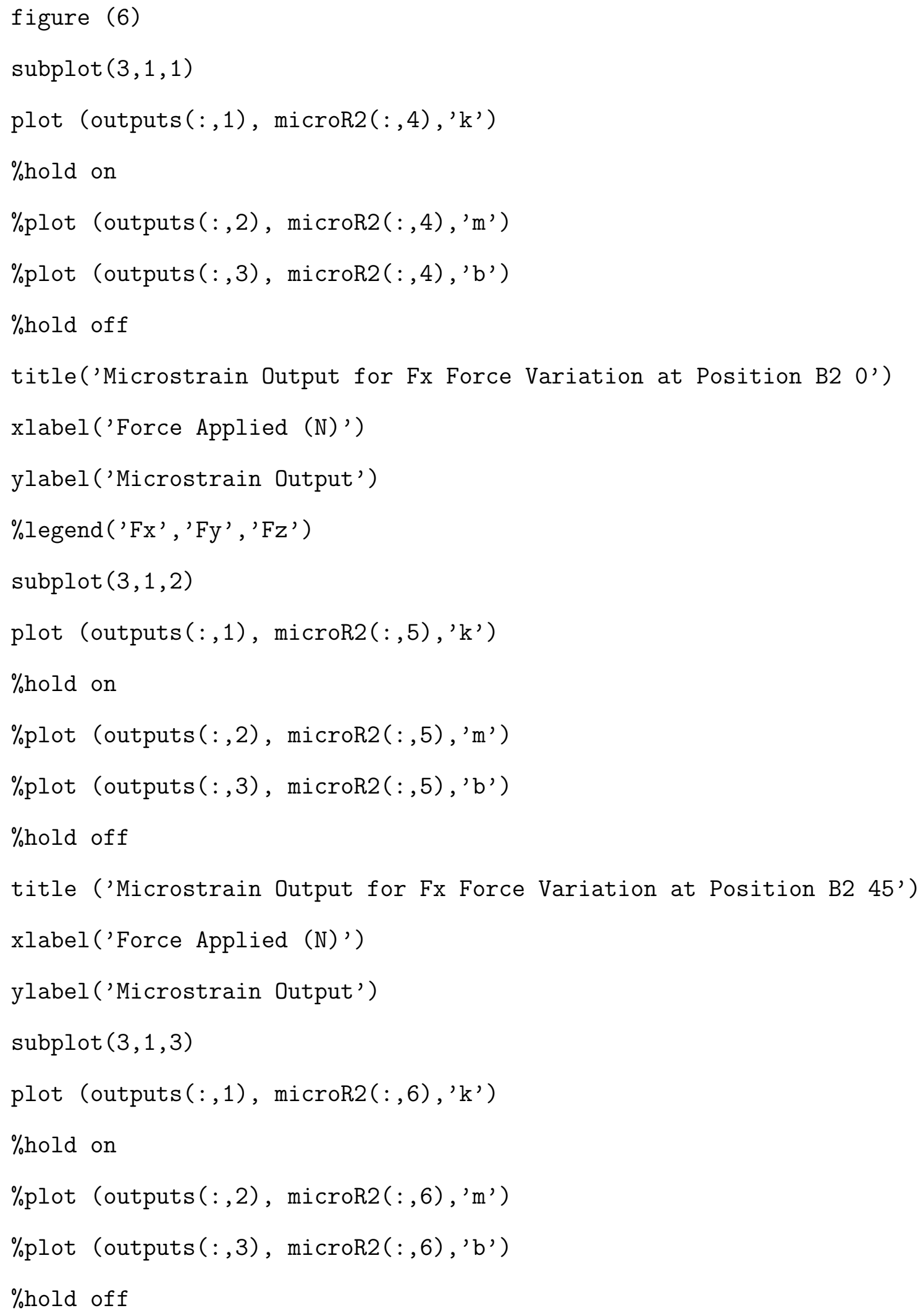


title ('Microstrain Output for Fx Force Variation at Position B2 90') xlabel ('Force Applied (N)')

ylabel ('Microstrain Output')

figure (7)

subplot $(3,1,1)$

plot (outputs $\left.(:, 2), \operatorname{microR} 3(:, 4),{ }^{\prime} \mathrm{k}^{\prime}\right)$

$\%$ hold on

\%plot (outputs $(:, 2)$, microR3 $(:, 4)$, 'm')

\%plot (outputs $(:, 3), \operatorname{microR} 3(:, 4)$, 'b')

$\%$ hold off

title('Microstrain Output for Fx Force Variation at Position B3 0')

xlabel ('Force Applied (N)')

ylabel ('Microstrain Output')

$\%$ legend( 'Fx', 'Fy', 'Fz')

$\operatorname{subplot}(3,1,2)$

plot (outputs $\left.(:, 1), \operatorname{microR3}(:, 5),{ }^{\prime} \mathrm{k}^{\prime}\right)$

$\%$ hold on

\%plot (outputs $(:, 2), \operatorname{microR} 3(:, 5)$, 'm')

\%plot (outputs $(:, 3), \operatorname{microR3}(:, 5)$, 'b')

$\%$ hold off

title ('Microstrain Output for Fx Force Variation at Position B3 45')

xlabel ('Force Applied (N)')

ylabel ('Microstrain Output')

subplot $(3,1,3)$

plot (outputs $\left.(:, 1), \operatorname{microR} 3(:, 6),{ }^{\prime} \mathrm{k}^{\prime}\right)$

$\%$ hold on 


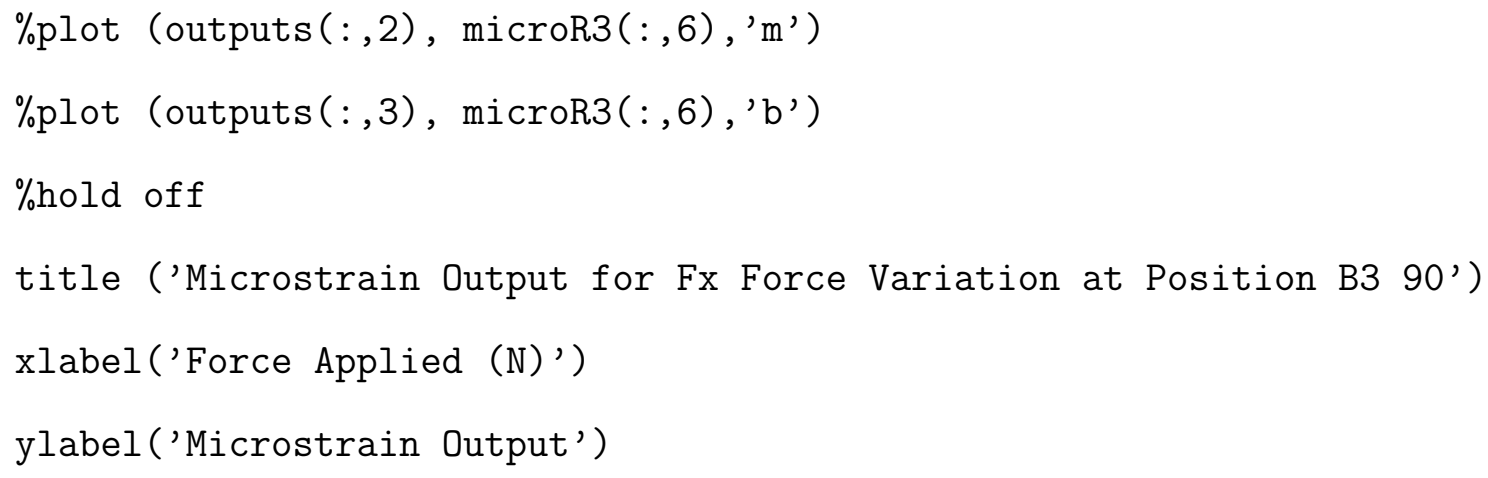




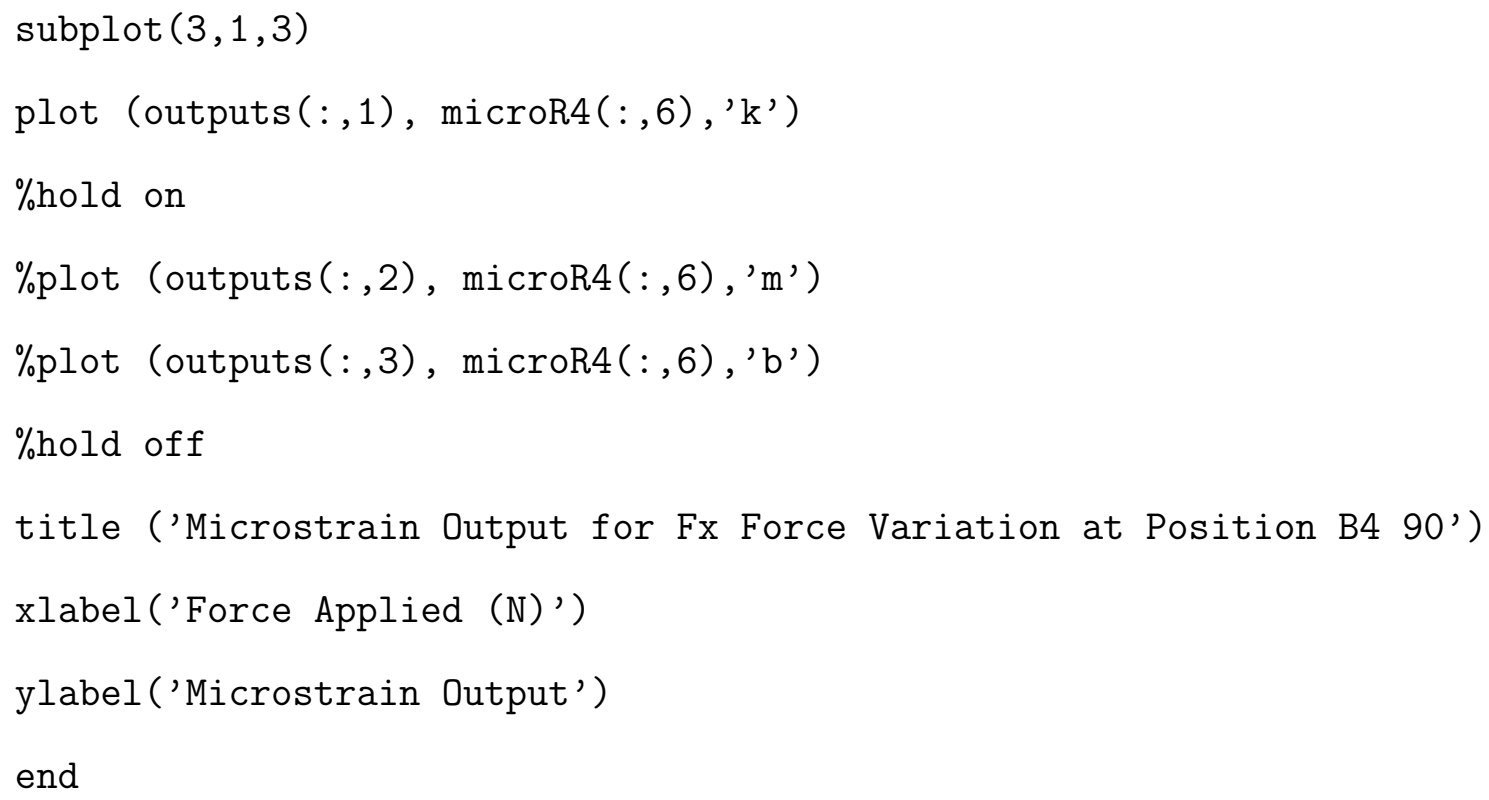


$\mathrm{v}=0.32$

$\%$ geometry of sensing beam

$\%$ comparision of different cross-sectional areas can be compared by changing the geoemetry variables

hbeam2 $=7 / 1000 ; \%[\mathrm{~m}]$

hbeam $=$ hbeam $2 / 2$;

wbeam2 $=7 / 1000 ; \%[\mathrm{~m}]$

wbeam $=$ wbeam $2 / 2$;

$t=0 / 1000 ;[\mathrm{m}] \%$ use if applying a hollow cross-section

lbeam $=95 / 1000 ; \%[\mathrm{~m}]$

$\% \%$ Reference [Appendix B-1a, pg. 813, Fundamentals of Machine Component Design, 5th ed., Juvinall and Marshek]

$I_{-} z z_{-}$out $=(($hbeam 2$) *($ wbeam 2$) \wedge 3) ; \%\left[m^{\wedge} 4\right]$

$\% I_{-} z z_{-}$in $=(($hbeam $2-(2 * t)) *(($ wbeam $2-(2 * t)) \wedge 3)) ; \%\left[m^{\wedge} 4\right]$

\%use for a hollow cross-section

$I_{-} z z_{-}$in $=0$;

Ibeam_zz $=(1 / 12) *\left(I_{\_} z z_{-}\right.$out $-I_{\_} z z_{-}$in $) ; \%\left[m^{\wedge} 4\right]$

I_xx_out $=(($ wbeam 2$) *($ hbeam 2$) \wedge 3)$;

$\% I_{-} x_{-}$in $=(($wbeam $2-(2 * t)) *(($ hbeam $2-(2 * t)) \wedge 3))$;

\%use for a hollow cross-section

$I_{-} x_{-}$in $=0$;

Ibeam_xx $=(1 / 12) *\left(I_{-} x_{-}\right.$out $-I_{-} x_{-}$in $) ; \%\left[m^{\wedge} 4\right]$

a_zy = lbeam;

b_zy = hbeam 2 ;

a_xy $=$ wbeam2; 
b_xy = lbeam;

Area_zy $=$ wbeam $2 *$ hbeam 2 ;

Area_xy $=$ wbeam $2 * 1$ beam;

$\% \%$ Reference [Eq'n 4.5, pg. 136, Fundamentals of Machine Component Design, 5th ed., Juvinall and Marshek]

TorsionCoeff_zy $=\left(\left(\left(3 * a_{-} z y\right)+\left(1.8 * b_{-} \_y\right)\right) /\left(\left(a_{-} z y^{\wedge} 2\right) *\left(b_{-} z y^{\wedge} 2\right)\right)\right)$;

TorsionCoeff_xy $=\left(\left(\left(3 * a_{-} x_{y}\right)+\left(1.8 * b_{-} \_x y\right)\right) /\left(\left(a_{-} y^{\wedge} 2\right) *\left(b_{-} y^{\wedge} 2\right)\right)\right)$;

\%Properties of the Strain Gauge Rosettes

$\mathrm{GF}=2 ; \quad \%$ gauge factor

Rnom $=120 ; \%$ nominal resistance $[\mathrm{ohm}]$

Vex $=3 ; \quad \%$ excitation voltage [volts]

Rref $=120 ; \%$ resistance of high-precision reference resistors for bridge [ohm]

\%geometry of key

y_B $=(60 / 1000)+$ hbeam $2 ; \%[\mathrm{~m}]$

$\mathrm{y}_{-} \mathrm{A}=\mathrm{y}_{-} \mathrm{B}+$ lbeam $-(2 *$ hbeam 2$) ; \quad \%[\mathrm{~m}]$

$\%$ with the defined variables, moment expressions can be developed

Mx_o = Fz*ycontact - Fy*zcontact;

My_o = Fx*zcontact - Fz*xcontact;

Mz_o $=$ Fy*xcontact - Fx*ycontact; 
$\% \% \% \%$ Stress Matrices Setup $\% \% \% \%$

$\%$ set up matrices as developed in theoretical analysis $\% \%$ y_A $\% \%$

c_12 $=\left(\left(-\left(\right.\right.\right.$ hbeam^$\left.\left.^{\wedge} 2\right)\right) /$ Ibeam_xx $)-(1 /($ wbeam $2 *$ hbeam 2$)) ;$

c_13 = (hbeam*y_A) /Ibeam_xx;

c_14 = (-hbeam) / Ibeam_xx;

c_31 $=((3 / 2) *(1 /$ wbeam $2 *$ lbeam $))+($ hbeam*TorsionCoeff_xy $)$;

c_35 = -TorsionCoeff_xy;

$c_{-} 42=\left(\left(-\left(\right.\right.\right.$ hbeam² $\left.\left.^{-2}\right)\right) /$ Ibeam_xx $)-(1 /($ wbeam $2 *$ hbeam 2$)) ;$

C_43 $=($-hbeam*y_A $) /$ Ibeam_xx;

C_44 = (hbeam) /Ibeam_xx;

c_61 $=((3 / 2) *(1 /$ wbeam $2 *$ lbeam $))+(-$ hbeam*TorsionCoeff_xy $)$;

c_65 = -TorsionCoeff_xy;

C_71 = (wbeam*y_A) /Ibeam_zz;

c_72 $=\left(\left(\right.\right.$ wbeam^2) ${ }^{\wedge} /$ Ibeam_zz $)-(1 /($ wbeam $2 *$ hbeam 2$)) ;$

c_76 = (wbeam/Ibeam_zz);

c_91 = (-y_A*TorsionCoeff_zy);

c_92 $=((3 /(2 *(1$ beam*hbeam 2$)))-($ wbeam*TorsionCoeff_zy $)) ;$

c_96 = -TorsionCoeff_zy; 


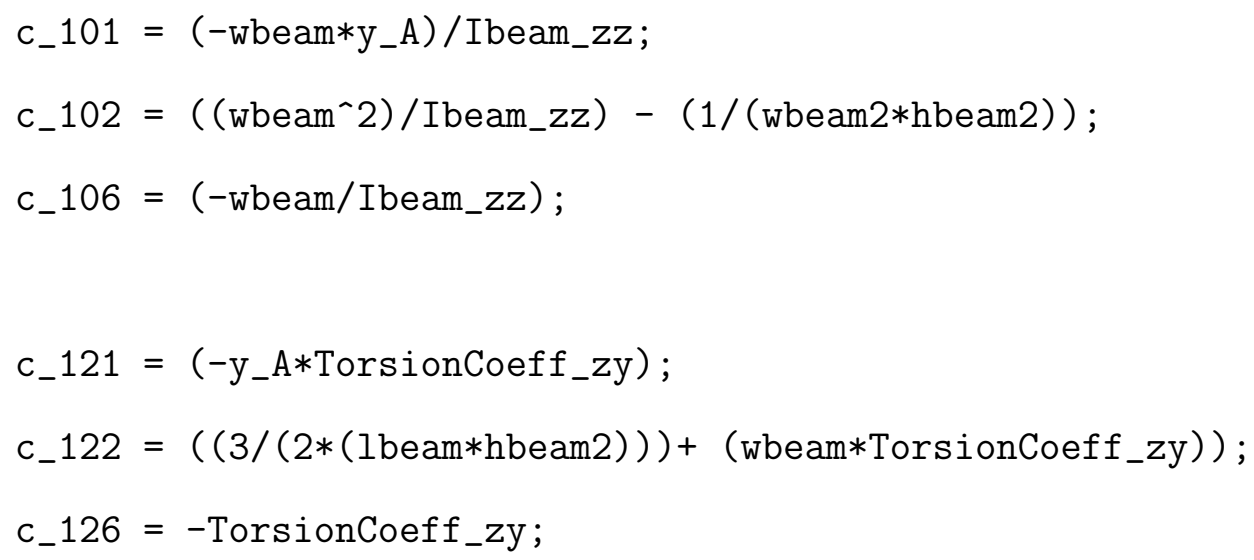


$\%$ the same process can be repeated for y_B $\% \% \mathrm{y} \_\mathrm{B} \% \%$

$\mathrm{d}_{-} 12=\left(\left(-\left(\right.\right.\right.$ hbeam^$\left.\left.^{\wedge} 2\right)\right) /$ Ibeam_xx $)-(1 /($ wbeam $2 *$ hbeam 2$)) ;$

$\mathrm{d}_{-} 13=($ hbeam*y_B $) /$ Ibeam_xx;

$\mathrm{d}_{-} 14=(-$ hbeam $) /$ Ibeam_xx;

d_31 $=((3 / 2) *(1 /$ wbeam $2 *$ lbeam $))+($ hbeam $*$ TorsionCoeff_xy $) ;$

$\mathrm{d}_{-} 35=$-TorsionCoeff_xy;

$\mathrm{d}_{-} 42=\left(\left(-\left(\right.\right.\right.$ hbeam² $\left.\left.^{\wedge}\right)\right) /$ Ibeam_xx $)-(1 /($ wbeam2*hbeam2 $)) ;$

d_43 $=($-hbeam*y_B $) /$ Ibeam_xx;

$\mathrm{d}_{-} 44=($ hbeam $) /$ Ibeam_xx;

d_61 $=((3 / 2) *(1 /$ wbeam $2 *$ lbeam $))+(-$ hbeam $*$ TorsionCoeff_xy $)$;

$d_{-} 65=-$ TorsionCoeff_xy;

$\mathrm{d}_{-} 71=($ wbeam*y_B $) /$ Ibeam_zz;

$\mathrm{d}_{-} 72=(($ wbeam² $) /$ Ibeam_zz $)-(1 /($ wbeam $2 *$ hbeam 2$)) ;$

$\mathrm{d}_{-} 76=($ wbeam $/$ Ibeam_zz $)$;

d_91 = (-y_B*TorsionCoeff_zy) ;

$\mathrm{d}_{-} 92=((3 /(2 *(1$ beam*wbeam 2$)))-($ wbeam*TorsionCoeff_zy $)) ;$

d_96 = -TorsionCoeff_zy; 


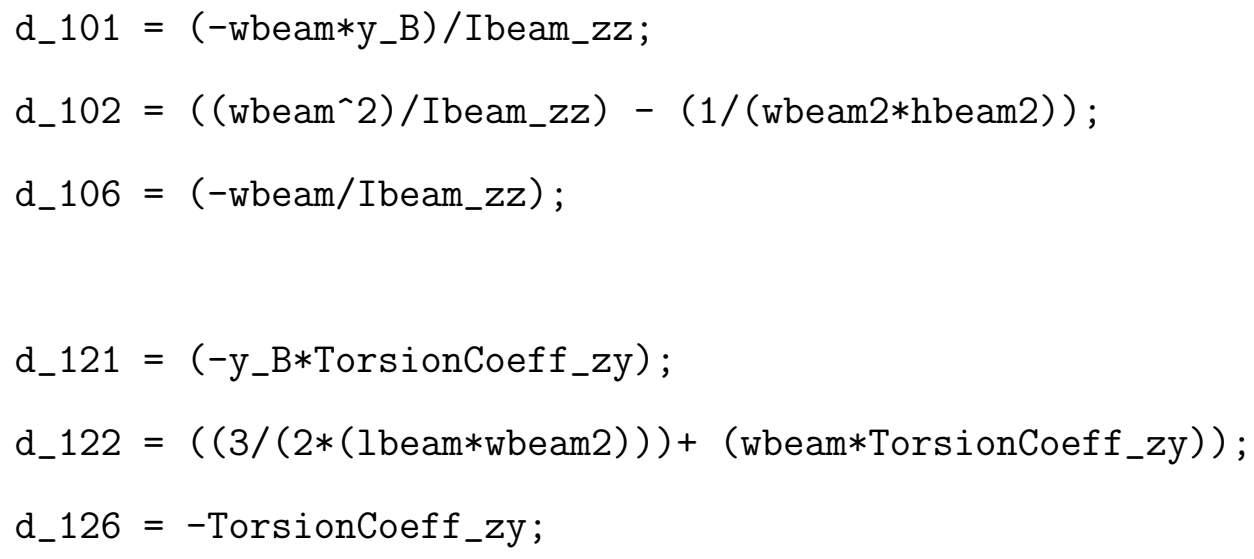




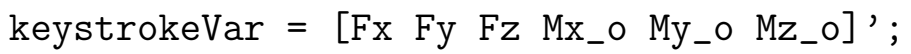

\% \% \% \% \% \% \% \% \% \% \% \% \% \% \% \% \% \% \% \% \% \% \% \% \% \% \% \% \% \% \% \% \% \% \% \% \% \% \% \% \% \% \% \% \% \% \% \% \% \% \% \% \% \% \% \% \% \% \% \% \% \% \% \% \% \% \% \% \% \% \% \% \% \% \% \% \% \% \% \% \%

$\% \% \% \%$ Matrix Assessment $\% \% \% \%$

$\%$ development of stress matrix of directional stresses [12]

stress_yA $=$ coeff_A $*$ keystrokeVar;

stress_yB $=$ coeff_B $*$ keystrokeVar;

$\%$ convert the directional stresses to principal stresses [8]

$\% \%$ Reference [Eq'n 4.16, pg. 156, Fundamentals of Machine Component Design,

5th ed., Juvinall and Marshek]

$\% \%$ note: sigma_1 > sigma_2

sigma_1_yA $=\operatorname{zeros}(4,1)$;

sigma_2_yA $=$ zeros $(4,1)$;

sigma_1_yB $=\operatorname{zeros}(4,1)$;

sigma_2_yB $=\operatorname{zeros}(4,1)$;

theta2_yA $=\operatorname{zeros}(4,1)$;

theta2_yB $=\operatorname{zeros}(4,1)$;

$i=1$;

$j=1$;

for $j=1: 4$

sigma_1_yA $(j,:)=\left(\operatorname{stress} \_y A(i,:)+\operatorname{stress}_{-} y A((i+1),:)\right) / 2+$

$\operatorname{sqrt}\left(\left(\left(\operatorname{stress}_{-} y A((i+2),:)\right) \wedge 2\right)+\left(\left(\left(\operatorname{stress}_{-} y A(i,:)-\operatorname{stress} \_y A((i+1),:)\right) / 2\right) \wedge 2\right)\right)$;

sigma_2_yA $(j,:)=\left(\operatorname{stress} \_y A(i,:)+\operatorname{stress}_{-} y A((i+1),:)\right) / 2-$

$\operatorname{sqrt}\left(\left(\left(\operatorname{stress}_{-} \mathrm{yA}((i+2),:)\right) \wedge 2\right)+\left(\left(\left(\operatorname{stress}_{-} y A(i,:)-\operatorname{stress_{-}yA}((i+1),:)\right) / 2\right) \wedge 2\right)\right)$;

sigma_1_yB $(j,:)=\left(\operatorname{stress}_{-} y B(i,:)+\operatorname{stress}_{-} y B((i+1),:)\right) / 2+$ 


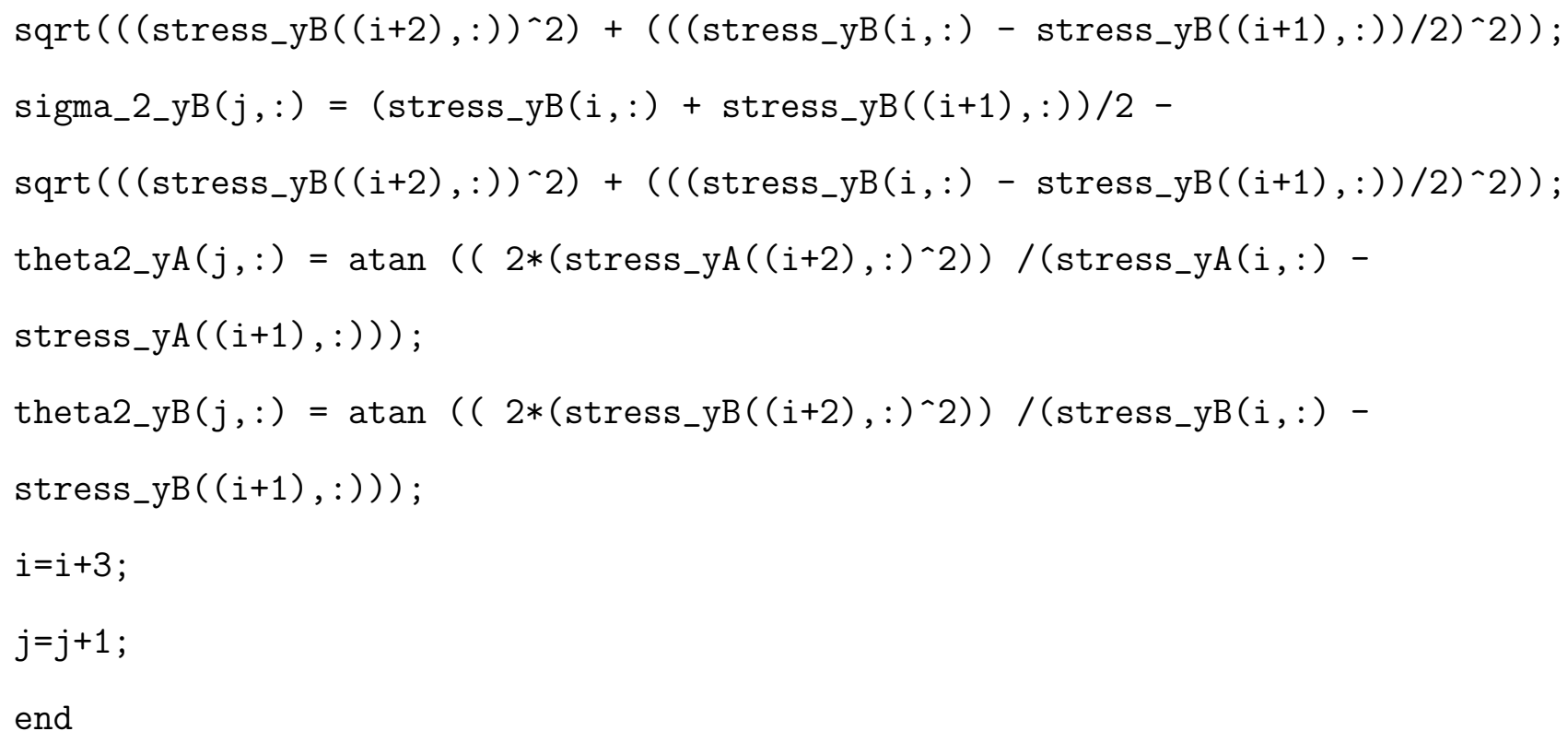


\%convert principal strains into directional strains [12]

$\%$ Derived from Reference [Eq'n 5.3 and 5.4, pg. 200,

Fundamentals of Machine Component Design, 5th ed., Juvinall and Marshek]

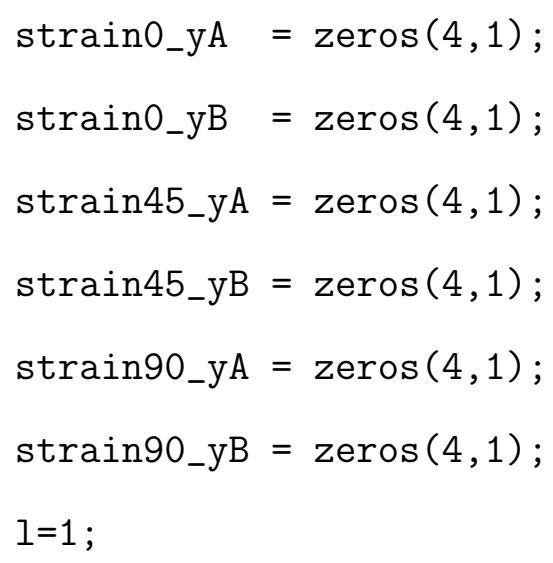

for $1=1: 4$

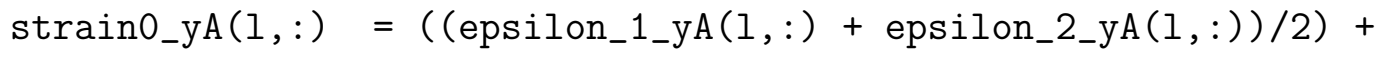

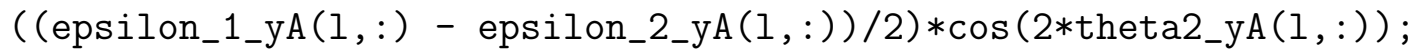

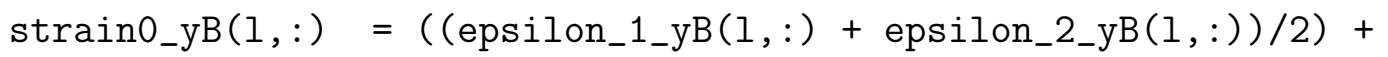
((epsilon_1_yB( $(1,:)$ - epsilon_2_yB( $1,:)) / 2) * \cos (2 * \operatorname{theta2}$ yB $(1,:))$; strain45_yA(1,:) = ((epsilon_1_yA(1,:) + epsilon_2_yA(1,:))/2) + $\left(\left(\operatorname{epsilon} \_1 \_y A(1,:)\right.\right.$ - epsilon_2_yA $\left.\left.(1,:)\right) / 2\right) * \cos \left(2 *\left(\operatorname{theta} 2 \_y A(1,:)+45\right)\right)$;

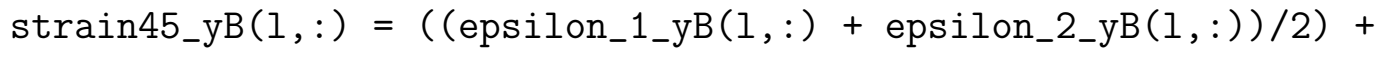
$\left(\left(\operatorname{epsilon} \_1 \_y B(1,:)\right.\right.$ - epsilon_2_yB $\left.\left.(1,:)\right) / 2\right) * \cos \left(2 *\left(\operatorname{theta} 2 \_y B(1,:)+45\right)\right)$;

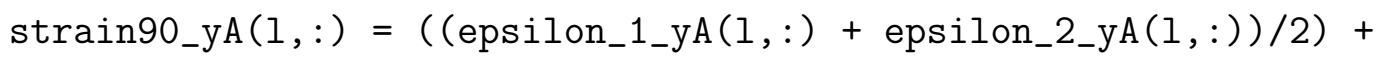
$(($ epsilon_1_yA(1,: ) - epsilon_2_yA $(1,:)) / 2) * \cos \left(2 *\left(\operatorname{theta} 2 \_y A(1,:)+90\right)\right)$; strain90_yB(1,:) = ((epsilon_1_yB(l,: ) + epsilon_2_yB(l,:))/2) + $(($ epsilon_1_yB( $(1,:)$ - epsilon_2_yB $(1,:)) / 2) * \cos (2 *($ theta2_yB $(1,:)+90))$; $1=1+1$ end 
$\% \% \% \%$ Rosette Assessment $\% \% \% \%$

$\%$ direct readings from single rosettes

microstrain0_yA = strain0_yA*(10^6);

microstrain0_yB = strain0_yB*(10^6);

microstrain45_yA = strain45_yA*(10^6);

microstrain45_yB = strain45_yB*(10^6);

microstrain90_yA = strain90_yA*(10^6);

microstrain90_yB = strain90_yB*(10^6);

$\%$ what gauge change would we see?

$\%$ percentchange0_yA $=$ GF* strain0_yA $* 100 ;$

$\%$ percentchange0_yB = GF* strain0_yB* 100;

$\% \quad$ percentchange45_yA $=$ GF* strain45_yA* 100;

$\% \quad$ percentchange45_yB $=$ GF* strain45_yB* 100;

$\%$ percentchange90_yA $=$ GF* strain90_yA $* 100 ;$

$\% \quad$ percentchange90_yB $=$ GF* strain90_yB* 100;

$\%$

$\% \quad$ Rchange0_yA = Rnom* percentchange0_yA / 100;

$\% \quad$ Rchange0_yB = Rnom* percentchange0_yB /100;

$\% \quad$ Rchange45_yA $=$ Rnom* percentchange45_yA /100;

$\% \quad$ Rchange45_yB = Rnom* percentchange45_yB /100;

$\% \quad$ Rchange90_yA $=$ Rnom* percentchange90_yA /100;

$\% \quad$ Rchange90_yB = Rnom* percentchange90_yB /100; 
$\%$ to amplify the readings and reduce the number of inputs, bridges can be applied

$\%$ half-bridge pairs can be created with pairs from both Plane A and Plane B strain_bridgepair_r12_0_yA = [strain0_yA(1); strain0_yA(2)];

strain_bridgepair_r12_0_yB = [strain0_yB(1); strain0_yB(2)];

strain_bridgepair_r12_45_yA = [strain45_yA(1); strain45_yA(2)];

strain_bridgepair_r12_45_yB = [strain45_yB(1); strain45_yB(2)];

strain_bridgepair_r12_90_yA = [strain90_yA(1); strain90_yA(2)];

strain_bridgepair_r12_90_yB = [strain90_yB(1); strain90_yB(2)];

strain_bridgepair_r34_0_yA = [strain0_yA(3); strain0_yA(4)];

strain_bridgepair_r34_0_yB = [strain0_yB(3); strain0_yB(4)];

strain_bridgepair_r34_45_yA = [strain45_yA(3); strain45_yA(4)];

strain_bridgepair_r34_45_yB = [strain45_yB(3); strain45_yB (4)];

strain_bridgepair_r34_90_yA = [strain90_yA(3); strain90_yA(4)];

strain_bridgepair_r34_90_yB = [strain90_yB(3); strain90_yB(4)];

$\%$ now that the pairs are developed, a bridge can be created with the pairs

$\%$ and two reference resistors

$\%$ the voltage output of the bridge can be calculated

Vout_yA = zeros $(6,1)$;

Vout_yB = zeros $(6,1)$;

$\%$ the new resistance can be defined as

$\% \%$ Derived from Reference [Eq'n 9.21, pg. 397,

Introduction to Mechantronics and Measuring Systems, 4th Ed., 


$$
\begin{aligned}
& \text { Alciatore and Histand] } \\
& \text { R_p_r12_0_yA = (GF* strain_bridgepair_r12_0_yA(1) *Rnom) }+ \text { Rnom; } \\
& \text { R_q_r12_0_yA = (GF* strain_bridgepair_r12_0_yA(2) *Rnom) }+ \text { Rnom; } \\
& \text { R_p_r12_45_yA = (GF* strain_bridgepair_r12_45_yA (1)*Rnom) }+ \text { Rnom; } \\
& \text { R_q_r12_45_yA = (GF* strain_bridgepair_r12_45_yA (2)*Rnom) }+ \text { Rnom; } \\
& \text { R_p_r12_90_yA = (GF* strain_bridgepair_r12_90_yA (1) *Rnom) }+ \text { Rnom; } \\
& \text { R_q_r12_90_yA = (GF* strain_bridgepair_r12_90_yA (2) *Rnom) }+ \text { Rnom; } \\
& \text { R_p_r34_0_yA = (GF* strain_bridgepair_r34_0_yA (1) } * \text { Rnom })+ \text { Rnom; } \\
& \text { R_q_r34_0_yA = (GF* strain_bridgepair_r34_0_yA (2) *Rnom) }+ \text { Rnom; } \\
& \text { R_p_r34_45_yA = (GF* strain_bridgepair_r34_45_yA (1)*Rnom) }+ \text { Rnom; } \\
& \text { R_q_r34_45_yA = (GF* strain_bridgepair_r34_45_yA (2)*Rnom) + Rnom; } \\
& \text { R_p_r34_90_yA = (GF* strain_bridgepair_r34_90_yA (1)*Rnom) + Rnom; } \\
& \text { R_q_r34_90_yA = (GF* strain_bridgepair_r34_90_yA (2)*Rnom) }+ \text { Rnom; }
\end{aligned}
$$$$
\text { R_p_r12_0_yB = (GF* strain_bridgepair_r12_0_yB(1) *Rnom) }+ \text { Rnom; }
$$$$
\text { R_q_r12_0_yB = (GF* strain_bridgepair_r12_0_yB (2) *Rnom) + Rnom; }
$$$$
\text { R_p_r12_45_yB = (GF* strain_bridgepair_r12_45_yB (1)*Rnom) + Rnom; }
$$$$
\text { R_q_r12_45_yB = (GF* strain_bridgepair_r12_45_yB (2)*Rnom) + Rnom; }
$$$$
\text { R_p_r12_90_yB = (GF* strain_bridgepair_r12_90_yB (1)*Rnom) + Rnom; }
$$$$
\text { R_q_r12_90_yB = (GF* strain_bridgepair_r12_90_yB (2)*Rnom) + Rnom; }
$$$$
\text { R_p_r34_0_yB = (GF* strain_bridgepair_r34_0_yB(1) *Rnom) }+ \text { Rnom; }
$$$$
\text { R_q_r34_0_yB = (GF* strain_bridgepair_r34_0_yB(2) *Rnom) }+ \text { Rnom; }
$$$$
\text { R_p_r34_45_yB = (GF* strain_bridgepair_r34_45_yB (1)*Rnom) + Rnom; }
$$$$
\text { R_q_r34_45_yB = (GF* strain_bridgepair_r34_45_yB (2)*Rnom) + Rnom; }
$$$$
\text { R_p_r34_90_yB = (GF* strain_bridgepair_r34_90_yB (1)*Rnom) + Rnom; }
$$$$
\text { R_q_r34_90_yB = (GF* strain_bridgepair_r34_90_yB (2)*Rnom) + Rnom; }
$$ 
$\%$ in $[\mathrm{mV}]$

Vout_r12_0_yA $=\operatorname{Vex} *\left(\left(\left(R_{-}\right.\right.\right.$p_r12_0_yA $*$ Rnom $)-($ Rnom*R_q_r12_0_yA $\left.)\right) /$

$(($ R_p_r12_0_yA+R_q_r12_0_yA $) *($ Rnom+Rnom $))$ );

Vout_r12_45_yA $=\operatorname{Vex} *\left(\left(\left(R_{-}\right.\right.\right.$P_r12_45_yA $*$ Rnom $)-($ Rnom*R_q_r12_45_yA $\left.)\right) /$

$(($ R_p_r12_45_yA+R_q_r12_45_yA $) *($ Rnom+Rnom $)))$;

Vout_r12_90_yA $=\operatorname{Vex} *\left(\left(\left(R_{-}\right.\right.\right.$p_r12_90_yA*Rnom $)-($Rnom*R_q_r12_90_yA $\left.)\right) /$

$(($ R_p_r12_90_yA+R_q_r12_90_yA $) *($ Rnom+Rnom $))$ ) ;

Vout_r34_0_yA $=\operatorname{Vex} *\left(\left(\left(R_{-}\right.\right.\right.$p_r34_0_yA $*$ Rnom $)-($ Rnom*R_q_r34_0_yA $\left.)\right) /$

$\left(\left(R_{-}\right.\right.$p_r34_0_yA+R_q_r34_0_yA $) *($ Rnom+Rnom $\left.)\right)$ );

Vout_r34_45_yA $=\operatorname{Vex} *\left(\left(\left(R_{-}\right.\right.\right.$P_r34_45_yA $*$ Rnom $)-($ Rnom*R_q_r34_45_yA $\left.)\right) /$

$\left(\left(R_{-}\right.\right.$p_r34_45_yA+R_q_r34_45_yA $) *($ Rnom+Rnom $\left.)\right)$ );

Vout_r34_90_yA $=\operatorname{Vex} *\left(\left(\left(R_{-}\right.\right.\right.$p_r34_90_yA $*$ Rnom $)-($ Rnom*R_q_r34_90_yA $\left.)\right) /$

((R_p_r34_90_yA+R_q_r34_90_yA)*(Rnom+Rnom) ) );

Vout_r12_0_yB $=\operatorname{Vex} *\left(\left(\left(R_{-}\right.\right.\right.$p_r12_0_yB*Rnom $)-($Rnom*R_q_r12_0_yB $\left.)\right) /$

$\left(\left(R_{-}\right.\right.$p_r12_0_yB+R_q_r12_0_yB $) *($ Rnom+Rnom $\left.)\right)$ );

Vout_r12_45_yB = Vex $*\left(\left(\left(R_{-}\right.\right.\right.$p_r12_45_yB*Rnom $)-($Rnom*R_q_r12_45_yB $\left.)\right) /$

$\left(\left(R_{-}\right.\right.$p_r12_45_yB+R_q_r12_45_yB $) *($ Rnom+Rnom $\left.)\right)$ );

Vout_r12_90_yB $=\operatorname{Vex} *\left(\left(\left(R_{-}\right.\right.\right.$p_r12_90_yB*Rnom $)-($Rnom*R_q_r12_90_yB $\left.)\right) /$

((R_p_r12_90_yB+R_q_r12_90_yB)*(Rnom+Rnom $))$ ) ;

Vout_r34_0_yB $=\operatorname{Vex} *\left(\left(\left(R_{-}\right.\right.\right.$p_r34_0_yB*Rnom $)-($Rnom*R_q_r34_0_yB $\left.)\right) /$

$\left(\left(R_{-}\right.\right.$p_r34_0_yB+R_q_r34_0_yB $) *($ Rnom+Rnom $\left.)\right)$ );

Vout_r34_45_yB $=\operatorname{Vex} *\left(\left(\left(R_{-}\right.\right.\right.$p_r34_45_yB*Rnom $)-($Rnom*R_q_r34_45_yB $\left.)\right) /$

$\left(\left(R_{-}\right.\right.$p_r34_45_yB+R_q_r34_45_yB $) *($ Rnom+Rnom $\left.)\right)$ );

Vout_r34_90_yB = Vex $*\left(\left(\left(R \_p \_r 34 \_90 \_y B * R n o m\right)-\left(R n o m * R \_q \_r 34 \_90 \_y B\right)\right) /\right.$

((R_p_r34_90_yB+R_q_r34_90_yB $) *($ Rnom+Rnom $))$ );

end 
Appendix E

Prototype Key Components 

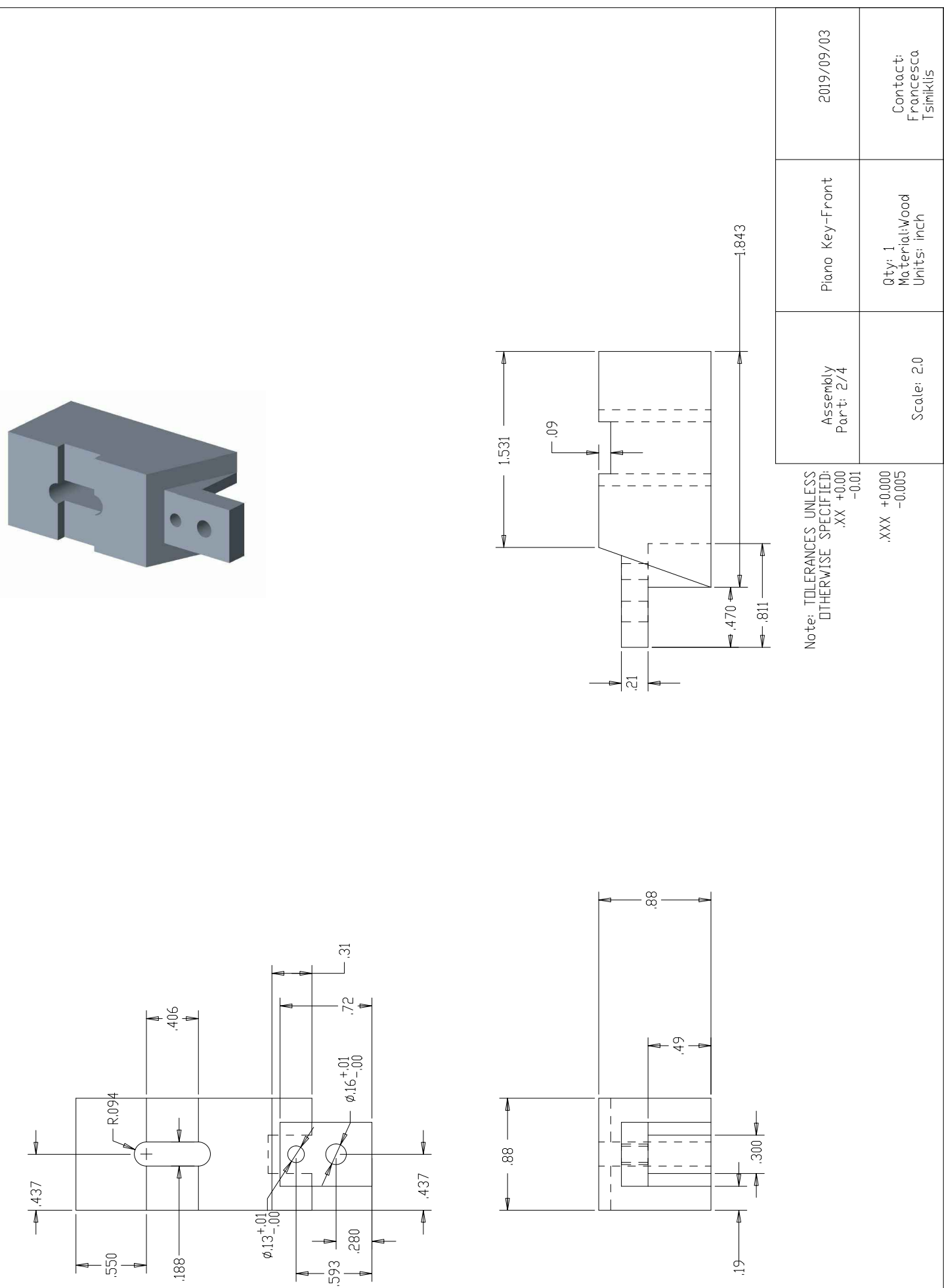

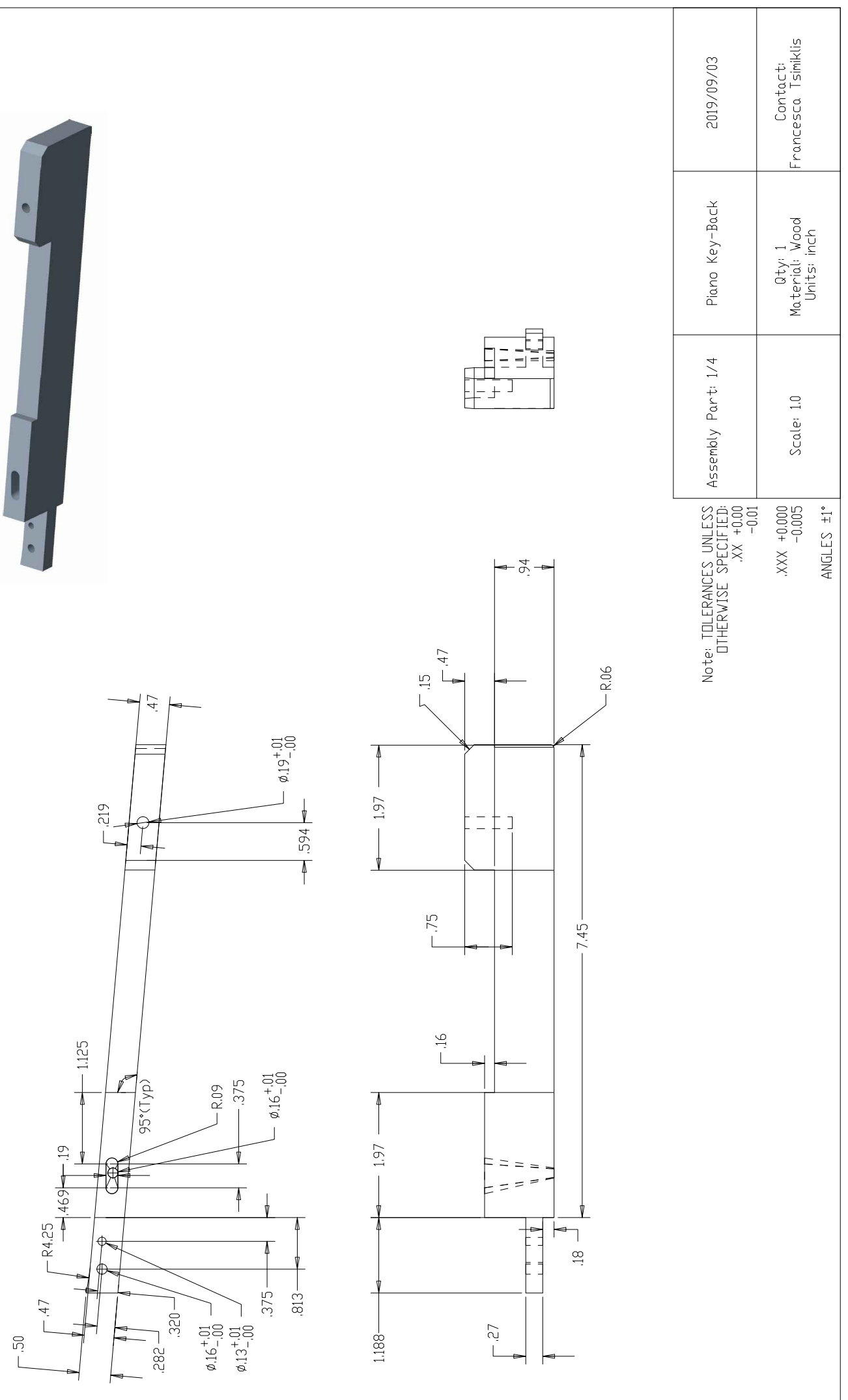


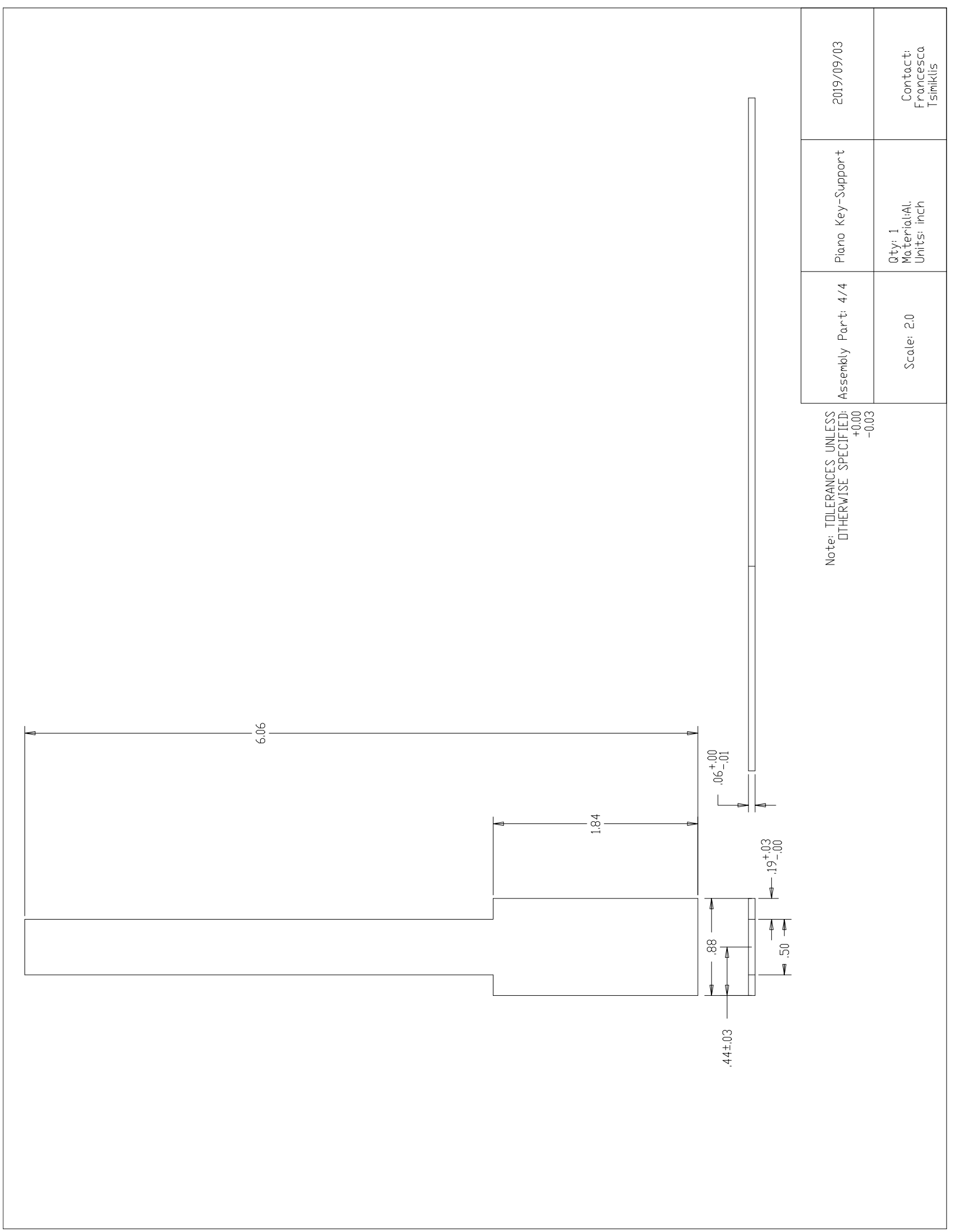




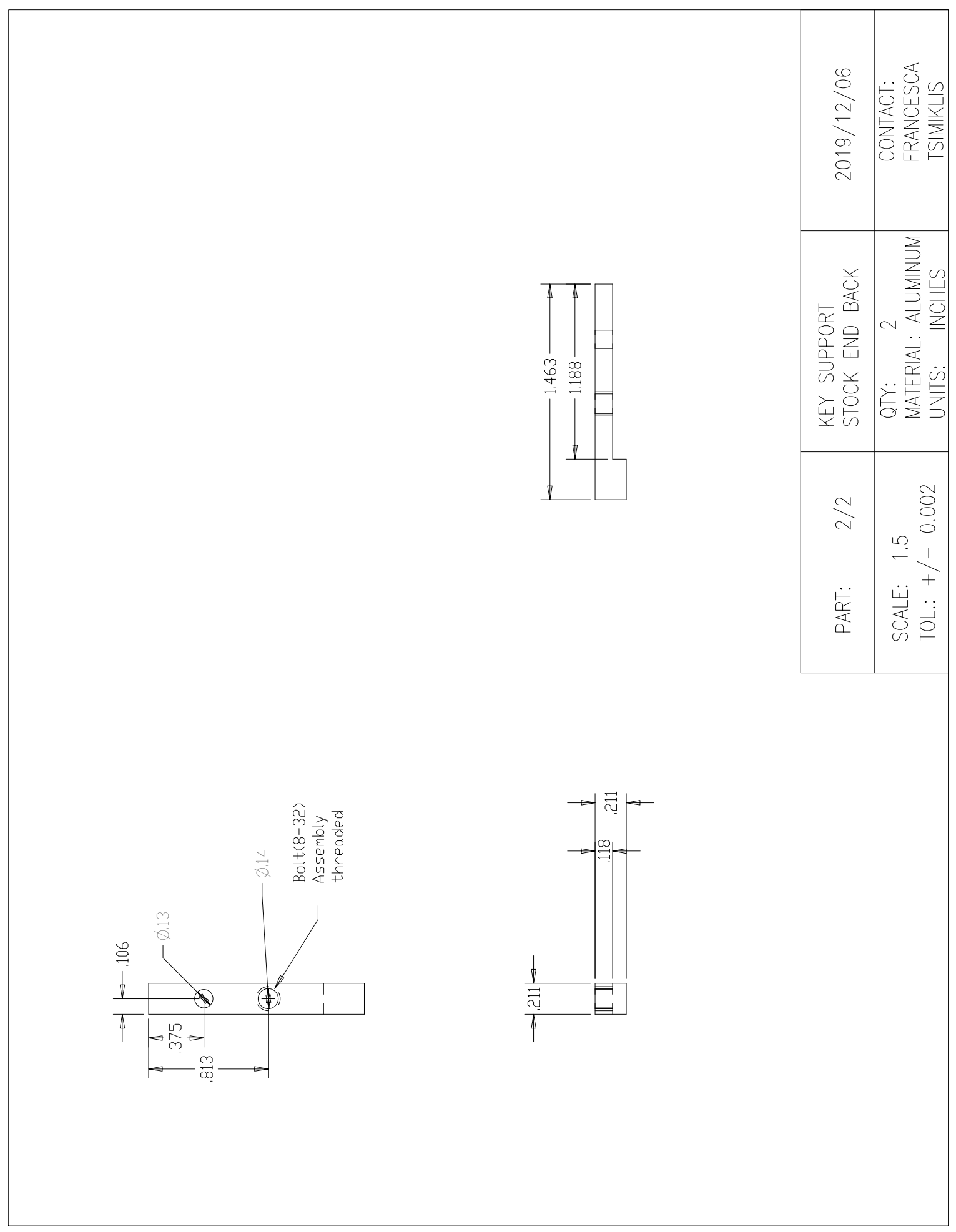




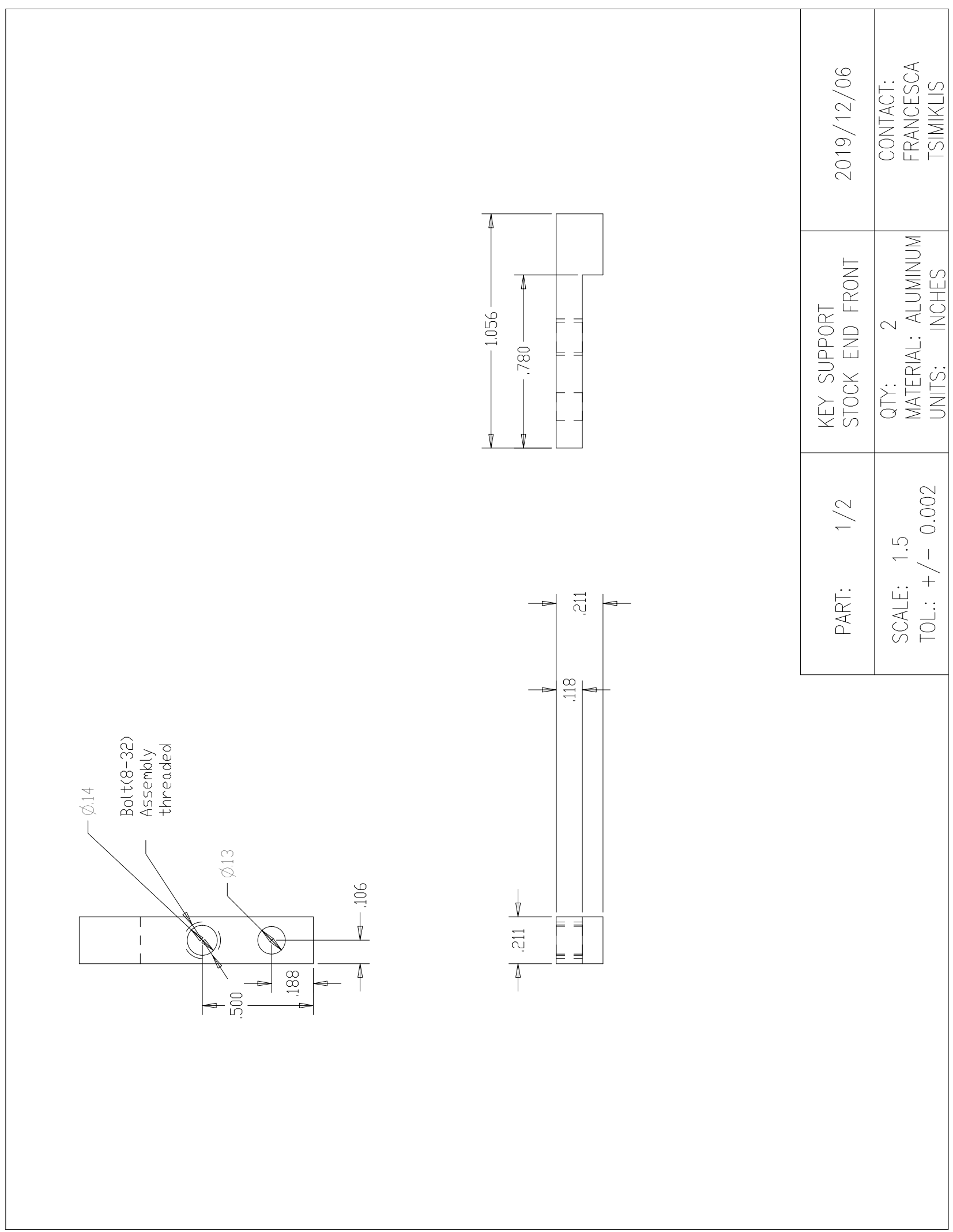




\section{Appendix F}

\section{Code Listing: LabJack Data Collection Script}

This appendix contains the code used to communicate with the LabJack to complete data collection. This code was developed in the LJM Matlab Library from LabJack, and was edited for this application.

\%script edited from LJM Matlab library from LabJack

\%ensure main folder and subfolders are added to the path, ...

\%allowing MATLAB library to be accessible

close all

clear all

\%Make connected LabJack visible

ljmAsm = NET.addAssembly ('LabJack.LJM');

\%Creating an object to nested class LabJack. LJM.CONSTANTS

$t=1 \mathrm{jmAsm}$. AssemblyHandle.GetType ('LabJack.LJM+CONSTANTS');

LJM_CONSTANTS $=$ System.Activator.CreateInstance $(t)$;

handle $=0$; 
try

\%Open T7 device, Any connection, Any identifier

[ljmError, handle] = LabJack.LJM.OpenS('T7', 'ANY', 'ANY', handle); showDeviceInfo(handle)

$\%$ define the number of scans to run and number of input channels to read from maxRequests $=10000 ; \%$ the number of stream loops that will run scanRate $=$ double $(2000) ; \%$ Scans per second scansPerRead $=\operatorname{int32}($ scanRate $)$; numAINs $=12 ; \%$ number of input channels to read firstAINChan $=0 ; \%$ Starting AIN channel. $0=$ AINO. numAddresses = numAINs;

$\%$ Scan list names to stream aScanListNames = NET. createArray ('System.String', numAddresses); for $i=1:$ numAddresses

chan $=$ firstAINChan $+i-1$ $\operatorname{aScanListNames}(i)=[$ 'AIN' num2str $($ chan $)]$;

end

$\%$ Scan list addresses to stream aScanList $=$ NET.createArray ('System.Int32', numAddresses); \% Dummy array for aTypes parameter aTypes = NET.createArray ('System.Int32', numAddresses); LabJack.LJM.NamesToAddresses (numAddresses, aScanListNames, aScanList, aTypes);

$\%$ Stream reads will be stored in aData. Needs to be at least $\%$ numAddresses*scansPerRead in size. 
aData $=$ NET. createArray ('System. Double', numAddresses*scansPerRead);

$\operatorname{try}$

$\%$ When streaming, negative channels and ranges can be configured for $\%$ individual analog inputs, but the stream has only one settling time $\%$ and resolution.

\% Ensure triggered stream is disabled.

LabJack.LJM.eWriteName (handle, 'STREAM_TRIGGER_INDEX', 0); \% Enabling internally-clocked stream.

LabJack.LJM.eWriteName (handle, 'STREAM_CLOCK_SOURCE' , 0) ;

\% All negative channels are single-ended, ranges are

$\%+/-10 \mathrm{~V}$, stream settling is 0 (default) and stream resolution index $\%$ is 0 (default).

numFrames $=5$;

aNames = NET.createArray('System.String', numFrames);

$\operatorname{aNames}(1)=$ 'AIN_ALL_NEGATIVE_CH' ;

$\operatorname{aNames}(2)=$ 'AINO_RANGE' ;

$\operatorname{aNames}(3)=$ 'AIN1_RANGE';

$\operatorname{aNames}(4)=$ 'STREAM_SETTLING_US' ;

$\operatorname{aNames}(5)=$ 'STREAM_RESOLUTION_INDEX';

aValues = NET. createArray('System.Double', numFrames);

$\operatorname{aValues}(1)=$ LJM_CONSTANTS.GND;

aValues $(2)=10.0 ; \% \mathrm{x} 1$ internal amplication, voltage input range of $+/-10 \mathrm{~V}$

aValues $(3)=10.0 ; \% x 1$ internal amplication, voltage input range of $+/-10 \mathrm{~V}$

$\operatorname{aValues}(4)=0$;

$\operatorname{aValues}(5)=0$;

$\%$ Write the analog inputs' negative channels (when applicable), ranges 
$\%$ stream settling time and stream resolution configuration.

LabJack.LJM.eWriteNames (handle, numFrames, aNames, aValues, -1);

$\%$ Configure and start stream

numAddresses = aScanList. Length;

$[\sim$, scanRate $]=$ LabJack.LJM.eStreamStart (handle, scansPerRead, . .

numAddresses, aScanList, scanRate);

disp(['Stream started with a scan rate of ' num2str(scanRate) 'Hz.'])

tic

disp(['Performing ' num2str(maxRequests)', stream reads.'])

totalScans $=0$;

curSkippedSamples $=0$;

totalSkippedSamples $=0$;

$k=1$;

for $i=1:$ maxRequests

[ , devScanBL, ljmScanBL] = LabJack.LJM.eStreamRead(handle, aData, 0, 0);

totalScans = totalScans + scansPerRead;

\% Count the skipped samples which are indicated by -9999

$\%$ values. Skipped samples occur after a device's stream buffer

$\%$ overflows and are reported after auto-recover mode ends.

$\%$ When streaming at faster scan rates in MATLAB, try counting 
$\%$ the skipped packets outside your eStreamRead loop if you are $\%$ getting skipped samples/scan.

curSkippedSamples $=\operatorname{sum}(\operatorname{double}($ aData $)==-9999.0)$;

totalSkippedSamples = totalSkippedSamples + curSkippedSamples;

mydata $(:, i)=\left(\operatorname{double}(\text { aData })^{\prime}\right)$;

$\%$ reshape data to arrange the voltage readings to have each

$\%$ voltage channel as a separate column

mydatareshapped $=(\operatorname{reshape}(\operatorname{mydata}(:, i),[\text { numAINs }, \text { scansPerRead }]))^{\prime}$;

voltageoutputsraw $(\mathrm{k}: \mathrm{k}+($ scansPerRead -1$), 1:$ numAINs $)=$ mydatareshapped;

$\mathrm{k}=\mathrm{k}+\mathrm{scansPerRead}$;

end

timeElapsed = toc;

$\operatorname{disp}([$ 'Total scans $=$ 'num2str(totalScans) $])$

$\operatorname{disp}([$ 'Skipped Scans $=$ ' num2str(totalSkippedSamples/numAddresses)])

$\operatorname{disp}([$ 'Time Taken $=$ ' num2str(timeElapsed)', seconds'])

$\operatorname{disp}(['$ LJM Scan Rate =' num2str(scanRate)', scans/second'])

$\operatorname{disp}([$ 'Timed Scan Rate =' num2str(totalScans/timeElapsed) ' scans/second'])

$\operatorname{disp}([$ 'Sample Rate $=$ ' num2str(numAddresses*totalScans/timeElapsed) , ...

samples/second'] )

catch e

showErrorMessage (e)

end

disp('Stop Stream')

LabJack. LJM. eStreamStop (handle); 
\% Close handle

LabJack. LJM. Close (handle);

catch e

showErrorMessage (e)

LabJack. LJM.CloseAll();

return

end

\%now use voltageoutputsraw with the previously determined calibration process \%for example a pre-determined q matrix for a pseudo-inverse matrix, $\%$ could be applied to estimate the user inputs

$\mathrm{v}=$ voltageoutputsraw;

$\%$ ie. $q=$ [q_pinv_Fx, q_pinv_Fy, q_pinv_Fz, q_pinv_Mx, q_pinv_My, q_pinv_Mz] $\mathrm{F}=\mathrm{v}^{*} \mathrm{q}^{\prime}$

$\%$ save the values of this test in a c.csv

outputs $=\mathrm{F}$;

filename = 'DataCollection_ForceReadings.csv';

dlmwrite(filename, outputs, 'delimiter' , ', ' , '-append') 


\section{Appendix G}

\section{Data Analysis: Experimental Data \\ Collection}

\section{G.1 Measurement Positions}

The exact position and angle input specifications for each loading case were recorded. These positions were defined with respect ot the keytop surface, shown again in Figure G.1.

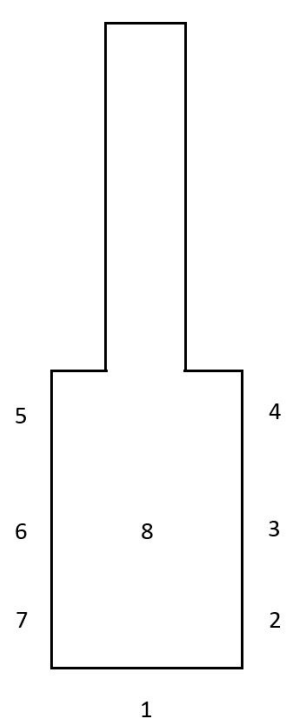

Figure G.1: Defined Positions of Measurement on the Experimental Keytop 
Table G.1: Position of $F_{z}$ Force Tests

\begin{tabular}{|l|l|}
\hline Position Number & Position Coordinate (mm) \\
\hline 1 & $(0,0,16.175)$ \\
\hline 2 & $(11,8,16.175)$ \\
\hline 3 & $(11,23,16.175)$ \\
\hline 4 & $(11,44,16.175)$ \\
\hline 5 & $(-11,44,16.175)$ \\
\hline 6 & $(-11,23,16.175)$ \\
\hline 7 & $(-11,8,16.175)$ \\
\hline 8 & $(0,23,16.175)$ \\
\hline
\end{tabular}

Table G.2: Position of $F_{x}$ Force Tests

\begin{tabular}{|c|l|c|}
\hline Loading Case Number & $\begin{array}{l}\text { Position of } \\
\text { Load Applica- } \\
\text { tion }\end{array}$ & Position Coordinate (mm) \\
\hline 1 & 8 & $(0,23,16.175)$ \\
\hline 2 & 8 & $(0,23,16.175)$ \\
\hline
\end{tabular}

Table G.3: Position of Combined $F_{z}$ and $F_{y}$ Force Tests

\begin{tabular}{|l|l|l|l|}
\hline $\begin{array}{l}\text { Loading Case } \\
\text { Number }\end{array}$ & $\begin{array}{l}\text { Angle of Load } \\
\text { Application }\left(^{\circ}\right)\end{array}$ & $\begin{array}{l}\text { Position of Load } \\
\text { Application }\end{array}$ & $\begin{array}{l}\text { Position Coordi- } \\
\text { nate (mm) }\end{array}$ \\
\hline 1 & -90 & 1 & $(0,0,16.175)$ \\
\hline 2 & -57.5 & 1 & $(0,0,16.175)$ \\
\hline 3 & -45 & 1 & $(0,0,16.175)$ \\
\hline 4 & 13 & base of key & $(0,3,-10.175)$ \\
\hline 5 & 45 & base of key & $(0,3,-10.175)$ \\
\hline 6 & 90 & base of key & $(0,3,-10.175)$ \\
\hline
\end{tabular}


Table G.4: Precise Mass Applied During Each Loading Case

\begin{tabular}{|c|c|c|}
\hline & \multicolumn{2}{|c|}{ Mass Applied (kg) } \\
\hline Case Number & $F_{z}$ Testing & $F_{x}$ and $F_{y}$ Testing \\
\hline 1 & 0 & 0 \\
\hline 2 & 0.03301 & 0.03301 \\
\hline 3 & 0.05013 & 0.05013 \\
\hline 4 & 0.05128 & 0.05128 \\
\hline 5 & 0.05242 & 0.05242 \\
\hline 6 & 0.5296 & 0.5296 \\
\hline 7 & 0.05407 & 0.05407 \\
\hline 8 & 0.05521 & 0.05521 \\
\hline 9 & 0.05635 & 0.05635 \\
\hline 10 & 0.05702 & 0.05702 \\
\hline 11 & 0.05814 & 0.05814 \\
\hline 12 & 0.05927 & 0.05927 \\
\hline 13 & 0.0604 & 0.0604 \\
\hline 14 & 0.10000 & 0.10000 \\
\hline 15 & 0.20002 & 0.15000 \\
\hline 16 & 0.30003 & 0.20000 \\
\hline 17 & 0.40002 & 0.25000 \\
\hline 18 & 0.50001 & - \\
\hline 19 & 0.60001 & - \\
\hline 20 & 0.70002 & - \\
\hline 21 & 0.80002 & - \\
\hline 22 & 0.90004 & - \\
\hline 23 & 1.00004 & - \\
\hline
\end{tabular}




\section{G.2 Plotted Voltage Outputs}

G.2.1 Voltage Outputs from Fz and Fy Loading Cases 


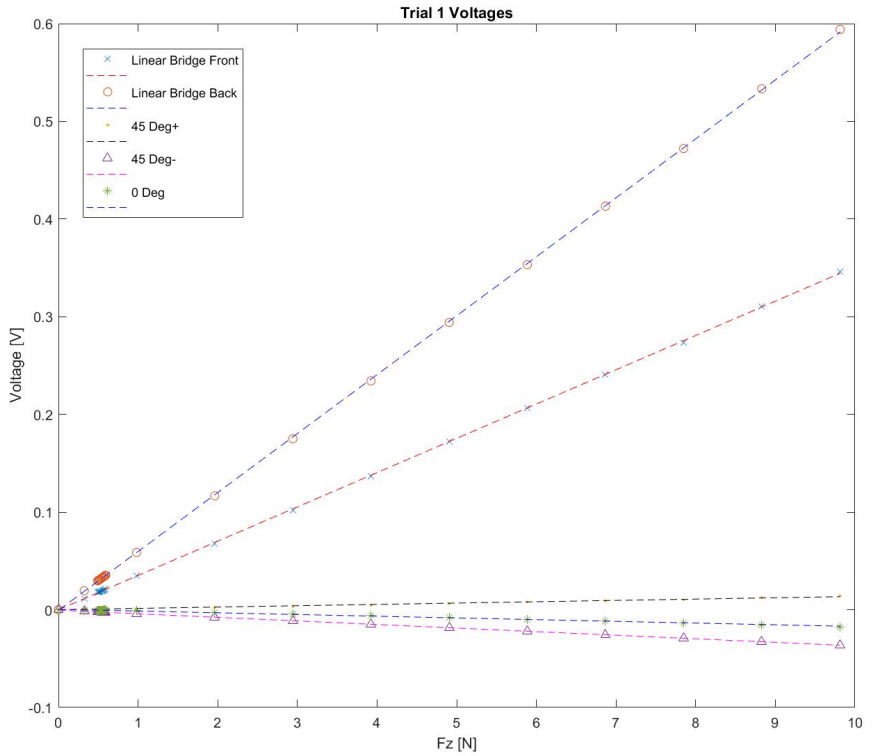

(a) Trial 1

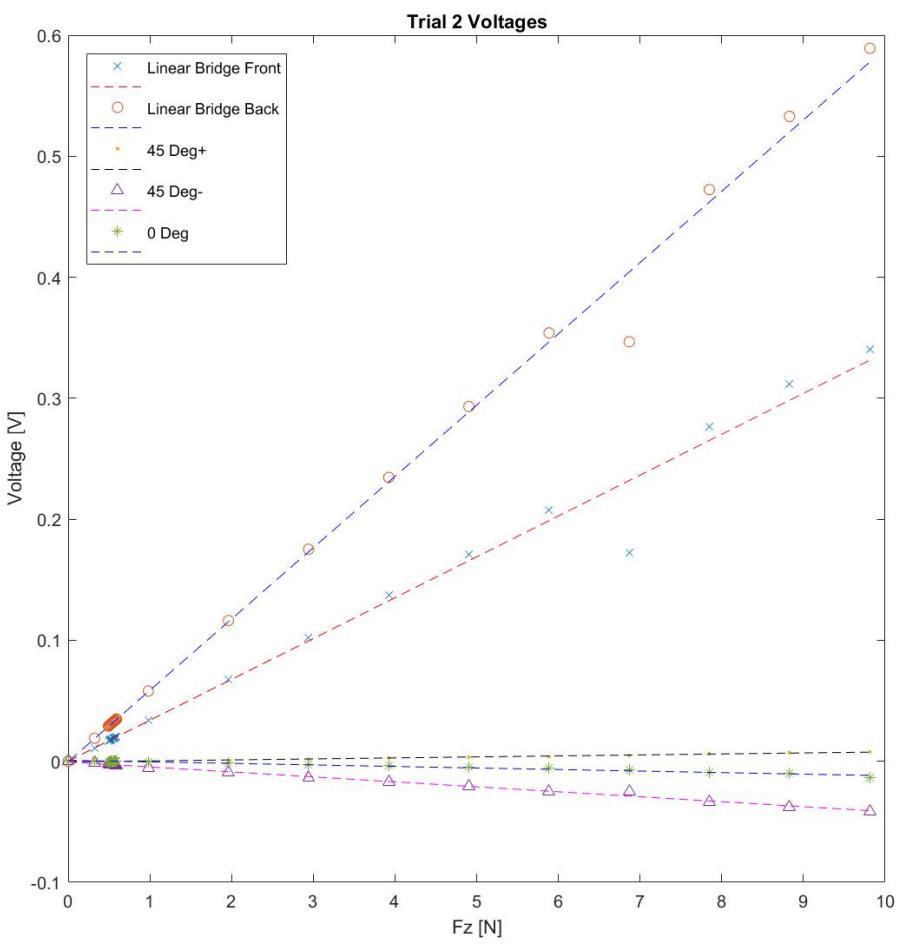

(b) Trial 2 


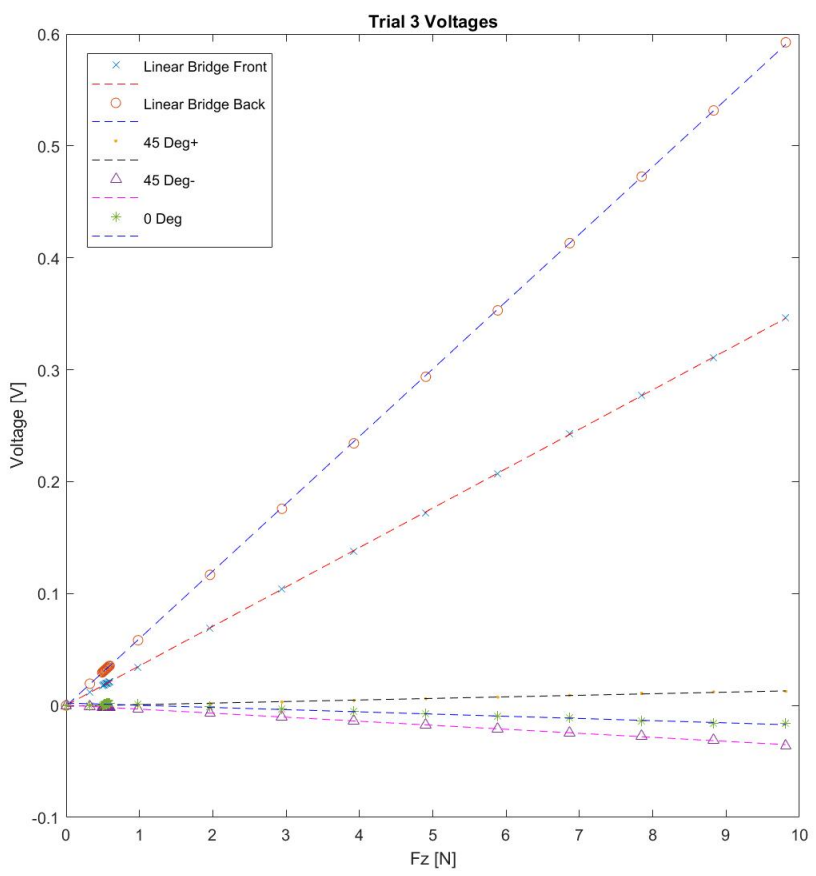

(c) Trial 3

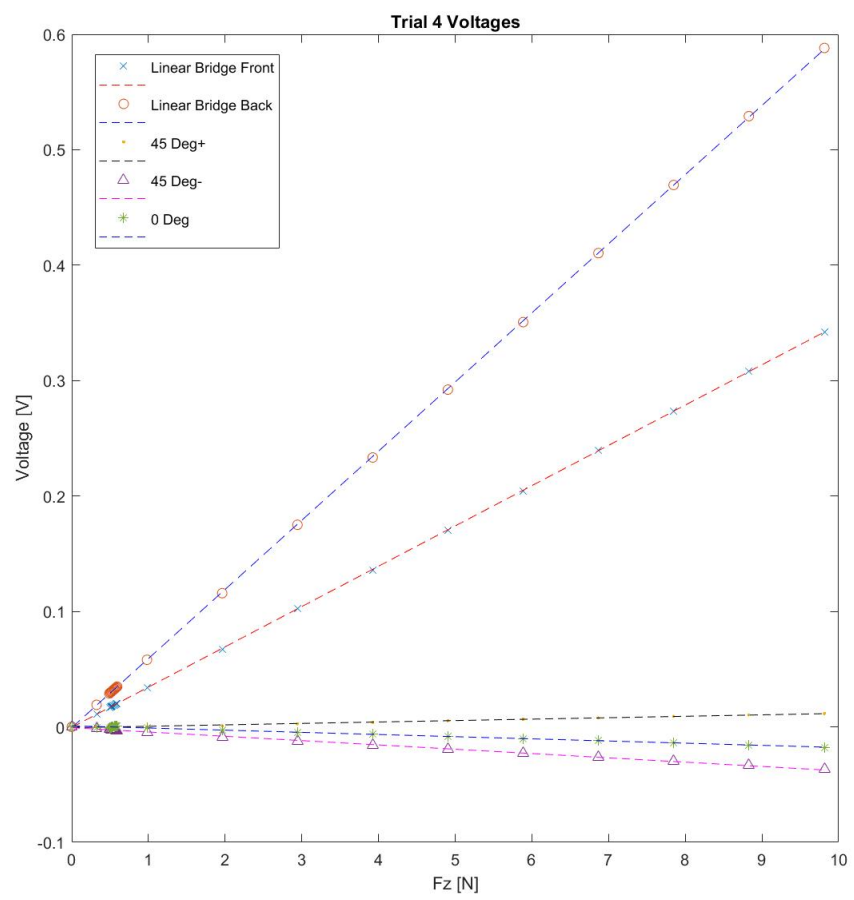

(d) Trial 4

Figure G.2: Voltage Response of All Bridge Outputs for Each Trial of an $F_{z}$ Applied Load at Loading Position 1 


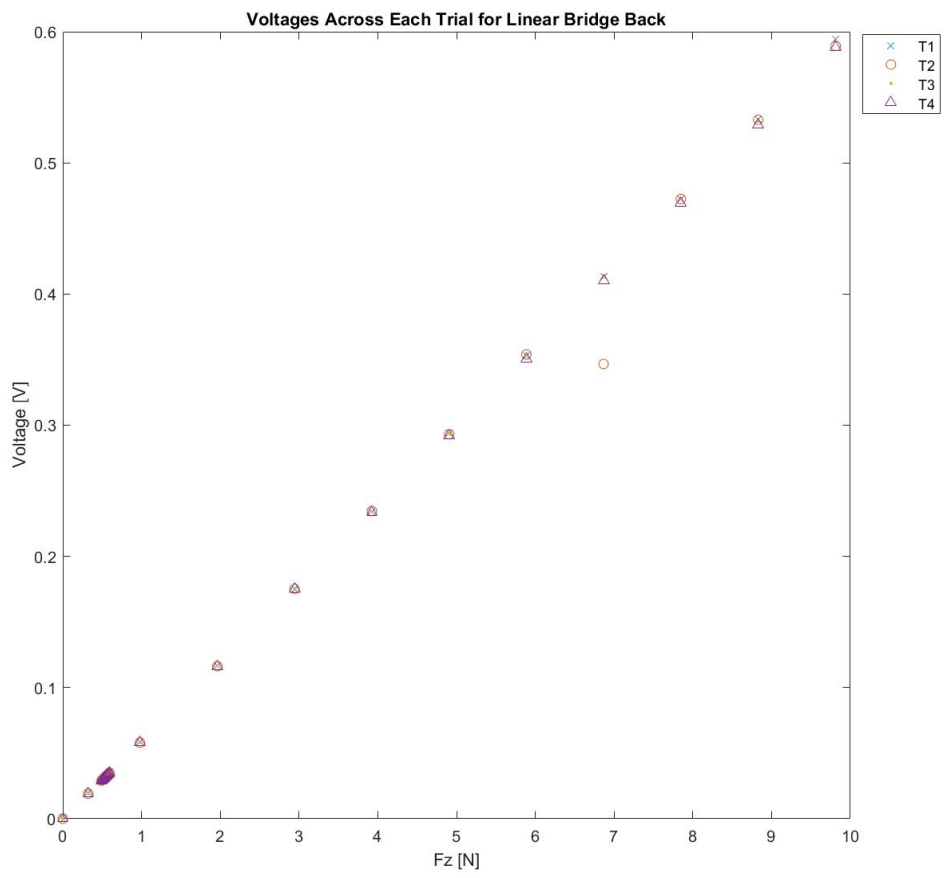

(a) Plane A

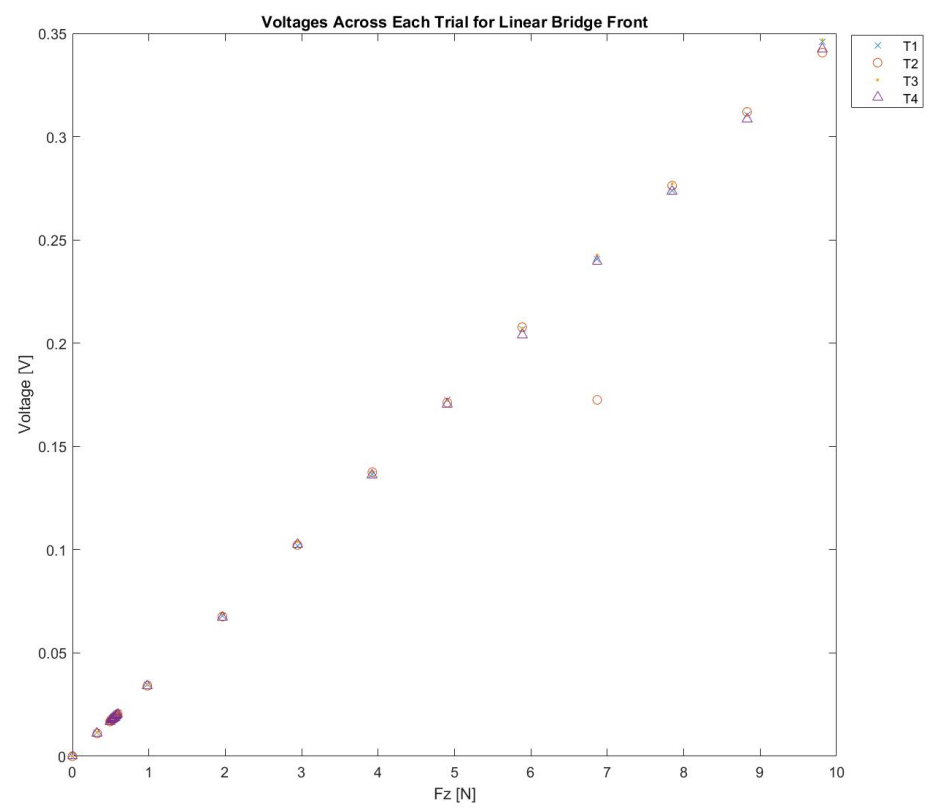

(b) Plane B

Figure G.3: Voltage Response of the Repeat Trials at Position 1 for the Linear Gauge Pairs, for an $F_{z}$ Applied Load 


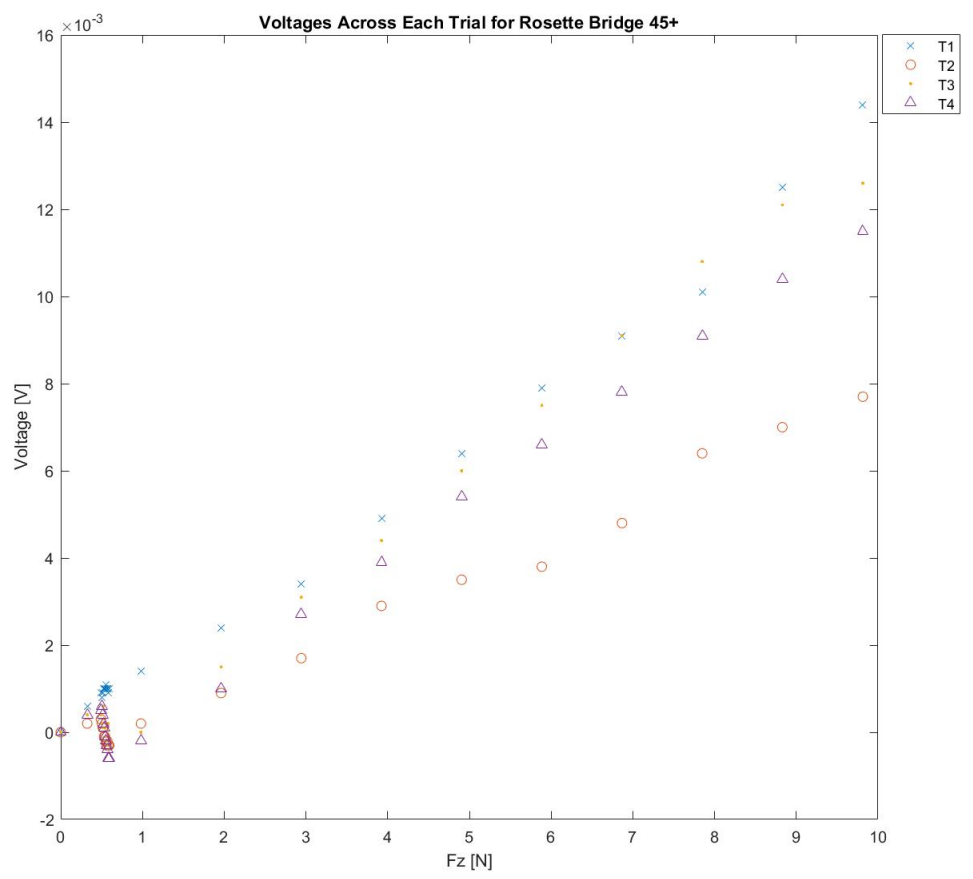

(a) Rosette $45^{\circ}(+)$ Response

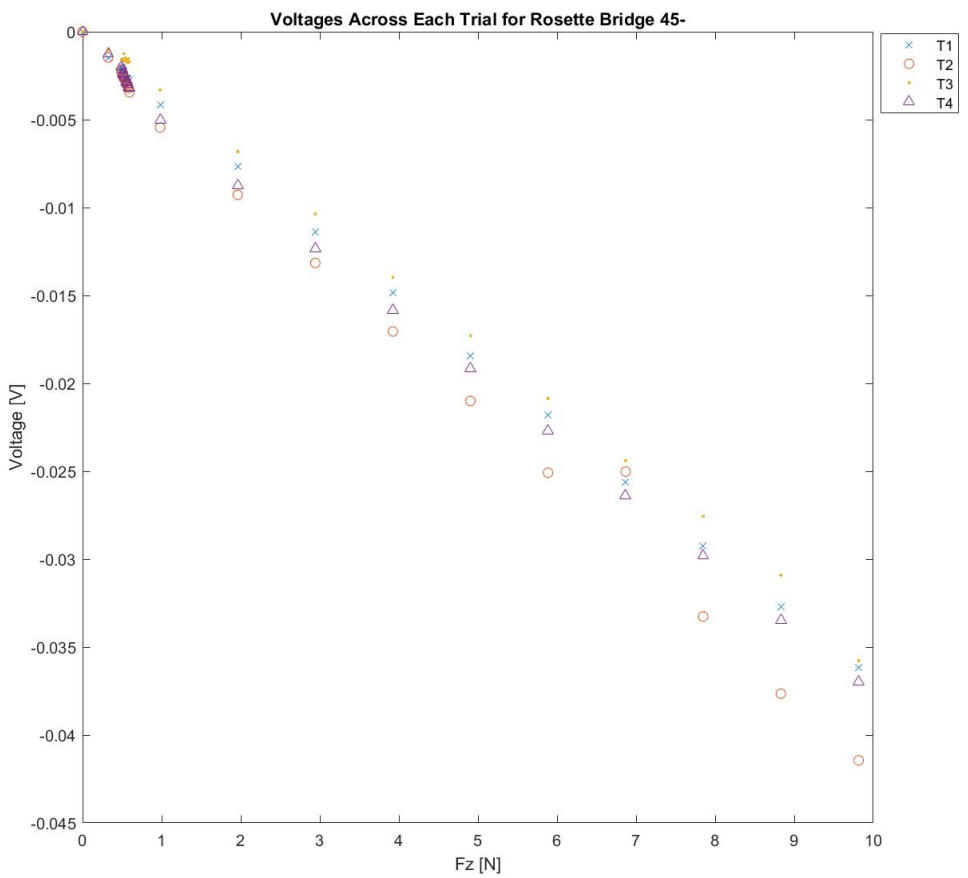

(b) Rosette $45^{\circ}$ (-) Response 


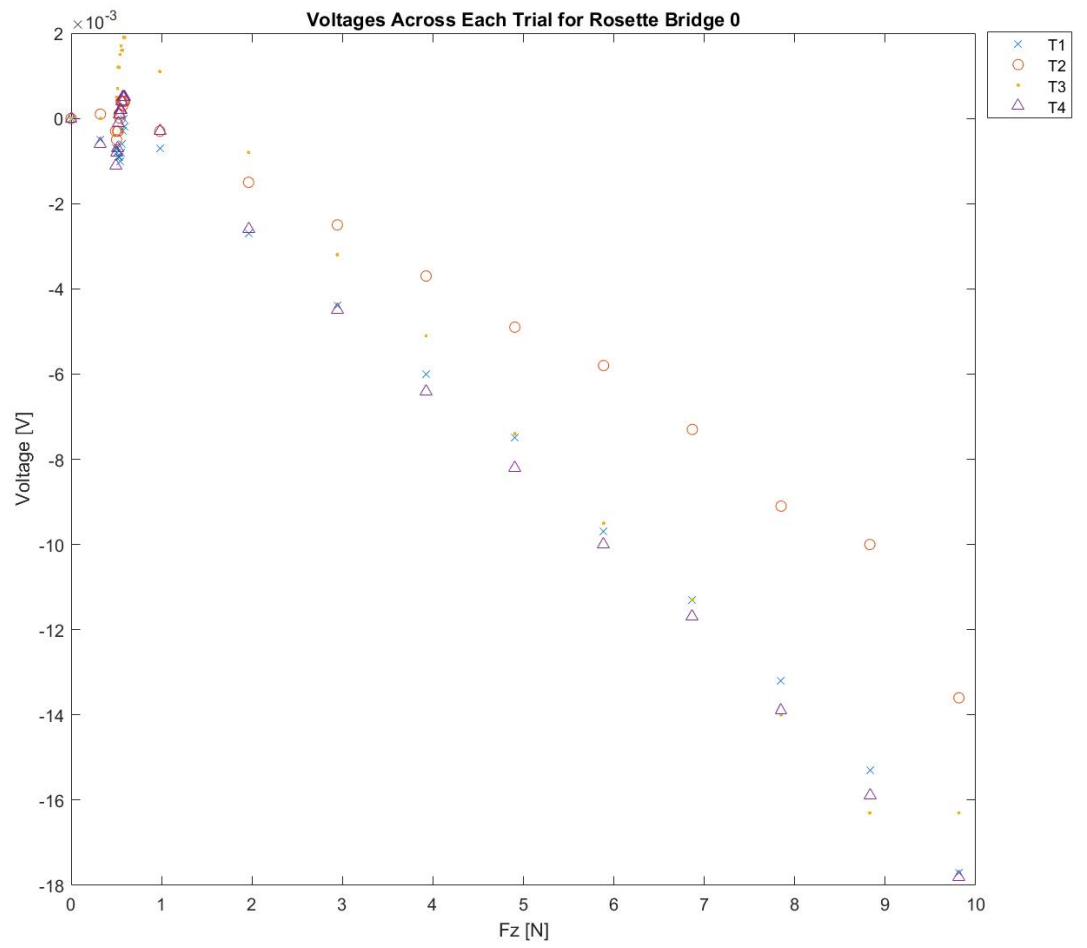

(c) Rosette $0^{\circ}$ Response

Figure G.4: Voltage Response of the Repeat Trials at Position 1 for the Rosettes, for an $F_{z}$ Applied Load 


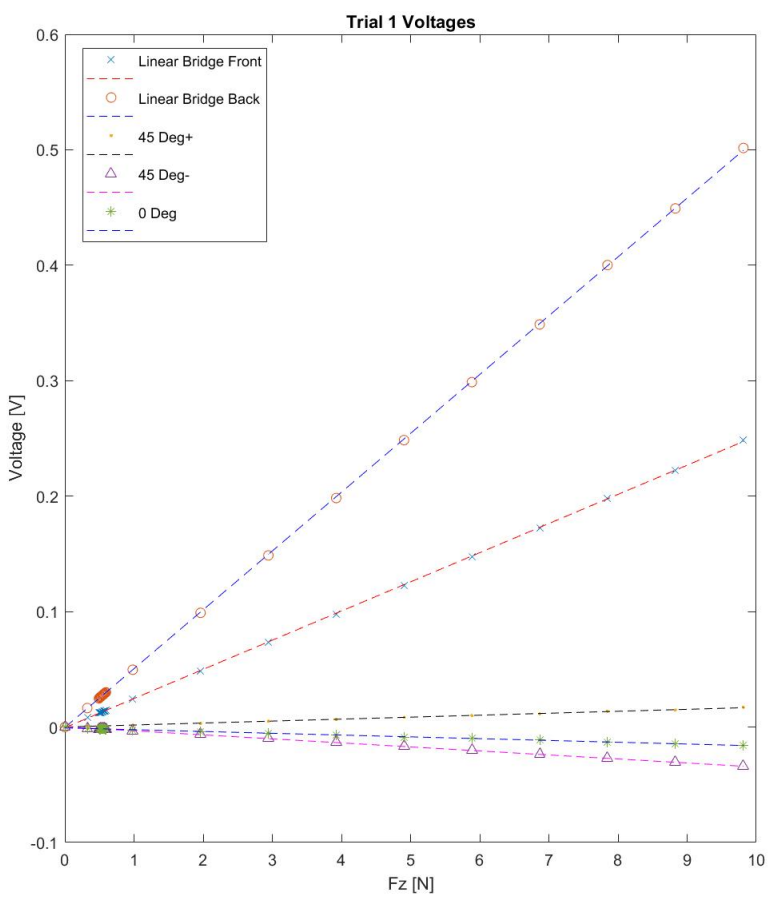

(a) Trial 1

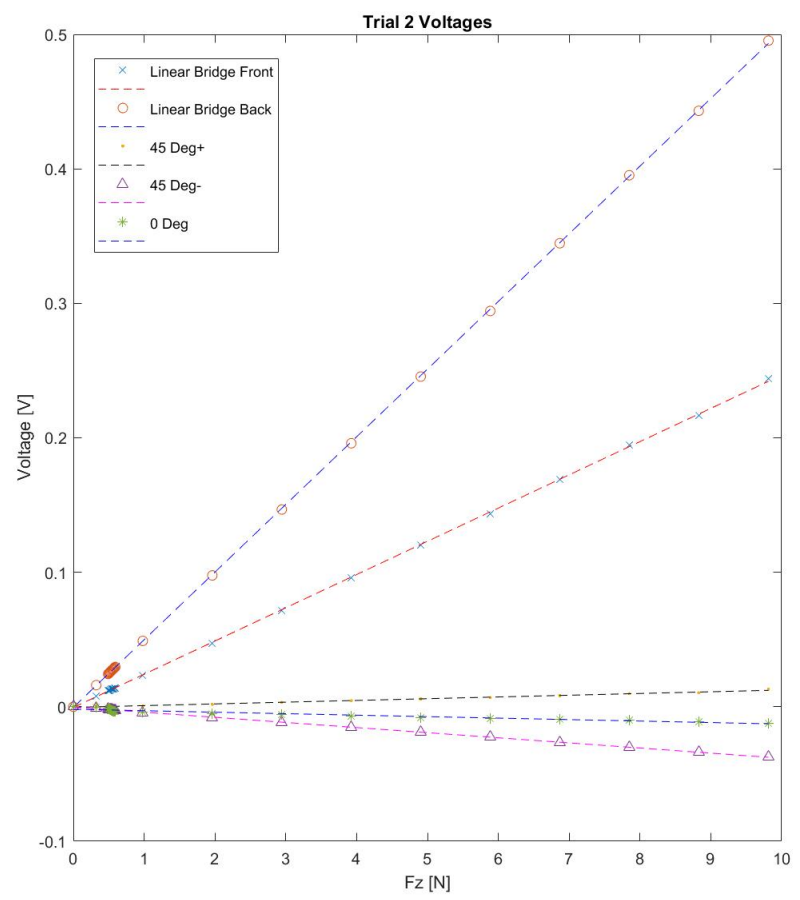

(b) Trial 2 


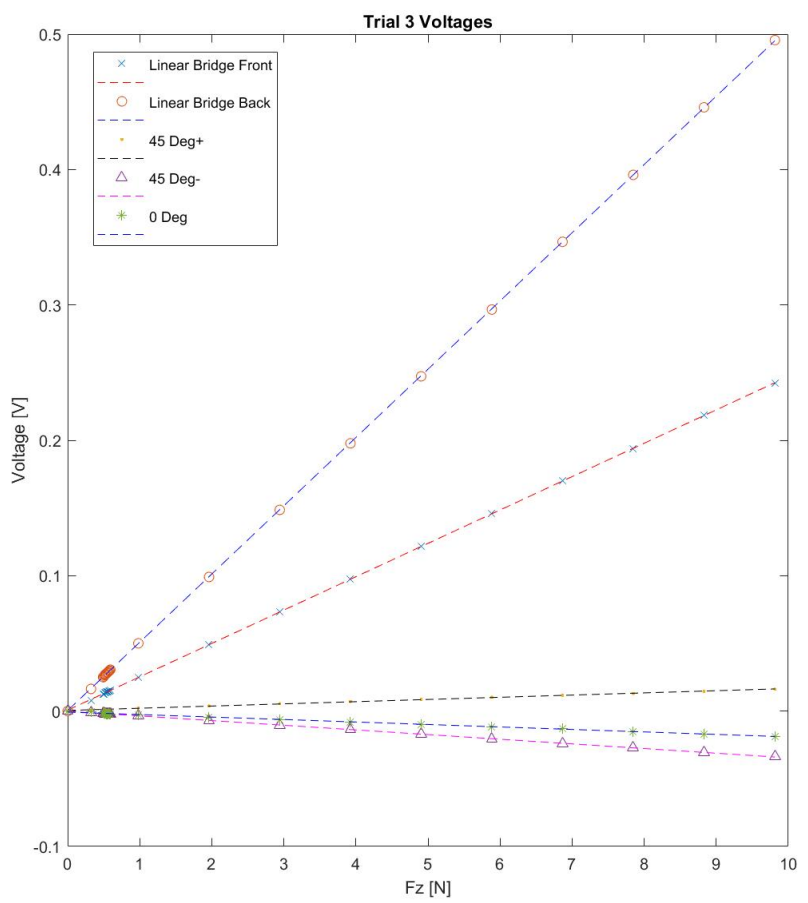

(c) Trial 3

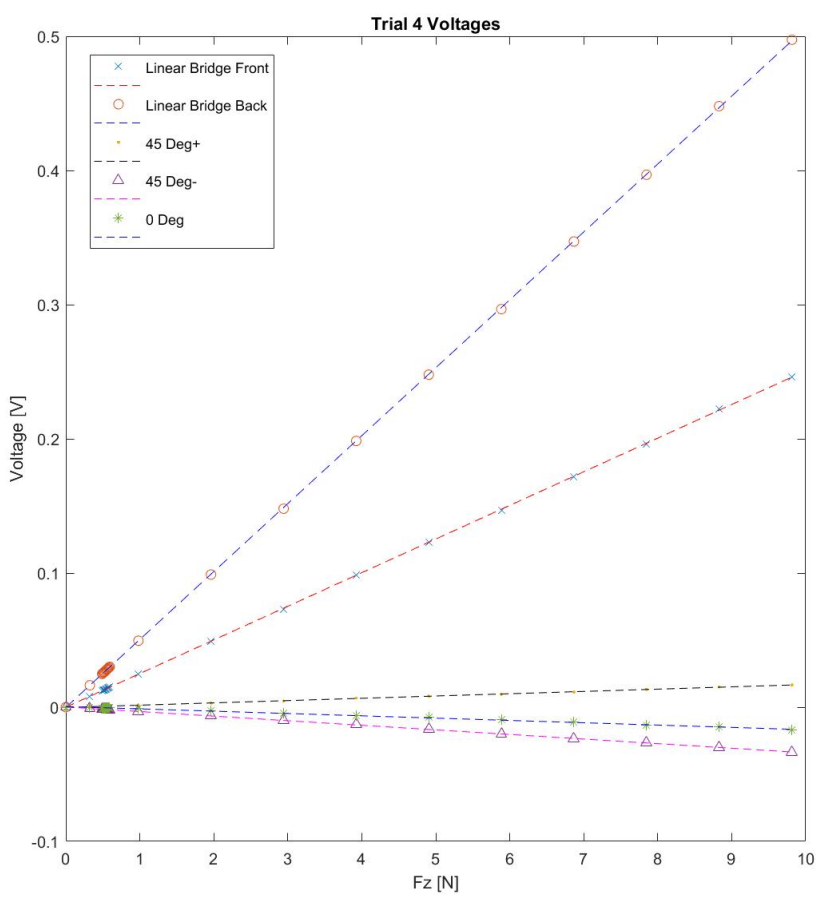

(d) Trial 4

Figure G.5: Voltage Response of All Bridge Outputs for Each Trial of an $F_{z}$ Applied Load at Loading Position 8 


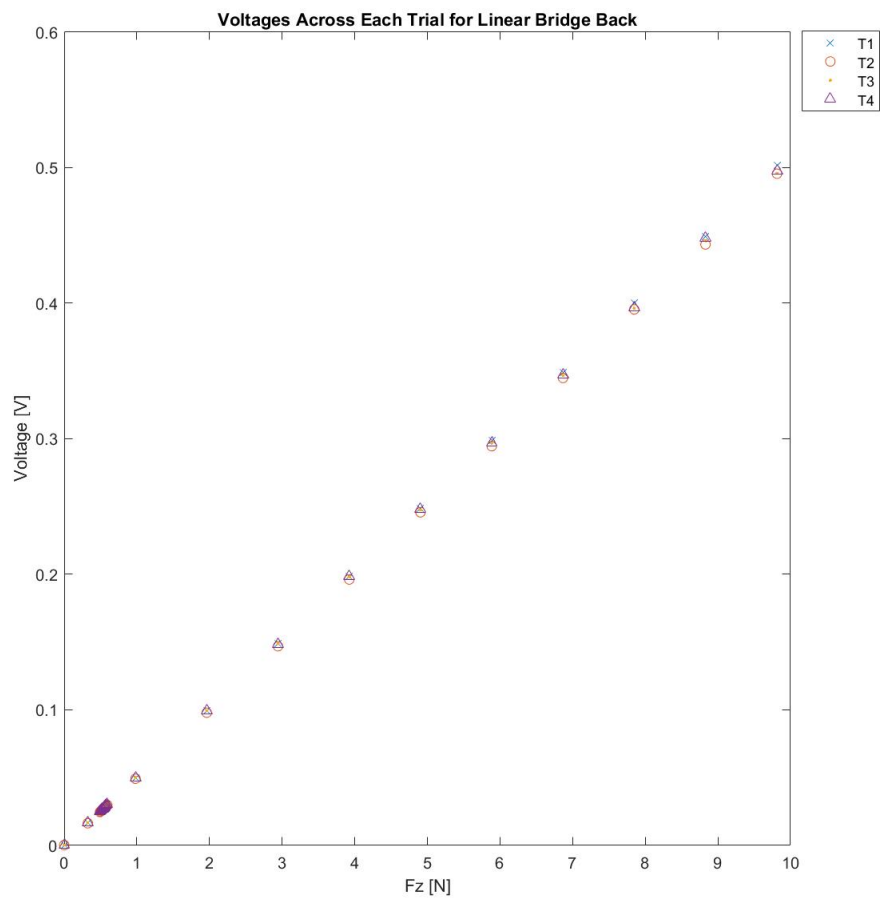

(a) Plane A

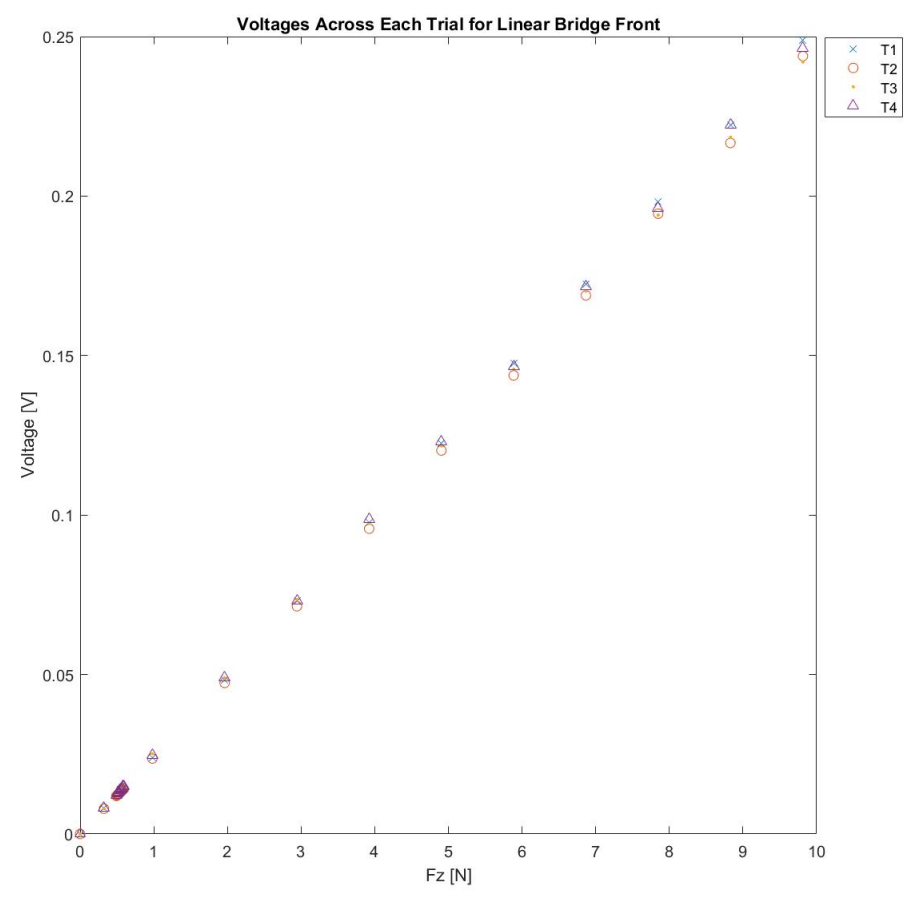

(b) Plane B

Figure G.6: Voltage Responses of Repeated Trials at Position 8 for the Linear Gauge Pairs, for an $F_{z}$ Applied Load 


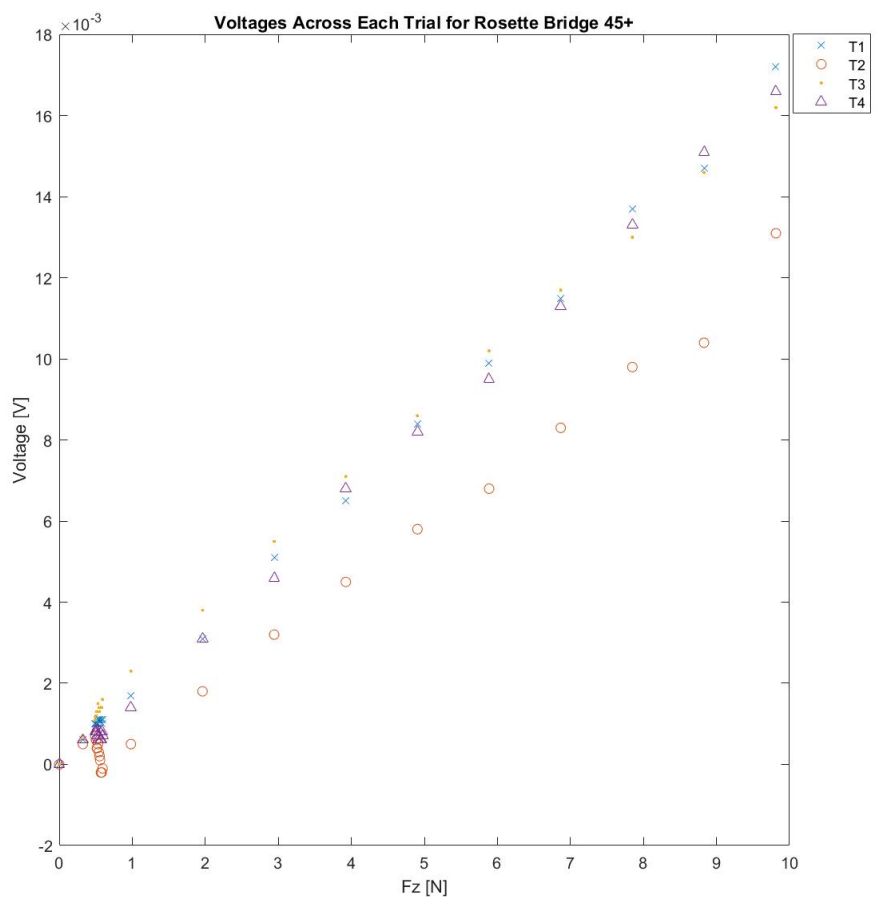

(a) Rosette $45^{\circ}(+)$ Response

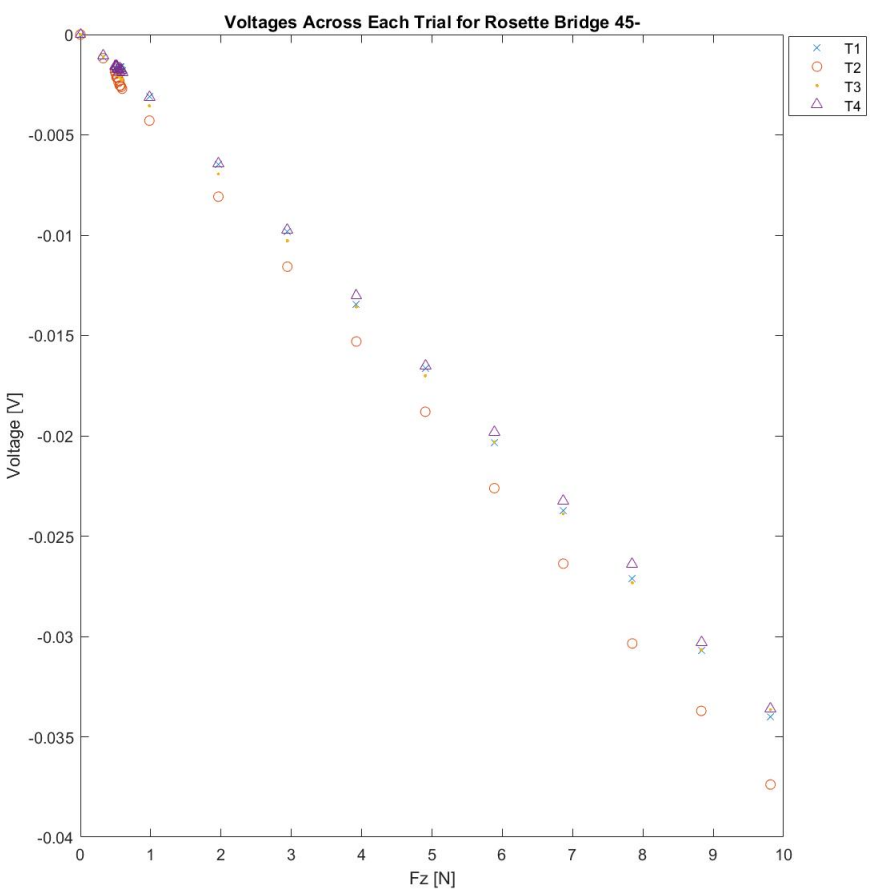

(b) Rosette $45^{\circ}$ (-) Response 


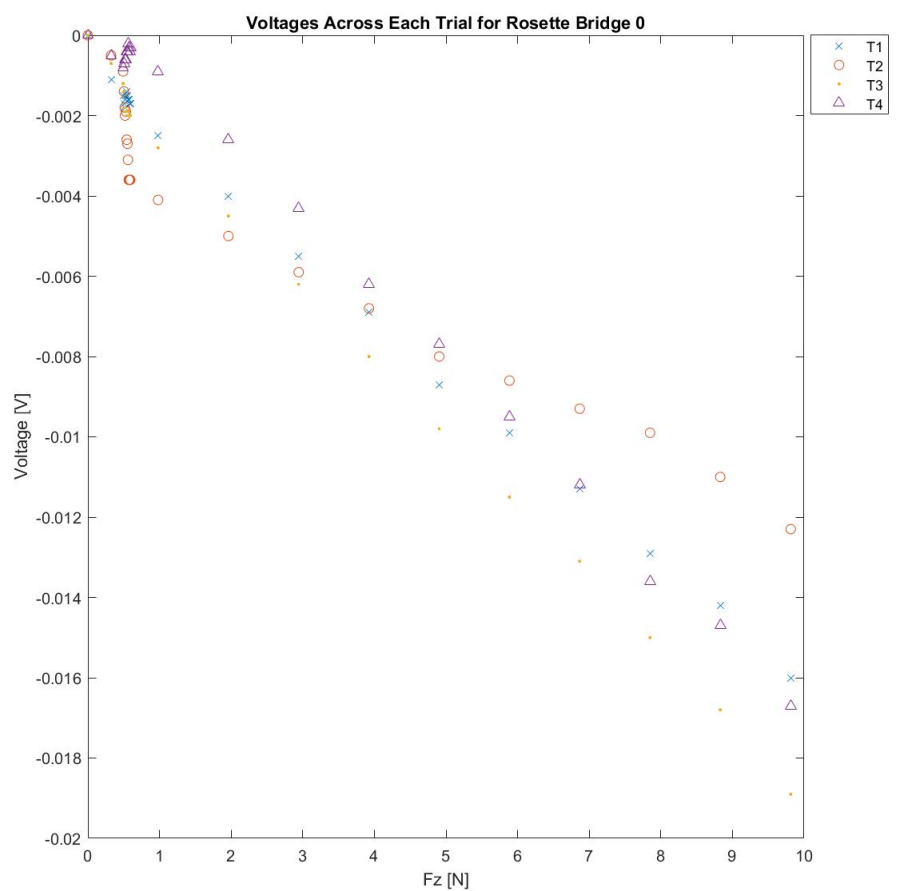

(c) Rosette $0^{\circ}$ Response

Figure G.7: Voltage Response of the Repeated Trials at Position 8 for the Rosettes, for an $F_{z}$ Applied Load 


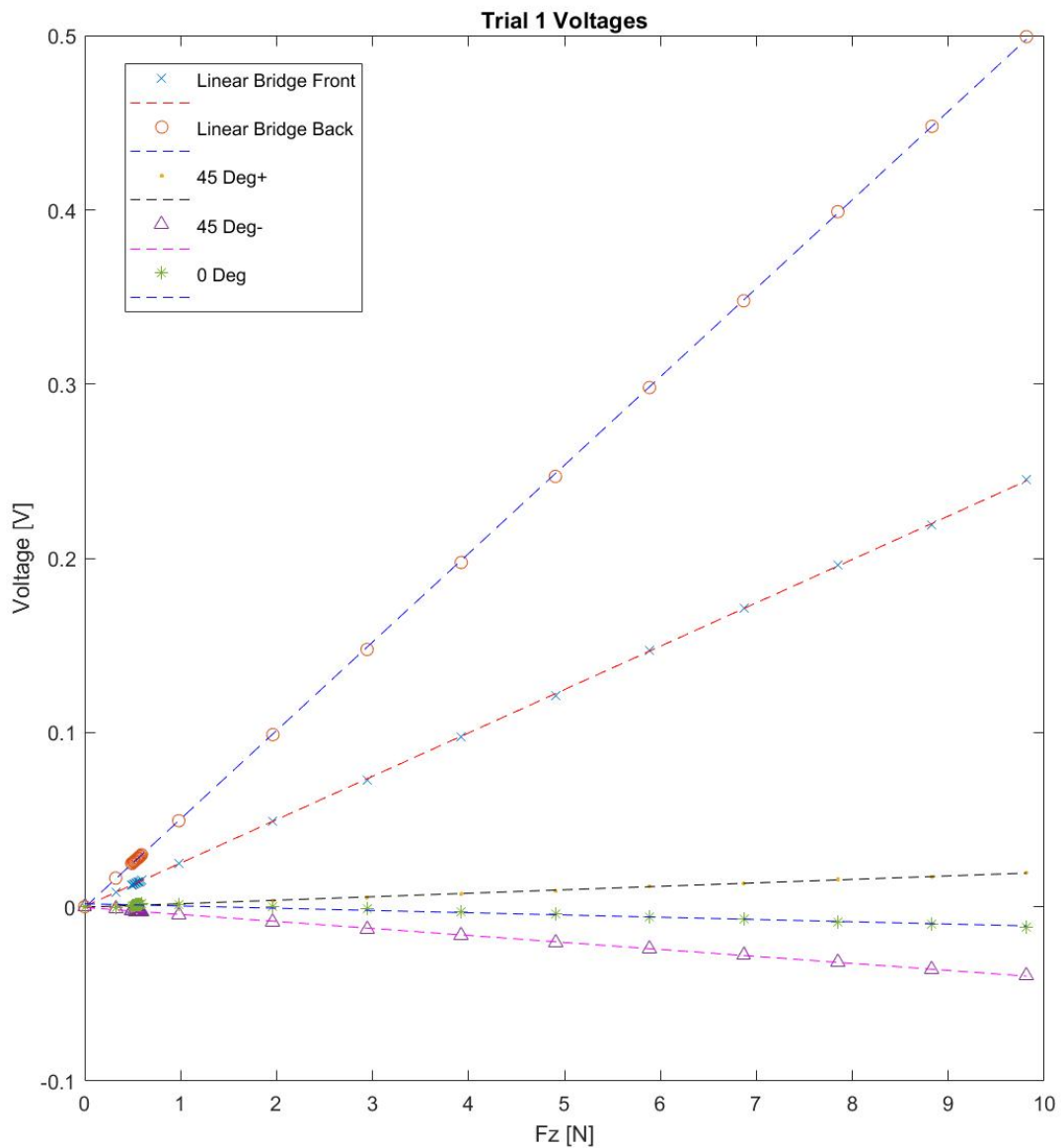

Figure G.8: Voltage Responses of All Bridge Outputs at Position 3, Trial 1 for $F_{z}$ 


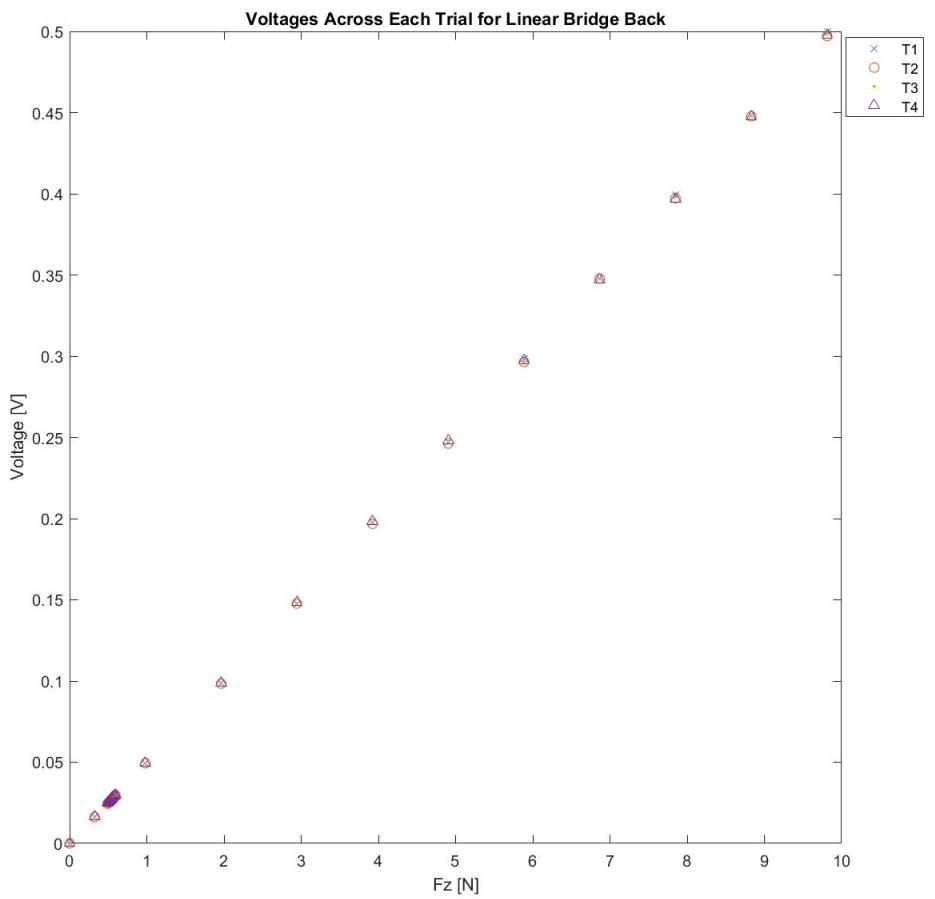

(a) Plane A

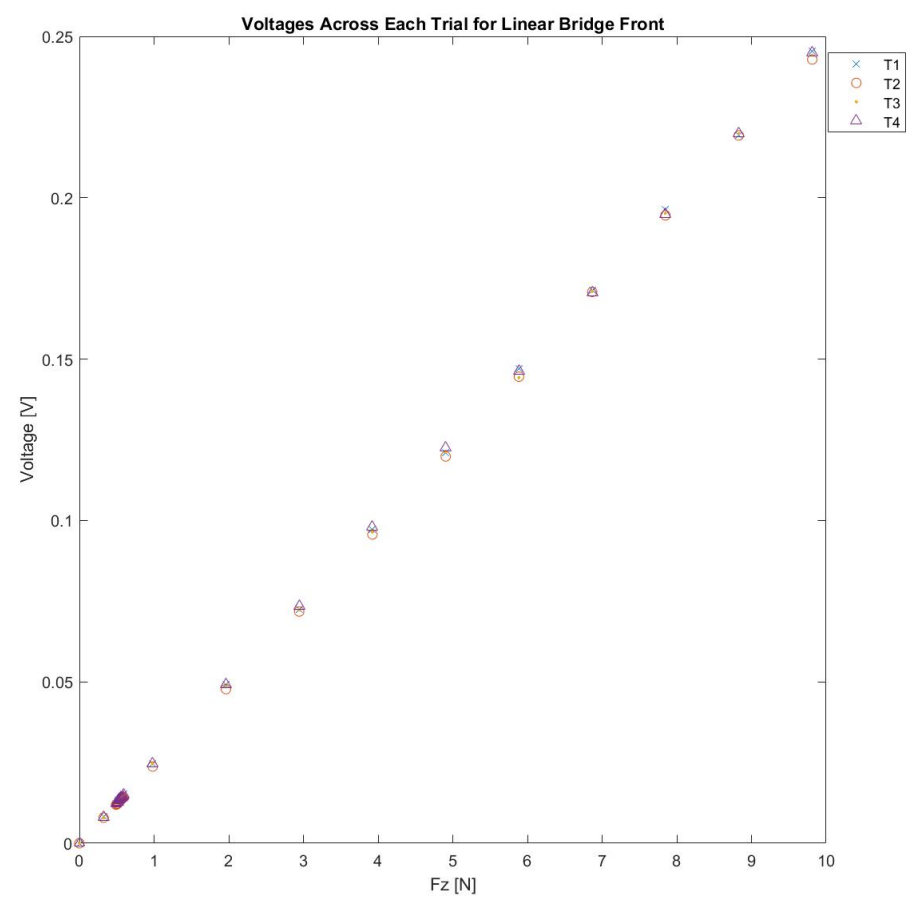

(b) Plane B

Figure G.9: Voltage Responses of Repeated Trials at Position 3 for the Linear Gauge Pairs, for an $F_{z}$ Applied Load 


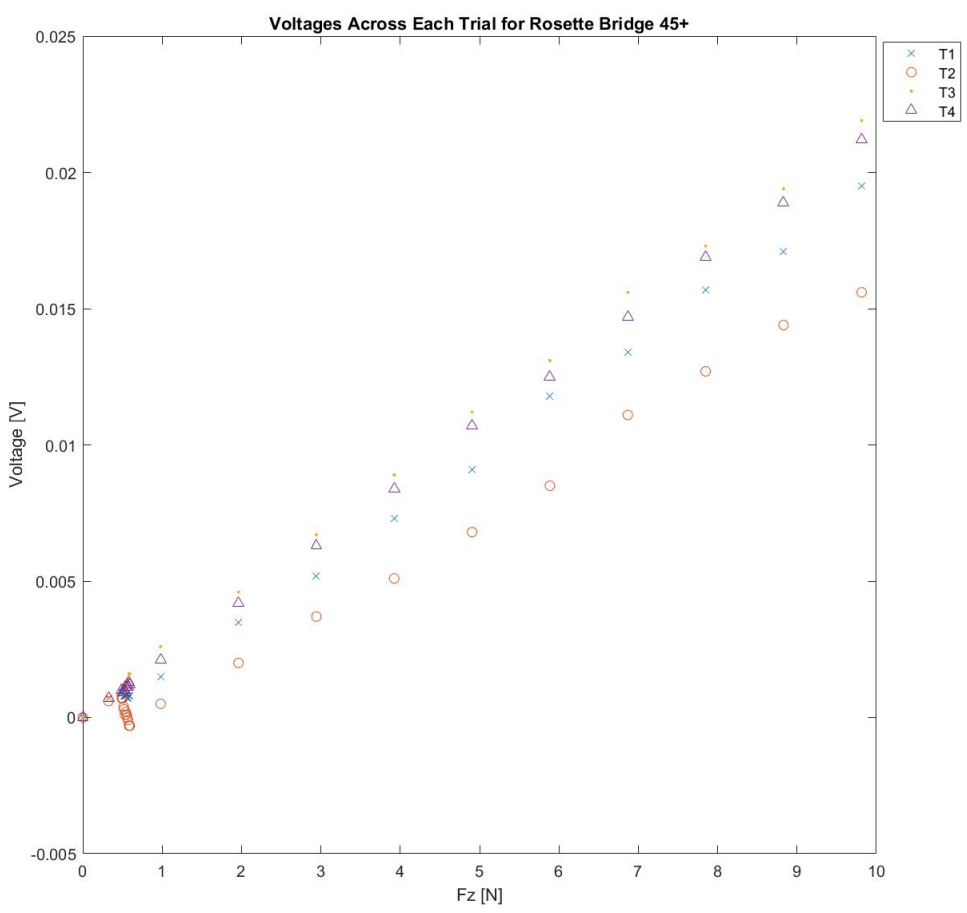

(a) Rosette $45^{\circ}(+)$ Response

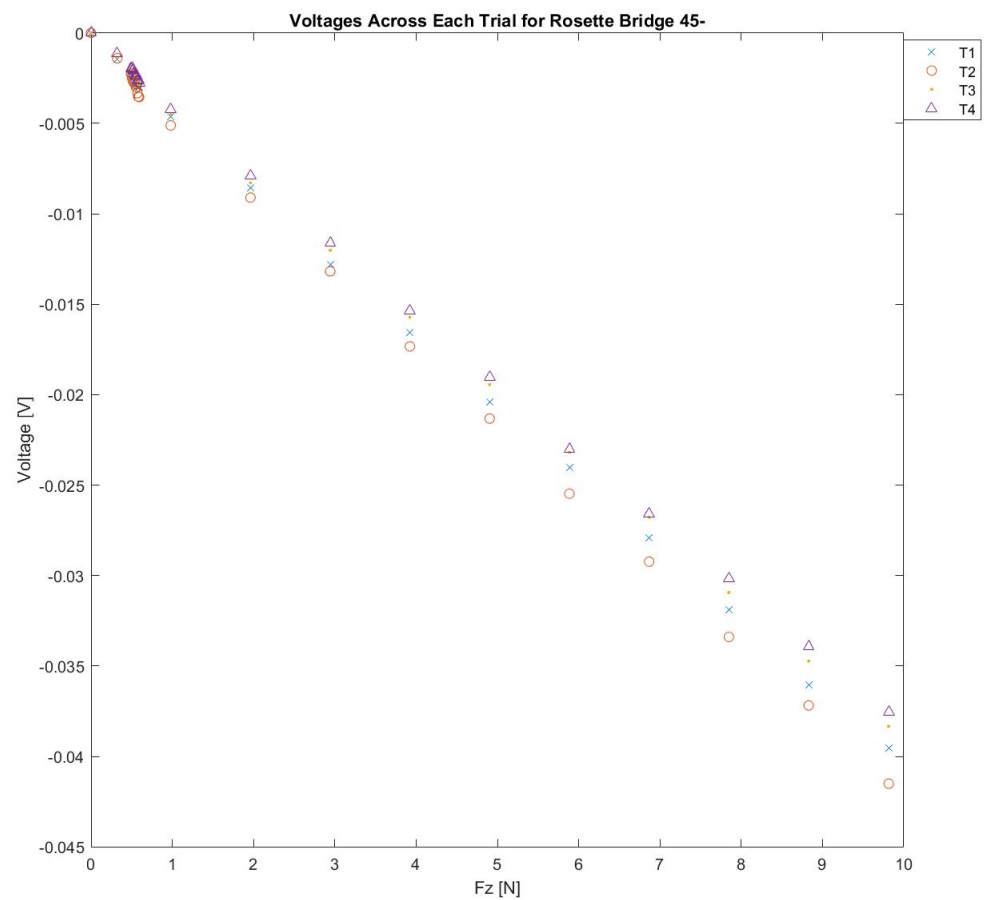

(b) Rosette $45^{\circ}$ (-) Response 


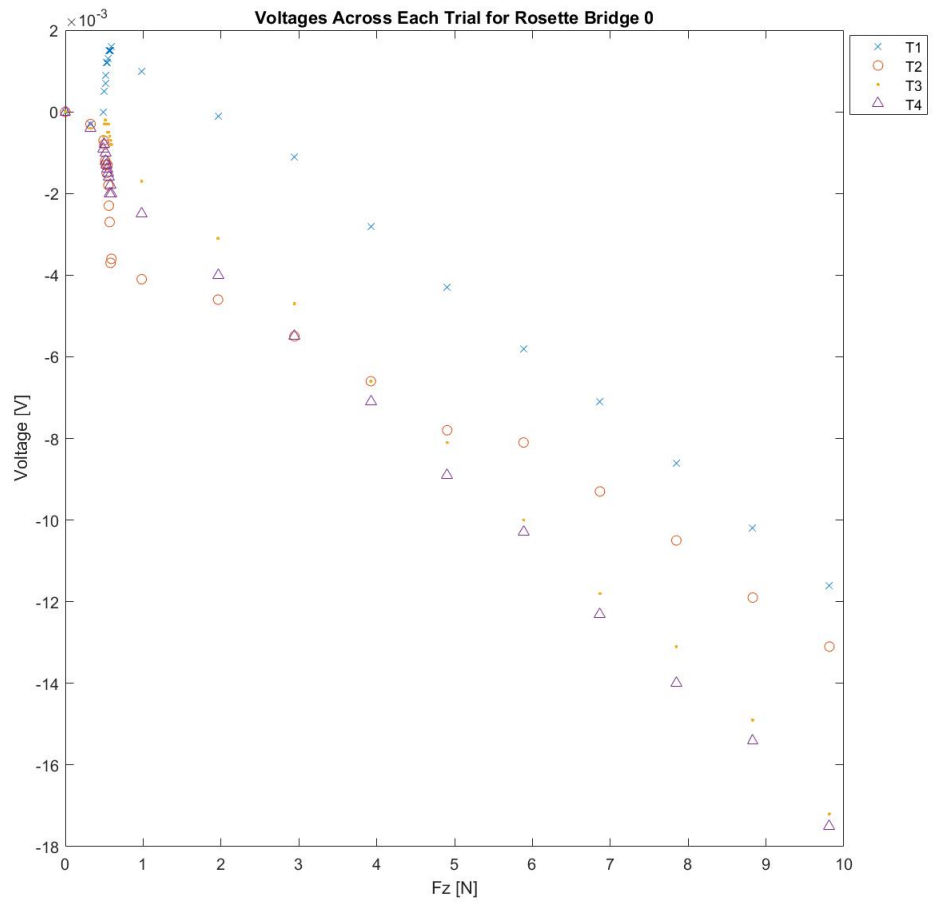

(c) Rosette $0^{\circ}$ Response

Figure G.10: Voltage Response of the Repeated Trials at Position 3 for the Rosettes, for an $F_{z}$ Applied Load 


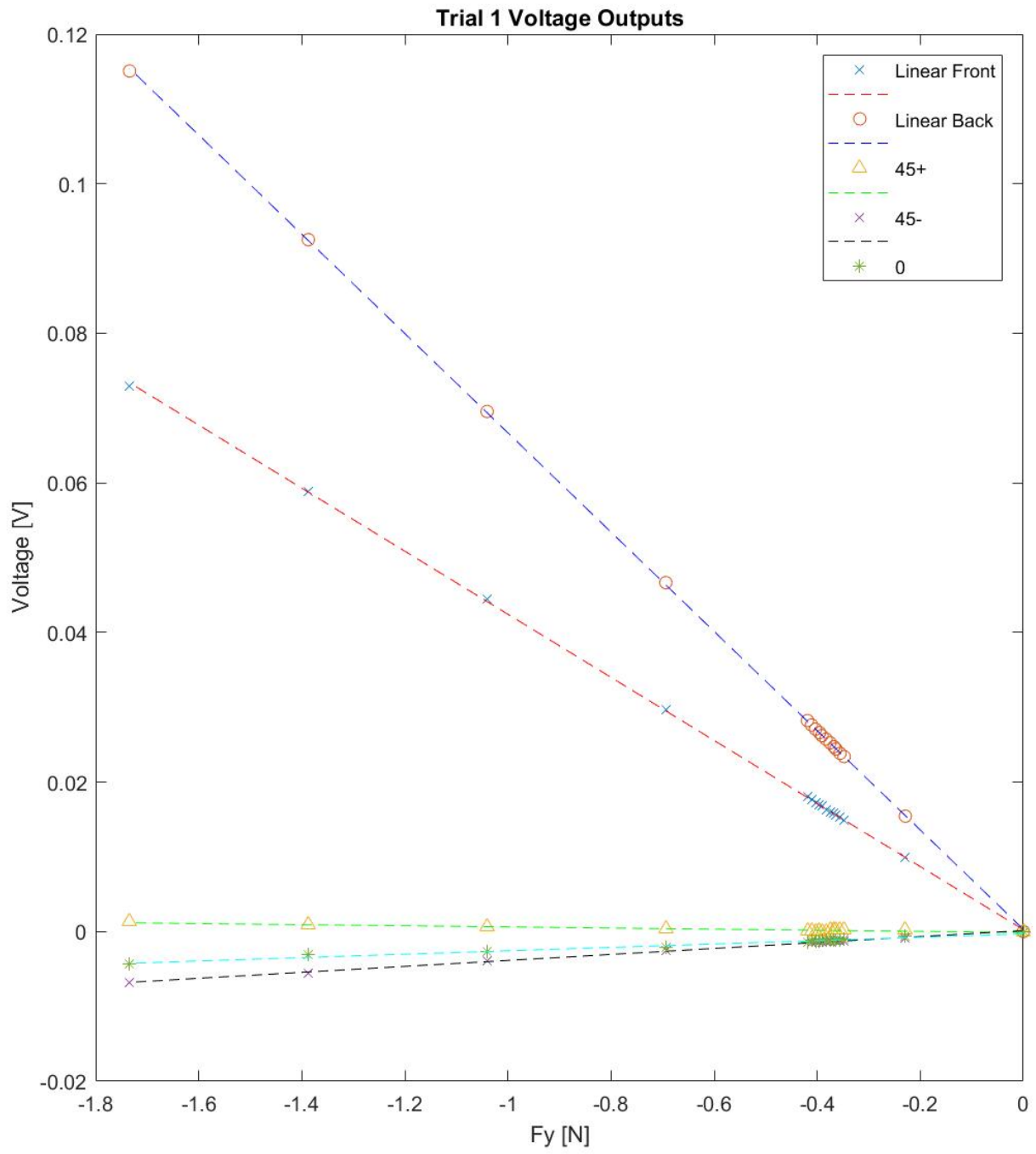

Figure G.11: Voltage Responses of All Bridge Outputs at Orientation 3, Trial 1 for the $F_{y}$ Testing 


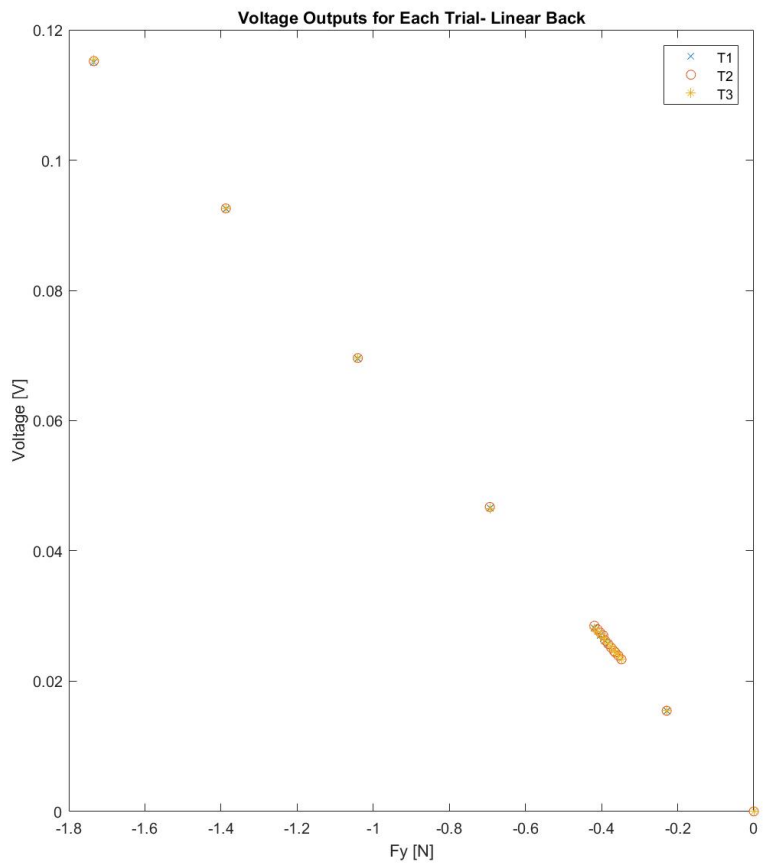

(a) Plane A

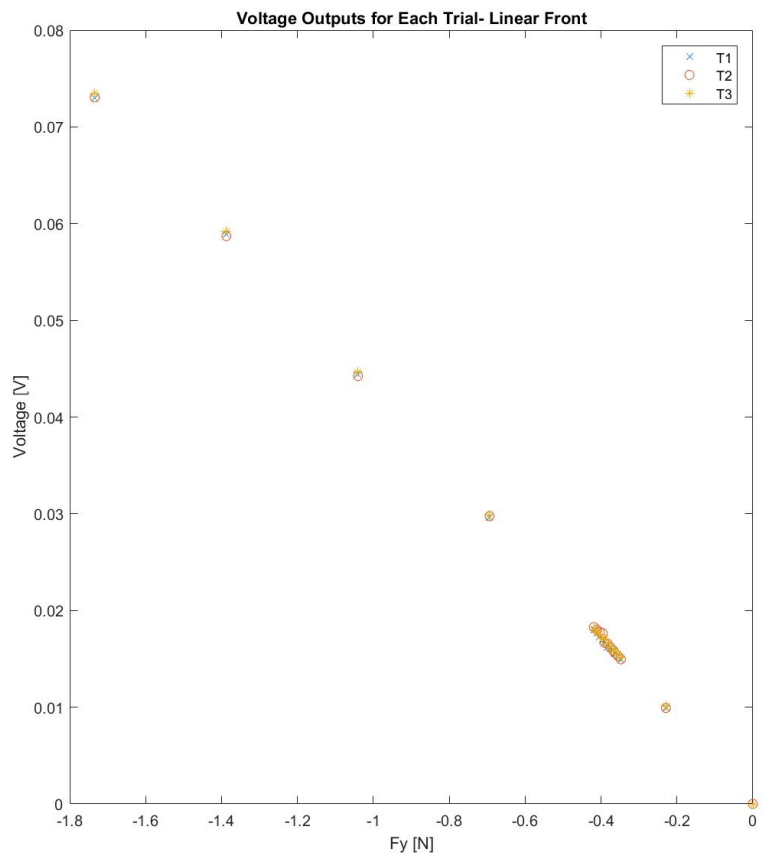

(b) Plane B

Figure G.12: Voltage Response of Linear Gauge Pairs from the Repeated Trials at Orientation 3 for the $F_{y}$ Testing 


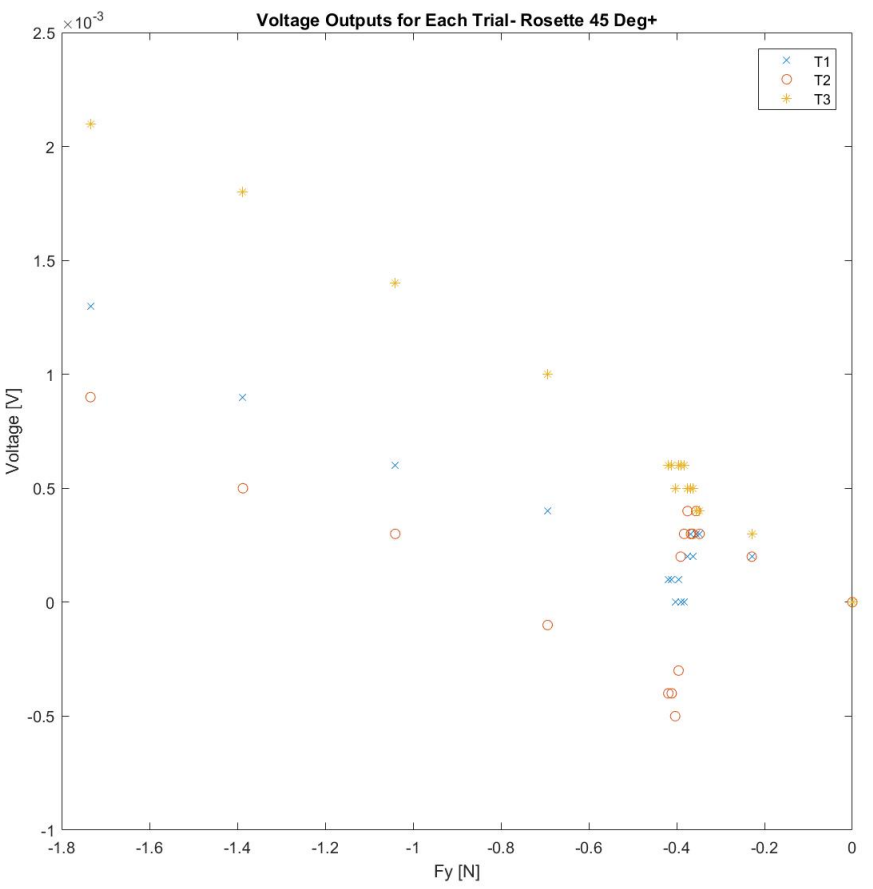

(a) Rosette $45^{\circ}(+)$ Response

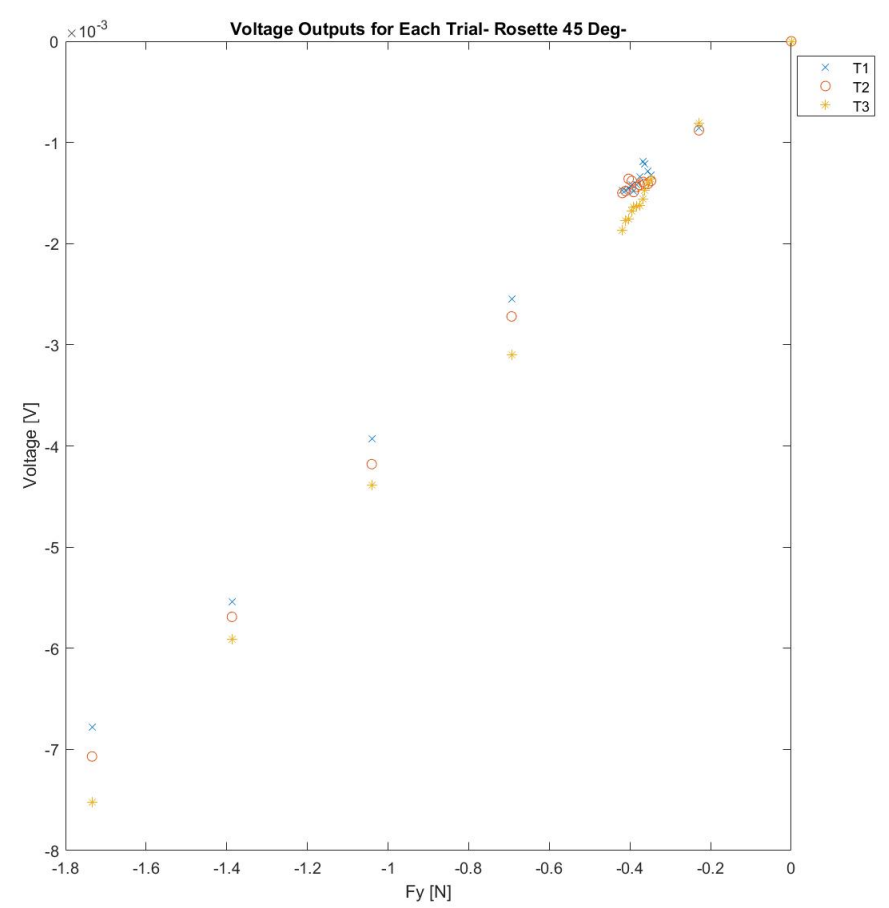

(b) Rosette $45^{\circ}$ (-) Response 


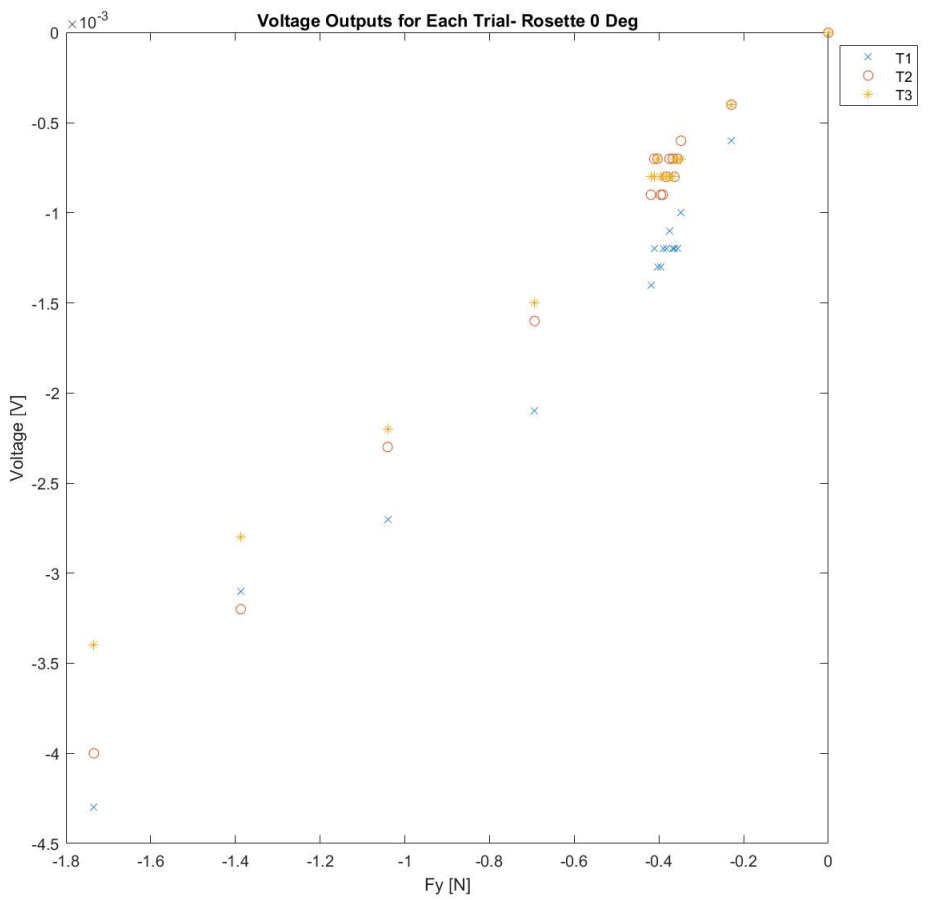

(c) Rosette $0^{\circ}$ Response

Figure G.13: Voltage Response of Rosettes from the Repeated Trials at Orientation 3 for the $F_{y}$ Testing 


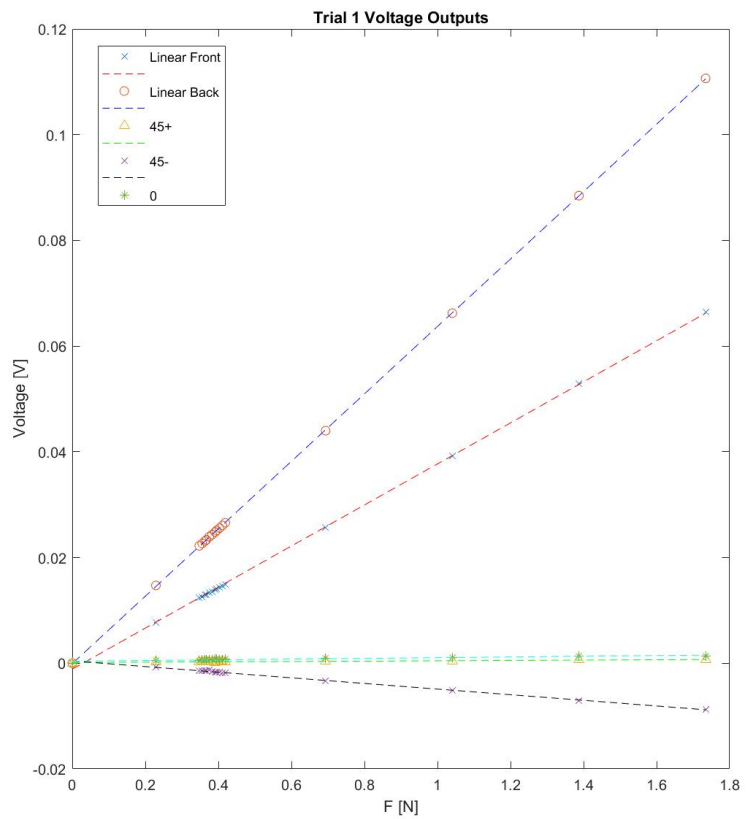

(a) Trial 1

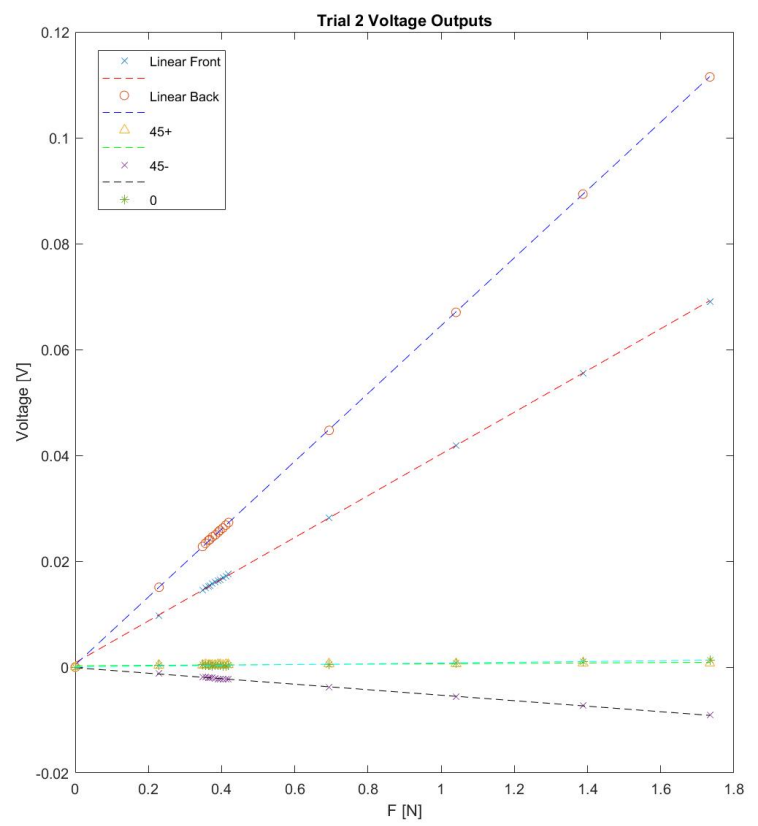

(b) Trial 2 


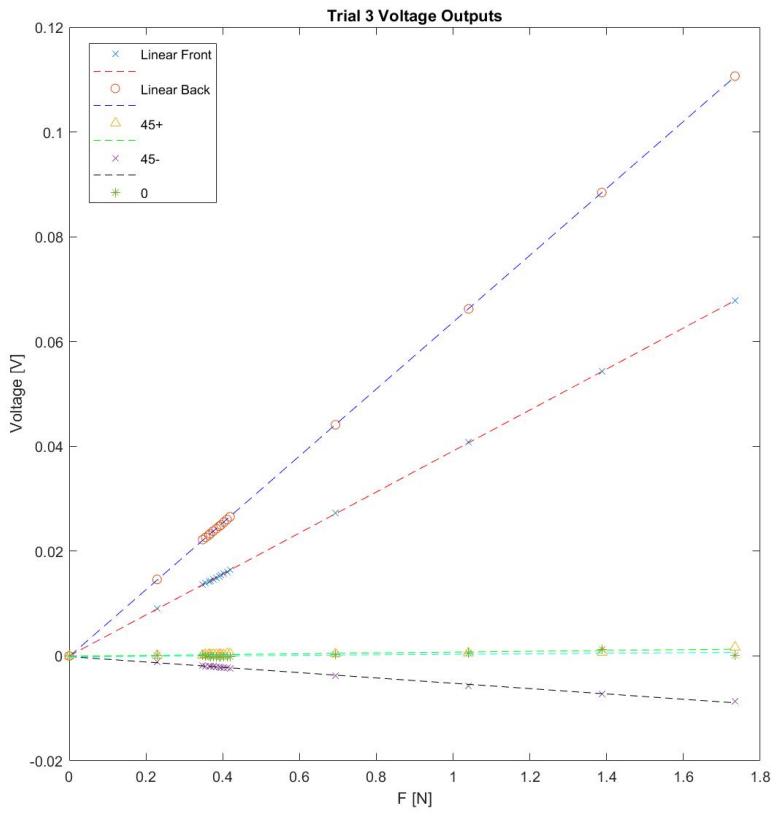

(c) Trial 3

Figure G.14: Voltage Response of All Bridge Outputs for Each Trial of an $F_{y}$ Testing at Orientation 5 


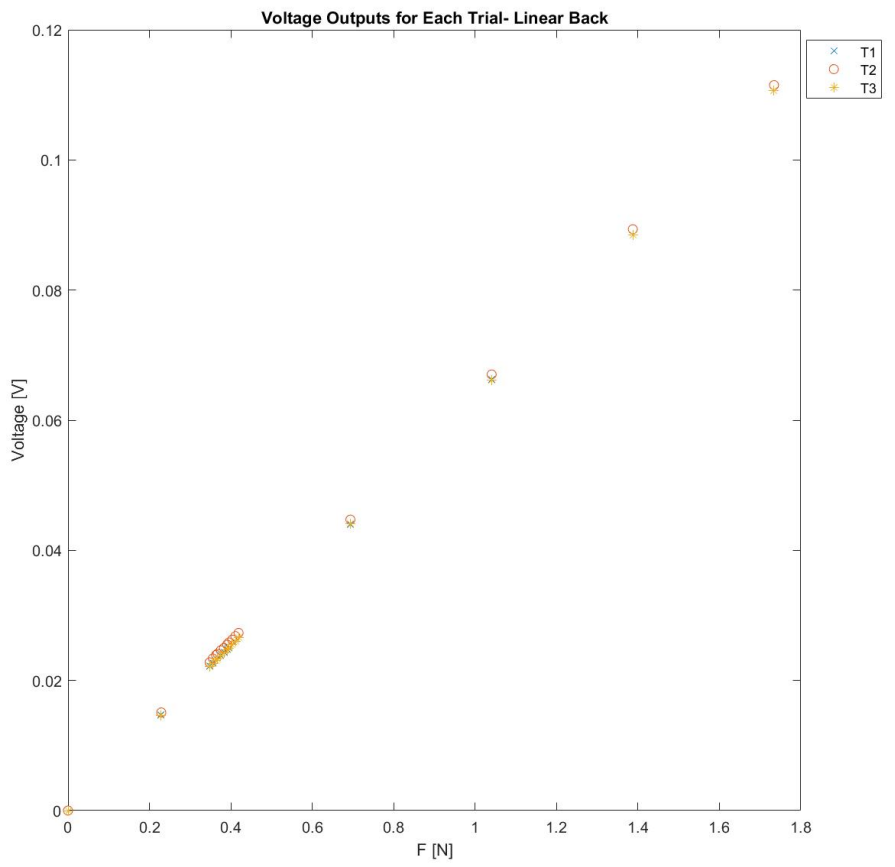

(a) Plane A

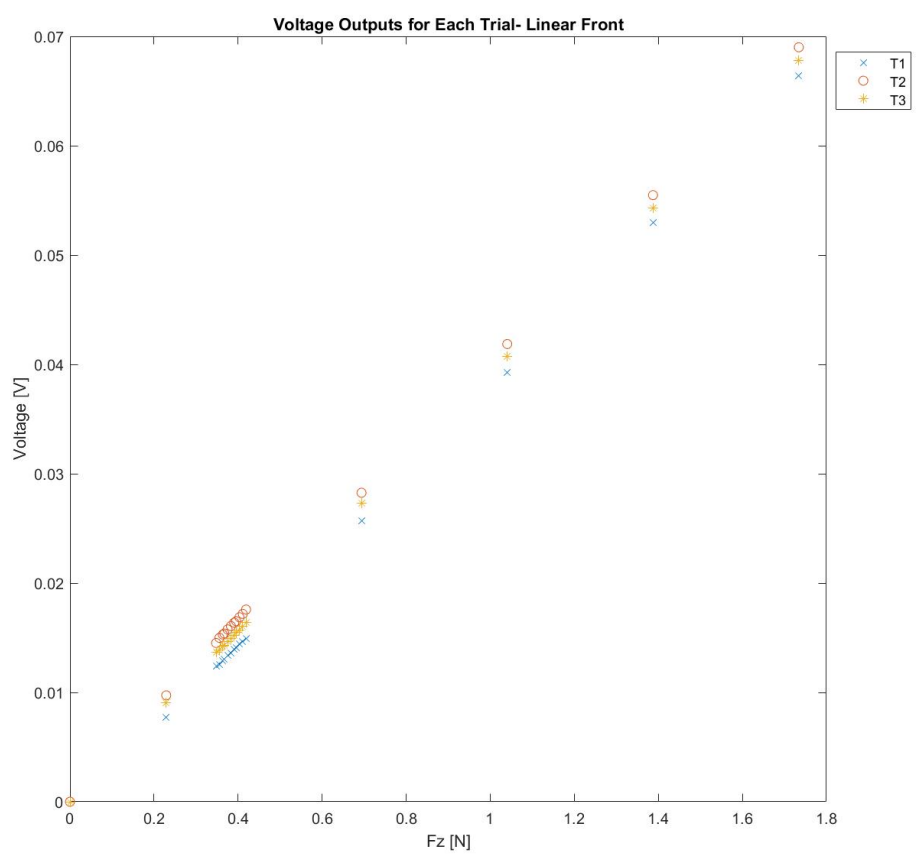

(b) Plane B

Figure G.15: Voltage Response of Linear Gauge Pairs from the Repeated Trials at Orientation 5 for the $F_{y}$ Testing 


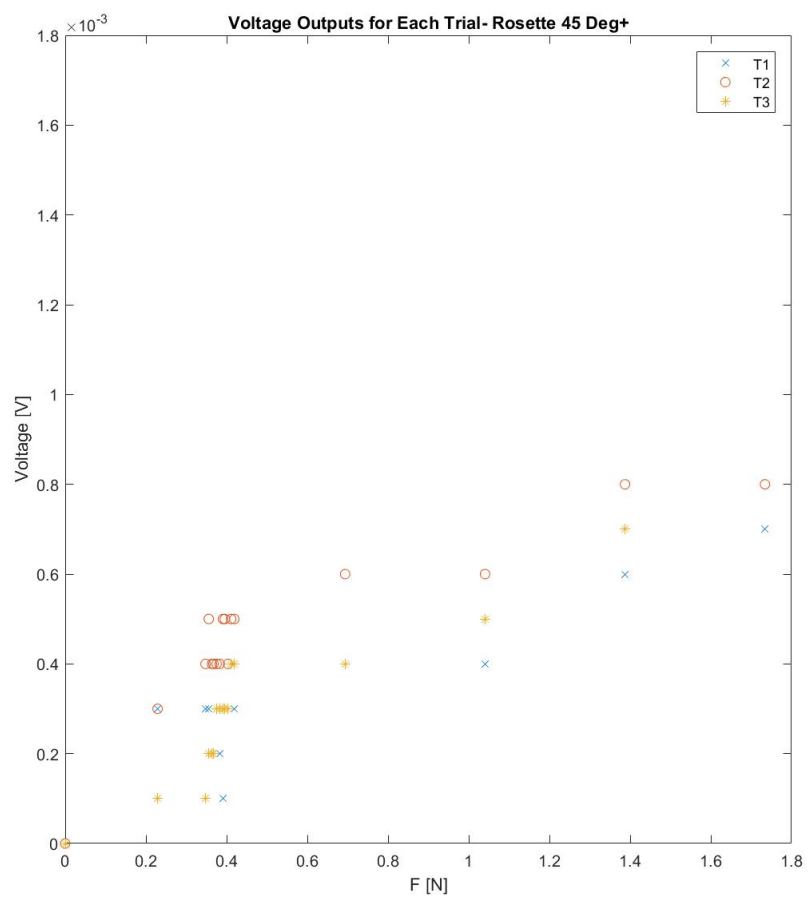

(a) Rosette $45^{\circ}(+)$ Response

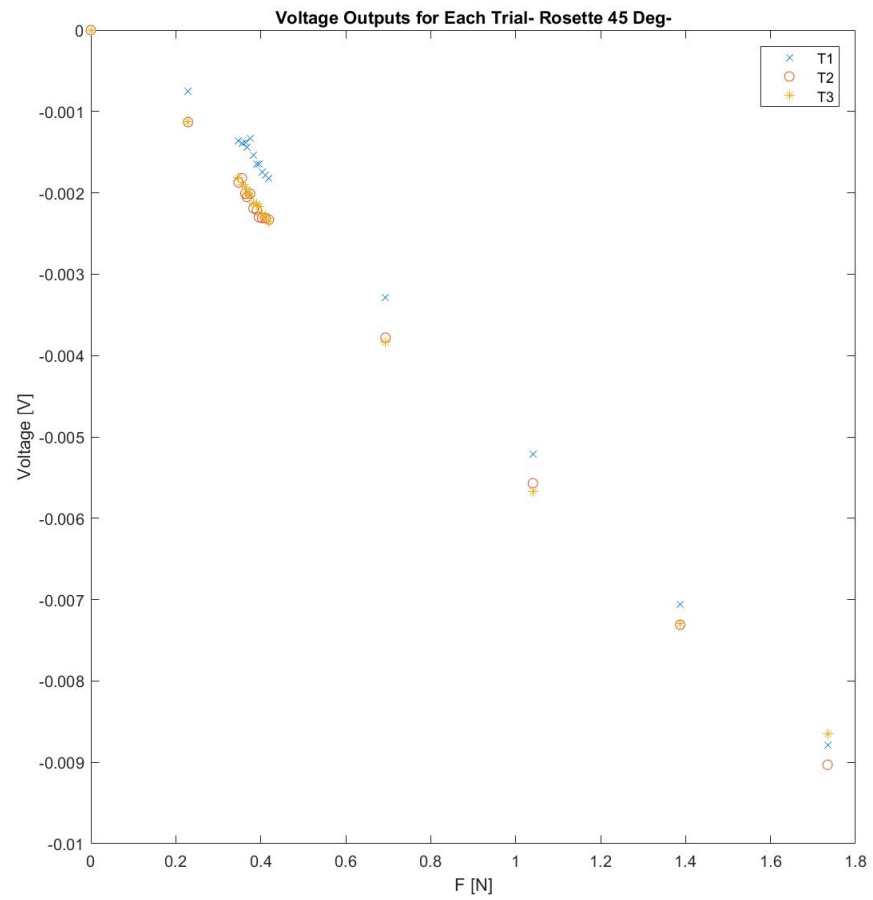

(b) Rosette $45^{\circ}$ (-) Response 


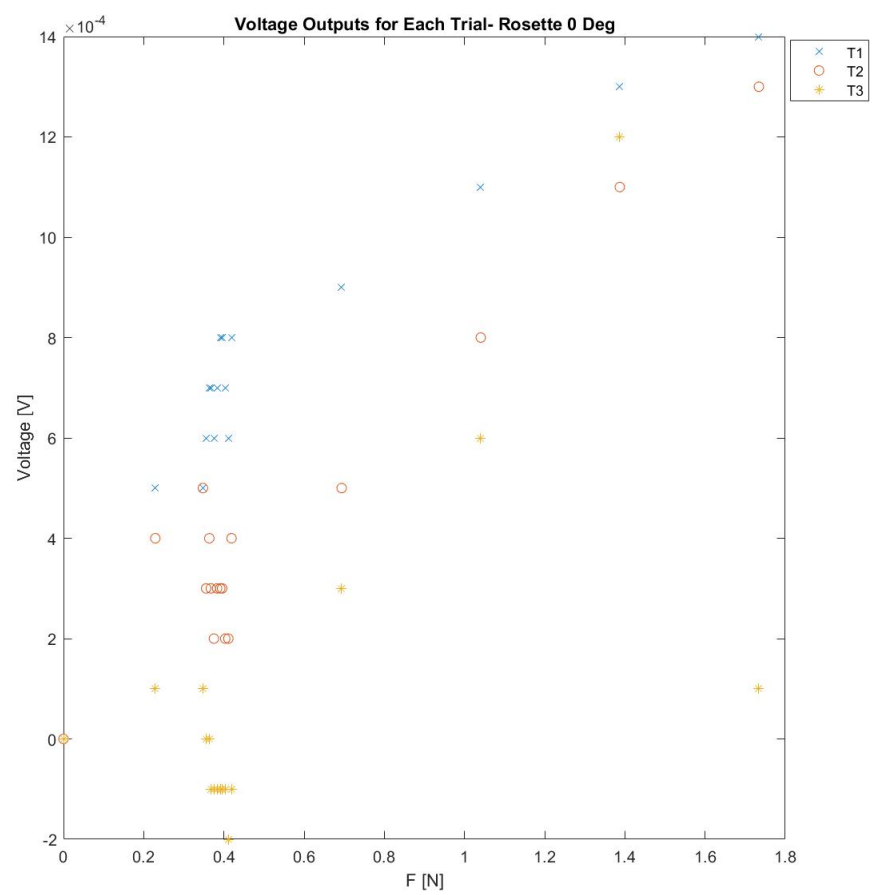

(c) Rosette $0^{\circ}$ Response

Figure G.16: Voltage Response of Rosettes from the Repeated Trials at Orientation 5 for the $F_{y}$ Testing 


\section{Appendix $\mathbf{H}$}

\section{Code Listing: Experimental Validation Data Collection}

The following appendix provides the Matlab code which was used to collect the voltage readings and user input values for each calibration test. The main script was developed from the LJM Matlab library from LabJack, and edited to meet the data collection requirements.

$\%$ script edited from LJM Matlab library from LabJack

$\%$ ensure thesis folder and subfolders are added to the path,...

\%allowing MATLAB library to be accessible

close all

clear all

\%Make connected LabJack visible

ljmAsm = NET $\cdot \operatorname{addAssembly}($ 'LabJack.LJM');

\%Creating an object to nested class LabJack.LJM.CONSTANTS

$\mathrm{t}=1 \mathrm{jmAsm}$. AssemblyHandle.GetType ('LabJack.LJM+CONSTANTS') ;

LJM_CONSTANTS $=$ System.Activator. CreateInstance $(t)$;

handle $=0$; 
$\%$ Test Inputs

\% \% \% \% \% \% \% \% \% \% \% \% \% \% \% \% \% \% \% \% \% \% \% \% \% \% \% \% \% \% \% \% \% \% \% \% \% \% \% \% \% \% \% \% \% \% \% \% \% \% \% \% \% \% \%

MassApplied $=0 ; \%[\mathrm{~kg}] \quad \%$ Input Value for Specific Testing Case

ForceMagnitude $=$ MassApplied $* 9.81 ; \%[\mathrm{~N}]$

$\% x[-11-+11], \quad z[16.2]$ y $[0-156]$

xcontact $=0 / 1000 ; \quad \%[m] \quad \%$ Input Value for Specific Testing Case

ycontact $=0 / 1000 ; \quad \%[m] \%$ Input Value for Specific Testing Case

zcontact $=-10.175 / 1000 ; \%[\mathrm{~m}]$

theta_x $=90 ; \%$ [degrees, 0 is pure $\mathrm{x}$ ] \%Input Value for Specific Testing Case

theta_y $=90 ; \%$ [degrees, 0 is pure y] \%Input Value for Specific Testing Case

theta_z $=0 ; \%[$ degrees, 0 is pure z] \%Input Value for Specific Testing Case

$\%$ calculate the resultant force and moment due to the applied loading case

$\mathrm{Fx}=$ ForceMagnitude $* \cos d($ theta_x $) ; \%[N]$

Fy $=$ ForceMagnitude $* \cos d($ theta_y $) ; \%[N]$

$\mathrm{Fz}=$ ForceMagnitude $* \cos d($ theta_z $) ; \%[\mathrm{~N}]$

Mx_o = Fz*ycontact - Fy*zcontact;

My_o = Fx*zcontact - Fz*xcontact;

Mz_o = Fy*xcontact - Fx*ycontact;

$\mathrm{F}=\left[\begin{array}{llllll}\mathrm{Fx} & \mathrm{Fy} & \mathrm{Fz} & \mathrm{Mx} \mathrm{x}_{-} \mathrm{O} & \mathrm{My} \mathrm{O}_{-} \mathrm{O} & \mathrm{Mz} z_{-} \mathrm{O}\end{array}\right]^{\prime}$;

\% \% \% \% \% \% \% \% \% \% \% \% \% \% \% \% \% \% \% \% \% \% \% \% \% \% \% \% \% \% \% \% \% \% \% \% \% \% \% \% \% \% \% \% \% \% \% \% \% \% \% \% \% \% \% \% \%

try

\%Open T7 device, Any connection, Any identifier

[ljmError, handle] = LabJack.LJM.OpenS('T7', 'ANY', 'ANY', handle); showDeviceInfo(handle) 
$\%$ Stream Readings, this provides the number of channels to read and the $\%$ number of scans to complete, in this case over a 2 second window maxRequests $=2 ; \%$ the number of stream loops that will run scanRate $=$ double $(2000) ; \%$ Scans per second scansPerRead $=\operatorname{int32}($ scanRate $)$; numAINs $=12 ;$

firstAINChan $=0 ; \%$ Starting AIN channel. $0=$ AIN0. numAddresses $=$ numAINs;

$\%$ InAmp Information

$\%$ Scan list names to stream aScanListNames = NET.createArray ('System.String', numAddresses); for $i=1$ :numAddresses

chan $=$ firstAINChan $+i-1$ $\operatorname{aScanListNames}(i)=\left[{ }^{\prime A I N}\right.$ ' num2str(chan) $]$; end $\%$ Scan list addresses to stream aScanList $=$ NET.createArray ('System.Int32', numAddresses); \% Dummy array for aTypes parameter aTypes = NET.createArray ('System.Int32', numAddresses); LabJack.LJM.NamesToAddresses (numAddresses, aScanListNames, aScanList, aTypes);

$\%$ Stream reads will be stored in aData. Needs to be at least $\%$ numAddresses*scansPerRead in size. aData $=$ NET. createArray ('System.Double', numAddresses*scansPerRead); 


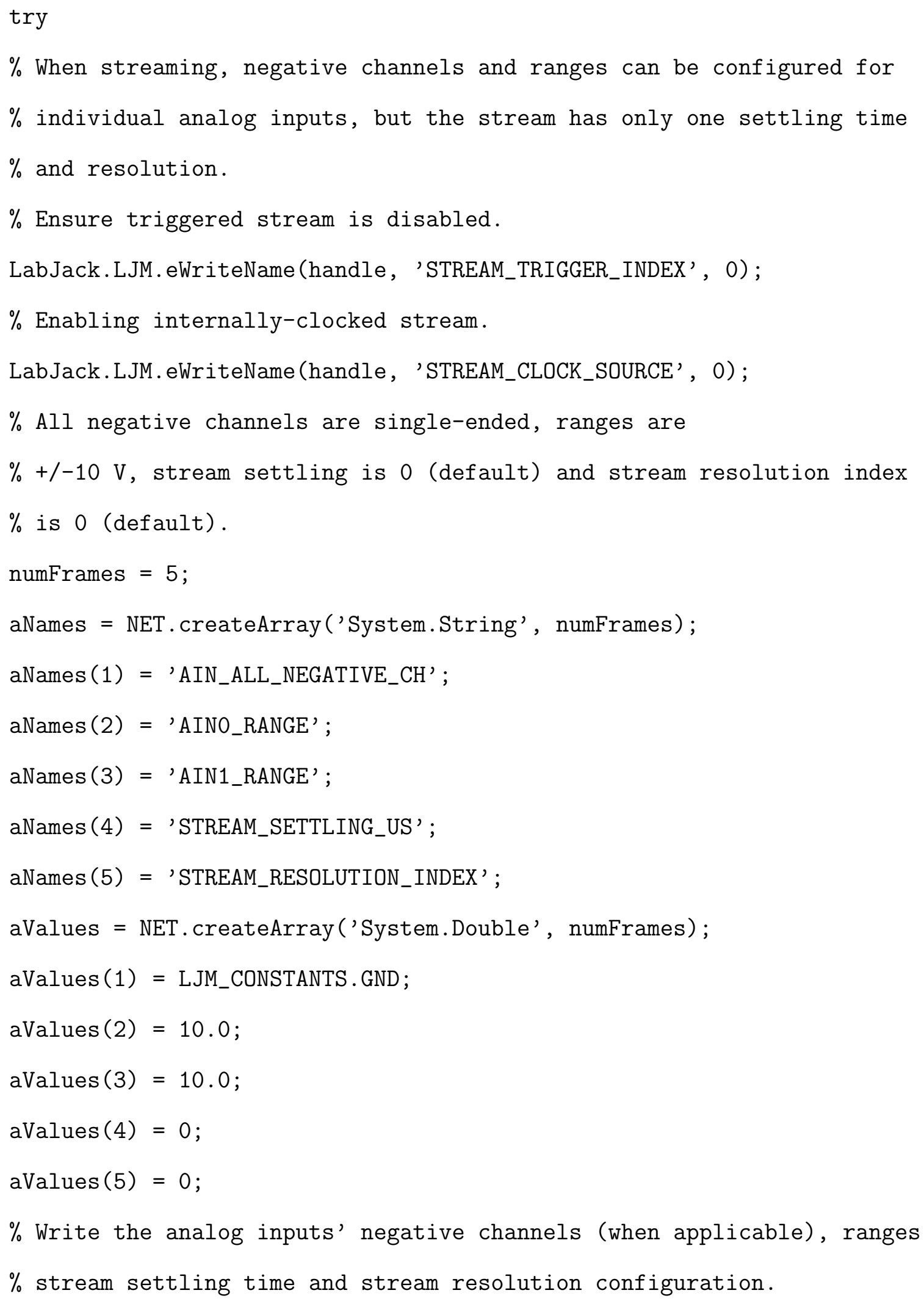


LabJack.LJM.eWriteNames (handle, numFrames, aNames, aValues, -1);

$\%$ Configure and start stream

numAddresses = aScanList. Length;

$[\sim$, scanRate $]=$ LabJack. LJM.eStreamStart (handle, scansPerRead, ..

numAddresses, aScanList, scanRate);

disp(['Stream started with a scan rate of ' num2str(scanRate)' Hz.'])

tic

disp(['Performing ' num2str(maxRequests)', stream reads.'])

totalScans $=0$;

curSkippedSamples $=0$;

totalSkippedSamples $=0$;

$\% \mathrm{k}=1$;

for $i=1:$ maxRequests

[ , devScanBL, ljmScanBL] = LabJack.LJM.eStreamRead(handle, aData, 0, 0);

totalScans = totalScans + scansPerRead;

\% Count the skipped samples which are indicated by -9999

$\%$ values. Skipped samples occur after a device's stream buffer

$\%$ overflows and are reported after auto-recover mode ends.

$\%$ When streaming at faster scan rates in MATLAB, try counting

$\%$ the skipped packets outside your eStreamRead loop if you are 
$\%$ getting skipped samples/scan.

curSkippedSamples $=\operatorname{sum}(\operatorname{double}($ aData $)==-9999.0)$;

totalSkippedSamples = totalSkippedSamples + curSkippedSamples;

mydata $(:, i)=\left(\operatorname{double}(\text { aData })^{\prime}\right)$;

$\%$ reshape data to be usable

mydatareshaped $=(\operatorname{reshape}(\operatorname{mydata}(:, i),[\text { numAINs, scansPerRead }]))^{\prime}$;

$\%$ voltageoutputs $(\mathrm{k}: \mathrm{k}+($ scansPerRead -1$), 1:$ numAINs $)=$ mydatareshaped

$\% \mathrm{k}=\mathrm{k}+\mathrm{s}$ cansPerRead;

end

timeElapsed = toc;

$\operatorname{disp}([$ 'Total scans $=$, num2str(totalScans)] $)$

$\operatorname{disp}([$ 'Skipped Scans $=$ ' num2str(totalSkippedSamples/numAddresses)])

$\operatorname{disp}([$ 'Time Taken $=$ ' num2str(timeElapsed) ', seconds'])

$\operatorname{disp}([$ LJM Scan Rate =' num2str(scanRate)', scans/second'])

$\operatorname{disp}([$ 'Timed Scan Rate = ' num2str(totalScans/timeElapsed) ', scans/second'])

$\operatorname{disp}([$ 'Sample Rate $=$ ' num2str (numAddresses*totalScans/timeElapsed) , ... samples/second'])

catch e

showErrorMessage (e)

end

disp ('Stop Stream')

LabJack.LJM. eStreamStop (handle);

\% Close handle

LabJack. LJM. Close (handle); 


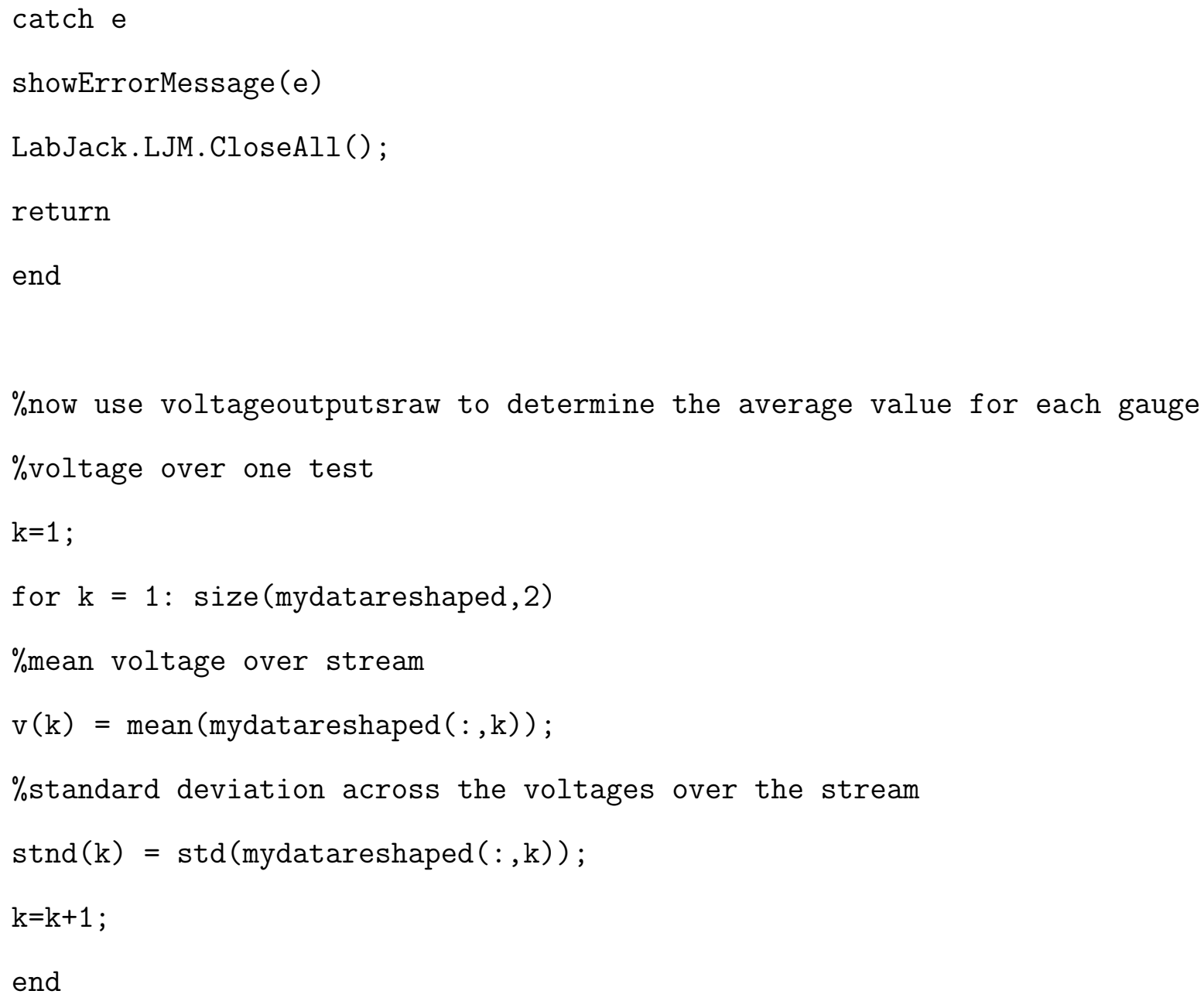




\section{Appendix I}

\section{Least Squares Approximation Matrices}

For any individual force input, lets say $F_{x}$, voltage outputs will be recorded. The relationship can be expressed as,

$$
F_{x}=b_{0}+b_{1} v_{1}+b_{2} v_{2}+b_{3} v_{3}+\ldots+b_{k} v_{k}
$$

where, $b_{0}$ represented the offset of the data, and $k$ represents the total number of voltage outputs. Arranging these in another way produces,

$$
F_{x}=\left[\begin{array}{llllll}
1 & v_{1} & v_{2} & v_{3} & \ldots & v_{k}
\end{array}\right]\left[\begin{array}{c}
b_{0} \\
b_{1} \\
b_{2} \\
b_{3} \\
\ldots \\
b_{k}
\end{array}\right]
$$


This process can be repeated for 6 desired input variables, and repeated for multiple trials across each of the 5 strain gauges. This would produce the relationship below, in which the highlighted section represented the extraction of 1-element from the input variable across all of the testing trials.

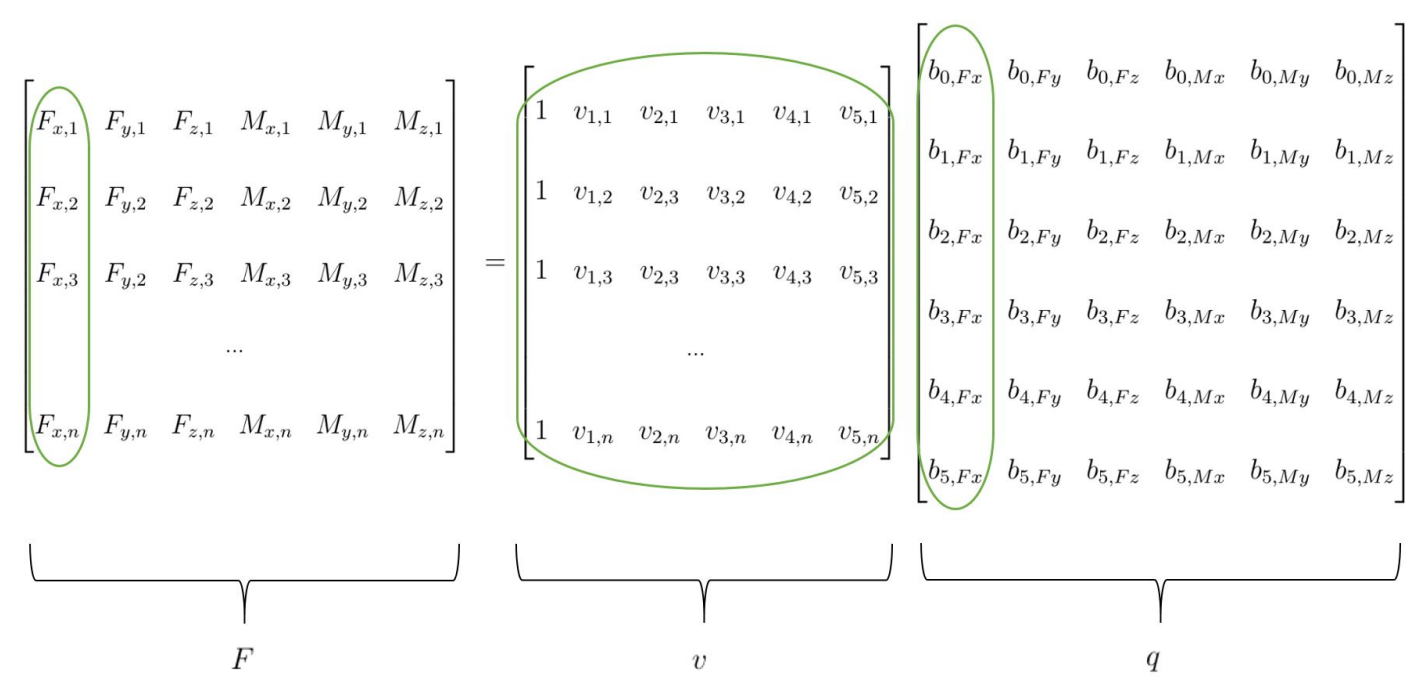

Therefore, this can be expressed algebraically as,

$$
\vec{v} q=\vec{F}
$$

rearranging,

$$
\hat{q}=\left(v^{T} v\right)^{-1} v^{T} \cdot \vec{F}
$$

where the first half of the $\hat{q}$ equation can be solved through pseudo-inverse. The estimation can then be returned into the system as,

$$
\vec{F}=\vec{v} \vec{q}
$$


I.1 Initial Validation of Least Square Approximation

Analysis of Combined Trials at a Single Point of Fz Load Application 

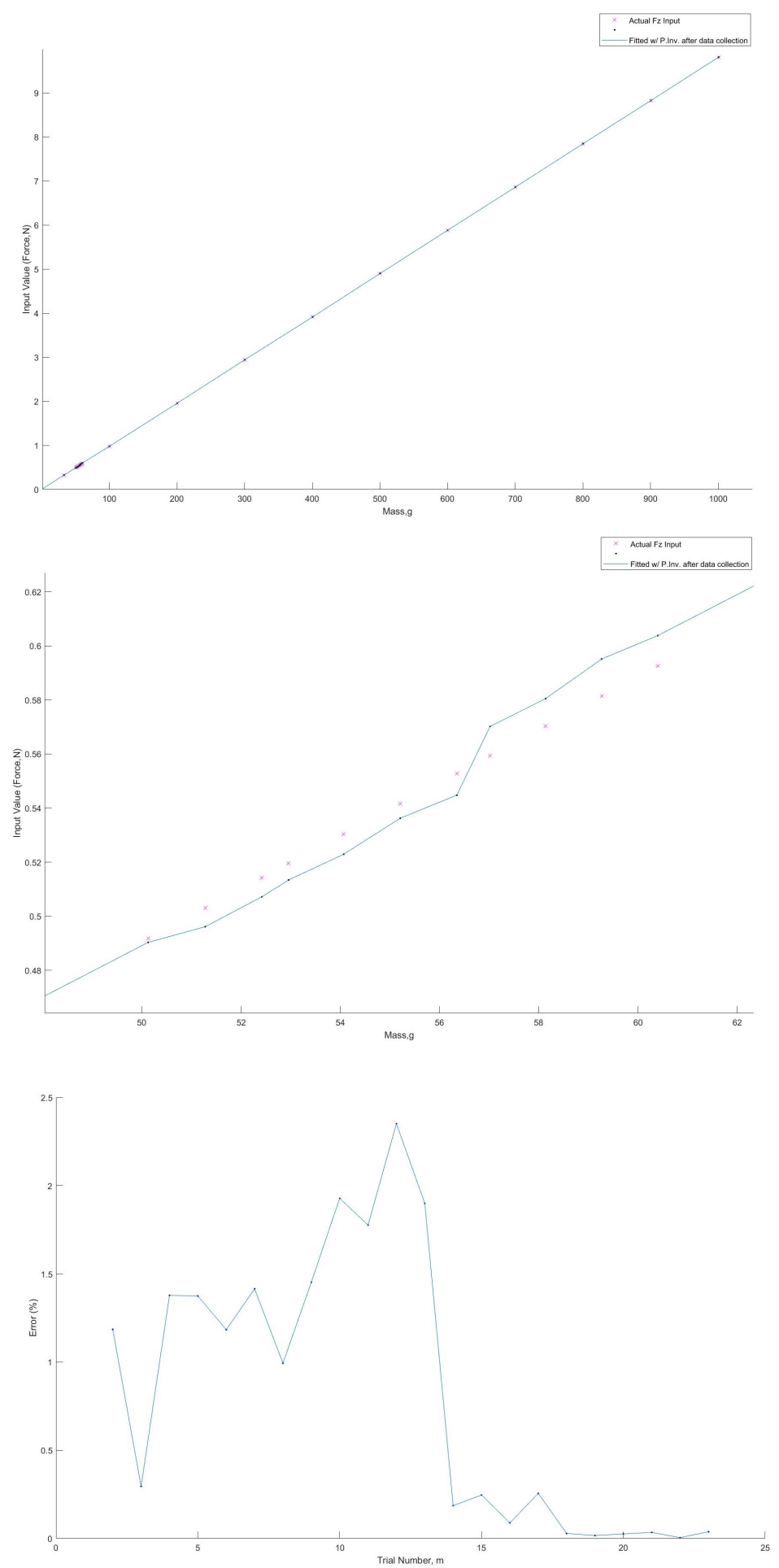

Figure I.1: Pseudo-Inverse Prediction Modelled Back to the Original Data at Position 2, with Focus on the Load Deviation in the $1 \mathrm{~g}$ Increments 

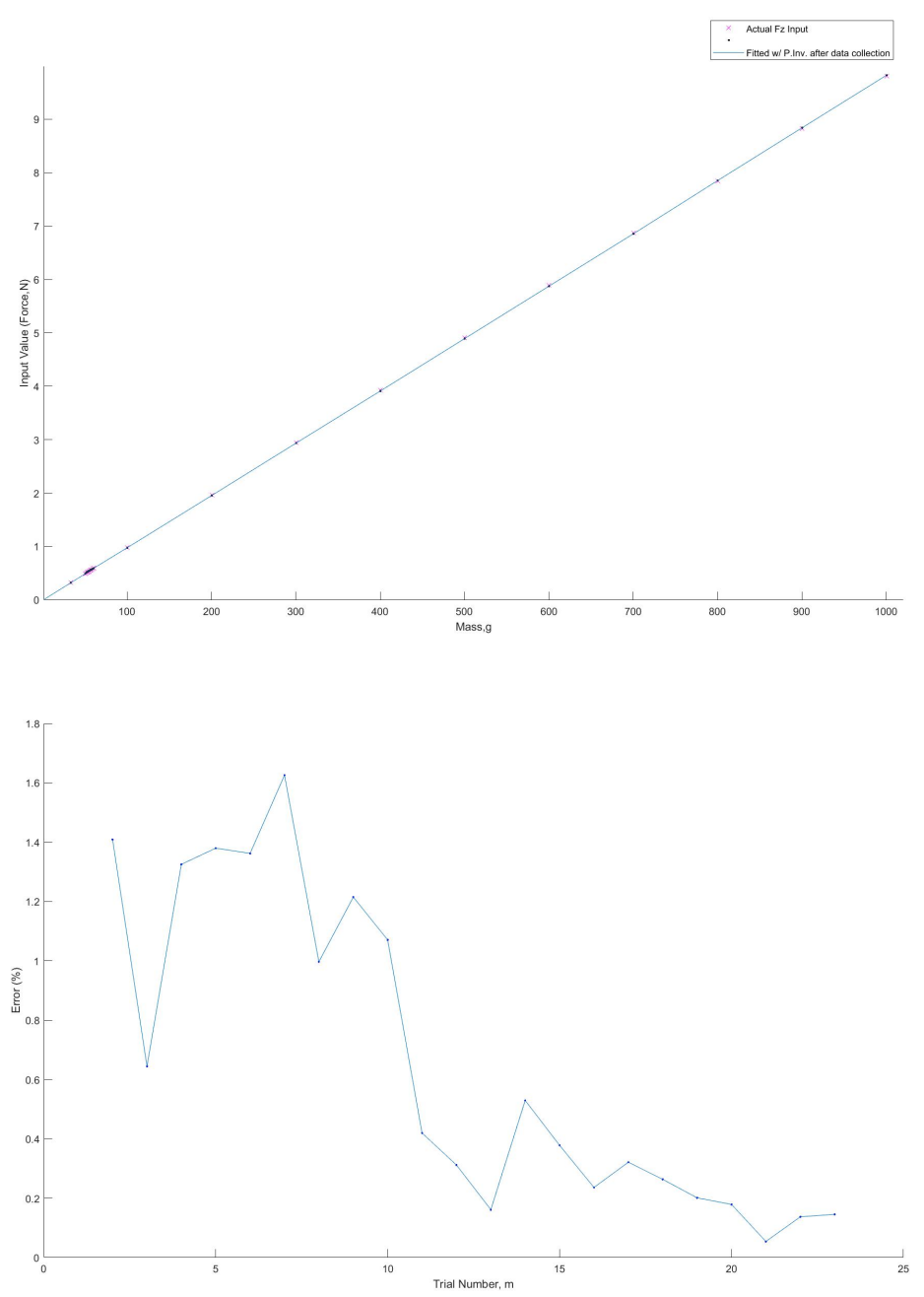

Figure I.2: Pseudo-Inverse Prediction Modelled Back to the Original Data at Position 3 
Analysis of Combined Trials at all Relevant Positions of Fz Load Application
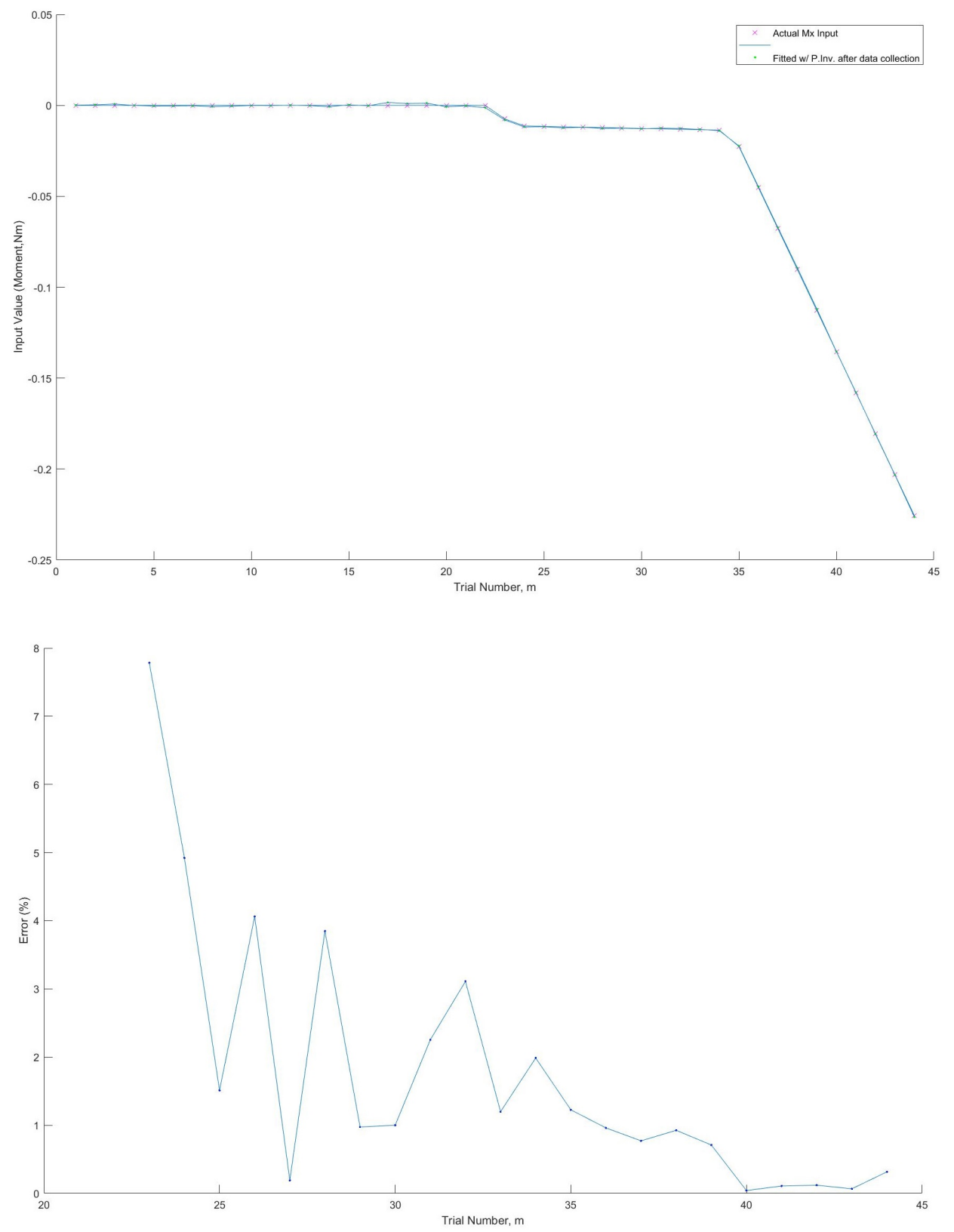

Figure I.3: Pseudo-Inverse Prediction of $M_{x}$ Modelled Back to the Original Data at Position 1 and Position 8 from $F_{z}$ Loading Tests Using the Voltage Outputs from All Gauge Pairs 

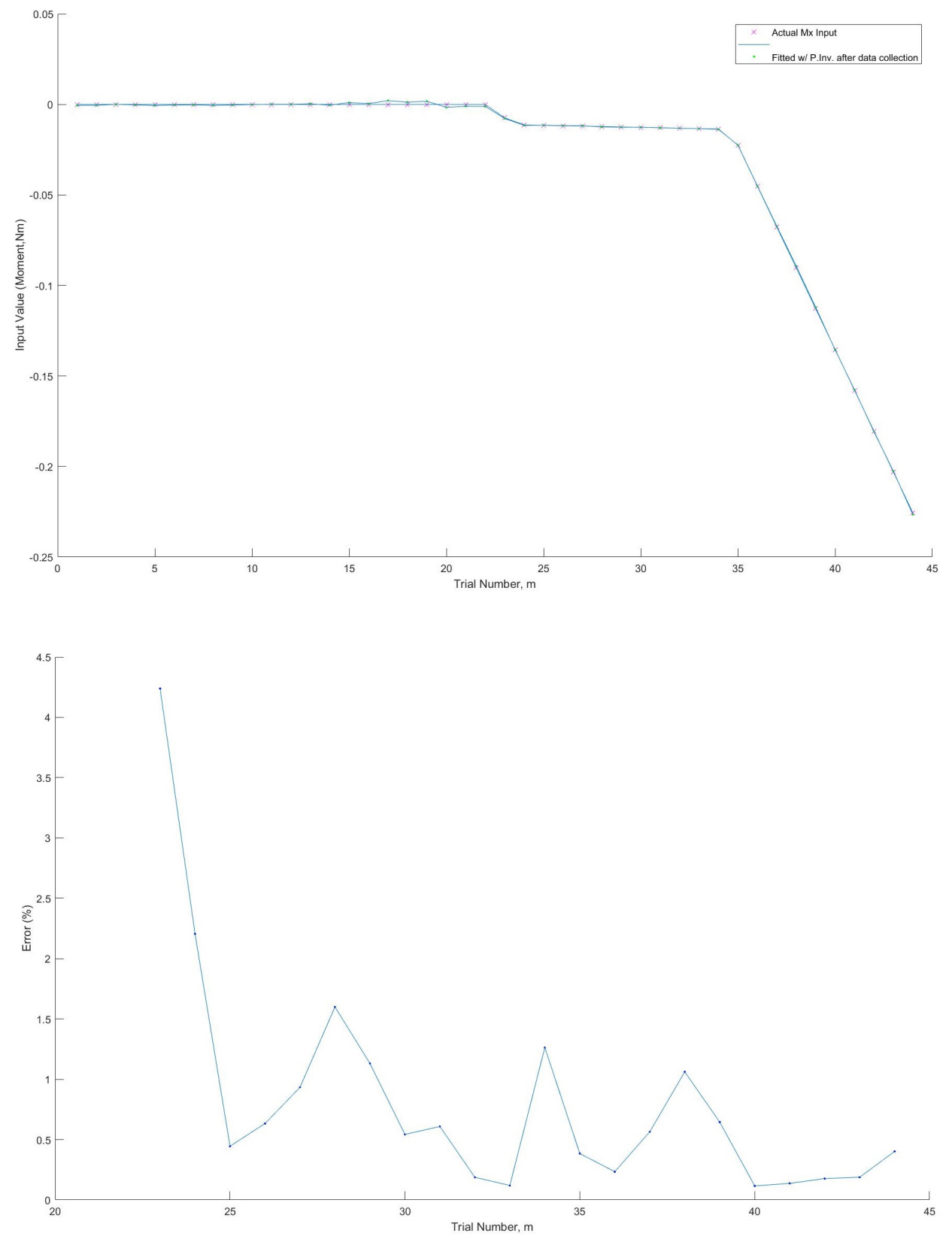

Figure I.4: Pseudo-Inverse Prediction of $M_{x}$ Modelled Back to the Original Data at Position 1 and Position 8 from $F_{z}$ Loading Tests Using the Voltage Outputs from the Linear Gauge Pairs 


\section{Appendix $\mathbf{J}$}

\section{Code Listing: Development of Calibration Matrix}

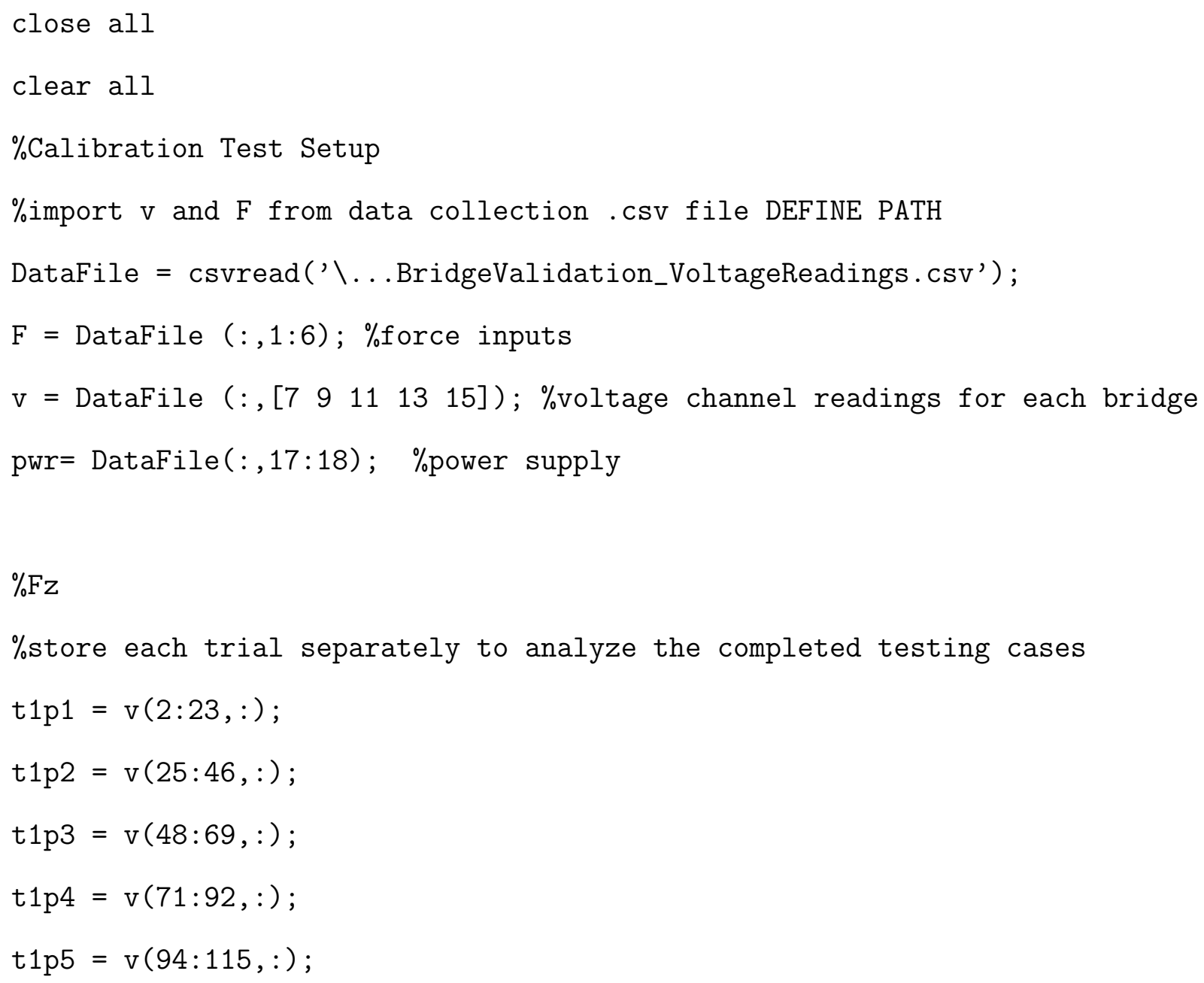




$$
\begin{aligned}
& \mathrm{t} 1 \mathrm{p} 6=\mathrm{v}(117: 138,:) ; \\
& \mathrm{t} 1 \mathrm{p} 7=\mathrm{v}(140: 161,:) ; \\
& \mathrm{t} 1 \mathrm{p} 8=\mathrm{v}(163: 184,:) ; \\
& \mathrm{t} 2 \mathrm{p} 1=\mathrm{v}(186: 207,:) \text {; } \\
& \mathrm{t} 2 \mathrm{p} 2=\mathrm{v}(209: 231,:) ; \\
& \mathrm{t} 2 \mathrm{p} 3=\mathrm{v}(233: 254,:) \text {; } \\
& \mathrm{t} 2 \mathrm{p} 4=\mathrm{v}(256: 277,:) \text {; } \\
& \mathrm{t} 2 \mathrm{p} 5=\mathrm{v}(279: 300,:) \text {; } \\
& \mathrm{t} 2 \mathrm{p} 6=\mathrm{v}(302: 323,:) ; \\
& \mathrm{t} 2 \mathrm{p} 7=\mathrm{v}(325: 346,:) ; \\
& \mathrm{t} 2 \mathrm{p} 8=\mathrm{v}(348: 369,:) ; \\
& \text { t3p1 = v }(372: 393,:) \text {; } \\
& \text { t3p2 = v }(395: 416,:) \text {; } \\
& \text { t3p3 = v }(418: 439,:) \text {; } \\
& \text { t3p4 = v }(441: 462,:) \text {; } \\
& \text { t3p5 = v }(464: 485,:) \text {; } \\
& \text { t3p6 = v }(487: 508,:) \text {; } \\
& \text { t3p7 = v }(510: 531,:) \text {; } \\
& \text { t3p8 = v }(533: 554,:) \text {; } \\
& \mathrm{t} 4 \mathrm{p} 1=\mathrm{v}(556: 577,:) ; \\
& \mathrm{t} 4 \mathrm{p} 2=\mathrm{v}(579: 600,:) ; \\
& \mathrm{t} 4 \mathrm{p} 3=\mathrm{v}(602: 623,:) \text {; } \\
& \mathrm{t} 4 \mathrm{p} 4=\mathrm{v}(625: 646,:) \text {; } \\
& \text { t4p5 = v }(648: 669,:) \text {; }
\end{aligned}
$$




$$
\begin{aligned}
& \mathrm{t} 4 \mathrm{p} 6=\mathrm{v}(671: 692,:) ; \\
& \mathrm{t} 4 \mathrm{p} 7=\mathrm{v}(694: 715,:) ; \\
& \mathrm{t} 4 \mathrm{p} 8=\mathrm{v}(717: 738,:) ;
\end{aligned}
$$

$\%$ now the repeated trials for the defined test positions are combined. This $\%$ is completed by finding the mean voltage at each loading case $i=1$;

$\mathrm{k}=1$;

Fz_p1 $=\operatorname{zeros}(\operatorname{size}(t 4 p 1))$;

Fz_p2 $=\operatorname{zeros}(\operatorname{size}(t 4 p 1)) ;$

Fz_p3 $=\operatorname{zeros}(\operatorname{size}(t 4 p 1))$;

Fz_p4 $=\operatorname{zeros}(\operatorname{size}(t 4 p 1))$;

Fz_p5 $=\operatorname{zeros}(\operatorname{size}(t 4 p 1))$;

Fz_p6 $=\operatorname{zeros}(\operatorname{size}(t 4 p 1)) ;$

Fz_p7 $=\operatorname{zeros}(\operatorname{size}(t 4 p 1))$;

Fz_p8 $=\operatorname{zeros}(\operatorname{size}(t 4 p 1))$;

for $i=1: 22$

Fz_p1 $(\mathrm{k},:)=\operatorname{mean}([\mathrm{t} 1 \mathrm{p} 1(\mathrm{i},:) ; \mathrm{t} 2 \mathrm{p} 1(\mathrm{i},:) ; \mathrm{t} 3 \mathrm{p} 1(\mathrm{i},:) ; \mathrm{t} 4 \mathrm{p} 1(\mathrm{i},:)]) ;$

Fz_p2 $(k,:)=\operatorname{mean}([\mathrm{t} 1 \mathrm{p} 2(i,:) ; \mathrm{t} 2 \mathrm{p} 2(\mathrm{i},:) ; \mathrm{t} 3 \mathrm{p} 2(i,:) ; \mathrm{t} 4 \mathrm{p} 2(i,:)]) ;$

Fz_p3 $(k,:)=\operatorname{mean}([\operatorname{t1p} 3(i,:) ; \operatorname{t2p} 3(i,:) ; \operatorname{t3p} 3(i,:) ; \operatorname{t4p} 3(i,:)])$;

Fz_p4 $(k,:)=\operatorname{mean}([\mathrm{t} 1 \mathrm{p} 4(i,:) ; \mathrm{t} 2 \mathrm{p} 4(\mathrm{i},:) ; \mathrm{t} 3 \mathrm{p} 4(\mathrm{i},:) ; \mathrm{t} 4 \mathrm{p} 4(\mathrm{i},:)]) ;$

Fz_p5 $(k,:)=\operatorname{mean}([\operatorname{t1p} 5(i,:) ; \operatorname{t2p} 5(i,:) ; \operatorname{t3p} 5(i,:) ; \operatorname{tpp} 5(i,:)]) ;$

Fz_p6 $(\mathrm{k},:)=\operatorname{mean}([\mathrm{t} 1 \mathrm{p} 6(\mathrm{i},:) ; \mathrm{t} 2 \mathrm{p} 6(\mathrm{i},:) ; \mathrm{t} 3 \mathrm{p} 6(\mathrm{i},:) ; \mathrm{t} 4 \mathrm{p} 6(\mathrm{i},:)]) ;$

Fz_p7 $(\mathrm{k},:)=\operatorname{mean}([\mathrm{t} 1 \mathrm{p} 7(\mathrm{i},:) ; \mathrm{t} 2 \mathrm{p} 7(\mathrm{i},:) ; \mathrm{t} 3 \mathrm{p} 7(\mathrm{i},:) ; \mathrm{t} 4 \mathrm{p} 7(\mathrm{i},:)]) ;$

$\mathrm{Fz}_{-} \mathrm{p} 8(\mathrm{k},:)=\operatorname{mean}([\mathrm{t} 1 \mathrm{p} 8(\mathrm{i},:) ; \mathrm{t} 2 \mathrm{p} 8(\mathrm{i},:) ; \mathrm{t} 3 \mathrm{p} 8(i,:) ; \mathrm{t} 4 \mathrm{p} 8(\mathrm{i},:)]) ;$

$i=i+1$;

$\mathrm{k}=\mathrm{k}+1$; 
end

$\%$ saving the input conditions at each testing position

Fz_p1f $=F(2: 23,:)$;

Fz_p2f $=F(25: 46,:)$;

Fz_p3f $=F(48: 69,:)$;

Fz_p4f $=F(71: 92,:)$;

Fz_p5f $=F(94: 115,:)$;

$F z_{-}$p6f $=F(117: 138,:)$;

$F z_{-} p 7 f=F(140: 161,:)$;

Fz_p8f $=F(163: 184,:)$;

$\%$ this process would be repeated for Fx and Fy tests

$\%$ now combine to provide a snapshot of all the relevant experiemntal trials $\%$ for the force application

$\%$ select the columns of $\mathrm{v}$ to consider (1- linear, 2-liner, 3-45, 4-45, 5-0) $\mathrm{v}_{-} \mathrm{Fzcal}=\left[\mathrm{Fz} z_{-} \mathrm{p} 1\left(:,\left[\begin{array}{ll}1 & 2\end{array}\right]\right) ; \mathrm{Fz} z_{-} \mathrm{p} 2\left(:,\left[\begin{array}{ll}1 & 2\end{array}\right]\right) ; \mathrm{Fz} \_\mathrm{p} 3\left(:,\left[\begin{array}{ll}1 & 2\end{array}\right]\right) ; \mathrm{Fz} z_{-} \mathrm{p} 4\left(:,\left[\begin{array}{ll}1 & 2\end{array}\right]\right) ; \ldots\right.$

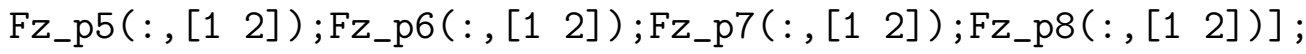
$F_{-} F_{z c a l}=\left[F_{-}\right.$p1f $\left.; F z_{-} p 2 f ; F z_{-} p 3 f ; F z_{-} p 4 f ; F z_{-} p 5 f ; F z_{-} p 6 f ; F z_{-} p 7 f ; F z_{-} p 8 f\right] ;$

\section{$\% \% \% \% \% \% \% \% \% \%$}

$\%$ select which to calibrate for

$\mathrm{v}=\mathrm{v}_{-}$Fzcal

$F=F \_F z c a l$

$\% \% \% \% \% \% \% \% \% \% \%$ 
$\%$ add ones column, to account for the initial offset in the $q$ matrix $c 1=\operatorname{ones}($ length $(v), 1) ;$

\%development of voltage matrix in least squares approximation form $\mathrm{v}=\left[\begin{array}{cc}\mathrm{c} 1 & \mathrm{v}\end{array}\right]$;

v_size $=\operatorname{size}(v)$;

\%complete pseudo-inverse calculation for each column of $\mathrm{F}$ $\mathrm{q}=\mathrm{v} \backslash \mathrm{F}$

$\%$ where, $q=$ [q_pinv_Fx, q_pinv_Fy, q_pinv_Fz, q_pinv_Mx, q_pinv_My, q_pinv_Mz]; $\%$ save $q$ values to use in Input Calculation Script to be applied to final \%LacJack Data Collection Script

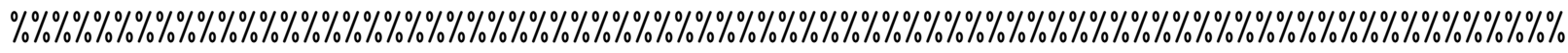

$\%$ test inverse calibration result

\%the following plots allow visual assessment of the accuracy in the psinv $\%$ prediction

$\%$ first they are applied back to their original data $\%$ these are the estimated inputs from using the calibration method $F_{-}$pinv $=v * q$

$\%$ these are the percent error of reaching true inputs percenterror_Fx $=\operatorname{abs}\left(\left(\left(F_{-} p i n v(:, 1)-F(:, 1)\right) . / F(:, 1)\right) * 100\right)$; percenterror_Fy $=\operatorname{abs}\left(\left(\left(F_{-}\right.\right.\right.$pinv $\left.\left.\left.(:, 2)-F(:, 2)\right) . / F(:, 2)\right) * 100\right)$; percenterror_Fz $=\operatorname{abs}\left(\left(\left(F_{-}\right.\right.\right.$pinv $\left.\left.\left.(:, 3)-F(:, 3)\right) . / F(:, 3)\right) * 100\right)$; percenterror_Mx $=\operatorname{abs}\left(\left(\left(F_{-}\right.\right.\right.$pinv $\left.\left.\left.(:, 4)-F(:, 4)\right) . / F(:, 4)\right) * 100\right)$; percenterror_My $=\operatorname{abs}\left(\left(\left(F_{-} \operatorname{pinv}(:, 5)-F(:, 5)\right) . / F(:, 5)\right) * 100\right)$; percenterror_Mz $=\operatorname{abs}\left(\left(\left(F_{-} \operatorname{pinv}(:, 6)-F(:, 6)\right) . / F(:, 6)\right) * 100\right)$; 


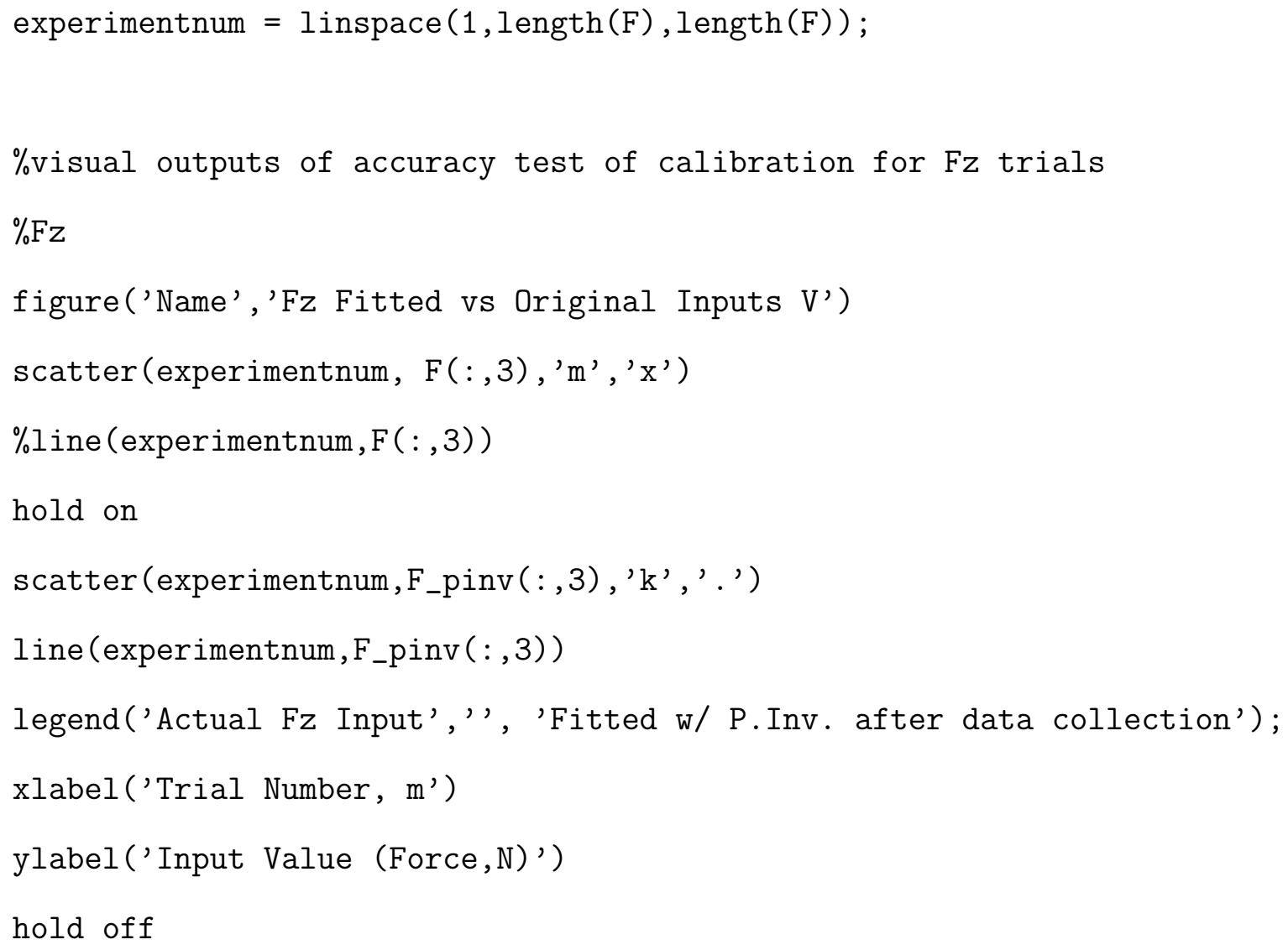




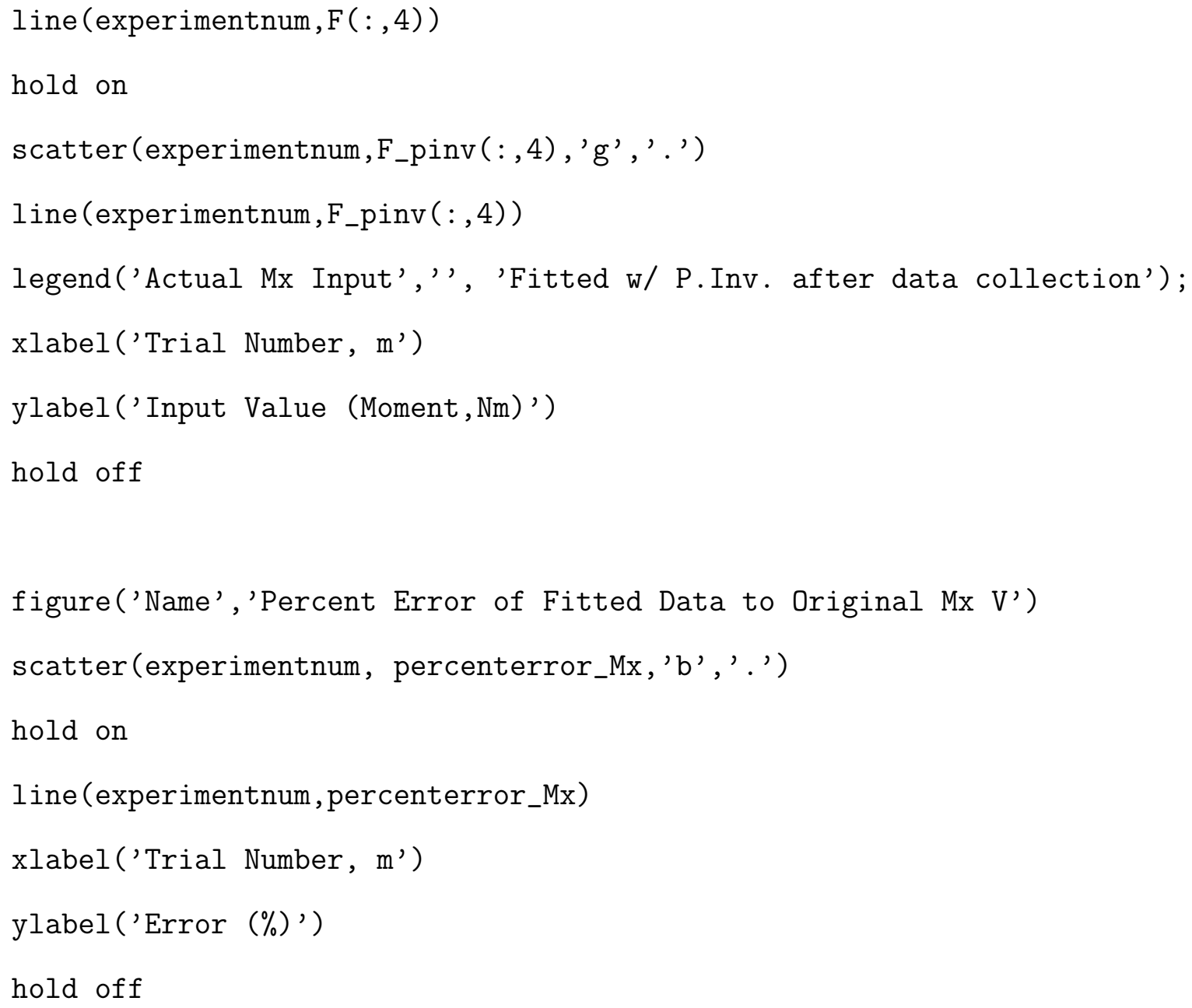

